\title{
Filogenia das espécies deLutzomyia França, 1924 (Diptera, Psychodidae, Phlebotominae)
}

\section{Rute Maria Gonçalves de Andrade}

Tese apresentada ao Programa de Pósgraduação em Saúde Pública para da Faculdade de Saúde Pública da Universidade de São Paulo para obtenção do título de Doutor em Saúde Pública

Área de Concentração: Epidemiologia Orientador: Profa. Dra. Eunice Aparecida Bianchi Galati

\section{São Paulo}




\section{4 doe}

Autorizo. exclusivamente para fins acadêmicos e científicos. a reprodução total ou parcial desta tese. por processos fotocopiadores.

Assinatura

Data 


\section{DEDICATÓRIA}

Aos meus pais, por tudo.

Aos meus queridos Francisco e Rodrigo

pelo amor, paciência, compreensão, estímulo, e carinho. 


\section{AGRADECIMENTOS ESPECIAIS}

Á amiga Renilda Maria de Figueiredo Shimono (Secretaria de Pós-graduação, FSP USP) e ao Professor Sergio Antonio Vanin (Instituto de Biociências - USP): sem o carinho e o profissionalismo de ambos eu não teria chegado ao final! 


\section{AGRADECIMENTOS TAMBÉM ESPECIAIS}

Á Professora Eunice Aparecida Bianchi Galati, pela orientação, carinho e disponibilidade sempre constantes.

Aos Professores Sergio Antonio Vanin (Instituto de Biociências -USP), Almério de Castro Gomes (Departamento de Epidemiologia, FSP-USP), Délsio Natal (Departamento de Epidemiologia, FSP-USP), Antônio Carlos Marques (Instituto de Biociências - USP) e Júlio da Mota Singer (Instistuto de Matemática e Estatísitca - USP) pelo carinho, paciência, estímulo e assessoria.

Ás secretárias da Pós-graduação e amigas Renilda Maria de Figueiredo Shimono, Maria Aparecida Mendes e Márcia Aparecida Garcia da Silva pela atenção e amizade sempre presentes

Ao Professor Oswaldo Paulo Forattini pela atenção e pelo empréstimo das làminas da coleção da Faculdade de Saúde Pública-USP.

A Alda Lima Falcão e José Dilermando Andrade Filho pelo empréstimo das làminas e auxílio com o dados das espécies do Museu do Instituto René Rachou, Minas Gerais.

A Mirna Ferracini pela disponibilidade e profissionalismo sempre presentes

A Denise Vilarinho Tambourgi e Patricia Vilarinho Tambourgi pelo carinho, paciência, amizade, confiança e ajuda sempre presente.

Ao Lauro Kazumi Dehira, Wilma e Wilson do IPT-USP pela disponibilidade, profissionalismo e carinho com que prestaram auxilio.

Ao Ademir,ao Rafael e ao Fernado Galati pela amizade e colaboração.

A Paloma Helena Fernandes Shimabukuro pelo carinho, disponibilidade e ajuda.

Aos amigos e profissionais do Laboratório de Imunoquimica: José Cassiano Borba. Fábio Carlos Magnoli, Jorge Mário Ferreira Júnior, Elaine Rodrigues, Silvia Aparecida Camargo, José Severino Ramos, Aparecida Santo Pietro, Maura Coração, João Batista Coelho, Matheus Ferracini, Danielle Paixão Cavalcante, Fernando Delgado Pretel, Matheus Fernades Pedrosa, Sônia Andrade pela atenção, carinho, paciência, confiança e auxilio

A amiga Ana Maria Marassá pelo carinho e amizade sempre presente.

Aos amigos Marcia Bicudo e Mauro Breviglieri Fonseca, pela amizade, estimulo e carinho constantes 
Aos amigos Bárbara Moisés Pain e Luis Antônio Pain pela disponibilidade e ajuda. Aos amigos e profissionais da Faculdade de Saúde Pública Odete, Janete, Edna, Aristides, Paulo, Walter, Rose, Marcia Gutierrez, Dulce, Ana Maria, Izilda pela amizade, estimulo e carinho 


\section{RESUMO}

Gonçalves-de-Andrade RM. Filogenia das espécies de Lutzomyia França, 1924 (Diptera, Psychodidae, Phlebotominae) São Paulo; (BR):2004. [Tese de Doutorado-Faculdade de Saúde Pública da USP]

Flebotomineos são insetos da ordem Diptera, familia Psychodidade, subfamilia Phlebotominae, cujo hábito hematofágico das fêmeas determina a importância das espécies para a saúde pública, uma vez que muitas são reconhecidas como vetores de leishmanioses e bartonelose, e como hospedeiras de diversos microorganismos incluindo arbovirus. Até o momento, mais de 800 espécies foram descritas, 464 delas endêmicas da região Neotropical e cerca de 40 identificadas como capazes de transmitir Leishmania Ross, 1903 (Kinetoplastida, Trypanosomatidae). Frente a essa diversidade, ampla distribuição geográfica e importância, classificações para o grupo, baseadas principalmente em caracteres morfológicos internos e externos, vêm sendo propostas por diversos autores que têm divergido quanto a considerar um menor ou maior número de gêneros ou categorias supra e subgnéricas. Na maioria dos sistemas de classificação o gênero Lutzomyia França, 1924 é considerado o mais importante da América, pois embora sua composição de espécies seja variável em cada uma delas, sempre estão presentes, nesse táxon, vetores de leishmaniose visceral e tegumentar. Assim sendo, foi feita análise cladística de 63 espécies de Lutzomyia com matriz composta por 63 caracteres polarizados segundo o método de comparação com o grupo externo. Como grupo externo utilizaram-se 19 táxons de outros gêneros de Phlebotominae e de Bruchomyiinae. O cladograma obtido revelou que Lutzomyia é monofilético e que contém apenas dois subgrupos monofiléticos considerados como os subgêneros Lutzomyia s. str. e Helcocyrtomyia. Uma classificação para o gênero é proposta. A topologia do cladograma é mostrada em notação parentética: (( $L$. ischnacantha (L. bifoliata (( L. souzalopesi (L. gaminarai ((L. alencari (L. cruzi. L. ischyracantha)) (L. longipalpis L. pseudolongipalpis)))) (L. dispar (L. lichyi ((L. castroi (L. amarali L. caligata)) (L. falcata (L. cruciata (L. gomezi ((L. diabolica (L. marinkellei (L. evangelistai (L. carvalhoi (L. spathotrichia (L. araracuarensis L. legerae)))))) (L. flabellata L. sherlocki))))))))))) (L. bicormuta (L. battistinii (L. forattinii (L. almerioi (L. cavernicola L. renei $)))))((L$. pallidithorax $((L$. pescei $(L$. osornoi (L. tejadai (L. galatiae. $(L$. 
quillabamba (( $L$. caballeroi. ( $L$. castanea (L. larensis, ( $L$. ceferinoi, $L$. herreri), $(L$. munaypata L. rispaili))))) (L. strictivilla, $(L$. wattsi ( $L$. gonzaloi ( $L$. hartmanni ( $L$. kirigetiensis ( $L$. sanguinaria $(L . \mathrm{sp}$ de Pichinde ( $L$. cirrita ( $L$. scorzai, $(L$. tortura $(L$. guderiani (L. adamsi,L. monzonensis))))))))))))))))) (L. ayacuchensis L. peruensis))) (L. blancasi (L. chavinensis L. noguchii)).

Descritores: Phlebotominae. Lutzomyia $\quad s . \quad$ str. Leishmaniose. Sistemática Filogenética 


\section{SUMMARY}

Gonçalves-de-Andrade RM. Phylogenetic analysis of Lutzomyia França, 1924 genus (Diptera, Psychodidae, Phlebotominae) São Paulo; (BR):2004. [PhD Thesis-Faculdade de Saúde Pública da USP]

Phlebotomines are insects from the Diptera order, Psychodidade family, Phlebotominae subfamily, whose haematophagous habit of the females determines the species importance for public health, once many are recognized both as leishmaniasis and bartonelose vectors, and also as hostesses for several microorganisms, including arbovirus. So far, more than 800 species have been described, 464 of wich are endemic in the Neotropical region and about 40, identified as transmiters of Leishmania Ross, 1903 (Kinetoplastida, Trypanosomatidae). Considering such diversity, wide geographical distribution and importance, classifications have been proposed by several authors, based on internal and external morphological characters. These authors have diverged about the number of genders informal categories. In the majority of the classification systems, Lutzomyia França, 1924 is considered the most important in America, because, although the species composition is variable, visceral and tegumentar leishmaniasis vectors are always present in this taxon. Thus, a cladistic analysis of 63 Lutzomyia's species was performed, using 63 polarized characters. As outgroup, it has been used 19 species of other Phlebotominae's genus and Bruchomyiinae subfamily. The analysis of the cladogram has shown that Lutzomyia is monophyletic and contains two monophyletics subgroups, which can be considered as the subgenus Lutzomyia s. str. and Helcocyrtomyia. A classification to Lutzomyia genus is proposed. The cladogram topology is presented in parenthetic notation: (((L. ischnacantha (L. bifoliata ((L. souzalopesi (L. gaminarai ((L. alencari (L. cruzi. L. ischyracantha)) (L. longipalpis L. pseudolongipalpis)))) (L. dispar (L. lichyi ((L. castroi (L. amarali L. caligata)) (L. falcata (L. cruciata (L. gomezi ((L. diabolica (L. marinkellei ( $L$. evangelistai (L. carvalhoi (L. spathotrichia (L. araracuarensis L. legerae)))))) (L. flabellata L. sherlocki))))))))))) (L. bicormuta (L. battistinii (L. forattinii (L. almerioi (L. cavernicola L. renei $)))))((L$. pallidithorax $((L$. pescei $(L$. osornoi $(L$. tejadai (L. galatiae. $(L$. quillabamba ((L. caballeroi. ( $L$. castanea (L. larensis, ( $L$. ceferinoi,L. herreri), $(L$. 
munaypata L. rispaili))))) (L. strictivilla, $(L$. wattsi $(L$. gonzaloi (L. hartmanni ( $L$. kirigetiensis ( $L$. sanguinaria $(L . \mathrm{sp}$ de Pichinde $(L$. cirrita $(L$. scorzai, $(L$. tortura $(L$. guderiani (L. adamsi,L. monzonensis))))))))))))))))) (L. ayacuchensis L. peruensis))) $(L$. blancasi (L. chavinensis L. noguchii)).

Descriptors: Phlebotominae. Lutzomyia $s . \quad$ str. Leishmaniasis. Phylogenetic Systematic. 
INDICE 


\section{ÍNDICE}

l INTRODUÇÃO 1

1.1 Histórico 3

2 OBJETIVOS 10

2.1 Objetivo Geral 10

$\begin{array}{ll}2.2 \text { Objetivos específicos } & 10\end{array}$

3 JUSTIFICATIVA 11

4 MATERIAL 13

4.1 Obtenção e preparo do material utilizado 13

4.2 Métodos 14

4.2.1 Morfometria $\quad 15$

4.2.2 Metodologia de análise dos dados morfométricos 15

4.2.3 Análise cladística 16

$\begin{array}{ll}4.3 \text { Ilustração } & 17\end{array}$

5 RESULTADOS E DISCUSSÃO 18

5.1 Análise dos caracteres 18

5.2 Análise do cladograma $\quad 54$

5.3 Discussão geral 57

5.4 Classificação de Lutzomyia $\quad 60$

6 CONCLUSÕES

7 REFERÊNCIAS BIBLIOGRÁFICAS 65

ANEXOS

Material examinado e procedência Anexos A-1

Lista de espécies estudadas para análise cladística de Lutzomyia Anexos A-12

Glossário Anexos A-15

Lista de caracteres Anexos A-20

Tabelas Anexos A-25

Matriz Anexos A-43

Figuras - Gráficos Anexos A-45

Cladogramas Anexos A-63

$\begin{array}{ll}\text { Ilustrações } & \text { Anexos A-67 }\end{array}$ 
INTRODUÇÃO 


\section{Introdução}

As Leishmanioses acometem populações humanas em vinte e dois paises do Novo Mundo e em sessenta e seis do Velho Mundo, onde são endèmicas. Estima-se que anualmente cerca de 1,5 milhão de pessoas são infectadas pelas formas tegumentares e aproximadamente 500 mil pela forma visceral. Cerca de $90 \%$ dos casos da forma cutànea são registrados no Irã, Afeganistão, Nepal, Síria. Arábia Saudita, Brasil e Peru e mais de $90 \%$ dos acometidos pela forma visceral estão em Blangadesh, Brasil, Índia, Nepal e Sudão (WHO 2002)

A forma cutànea da doença não é fatal e é a mais comum, podendo em alguns casos causar lesões desfigurantes. A forma visceral pode ser fatal e tem causado milhares de mortes a cada ano (WHO 2002).

A transmissão desta zoonose se dá pela picada de fêmeas de flebotomineos.

Flebotomíneos são insetos da ordem Diptera, familia Psychodidade, subfamilia Phlebotominae, cujo hábito hematofágico das fêmeas determina a importância das espécies para a saúde pública, uma vez que muitas delas são reconhecidas como vetores das já referidas leishmanioses, da bartonelose (YOUNG e DUNCAN 1994), e também como hospedeiras de diversos microorganismos, incluindo arbovírus (SHAW e col. 2003).

Até o momento, mais de 800 espécies foram descritas, 464 delas endêmicas da região Neotropical (YOUNG e DUNCAN 1994; GALATI 2003) e cerca de 40 identificadas como capazes de transmitir Leishmania Ross, 1903 (Kinetoplastida, Trypanosomatidae) (PIMENTA e col. 2003) 
Frente a essa importància. diversidade e ampla distribuição geográfica, classificaçōes para o grupo. baseadas principalmente em caracteres morfológicos internos e externos. vèm sendo propostas por diversos autores que. de maneira geral, têm divergido quanto a se considerar um menor ou maior número de gèneros ou categorias supra e subgenéricas (FRANÇA 1921. DYAR 1929, MANGABEIRA 1941. THEODOR 1948. 1965, FAIRCHILD 1955. BARRETTO 1962, FORATTINI 1971. YOUNG e FAIRCHILD 1974, ABBONENC e LEGER 1976, LEWIS e col. 1977, MARTINS e col. 1978, VARGAS 1978, ARTEMIEV 1991, YOUNG e DUNCAN 1994, GALATI 1995).

Estas classificações. com exceção da de GALATI 1995. proposta com base na metodologia filogenética de HENNIG 1966, em sua maioria, fundamentam-se em semelhanças morfológicas observadas, sendo bastante variável o elenco de caracteres analisados pelos diveros autores. Mais recentemente, essa metodologia tem sido aplicada por outros autores que tèm elaborado propostas de filogenia entre grupos de flebotomineos com base, especialmente, em marcadores moleculares, porém estas abordagens ainda são poucas e as controversias permanecem (ARANSAY e col. 2000, MAZZONI e col 2002, TORGERSON e col. 2003, BEATI e col. 2004).

Na maioria dos sistemas de classificação o gênero Lutzomyia França, 1924 é considerado o mais importante da América, pois embora sua composição de espécies seja variável. em cada uma delas estão presentes, nesse táxon, vetores de leishmaniose visceral e tegumentar. Assim sendo, a analise cladística das espécies desse gênero, escopo deste trabalho, poderá fornecer ferramentas para reconstrução filogenética dos flebotomineos americanos. 


\subsection{HISTÓRICO}

A familia Psychodidae, a mais basal dos Psychodomorpha (Diptera) (WOOD e BORKENT 1989) tem sido aceita com cinco subfamílias: Bruchomyiinae. Psychodinae, Phlebotominae, Sycoracinae e Trichomyiinae (HENNIG 1972).

Devido à grande diversidade de espécies de Phlebotominae e sua importância em saúde. várias têm sido as propostas taxonômicas para o grupo. Ao longo da história controvérsias tem havido a respeito de se considerar um número maior de gèneros ou de categorias supra e subgenéricas. Este breve histórico enfocará principalmente as classificações que trataram de espécies da América e do gênero Lutzomyia França, 1924, objeto deste estudo.

Lutzomyia foi criado por FRANÇA em 1924 como subgênero para a espécie americana Phlebotomus longipalpis Lutz \& Neiva, 1912, em substituição a Lutzia, nome criado por Theobald, 1903 para um grupo de Culicidae (Diptera). Anteriormente, o gênero Phlebolomus Rondani, 1840 já havia sido dividido por FRANÇA (1919) em dois subgèneros, Phlebotomus s. str. e Newsteadia França, 1919.

FRANÇA e PARROT (1920) propuseram a substituição de Newsteadia por Sergentomyia pelo fato de ter sido criado por Green, 1902 como gênero de Ortheziidae (Hemoptera, Coccoidea) e descreveram o subgênero Neophlebotomus. Em 1921, esses mesmos autores propuseram um outro sistema no qual adotaram para Phlebotomus os três subgêneros anteriores: Phlebotomus $s$. str., Sergentomyia e Neophlebotomus e 
criaram outros dois: Brumptomyia e Prophlebotomus. Este último foi considerado por COSTA LIMA (1932) como sinonnimo de Sergentomyia

DYAR (1929) criou o subgênero Shannonomyia e adotou outros très para Phlebotomus: Brumptomyia França \& Parrot, 1921, Neophlebotomus França e Parrot, 1921 e L.utzomyia França, 1924.

COSTA LIMA (1932) revisou o conhecimento sobre os flebotomos americanos e inseriu todas as especies descritas no gènero Phlebotomus, para o qual considerou os subgèneros Sergentomyia, Brumptomyia, Neophlebotomus, Lutzomyia e criou Pintomvia

MANGABEIRA (1941) adotou para Phlebotomus os subgèneros: Lutzomyia, Brumptomyia e Shannonomyia; acrescentou Pintomyia; descreveu Evandromyia, Psychodopygus, Viannamyia e, em 1942, descreveu os subgèneros Pressatia e ('astromyia Nesta classificação o subgênero Sergentomyia não foi considerado.

ADDIS (1945) criou Dampfomyia como subgênero novo para um grupo de flebotomineos da América

Em 1948. THEODOR elevou os subgèneros Phlebotomus, Sergentomyia Brumptomyia e Lutzomyia para a categoria de gènero. Nos dois primeiros inseriu espécies do Velho Mundo e nos dois últimos, espécies da América.

Neste mesmo ano, dois novos gêneros para as espécies americanas foram descritos Warileya por HERTIG e Hertigia por FAIRCHILD

FAIRCHILD (1955) propôs uma classificação para a familia Psychodidae na qual adotou para Phlebotominae duas tribos: Bruchomyiini e Phlebotomini. Esta última tribo com três gèneros Phlebotomus, Warileya e Hertigia. Phlebotomus foi dividido em 
cinco subgèneros: Phlebotomus s. str., Sergentomyia, Psychodopygus, liammamyia e Brumptomyia: os dois primeiros para espécies do Velho Mundo e os très últimos para as Neotropicais. Dividiu ainda os subgèneros, com exceção de Viamnamyia, em grupos de espécies

BARRETTO (1962) considerou Lutzomyia, Brumptomyia e Warileya como gèneros para os flebotomineos americanos. Para o gènero Lutzomyia reconheceu sete subgèneros já descritos (Lutzomyia s. str, Pintomyia, Evandromyia, Psychodopygus, Viannamyia, Pressatia e Dampfomyia) e propôs outros oito: Micropygomia, Sciopemyia, Helcocyrtomyia, Trichophoromyia, Coromyia, Trychopygomyia, Nyssomyia e Psathyromyza.

Em 1965, THEODOR propôs nova classificação na qual adotou quatro gêneros: Hertigia, Warileya, Brumptomyia e Lutzomyia. Para Lutzomvia considerou oito dos quinze subgèneros de BARRETTO (1962): Lutzomyia $s$. str. com duas séries de espécies, Psychodopygus com três séries de espécies, Evandromyia, l'iannamyia, Castromyia, Pressatia, Pyntomyia e Dampfomyia. Além desses subgèneros criou dezesseis grupos de especies subdivididos em séries de espécies

Em 1965, MARTINS e SILVA descrevaram Barrettomyia como subgênero de Lutzomyia

FORATTINI (1971) propôs uma classificação para Phlebotominae baseando-se em um elenco maior de caracteres morfológicos, incluindo alguns de formas imaturas. Nesta classificação adotou sete gêneros: Warileya, Brumptomyia, Lutzomyia, Psychodopygus, Pintomyia, Pressatia e Viannamyia. Dividiu o gènero Lutzomyia em seis subgèneros: Lutzomyia s. str., Dampfomyia, Micropygomyia, Coromyia, 
Irichopygomyia e Barrettomyia. O gènero Hertigia foi alocado em Bruchomyiinae (Psychodidae).

LEWIS e col. (1977) propuseram cinco gêneros para os Phlebotominae: Warileya. Phlebotomus, Sergentomvia. Brumptomyia e Lutzomyia. este último com nove subgèneros: Lutzomyia s. str. Dampfomyia, Pintomyia, Pressatia, Evandromyia, liannamvia. Trichophoromvia. Nyssomvia e Psychodopygus

VARGAS (1978) criou outros dois subgèneros para Lutzomyia: Forattiniella e Agualioi

MARTINS e col. (1978) combinando as classificações de BARRETTO (1962), THEODOR (1965) e YOUNG e FAIRCHILD (1974) consideraram um maior número de categorias subgenéricas. Aceitaram quatro gêneros: Brumptomyia, Hertigia, Lutzomvia e Warilevia. Para Luzomyia adotaram dezesseis subgèneros: Lutzomyia s. str. Pintomyia, Evandromyia, Psychodopygus, Viannamyia, Pressatia, Dampfomyia, Micropygomyia, Helcocyrtomyia, Trichophoromyia, Coromyia, Nyssomyia, Psathyromyia, Trichopygomvia, Pifanomyia, Barrettomyia, além de grupos e séries de especies

Em 1984, YOUNG e ARIAS criaram o grupo microps para o gênero Lutzomyia. ARTEMIEV (1991) recorreu à utilização de categorias supra genéricas, dividindo a subfamilia Phlebotominae em duas tribos, sete subtribos, vinte e quatro gèneros, quarenta subgêneros e setenta grupos de espécies. Para a tribo Idiophlebotomini propôs duas subtribos: Hertigiina criada para os gêneros Hertigia e Warileya e Phlebotomini com cinco subtribos, uma destas, Brumptomyiina composta por 10 gèneros: Brumptomyia, Isolutzomyia Artemiev 1991, Psychodopygus, Nyssomyia, 
Irichophoromyia, Psathyromyia, Viannamyia, Pintomyia, Lutzomyia e Micropygomyia. Dividiu Lutzomyia em oito subgêneros: Lutzomvia $s$. str. com três grupos de espécies, Helcocyrtomyia com très grupos de espécies. Barrettomyia com quatro grupos de especies, ('oromyia com seis grupos. Evandromyia com dois grupos. Pressatia, Trichopygomyia e Dampfomyia.

YOUNG e DUNCAN (1994), em trabalho que resultou em guia para identificação das espécies de flebotomineos americanos pertencentes ao gênero Lutzomyia, destacaram a importância médica de Lutzomyia $s$. str. pelo fato de muitas de suas especies apresentarem alto grau de antropofilia e, algumas delas, estarem associadas à transmissão de Leishmaniose. Os autores consideraram três gèneros para os flebotomineos americanos: Brumptomvia, Warileya e Lutzomyia e três para os do Velho Mundo: Phlebotomus, Sergentomyia e Chinius Leng 1987. O gênero Lutzomyia com cerca de 400 espécies foi dividido em quinze subgèneros: ('oromyia, Dampfomyia, Evandromyia, Helcocyrtomyia, Lutzomyia s. str., Micropygomyia, Nyssomyia, Pimtomyia. Pressatia, Psathyromyia. Psychodopygus, Sciopemyia. Trichophoromyia, Trichopygomyia e liannamyia.

GALATI \& CÁCERES (1994) propuseram as séries osornoi, sanguinaria e peruensis para o subgènero Helcocyrtomyia.

GALATI (1995) propòs uma classificação pela metodologia da sistemática filogenética. Apresentou para a subfamilia Phlebotominae, com atenção especial aos táxons da América, uma classificação com 2 tribos, Hertigiini e Phlebotomini. A primeira dividiu em duas subtribos: Hertigiina com dois gèneros, Warileva e Hertigia, e Idiophlebotomina Artemiev, 1991 com cinco gèneros: Spelaeophlebotomus Theodor, 
1948. Idiophlebotomus Quate \& Fairchild, 1961, Phlebotomites Hennig, 1972 e Phlebotomiella Meunier, 1905 e ('hinius. A tribo Phlebotomini foi dividida em seis subtribos: Phlebotomina. Australophlebotomina Artemiev, 1991, Brumptomyiina Artemiev, 1991, Sergentomyiina Artemiev, 1991, Lutzomyiina e Psychodopygina. Para a subtribo Brumptomyiina considerou dois gêneros: Brumptomyia e Oligodontomyia Galati. 1995 Em Sergentomyiina inseriu os gèneros Sergentomyia, Deanemyia Galati, 1995. Micropygomvia (com três novos subgêneros: Sauromyia Artemiev 1991, Silvamyia Galati, 1995 e (oquillettimyia Galati, 1995). Para a subtribo Lutzomyiina Abonnenc \& Leger. 1976 adotou os gèneros Sciopemyia. Lutzomvia, Migonemyia Galati. 1995, Pintomyla. Dampfomyia, Expapillata Galati, 1995, Pressatia, Trychopygomyia, Lvandromvia. A subtribo Psychodopygina Galati. 1995 ficou com sete gêneros: Psatḥ̣romyia (com os subgêneros Psathyromyia s. str., Forattiniella e Xiphomyia), Liamnamyia. Martinsmyia, Bichromomyia Artemiev, 1991, Psychodopygus, Nyssomyia, Trichophoromyia. O gènero Lutzomyia ficou composto por quatro subgèneros: ('astromyia, Helcocyrtomyia, Lutzomyia s. str. e Tricholateralis Galati, 1995

Após esta última proposição, os estudos têm se voltado para a identificação de marcadores moleculares a serem utilizados em abordagens filogenéticas entre alguns grupos de espécies (BOOTH e col. 1996, PEIXOTO e col. 2001, MAZZONI e col 2002, LINS e col 2002, TORGERSON e col 2003, BEATI e col 2004), inclusive as do Velho Mundo (ESSEGHIR e col. 1997, RISPAIL e LEGER 1998a, 1998b, DEPAQUIT e col. 1998, DEPAQUIT e col. 2000, DI MUCCIO 2000)

Em 2003 foi criado um novo gènero monoespecifico pertencente à tribo 
Phlebotomini e não inserido em nenhuma das subtribos: Edentomyia Galati, AndradeFilho, Silva e Falcão, 2003. 
OBJETIVOS 


\section{Objetivos}

\subsection{Objetivo Geral}

Elaborar hipótese de parentesco entre as espécies do gênero Lutzomyia

\subsection{Objetivos específicos}

Verificar se o clado denominado gênero Lutzomyia é, de fato, monofilético.

Investigar se Lutzomyia é constituido por 4 subgrupos monofiléticos que correspondem a subgrupos designados como subgêneros Helcocyrtomyia, Castromyia, Tricholateralis e Lutzomyia s. str.

Investigar se o subgrupo designado como Helcocyrtomyia é constituido por subgrupos monofiléticos designados como séries osornoi, sanguinaria e peruensis. 


\section{JUSTIFICATIVA}




\section{Justificativa}

As fêmeas de diversas espécies de flebotomíneos são reconhecidas como vetores de Leishmaniose e Bartonelose. Alguns grupos de mamíferos selvagens, sinantropicos e domesticos podem ser reservatórios do parasita, assim como o homem em algumas áreas do Velho Mundo. Neste continente, as espécies de flebotomineos associadas à transmissão das Leishmanioses pertencem ao gênero Phlebotomus e na América estão distribuidas em gèneros das subtribos Lutzomyiina e Psychodopygina (GALATI 1995, 2003). No gênero Lutzomyia várias são as espécies implicadas (CIPA Group 1999).

Dentre as especies comprovadamente vetores estão Lutzomyta longipalpis, o principal vetor de Leishmaniose Visceral na América. Além desta, no Brasil, outras como L. forattinii, muito antropofilica e $L$. cruzi já foram apontadas como vetores em Corumbá e Ladário, Mato Grosso do Sul. (GALATI e col 1997, NUNES e col. 1998). Em 1998, SANTOS e col relataram o encontro de Lutzomyia cruzi naturalmente infectada por Leishmamia chagasi Cunha e Chagas, 1937 em Corumbá.

Outras espécies do gènero tèm sido identificadas como possiveis transmissoras de Leishmania $L$ diabolica, $L$. hartmanni. $L$. gomezi, $L$ lichyi, $L$ peruensis, $L$. spathotrichia (CIPA Group 1999)

$\mathrm{Na}$ Colômbia, as espécies L. cirrita, L. erwindonaldoi, L. gomezi, L. hartmanni, L. lichyi, $L$ osornoi, $L$ sanguinaria, $L$. scorzai, $L$. strictivilla, $L$. sp de Pichinde, $L$. araracuarensis, $L$. bifoliata, $L$ marinkellei e $L$. falcata são consideradas de importància medica devido ao alto grau de antropofilia e pela suspeita de transmitirem a forma cutânea de Leishmaniose (MONTOYA-LERNA e FERRO 2003) 
HASHIGUCHI e GOMEZ (1991) identificaram L. ayacuchensis infectada naturalmente com Leishmania mexicana em área de foco endêmico de Leishmaniose no Equador.

No Peru as espécies $L$. peruensis, L. tejadai, $L$. noguchii e também $L$. ayacuchensis tiveram sua presença e abundância associadas à transmissão de Leishmaniose cutânea (CÁCERES e col. 1995, 2000, DAVIES e col. 1997).

Outra importante afeção, que tem a participação dos flebotomineos, é a Bartonelose ou verruga peruana, cujo agente é a bactéria Bartonella bacilliformis Strong, 1913. Esta doença ocorre no Peru, Equador e Colômbia e caracteriza-se por apresentar duas fases: bacteremia aguda acompanhada de febre e anemia profunda e fase crònica com lesões nodulares na pele ou verrugas. Os casos não tratados são fatais e podem representar mais de $40 \%$ dos registros. As espécies $L$. noguchii e $L$. peruensis foram consideradas como potenciais vetores no Peru (BARBARA e col. 1998, VILLASECA e col. 1990).

A correta identificação de vetores e de potenciais vetores de parasitas é fundamental para a compreensão de seu possivel papel na transmissão de agentes (MARCONDES 1998, 2001). Assim sendo, estudos que resgatem a relação de parentesco entre as espécies podem contribuir para a compreensão das relações entre vetores, parasitas e hospedeiros. 


\section{MATERIAL E MÉTODO}




\section{Material}

\subsection{Obtenção e preparo do material utilizado}

Os adultos machos e fêmeas de flebotomíneos foram obtidos mediante empréstimo da Coleção do Departamento de Epidemiologia e do Laboratório de Flebotomíneos da Faculdade de Saúde Pública da Universidade de São Paulo, São Paulo, SP e do Centro de Pesquisa René Rachou da Fundação Oswaldo Cruz, Belo Horizonte, MG. A relação do material examinado e respectiva procedência, bem como o elenco de espécies que fizeram parte desse estudo (grupo interno e externo) podem ser verificadas em anexo (Anexos A-1 a A-11 e A-12 a A-14).

$\mathrm{O}$ número de exemplares analisado para cada espécie variou. $\mathrm{Na}$ falta de representante da espécie utilizou-se dado da literatura.

Todas as medidas de caracteres morfológicos foram submetidas a análise de variância e os intervalos de confiança calculados. Os valores estão apresentados em tabelas e gráficos em anexo (Tabelas 1 a 18, Anexos A-25 a A-42). Os espécimens oriundos de empréstimos de coleção estavam montados em lâminas, segundo posições convencionais que permitem visualizar as estruturas morfológicas. Os exemplares coletados seguiram o mesmo padrão de montagem. $\mathrm{O}$ preparo das lâminas seguiu o protocolo do laboratório:

- Fenol 24 horas

- Hidróxido de potassa a $10 \%, 12$ horas 
- Ácido acético a $10 \%$, lavagem rápida

- Ácido acético a $10 \%+2$ ou 3 gotas de fuccina ácida- 15 minutos

- Álcool $70^{\circ} \mathrm{GL}-10$ minutos

- Álcool a $80^{\circ} \mathrm{GL}-10$ minutos

- Álcool a $90^{\circ} \mathrm{GL}$ - 10 minutos

- Álcool a $95^{\circ} \mathrm{GL}$ - 10 minutos

- Álcool absoluto - 10 minutos

- Eugenol ou Creosoto de Faya - até diafanizar.

- Montagem em lâmina e laminula em Bálsamo

$\mathrm{O}$ número de exemplares analisado de cada espécie, macho ou fềmea, variou segundo o material disponível. Os dados de algumas espécies foram obtidos das descrições originais, de artigos de revisão ou de outros tipos de artigos que se referiam à espécie ou grupo em questão

\section{2 Métodos}

O estudo baseou-se exclusivamente na observação de caracteres morfológicos de machos e fêmeas de flebotomineos. 


\subsubsection{Morfometria}

As observações foram feitas em Microscópio Zeiss $®$ ICS Standard 25 dotado de ocular micrométrica calibrada segundo escala milimétrica padrão de lâmina aferidora. As medidas foram realizadas no mesmo padrão das descrições das espécies. (Figura Anexo A-).

\subsection{Metodologia de análise dos dados morfométricos}

Os valores da morfometria, bem como das relações entre as medidas dos caracteres, foram submetidos a Análise de Variância. Esta análise foi feita com a utilização de programa desenvolvido para este fim ${ }^{1}$. A comparação entre as médias e obtenção dos valores de intervalo de confiança realizada utilizando-se a seguinte fórmula (SOKAL \& ROHLF, 1981):

$$
\begin{aligned}
& \lim =\overline{y i} \pm \sqrt{1 / 2} m_{\left[k^{*}, v \mid\right.} s \overline{y i} \text { sendo: } \\
& \bar{y}=\text { valor médio da medida obtida para a estrutura (obtido pelo programa de }
\end{aligned}
$$
análise de variância)

m = valor critico obtido da Tabela 21 - Valores críticos "studentized" de ROHLF \& SOKAL, 1981

s $\overline{y i}=$ erro padrão (obtido pelo programa de análise de variância) 
$\mathbf{k}^{\star}=$ número de espécies

$v=$ graus de liberdade dentro do tratamento dado por $n-\mathrm{k}^{*}$

$\mathbf{n}=$ número total de observações

O erro padrão obtido pela análise de variância, multiplicado pelo resultado da fórmula e somado e subtraído da média fornece o intervalo de confiança das médias das medidas dos caracteres morfológicos. Esses dados foram submetidos ao programa "Microsoft@ Excel 97 SR1 Copyright O 1983-1997 Microsoft Corporation" para a obtenção dos gráficos, cujas curvas permitiram a determinação dos intervalos de classe de cada uma das medidas obtidas

\subsubsection{Análise cladística}

Para a elaboração da hipótese de parentesco entre as espécies de Lutzomyia utilizou-se a metodologia cladística (HENNIG 1966, AMORIM 1997).

Os sessenta e três caracteres foram polarizados segundo o método de comparação com o grupo externo (WATROUS e WHEELER 1981, MADDISON e col 1984, NIXON e CARPENTER 1993).

A matriz (Tabela 19, Anexos, A-43 e A-44) foi editada com o programa NEXUS versão 0.5.0. (PAGE 2001). Na matriz, "0" (zero) indica o estado plesiomórfico de um caráter; "1" (um), "2" (dois), "3" (três) e "4" (quatro) indicam estados apomórficos para um caráter; "?" indica caracteres não observados em algum táxon; "-"

'Prof. Dr. Jair Licio Ferreira dos Santos. Departamento de Medicina Social de Faculdade de Medicina de Ribeirào Preto-USP. FONE +55 (16) $6023070 / 6025839$ - FAX +55 (16) 6331386. 
indica caracteres não comparáveis.

A análise cladística foi efetuada com o programa "PAUP*4.0 - Win32 /DOS Beta Version 10" (SWOFFORD 2000). O critério utilizado foi de busca heurística, sequência de adição de táxons randômica. Todos os caracteres com múltiplos estados apomórficos foram tratados como não aditivos e não ordenados. Foi empregado o método de aproximações por pesagens sucessivas (FARRIS 1969, CARPENTER 1988, 1994). A topologia do cladograma resultante foi obtida com o programa TreeView 1.5 (PAGE, 1996). Na análise dos estados dos caracteres e na discussão do cladograma utilizou-se a notação de "grupo+" proposta por AMORIM (1982).

\subsection{Ilustração}

As figuras que representam as modificações nos estados dos caracteres analisados foram obtidas a partir de desenho em Microscópio Óptico Olympus $₫$ dotado de câmara clara e por fotomicrografia, diretamente das lâminas estudadas, em Microscópio Zeiss $®$, dotado de câmera fotográfica digital Nikon®. 


\section{RESULTADOS E DISCUSSÃO}




\section{Resultados e Discussão}

\subsection{Análise dos caracteres}

O gênero Lutzomyia, segundo a classificação adotada (GALATI 1995) compreende 69 espécies. Destas foram analisadas sessenta e duas, pois $L$. manciola Ibáñez-Bernal, 2001, L. tanyopis Young \& Perkins, 1984, L. vargasi (Fairchild \& Hertig, 1961), L. botella (Fairchild \& Hertig, 1961), L. erwindonaldoi (Ortiz, 1978) e L. imperatrix (Alexander, 1944) não foram incluidas no estudo, não só por falta de material disponivel, mas também pelo fato de serem insuficientes as informações da literatura. A espécie L. chotensis Galati, Cáceres \& Zorrila, 2003 também não fez parte do conjunto de espécies analisado pelo fato de ter sido descrita somente pelo macho e por faltar no espécime-tipo, único exemplar da espécie, várias estruturas que fazem parte dos caracteres utilizados na análise.

Sessenta e três caracteres foram analisados. Os caracteres l a 18 correspondem a medidas de estruturas da cabeça para machos e fêmeas. Algumas das espécies foram descritas somente pelo macho (L. castroi, L. caligata, L. adamsi, L. wattsi, $L$. araracuarensis e $L$. flabellata) e por isso os caracteres que dizem respeito apenas às fêmeas foram codificados, para essas espécies, como não observados ("?"). Os caracteres 55 a 60 , que se referem à presença ou ausência de papilas tergais nos tergitos abdominais de machos, só puderam ser codificados quando se dispunha do espécime para observação, uma vez que a maioria dos autores não faz alusão a essa estrututa quando descreve ou redescreve espécies. Dessa forma, para L. adamsi, $L$ araracuarensis, $L$. ceferinoi, $L$. cirrita, L. evangelistai, L. flabellata, L. larensis, $L$. 
legerae, L. osornoi, L. stricitivilla, L. tortura, L. marinkellei e L. legerae esses caracteres foram também codificados como não observados ("?").

O resultado da análise de variância e os intervalos de confiança das medidas dos caracteres morfológicos (caracteres 1 a 18) estão apresentados nas tabelas e gráficos em anexo (Tabelas 1 a 18, Figura 1 a18; Anexos A-25 a A-42 e A-45 a A-62).

O cladograma de consenso semi-estrito (Figura19A, Anexo A-63) revelou a formação de dois grupos monofiléticos em Lutzomyia (Figura 19B, Anexo A-64) que foram designados, já na análise dos caracteres, como subgêneros: Lutzomyia s. str. (Figura 19C, Anexo A-65) e Helcocyrtomyia (Figura 19C, Anexo A-66). Ambos contêm grupos monofiléticos tratados como séries de espécies. As de Lutzomyia s. str. foram denominadas como: série longipalpis para L. souzalopesi-, série castroi para $L$. castroi+, série cruciata para L. falcata+ e série cavernicola para L. bicormuta+; as de Helcocyrtomyia foram denominadas como série noguchii, série nova para L. blancasi-; série caballeroi, série nova para L. caballeroit, e série sanguinaria para L. strictivilla+.

\section{Caracteres}

$(0$ = Plesiomórfico; 1, 2, 3, 4 = Apomórfico $)$

Caráter 1. Proporção entre os comprimentos do clipeo e cabeça - macho (Tabela 1. Anexo A-25; Figura 1, Anexo A-45; Figura 20, Anexo A-67; Figura 21 A, B e C, Anexo A68):

$$
\begin{array}{ll}
0 & 0,157-0,177 \\
1 & 0,22-0,305
\end{array}
$$




$$
\begin{array}{ll}
2 & 0,312-0,359 \\
3 & 0,363-0,396
\end{array}
$$

A cabeça dos flebotomineos é grande e subesférica em seu estado plesiomórfico, estado presente nos demais táxons de Psychodidae (Figura 21A, Anexo A-68). O clipeo está entre a face, região delimitada dorsalmente pela área de inserção das antenas e olhos, e o labrum. No estado plesiomórfico o clipeo é curto (Figura 21A, Anexo A-68). A relação entre os comprimentos da cabeça e clipeo apresentou-se como multi-estado em machos e fêmeas. Nos machos pode se considerar que a modificação do estado plesiomórfico para o estado 3 (clipeo longo) (Figura 21C, Anexo A-68, ) está presente no ancestral de Lutzomyia. Assim sendo, em Luzomyia s. str houve modificação para o estado 2 em L. bifoliata+ com modificação para o estado 1 em L. amaralit da série castroi, e em L. evangelistai e L. flabellata+ da série cruciata; na série cavernicola a modificação para o estado 2 está presente em L. bicormuta. No subgênero Helcocyrtomyia o clipeo longo (estado 3) modificou-se para o estado 2 no ancestral de L. osornoi- com reversão para o estado $3 \mathrm{em}$ l. munaypata+ da série caballeroi. O estado 1 foi observado em $L$. osornoi e, na série sanguinaria em $L$. gonzaloi+ com reversão para o estado 2 em L. sanguinaria e $L$. guderiani; nesta mesma série o estado 3 (clipeo longo) está presente em L. adamsi.

Carater 2. Propcrção entre os comprimentos do clipeo e cabeça - fêmea (Tabela 2. Anexo A-26; Figura 2, Anexo A-46; Figura 20, Anexo A67; Figura 21A, B e C. Anexo A68):

$$
0 \quad 0,168-0,275
$$


$1 \quad 0,297-0,349$

$2 \quad 0,35-0,4$

Nas fêmeas de Lutzomyia, clipeo longo pode ter sido o estado presente no ancestral (estado 2). Em Lutzomyia s. str. observou-se o encurtamento representado pelo estado 1 em L. gaminarai da série longipalpis; em L. lichyi+ com reversão para o estado 2 em L. marinkellei e L. legerae da série cruciata e em $L$. renei da série cavernicola. No subgênero Helcocyrtomyia a modificação para o estado l ocorreu em $L$. castanea e $L$. ceferinoi da série caballeroi; em L. hartmanii + da série sanguinaria com reversão para o estado 2 em $L$. cirrita e $L$. adamsi.

Caráter 3. Razão entre os comprimentos dos olhos e cabeça - macho (Tabela 3, Anexo A-27; Figura 3, Anexo A-47; Figura 21 A, Be C, Anexo A68):

$$
\begin{array}{ll}
0 & 0,694-0,713 \\
1 & 0,46-0,624 \\
2 & 0,413-0,45 \\
3 & 0,31-0,395
\end{array}
$$

Em flebotomineos, olhos grandes ocupando mais do que $60 \%$ da cabeça representam o estado plesiomórfico. A redução dos olhos corresponde ao estado derivado. Nos machos, possivelmente o estado 2 estivesse presente no ancestral de Lutzomyia e as modificaçõespara o estado 1 foram verificadas em $L$. forattinii, $L$. almerioi e L. renei da série cavernicola e em L. ischnacantha+. Em Helcocyrromyia. olhos pequenos (estado 3) possivelmente tenha sido o estado presente no ancestral do subgènero e, assim sendo, verificou-se que 2 derivações aconteceram: para o estado 2 
em $L$. pescei+, e para o estado 1 em $L$. galatiae+ com reversão para o estado $2 \mathrm{em} L$. caballeroi da série caballeroi.

Caráter 4. Razão entre os comprimentos dos olhos e cabeça - fêmea (Tabela 4, Anexo A-28; Figura 4, Anexo A-48; Figura 21 A, Be C, Anexo A68):

$$
\begin{array}{ll}
0 & 0,623-0,643 \\
1 & 0,547-0,591 \\
2 & 0,46-0,54 \\
3 & 0,299-0,45
\end{array}
$$

As modificações no comprimento dos olhos das fềmeas podem ser consideradas da seguinte forma: no ancestral de Lutzomyia o estado 2 estava presente. Em Lutzomyia s. str. houve modificação para o estado 1 na série longipalpis, com reversão para o estado 2 em L. alencari+; na série castroi houve derivação para o estado 3 na única fêmea descrita para o grupo, L.amarali; a modificação para o estado 1 é uma apomorfia da série cruciata, na série cavernicola está presente o estado 3 com reversão para o estado 2 em L. forattinii e L. almerioi. No subgênero Helcocyrtomyia manifestou-se o estado 3 no ancestral do subgênero e as alterações que ocorreram foram as seguintes: estado 2 em L. tejadai e L. quillabamba; em L. larensis, L. herreri e L. rispaili da série caballeroi e ainda em L. gonzaloi. Na série sanguinaria as derivações observadas foram: estado 1 em $L$. hartmanni+ com modificação para o estado 2 em $L$. sp de Pichinde e para o estado 3 em $L$. cirrita; no ancestral de $L$. scorzai+ observou-se o estado 2, porém com o estado 1 presente em $L$. guderiani. Alteração para o estado 1 ocorreu também em $L$. munaypata da série caballeroi. 
Caráter 5. Razão entre os comprimentos do labroepifaringe e cabeça- macho: (Tabela 5, Anexo A-29; Figura 5, Anexo A-49; Figura 20, Anexo A-67):

$$
\begin{array}{ll}
0 & 0,142-0,248 \\
1 & 0,415-0,609 \\
2 & 0,623-0,711 \\
3 & 0,723-0,776 \\
4 & 0,819-0,845
\end{array}
$$

No estado plesiomórfico o labroepifaringe é curto. A relação entre os comprimentos desta estrutura e da cabeça revelou quatro classes para as espécies de Lutzomyia. Nos machos, a modifcação para o estado 2 pode ter ocorrido no ancestral de Lutzomyia. Assim sendo, em Lutzomyia s. str. houve modificação para o estado $1 \mathrm{em} L$. ischnacantha; em L. souzalopesi, L. cruzi e L. longipalpis+ da série longiplapis; em L. dispar; em $L$. falcata e $L$. marinkellei+ com reversão para o estado 2 em $L$. legerae e $L$. sherlocki da série cruciata. Em Helcocyrtomyia o estado 3 está presente no ancestral de pallidithorax + com reversão para o estado 2 em L. pallidithorax; em L. caballeroi+ (série caballeroi ) e $L$. hartmanni+ da série sanguinaria; o estado 4 está presente em $L$. wattsi, L. scorzai e $L$. adamsi da série sanguinaria e o estado 1 foi observado em $L$. cirrita e L. tortura desta mesma série.

Caráter 6. Razão entre os comprimentos do labroepifaringe e cabeça - fềmea (Tabela 6, Anexo A-30; Figura 6, Anexo A-50; Figura 20, Anexo A-67):

$$
\begin{array}{ll}
0 & 0,156-0,262 \\
1 & 0,452-0,534
\end{array}
$$




$$
\begin{array}{ll}
2 & 0,589-0,815 \\
3 & 0,825-0,929 \\
4 & 0,959-0,997
\end{array}
$$

Nas fêmeas de Lutzomyia pode se considerar que a modificação para o estado 2 esteve presente no ancestral do gênero. Em Lutzomyia s. str. o estado 3 está presente em L. bifoliata, L. dispar e L. almerioi+ e o estado 1 em L. lichyi e L. carvalhoi. No subgênero Helcocyrtomyia a modificação para o estado 3 ocorreu em L. pallidithorax+, tendo havido, neste mesmo grupo, derivação para o estado 4 em $L$. ceferinoi da série caballeroi; reversão para o estado 2 em L. pallidithorax e L. strictivilla, modificação para o estado 4 em $L$. sp de Pichinde+ com reversão para o estado 2 em L. tortura.

Caráter 7. Proporção entre os comprimentos do segundo palpômero e palpo macho (Tabela 7, Anexo A-31; Figura 7, Anexo A-51; Figura 20, Anexo A-67):

$$
\begin{array}{ll}
0 & 0,103-0,144 \\
1 & 0,149-0,186 \\
2 & 0,191-0,239
\end{array}
$$

Os palpos maxilares no plano básico de Diptera são formados por cinco segmentos (Figura x2, Anexo A-x3). O primeiro artículo é bastante reduzido em relação aos demais. De acordo com o comprimento de cada um dos cinco palpômeros designa-se a fórmula palpal segundo a ordem crescente dos comprimentos. No estado plesiomórfico a fórmula palpal é $1,2,3,4,5$. Houve diminuição no comprimento do quarto palpômero e aumento nos do terceiro e segundo e com isso a fórmula passou para $1,4,2,3,5$ ou $1,(4,2), 3,5$ ou $1,4,(2,3) 5$. Considerando-se a presença do estado 1 no ancestral de 
Lutzomyia, verifica-se que nos machos de Lutzomyia $s$. str. houve diminuição do segundo palpômero em relação ao ancestral em L. alencarit da série longipalpis e um aumento no comprimento (estado 2) em L. araracuarensis + da série cruciata. Se o estado 2 estava presente no ancestral de Helcocyrtomyia, observa-se que as modificações para o estado 1 ocorreram em L. blancasi e L. noguchii da série noguchii; em $L$. osornoi; no ancestral da série caballeroi com reversão para o estado 2 em $L$. munaypata-; em L. scorzai da série sanguinaria.

Caráter 8. Proporção entre os comprimentos do segundo palpômero e palpo fêmea (Tabela 8, Anexo A-32; Figura 8, Anexo A-52; Figura 20, Anexo A-67)

$$
\begin{array}{ll}
0 & 0,105-0,130 \\
1 & 0,147-0,244 \\
2 & 0,246-0,252
\end{array}
$$

Nas fêmeas de Lutzomyia provavelmente o estado I estivesse presente no ancestral e, assim sendo, em Lutzomyia s. str. a derivação para o estado apomórfico 2 ocorreu em L. carvalhoi da série cruciata. Em Helcocyrtomyia o estado 2 foi evidenciado em L. quillabamba, L. castanea, L. ceferinoi, L. munaypata e L. guderiani+

Caráter 9. Proporção entre os comprimentos do terceiro palpômero e palpo macho (Tabela 9. Anexo A-33; Figura 9, Anexo A-53; Figura 20, Anexo A-67)

$$
\begin{array}{ll}
0 & 0,148-0,173 \\
1 & 0,175-0,203 \\
2 & 0,205-0,243
\end{array}
$$


O aumento do terceiro palpômero refletido na proporção que equivale ao estado 1 é uma modificação presente, provavelmente, no ancestral de Lutzomyia. Nos machos de Lutzomyia s. str. ocorreram as seguintes alterações: estado 2 em L. bifoliata; em $L$. souzalopesi da série longiplapis; na série castroi; em L. evangelistai, L. carvalhoi, L. legerae e $L$. sherlocki da série cruciata; em L. battistinii da série cavernicola. Na série cruciata houve modificação para o estado plesiomórfico em $L$. araracuarensis. No subgênero Helcocyrtomyia foi evidenciado o estado 2 nas espécies da série noguchii; em L. osornoi e em L. galatiae--; o estado $3 \mathrm{em}$ L. kirigetiensis com reversão para o estado 2 em $L$. sp de Pichinde e L. adamsi- da série sanguinaria.

Caráter 10. Proporção entre os comprimentos do terceiro palpômero e palpo fêmea (Tabela 10, Anexo A-34; Figura 10, Anexo A-54; Figura 20, Anexo A-67):

$$
\begin{array}{ll}
0 & 0,15-0,162 \\
1 & 0,176-0,243 \\
2 & 0,25-0,305
\end{array}
$$

Nas fêmeas de Lutzomyia s. str. a modificação para o estado 2 ocorreu em $L$. amarali da série castroi. No subgênero Helcocyrtomyia são as espécies da série sanguinaria que apresentam a modifcação.

Caráter 11. Proporção entre os comprimentos do quarto palpômero e palpo macho (Tabela 11, Anexo A-35; Figura 11, Anexo A-55; Figura 20, Anexo A-67):

$0 \quad 0,155-0,203$ 
$1 \quad 0,103-0,153$

A derivação do estado plesiomórfico em machos de Lutzomyia pode ter ocorrido da seguinte forma: no subgènero Lutzomyia s. str. o estado 1 foi observado em $L$. souzalopesi da série longipalpis, em $L$. lichyi, na série cruciata e em $L$. almerioit da série cavernicola; no subgênero Helcocyrtomyia a modificação para o estado 1 ocorreu no ancestral de L. avacuchensis+ e de $L$. pescei+ com reversão em L. galatiae

Caráter 12. Proporção entre os comprimentos do quarto palpômero e palpo fêmea (Tabela 12, Anexo A-36; Figura 12, Anexo A-56; Figura 20, Anexo A-67)

$$
\begin{array}{ll}
0 & 0,171-0,207 \\
1 & 0,134-0,165 \\
2 & 0,103-0,129
\end{array}
$$

Nas fèmeas de Lutzomyia pode se considerar que o estado 1 estivesse presente no ancestral do gènero e dessa forma, a modificação observada foi aumento do quarto palpômero em L. gaminarai da série longipalpis. Em Helcocyrtomyia houve uma derivação para o estado 2 no ancestral das séries caballeroi e sanguinaria.

Caráter 13. Proporção entre os comprimentos do quinto palpômero e palpo macho (Tabela 13, Anexo A-37; Figura 13, Anexo A-57; Figura 20, Anexo A-67):

$$
\begin{array}{ll}
0 & 0,448-0,504 \\
1 & 0,356-0,444 \\
2 & 0,199-0,346
\end{array}
$$

Nos machos de Lutzomyia observou-se modificação para o estado 1 em $L$. 
bifoliata+ com reversão em $L$. alencari+ da série longiplapis e em e L. lichyi; na série castroi houve modificação para o estado 2 em L. caligata; na série cruciata o estado 2 está presente em $L$. falcata, $L$. diabolica e $L$. legerae com reversões para o estado plesiomórfico em L. gomezi, L. carvalhoi, L. spathotrichia e L. flabellata+; na série cavernicola o estado 1 ocorreu em L. battistiniit com reversão para o estado plesiomórfico em L. cavernicola + . Em Helcocyrtomyia, com provável ocorrência do estado 1 no ancestral do subgènero, verificou-se reversão em L pallidithorax e modificações para o estado 2 em L. quillabamba, em L. ceferinoi+ da série caballeroi e nas seguintes especies da série sanguinaria: L. hartmanni. L. kirigetiensis, $L$. sanguinaria e L. tortura.

Caráter 14. Proporção entre os comprimentos do quinto palpômero e palpo fêmea (Tabela 14. Anexo A-38; Figura 14, Anexo A-58: Figura 20. Anexo A-67)

$$
\begin{array}{ll}
0 & 0,451-0,501 \\
1 & 0.347-0,446 \\
2 & 0.251-0.338
\end{array}
$$

Considerando-se o estado 1 presente no ancestral de Lutzomyia, observou-se que em Lutzomyia s. str: o estado 2 está presente em L. ischnacanta. em L. gaminarai da série longipalpis e L. amarali da série castroi. Neste mesmo subgênero houve modificação para o estado plesiomórfico (estado zero) em L. alencari, L. cruzi e $L$. longipalpis. No subgènero Helcocyrtomyia houve modificação para o estado plesiomórfico (estado zero) em L. chavinensis da série noguchii; o estado 2 foi observado em $L$. pescei - com reversões para o estado I em L. tejadai, em L. caballeroi. 
L. larensis, L. ceferinoi+ e L. rispaili da série caballeroi; na série sanguinaria a reversão para o estado 1 foi verificada em $L$. stricitivilla.

Caráter 15. Razão entre os comprimentos do segundo e quarto palpômeros macho (Tabela 15, Anexo A-39; Figura 15, Anexo A-59; Figura 20, Anexo A-67):

$$
\begin{array}{ll}
0 & 0,51-0,922 \\
1 & 0,962-1,237 \\
2 & 1,258-1,691 \\
3 & 1,847-2,093
\end{array}
$$

Considerando-se o estado 1 presente no ancestral de Lutzomyia verificou-se que houve derivação para o estado plesiomórfico nos machos de $L$. alencarit da série longipalpis; para o estado $2 \mathrm{em}$ L. amarali da série castroi e L. spathotrichia da série cruciata. No subgênero Helcocyrtomyia o estado 2 foi observado em L. chavinensis da série noguchii; em L. ayacuchensis e em L. tejadai+ com reversão para o estado 1 em $L$. ceferinoi da série caballeroi e derivação para o estado $3 \mathrm{em}$ L. sanguinaria e $L$. tortura+ da série sanguinaria

Caráter 16. Razão entre os comprimentos do segundo e quarto palpômeros fêmea (Tabela 16, Anexo A-40; Figura 16, Anexo A-60; Figura 20, Anexo A-67):

$$
\begin{array}{ll}
0 & 0,607-0,695 \\
1 & 0,842-1,337 \\
2 & 1,355-1,791 \\
3 & 1,918-2,435
\end{array}
$$


Considerando-se o estao 1 presente no ancestral de Lutzomyia verificam-se as seguintes modificações: em Lutzomyia s. str. derivação para o estado 2 em L. bifoliata; em L. bicormuta e L. almerioi da série cavernicola. No subgênero Helcocyrtomyia ocorreu modificação para o estado 2 em L. noguchii da série noguchii; em $L$. ayacuchensis+; em L. pescei e em L. quillabamba +. Em L. castanea+ (série caballeroi) está presente o estado 3 com reversão para o estado 2 em $L$. ceferinoi e modificação para o estado plesiomórfico em L. rispaiii. Na série sanguinaria houve reversão para o estado 2 em L. tortura.

Caráter 17. Razão entre os comprimentos do quinto e terceiro palpòmeros macho (Tabela 17, Anexo A-41; Figura 17, Anexo A-61; Figura 20, Anexo A-67):

$$
\begin{array}{ll}
0 & 2,853-3,288 \\
1 & 2,226-2,584 \\
2 & 1,361-2,198 \\
3 & 0,702-1,25
\end{array}
$$

Em ambos os sexos, o estado 1 está presente no ancestral do gênero Lutzomyia. Nos machos de Lutzomyia s. str. verificou-se o estado 2 em L. bifoliata; L. souzalopesi e L. longipalpis da série longipalpis; ainda nesta série observou-se modifcação para o estado 3 em L. gaminarai; o estado 2 está presente também em $L$. dispar+ com possivel reversão para o estado 1 em $L$. lichyi e nas seguintes espécies da série cruciata: $L$. gomezi. L. spathotrichia, L. araracuarensis e L. flabellata. Na série cavernicola houve modificação para o estado 2 em L. battistinii e L. forattinii. No subgênero Helcocyrtomyia considerou-se o estado 2 presente no ancestral do subgênero com 
reversão para o estado 1 em L. pallidithorax; modificação para o estado 3 em $L$. ceferinoi da série caballeroi; e em L. kirigetienis e L. tortura da série sanguinaria.

Caráter 18. Razão entre os comprimentos do quinto e terceiro palpômeros fềmea (Tabela 18, Anexo A-42; Figura 18, Anexo A-62; Figura 20, Anexo A-67):

$$
\begin{array}{ll}
0 & 3,051-3,098 \\
1 & 2,0-2.634 \\
2 & 1,142-1,974 \\
3 & 0.875-1.09
\end{array}
$$

Considerando-se o estado 1 no ancestral de Lutzomyia, verificou-se que a modificação para o estado 2, em Lutzomyia s. str. ocorreu em L. bifoliata; em $L$. souzalopesi e L. longipalpis da série longipalpis; em L. amarali da série castroi; em L. diabolica. L. evangelistai, L. carvalhoi, L. legerae e L. sherlocki da série cruciata, na série cavernicola o estado 2 foi observado em $L$. bicormuta, L. forattinii e $L$. renei. No subgênero Helcocyrtomyia o estado 2 está presente em L. chavinensis + da série noguchii e em $L$. pesceit, na série sanguinaria o estado 3 foi evidenciado em $L$. gonzaloi e em $L$. cirrita+ com reversão para o estado 2 em L. adamsi.

Caráter 19. Espinhos de Newstead no segundo palpômero (PII): macho e fêmea (Figura 22A. Anexo A-69)

$0 \quad$ ausentes

$$
\text { l presentes }
$$

No palpo podem estar presentes os espinhos de Newstead A plesiomorfia 
equivale à presença destes espinhos apenas no terceiro palpômero. Estas estruturas estào presentes no segundo palpômero das espécies da série longipalpis (L. souzalopesi`) e em L. dispar

Caráter 20. Posição dos espinhos de Newstead no terceiro palpômero (PIII) macho e fêmea (Figura 22 B, C e D, Anexos A-69):

0 concentrados no terço basal

1 concentrados na região mediana

2 dispersos pelo segmento

A plesiomorfia equivale à presença dos espinhos de Newstead concentrados no terço basal do terceiro palpômero. Em Lutzomyia a modificação para o estado l está presente no ancestral do gênero. O estado 2, que corresponde à presença dos espinhos de Newstead dispersos pelo terceiro segmento palpal, está presente em $L$. dispar- e em $L$. forattinit de Lutzomyia s. str. e tambèm no ancestral do subgènero Helcocyrtomyia

Caráter 21. Ascóides no primeiro flagelômero (AIII) - macho e fêmea (Figura 20, Anexo A-67)

$0 \quad$ ascóide externo implantado em posição mais basal que o interno 1 ascoide externo implantado em posição mais apical que o interno O par de artenas de flebotomineos, presente na cabeça, é constituido pelo escapo, segmento basal; pedicelo, segundo segmento e catorze flagelòmeros. plesiomorfia encontrada nos demais Nematoceras. No plano básico os segmentos basais são maiores que os distais e o primeiro flagelòmero, denominado AIlI, é bastante longo 
Nos segmentos da antena também podem estar presentes um ou dois espinhos sensoriais denominados ascóides ou espinhos geniculados (Figura 20, Anexos A-67). Estes são semelhantes a cerdas hialinas laminares cujo comprimento é variável, inclusive no nível intraespecífico e podem apresentar ou não prolongamento posterior. Quando presente o par, o nivel de inserção no flagelômero é variável. $\mathrm{O}$ ascóide externo situado em posição mais apical que o interno é sinapomorfia do gènero Lutzomyia.

Caráter 22. Papila peri-ascoidal em AIII - macho e fềmea

$0 \quad$ presente

$1 \quad$ ausente

Em AIII, o estado plesiomórfico corresponde à presença de duas papilas ou sensilas, estruturas sensoriais em forma de roseta no primeiro flgelômero (AIII): uma peri-ascoidal e outra pré-apical (Figura 22 A e B, Anexo A-70). Ausència da papila periascoidal é sinapomorfia do gênero Lutzomyia

Caráter 23. Papila pré-apical em AIII - macho e fêmea

0 presente

1 ausente

As espécies do gênero Lutzomyia apresentam a papila pré-apical Figura $22 \mathrm{~B}$. Anexo A-70)

Carater 24. Papila em AV - macho e fềmea:

$0 \quad$ presente 
1 ausente

No terceiro flagelômero (AV) a condição plesiomórfica corresponde à presença de papila pré-apical. A ausência desta papila foi observada em $L$ falcata , autamoporfia para a série cruciata

Caráter 25. Papila em AXII macho e fêmea:

$0 \quad$ presente

1 ausente

Nos antenômeros apicais AXII a AXVI a plesiomorfia é representada pela presença de papilas apicais, medianas e basais. A ausência destas papilas em AXII é a apomorfia observada nas espécies de Lutzomyia, porém nas espécies da série castroi estas papilas estão presentes.

Caráter 26. Papila em AXIII - macho e fềmea:

0 presente

1 ausente

A ausência de papilas em AXIII é apomorfia de Lutzomyia, mas a presença destas papilas é evidente nas espécies da série castroi, em $L$. bịfoliata e em $L$. cruciata.

Caráter 27. Área esclerosada do cibário - fềmea:

0 ausente

1 presente

A cavidade bucal dos flebotomineos, presente internamente na cabeça. é 
constituida pelo cibário que se une com o labro e hipofaringe pela extremidade anterior, e com a faringe pela posterior. Em linhas gerais, o cibário é formado pela união dos escleritos laterais que se prolongam transversalmente e quando se unem formam a ponte posterior. O teto do cibário pode apresentar uma linha curva denominada protuberância posterior e uma área com pigmentação mais intensa, a área esclerosada do cibário. A presença da área esclerosada do cibário é uma sinapomorfia de Lutzomyia.

Caráter 28. Armadura cibarial: dentes anteriores e posteriores - fềmea (Figura 24, Anexos A-71):

0 ausentes

1 presentes os anteriores e posteriores, horizontais em relação ao lúmen da cavidade bucal

2 presentes os anteriores verticais e posteriores horizontais em relação ao lúmen da cavidade bucal

Na margem posterior do cibário está presente a armadura bucal constituída por dentes que podem formar vários conjuntos: quando implantados junto aos escleritos laterais e orientados em direção ao centro são denominados dentes laterais; quando implantados em posição anterior aos laterais e e em posição horizontal em relação ao lúmen da cavidade bucal são denominados dentes anteriores horizontais; quando os dentes anteriores estão dispostos perpendicularmente em relação ao lúmen da cavidade bucal são denominados dentes anteriores verticais. Além dos dentes anteriores existem os posteriores, sempre em posição horizontal ao lúmen. $O$ estado apomórfico 2 que corresponde à presença dos dentes anteriores verticais e posteriores horizontais relação 
ao lúmen do tubo ocorre no ancestral de Lutzomyia e houve uma modificação para o estado 1 em $L$. amarali, ou seja, na fêmea desta espécie os dentes anteriores estão em posição horizontal em relação ao lúmen

Caráter 29. Número de dentes posteriores (horizontais) no cibário - fèmea

$$
\begin{aligned}
& 0 \text { quatro } \\
& 1 \text { mais do que quatro }
\end{aligned}
$$

A presença de mais do que quatro dentes posteriores (horizontais) no cibário foi evidenciada em L. souzalopesi+, sinapomorfia da série longipalpis (Figura 24E, Anexos A-71)

Caráter 30. Dentes anteriores próximos e laterais à área esclerosada do cibário, mais evidentes que os demais - fêmea (Figura 24F, Anexo A-71)

$0 \quad$ ausentes

1 presentes

Os dentes anteriores proximos e laterais a area esclerosada do cibario, mais evidentes que os demais é uma apomorfia de $L$ forattimii

\section{Caráter 31. Sensila ventro-cervical (Figura 25, Anexo A-72)}

0 presente

1 ausente

Todos os taxons de Lutzomyia apresentam a sensila ventro-cervical no torax 
Caráter 32. Cerdas proepimerais - macho e fêmea (Figura 25, Anexo A-72)

$0 \quad$ ausentes

1 presentes

A presença das cerdas proepimerais no tórax de flebotomíneos é sinapomorfia do gênero Lutzomyia.

Caráter 33. Razão entre o comprimento do primeiro protarsômero e a soma dos comprimentos do segundo ao quinto - macho:

0 menor que 1,0

l maior que 1

As pernas são longas nos Psychodidae seguindo o plano básico de Diptera Compõem-se de cinco segmentos: coxa, trocânter, fềmur, tíbia e tarso. O tarso apresenta cinco tarsômeros e o acrópodo que contém o arólio, estrutura membranosa saculiforme, e as garras tarsais. A apomorfia, que corresponde à relação entre o comprimento do primeiro protarsômero e a soma do segundo ao quinto maior que 1 , foi verificada no ancestral do grupo composto pelas espécies que pertencem ao subgênero Helcocyriomyia.

Càráter 34. Cerdas na região anterior do catepisterno - macho e fềmea (Figura 25, Anexo A-72)

$$
\begin{array}{ll}
0 & \text { presentes } \\
1 & \text { ausentes }
\end{array}
$$

A ausência das cerdas na região anterior do catepisterno é apomorfia presente 
nas espécies de Lutzomyia s. str. e da série noguchii do subgênero Helcocyrtomyia.

Caráter 35. Cerda(s) pós-alar(es) - macho e fêmea (Figura 25, Anexo A-72):

0 presente

1 ausente

A ausência da(s) cerda(s) pós-alar(es) é sinapomorfia observada no ancestral de Lutzomyia.

Caráter 36. Extremidade inicial da nervura $R_{1}$ em relação à da $M_{4}$ - macho e fêmea (Figura 26, Anexos A-73):

$0 \quad$ ultrapassa a de $\mathrm{M}_{4}$

1 coincide com a de $\mathrm{M}_{4}$

2 não atinge a de $\mathrm{M}_{4}$

Inseridas no mesotórax, as asas de flebotomíneos são alongadas, com extremidade apical afilada ou arredondada e com revestimento de cerdas bastante numeroso. Apresentam dez veias paralelas que se ramificam a partir da região basal. A veia transversa $r$-m está entre o terço basal e a região mediana da asa. A asa é composta por seis veias primárias: costa $(C)$, subcosta $(\mathrm{Sc})$, radial $(\mathrm{R})$, média $(\mathrm{M})$, cubital $(\mathrm{Cu})$ e anal (A). C abrange toda a margem alar e assim como Sc não apresenta ramificações. $\mathrm{R}$ apresenta um ramo anterior simples, $\mathrm{R}_{1}$. e um ramo posterior, o setor radial ( $\mathrm{SR}$ ) de onde se originam $R_{5}$ e $R_{2+3+4}$; de $R_{2+3+4}$ origina-se $R_{4}$ e $R_{2+3}$; de $R_{2-3}$ originam-se $R_{2}$ e $R_{3}$. $O$ ramo anterior de $M$ desaparece e posterior origina $M_{1}, M_{2}, M_{3}$ e $M_{4}$. Quanto a $C u$ e $A$, a bifurcação anterior origina $\mathrm{CuA}_{1}$ e $\mathrm{A}_{1}$, e a posterior $\mathrm{CuA}_{2}$ e $\mathrm{A}_{2}$ respectivamente 
Quando se compara a extremidade incial da nervura $R_{1}$ em relação à da nervura $M_{4}$, traçando-se uma perpendicular a $\mathbf{R}_{5}$, observa-se que o estado apomórfico corresponde a um recuo de $R_{1}$ que pode coincidir com a extremidade de $M_{4}$ ou recuar ainda mais, em posição mais proximal que a de $\mathrm{M}_{4}$. Em Lutzomyia s. str. o estado l é uma apomorfia de L. gaminarai+ com reversão para o estado plesiomórfico em $L$. cruzi e derivação para o estado 2 em: L. ischyracantha e L. pseudolongipalpis (série longipalpis), em L. amarali da série castroi e em $L$. cruciatat (série cruciata) com reversão para o estado 1 observada em L. diabolica, L. evangelistai e L. araracuarensis ${ }^{+}$. Em Helcocyrtomyia ocorreram as seguintes modificações em relação ao estado 1 presente possivelmente no ancestral: reversào para o estado plesiomórfico em $L$. pallidithorax, em L. larensis (série caballeroi) e em L. adamsit (série sanguinaria); estado 2 em $L$. ceferinoi e $L$ munaypata+ da série caballeroi e, na série sanguinaria:

L. kirigetiensis e L. scorzai com reversão para o estado 1 em $L$. guderiani e transformação para o estado plesiomórfico em L. adamsit e L. tortura

Carater 37. Nervura $R_{2}$ (alfa) - machos e fèmeas

0 longa, a junção das nervuras $\mathbf{R}_{2} \mathbf{R}_{3}$ localiza-se aquém da extremidade apical de $\mathrm{M}_{4}$

1 mediana, a junção de $R_{2} R_{3}$ coincide com a extremidade apical de $\mathbf{M}_{4}$

2 reduzida, a junção $R_{2} R_{3}$ ultrapassa a extremidade apical de $M_{4}$

A nervura $R_{2}$ em estado plesiomórfico é longa e a derivação corresponde a um encurtamento no comprimento da mesma. A avaliação deste caráter se deu a partir de 
uma perpendicular a $R_{5}$ como para o caráter 36 . O estado apomórfico 1 . nervura $R_{2}$ mediana com a junção de $R_{2} R_{3}$ coincidindo com a extremidade apical de $M_{4}$, foi verificado em Lutzomyia s. str. nas especies $L$. alencari, $L$. ischyracantha e $L$. longipalpis da série longipalpis; em L. dispar; em L. bicormuta da série cavernicola e em L. araracuarensis da série cruciata. $\mathrm{O}$ estado apomórfico 2 , nervura $\mathrm{R}_{2}$ reduzida com a junçào $R_{2} R_{3}$ ultrapassando a extremidade apical de $M_{4}$. foi observado apenas em $L$ battistinii da série cavermicola. No subgênero Helcocyrtomyia o estado 1 está presente somente em L. blancasi da série noguchii.

Caráter 38. Cerdas nas pleuras abdominais (Figura 27, Anexos A-74)

$0 \quad$ ausentes

1 presentes

As pleuras dos Phlebotominae são cobertas por microtriquias e geralmente não apresentam cerdas, porem estas podem ser evidenciadas em $L$. falcata , autapomorfia da série cruciata

Caráter 39. Cerda(s) na região basal da face ventral do gonocoxito (Figura 28. Anexos A-75):

$0 \quad$ presente(s)

1 ausente

Integra a formação da genitália masculina um par de gonocoxitos (Figura 28. Anexos A-75) posicionados lateralmente ao nono tergito. Os gonocoxitos são revestidos por cerdas ou conjunto de cerdas. A apomorfia representada pela ausência de cerda(s) na 
região basal da face ventral do gonocoxito ocorre em $L$. tortura e $L$. adamsi

Caráter 40. Cerda(s) na região basal da face ventral do gonocoxito (Figura $29 \mathrm{~A}$,

B, C e D, Anexos A-76):

0 dispersas

1 compactadas

As cerdas na região basal da face ventral, dispersas pelo gonocoxito, estado plesiomórfico, podem apresentar-se em conjunto compactado. Esta apomorfia está presente no ancestral de Lutzomyia, porém em L. cirrita da série sanguinaria (subgênero Helcoç̣rtomyia) as cerdas não se apresentam compactadas

Caráter 41. Número de cerdas na região basal da face ventral do gonocoxito:

$\begin{array}{ll}0 & 1-5 \\ 1 & 6 \\ 2 & 8 \\ 3 & 9-14 \\ 4 & \text { mais que 14 }\end{array}$

O conjunto de cerdas na região basal da face ventral do gonocoxito derivou para um aumento no número das mesmas. Em Lutzomyia s. str., observou-se o estado $1 \mathrm{em} L$ amaralit; na série cruciata, está presente o estado 3 (9-14 cerdas) com modificação para o estado 1 (6 cerdas) em L. diabolica, L. evangelistai e $L$. carvalhoi e para o estado 2 (8 cerdas) em L. spathotrichia+; nesta mesma série, L. marinkellei apresenta um numero de cerdas maior que 14 (estado 4). Em Helcocyrtomyia o estado 3 está presente em $L$. 
blancasi da série noguchii; o estado 4 foi observado em chavinensis+ também desta série e em $L$. pallidithoraxt com reversão para o estado $3 \mathrm{em}$ L. ayacuchensis- e em $L$ ceferinoi+ da série caballeroi; verificou-se uma redução para 1-5 cerdas em $L$. gonzaloi+ da série sanguinaria.

Caráter 42. Cerda(s) na região basal da face ventral do gonocoxito (Figura 29 A, B, C, D, Anexos A-76):

$0 \quad$ implantada(s) na superficie

1 implantada(s) em capítulo

2 implantada(s) em tubérculo

Quando estão presentes, as cerdas da região basal da face ventral do gonocoxito podem estar implantadas diretamente na superficie do artículo, em capitulo ou tubérculo. Considerando-se o estado 1 como um caráter presente no ancestral de Lutzomyia, foi observado que o estado 2, cerdas implantadas em tubérculo é uma apomorfia de $L$. bifoliata+ do subgênero Lutzomyia s. str. Houve modifcação para cerdas implantadas na superficie no ancestral de L. gaminarai+ (série longipalpis); na série castroi; em $L$. evangelistai da série cruciata e em $L$. renei da série cavernicola. No subgênero Helcocyrtomyia o estado 2 é apomorfia presente no ancestral com modificação para o estado 1 em $L$. pallidithorax+ e para o estado plesiomórfico em $L$. gonzaloi+ .

Caráter 43. Cerda(s) na região basal da face ventral do gonocoxito (Figura 29 A, B, C, D, Anexos A-76):

0 presença de cerda(s) fina(s) somente 
1 presença de cerda(s) semifoliácea(s)
2 presença de cerda(s) foliácea(s)

Nas cerdas da região basal da face ventral do gonocoxito ocorreram modificações quanto ao aspecto das mesmas. $\mathrm{O}$ conjunto de cerdas pode conter cerdas finas (Figura 29A e C, Anexos A-76), semifoliáceas (Figura 29D, Anexos A76) e foliáceas (Figura 29B, Anexos A-76). Em Lutzomyia s. str. a presença de cerdas semifoliáceas (estado 1) foi evidenciada em $L$. souzalopesi e em $L$. cruzi+ da série longiplapis; em $L$ dispar; e nas seguintes espécies da série cruciata: $L$. cruciata, $L$. carvalhoi, L. spathotrichia, L. araracuarensis e L. flabellata. Na série cavernicola estão presentes as cerdas foliáceas (estado 2) com modificação para o estado 1 no ancestral de L. cavernicola ${ }^{+}$. Em Helcocyrtomyia o estado 1 está presente no ancestral do subgênero porém, houve reversão para o estado plesiomórfico em: L. pescei, em L. caballeroi da série caballeroi, em L. ceferinoi+ e na série sanguinaria.

Caráter 44. Cerda(s) bem desenvolvida (s) no terço mediano da face ventral do gonocoxito (Figura 29B, Anexos A-76):

0 ausente

1 presente

A presença de cerda adicional bem desenvolvida no terço mediano da face ventral do gonocoxito foi evidenciada na série cavernicola com reversão em $L$. cavernicolat

Caráter 45. Espinhos apicais do gonóstilo (Figura 30, Anexos A-77) : 
1 presença de um desenvolvido e um atrofiado

2 presença de um espinho desenvolvido

No ápice dos gonocoxitos insere-se um par de gonóstilos. Estes apresentam a superficie coberta por cerdas e contêm um conjunto de espinhos cujo número e disposição modificou-se nos grupos de Phlebotominae. A presença de cinco espinhos dois apicais, um externo superior, um externo inferior e um interno representa o estado plesiomórfico para a subfamília (Figura 30.1 a 30.11, Anexos A-77). A presença de um espinho desenvolvido e um atrofiado é uma apomorfia de L. bifoliata+ (Figura 30.13 e 30.14, Anexos A-77). Em L. dispar e na série cruciata observou-se presença de um espinho apical (estado 2) (Figura 30.15, Anexos A-77)

Caráter 46. Nivel de implantação do espinho interno do gonóstilo (Figura 30, Anexos A-77):

$0 \quad$ além do meio

1 no meio

$2 \quad$ aquém do meio

O deslocamento dos espinhos em direção à base do gonóstilo corresponde à derivação. Para a determinação dos estados desse caráter analisou-se a posição do espinho em relação à metade do comprimento do gonóstilo. No ancestral de Lutzomyia s. str. está presente o estado 1 e houve modificação para o estado plesiomórfico. deslocamento do espinho interno para uma posição mais distal, em $L$. souzalopesi e $L$. gaminarai da série longipalpis. Nesta mesma série observou-se o estado 2 (espinho em 
posição mais proximal) em $L$. alencari, L. ischyracantha e $L$. longipalpis (figura 30.14, Anexos A-77). $\mathrm{O}$ estado 2 está presente também em $L$. caligata da série castroi; em $L$. falcata, L. spathotrichia e L. legerae da série cruciata, e em L. battistinii- da série cavernicola. No subgênero Helcocyrtomyia o estado 2 é apomorfia da série noguchii com o estado 1 presente em L. noguchii; a modificação para o estado 2 ocorreu também em L. pallidithorax; em L. ayacuchensis e em L. cirrita da série sanguinaria. A modificação para o estado plesiomórfico em Helcocyrtomyia ocorreu em L. peruensis; em L. castanea e L. herreri da série caballeroi e L. kirigetiensis da série sanguinaria.

Caráter 47. Nivel de implantação do espinho externo superior do gonóstilo (Figura 30, Anexos A-77):

$$
\begin{array}{ll}
0 & \text { próximo ao quinto apical } \\
1 & \text { próximo ao quarto apical } \\
2 & \text { próximo ao terço apical } \\
3 & \text { no meio }
\end{array}
$$

Para a determinação deste caráter procedeu-se da mesma forma que para o caráter anterior (46). A avaliação da posição do espinho externo superior foi feita em relação à medida do comprimento do gonóstilo. No ancestral de Lutzomyia o espinho externo superior poderia estar posicionado próximo ao quarto apical (estado 1). A partir desta posição observou -se modificação para o estado 2 (espinho posicionado no terço apical) em L. gaminarai, em L. ischyracantha e em L. pseudolongipalpis da série longipalpis. Nas séries castroi e cruciata o espinho está posicionado no quinto apical, porém nesta última série ocorreram as seguintes modificações: reversão para o estado 1 
em L. marinkellei e derivação para o estado 2 em L. carvalhoi Na série cavernicola o estado 2 foi observado em L. battistiniit. No subgênero Helcocyrtomyia o estado 2 está presente na série noguchii, em $L$. pallidithorax, em $L$. osornoi, em $L$. caballeroi, em $L$. gonzaloi, em L. hartmanni, L. sp de Pichinde e $L$. cirrita. O espinho posicionado próximo ao quinto apical foi observado na série peruensis, em L. pescei (Figura 30-4, Anexos A77), L. tejadai (Figura 30.4, Anexos A-77), L. castanea, L. ceferinoi-, $L$. rispaili, L. scorzai e L. tortura.

Caráter 48. Nivel de implantação do espinho externo inferior do gonóstilo (Figura 30, Anexos A-77):

0 além do meio

1 no meio

$2 \quad$ aquem do meio

Para a determinação deste caráter procedeu-se da mesma forma que para os dois caracteres anteriores. A avaliação da posição do espinho externo inferior foi feita em relaçào à medida do comprimento do gonóstilo. Considerando-se que o espinho externo inferior do gonóstilo estivesse implantado em posição mais proximal, estado 2, no ancestral de Lutzomyia, verifica-se que em Lutzomyia s. str. ocorreu uma modificação para o estado l em L. cruzi da série longipalpis; na série castroi e nas seguintes espécies da série cruciata. L. falcata, L. cruciata, L. gomezi, L. marinkellei e L. evangelistai. No subgênero Helcocyrtomyia observou-se o estado 1 em $L$. larensis, L. ceferinoi e $L$. rispaili da série caballeroi, em L. kirigetiensis e L. monzonensis da série sanguinaria. 
Caráter 49. Margem dorsal do parâmero (Figuras 31, 32, 33,Anexos-A-78, A79, A-80):

0 retilinea

1 inflete-se em direção ao gonocoxito

A margem dorsal do parâmero apresentou modificação no seu aspecto retilíneo (Figura 31A, Anexo A-78). Tal modificação se traduz por inflexão em direção ao gonocoxito (Figura 31B, 31D, Anexo A-78). Em Lutzomyia s. str. o estado 1 ocorreu em L. bifoliata+ com reversão para o estado plesiomórfico em $L$. pseudolongipalpis da série longipalpis, em L. diabolica, L. carvalhoi e L. araracuarensis da série cruciata. O estado 1 na série cavernicola é apomorfia de L. forattinii- com reversão em L. renei. No subgênero Helcocyrtomyia o estado 1 está presente nas espécies da série noguchii, em $L$. ayacuchensis, em L. pescei+, em L. galatiae, em L. quillabamba, em L. castanea, em $L$. ceferinoi, em L. munaypata+; em L. strictivilla+ o estado 1 também está presente, porém -com reversão em L. kirigetiensis, $L$. sanguinaria, $L$. sp. de Pichinde e $L$. cirrita e em $L$. monzonensis.

Caráter 50. Espinho(s) na região basal da margem dorsal do parâmero destacado dos apicais:

$$
\begin{array}{ll}
0 & \text { ausente } \\
1 & \text { presente(s) }
\end{array}
$$

Além da cerdosidade presente na margem dorsal do parâmero podem se destacar nesta, espinhos na região basal. A presença (Figura 32F, 32G, 32H, 33I, 33J, 33L, Anexos A-79, A-80) destes espinhos foi evidenciada em Lutzomyia s. str. porém em $L$. 
cruciata+ observou-se ausência (Figura 31 A, 31B, 31C, 31D e 32E, Anexos A-78 e A79) destes espinhos.

Caráter 51. Número de espinhos na região basal da margem dorsal do parâmero:

$\begin{array}{ll}0 & \text { um } \\ 1 & \text { dois } \\ 2 & \text { três } \\ 3 & \geq 4\end{array}$

O número de espinhos na margem dorsal do paràmero é variavel. A presença de dois espinhos (estado 1) (Figura $32 \mathrm{G}$ e $32 \mathrm{H}$, Anexos A-79) provavelmente era o estado presente no ancestral de Lutzomyia, e assim sendo, a redução para 1 espinho ocorreu em L. castroi Figura 33I, Anexos A80) e L. caligata da série castroi (Lutzomyia s. str.) e $L$. falcata (série cruciata). O estado 2 (três espinhos) foi observado em: L. dispar (Figura 32F, Anexos A-79) e L. lichyi (Figura 33J, Anexos A-80) e o estado 3 em L. battistinii (com 6 espinhos) (Figura 33L, Anexos A-80)

Caráter 52. Espinho(s) na região basal da margem dorsal do parâmero

0 finos

1 semifoliáceos

2 foliáceos

Os espinhos na região basal da margem dorsal do parâmero apresentaram modificaçōes quanto ao seu aspecto. O aspecto semifoliáceo (estado 1) é apomorfia de L. alencari+ da série longipalpis presente também em L. dispar (Figura 32F, Anexos A- 
79) e em L. battistiniit da série cavernicola (Figura 33L, Anexos A-80). O aspecto foliáceo foi evidenciado em L. lichyi, autapomorfia da espécie (Figura 33J, Anexos A80).

Caráter 53. Espinho(s) na região basal da margem dorsal do parâmero:

$0 \quad$ inseridos diretamente na superficie do parâmero

1 inseridos em tubérculo discreto

2 inseridos em tubérculo colunar

A inserção dos espinhos em tubérculo discreto (estado 1) ocorre em $L$. souzalopesi e L. cruzi da série longipalpis e em L. battistinii da série cavernicola. O estado 2 ocorre em $L$. bifoliata e nas seguintes espécies da série cavernicola: $L$. bicormuta, L. forattinii e L. almerioi.

Caráter 54. Região pré-apical da margem ventral do paràmero:

0 não forma angulação com aspecto de cotovelo (ângulo saliente)

1 forma angulação com aspecto de cotovelo

A modificação no aspecto da margem ventral do parâmero, com o surgimento de uma angulação conferindo-lhe aspecto de cotovelo, foi evidenciada no ancestral de $L$ souzalopesit e de L. dispart e em $L$. renei da série cavernicola (Figuras $32 \mathrm{~F}$ e $32 \mathrm{H}$, Anexos A-79). Em Helcocyrtomyia esta modificação ocorreu em L. ayacuchesis e em $L$. munaypata+.

Caráter 55. Papilas tergais no segundo tergito abdominal: 
0 presentes

1 ausentes

Em alguns tergitos dos machos observam-se papilas tergais que aparecem, ao microscópio óptico, como pontos escuros inseridos em área central bastante clara, neste caso o tergito não apresenta cerdas (Figura 34 Anexo A-81). Estas papilas podem estar entre as cerdas e distribuídas de maneira aleatória, o que dificulta a visualização ao microscópio óptico. A presença dessas papilas desde o segundo até o sétimo tergito em Phlebotominae é considerada o estado plesiomórfico para este caráter. A ausência destas estruturas em alguns ou em todos os tergitos abdominais é a derivação observada. A ausência das papilas tergais no segundo tergito abdominal é apomorfia de Lutzomyia com a presença destas nas espécies da série castroi.

Caráter 56. Papilas tergais no terceiro tergito abdominal:

$0 \quad$ presentes

1 ausentes

Considerando-se a ausência das papilas tergais no terceiro tergito abdominal no ancestral de Lutzomyia verifica-se que em Lutzomyia s. str. houve modificação para presença de papilas em $L$. cruzi e L. longipalpis da série longipalpis, nas espécies da série castroi e em L. falcata da série cruciata. No subgênero Helcocyrtomyia a modificação, presença de papilas foi verificada em L. galatiae, em L. quillabamba, nas espécies da série caballeroi e em L. wattsi, L. gonzaloi, L. hartmanni e L. kirigtiensis da série sanguinaria.

Caráter 57. Papilas tergais no quarto tergito abdominal: 

ausentes

A ausência das papilas tergais no quarto tergito abdominal foi verificada em $L$. ischnacantha, em L. bifoliata; em L. souzalopesi; em L. dispar; em L. lichyi; na série cavernicola com reversão em: L. forattinii e L. almerioi. Em Helcocyrtomyia as espécies da série noguchii, L. pallidithorax e L. sanguinaria - não apresentam papilas.

Caráter 58. Papilas tergais no quinto tergito abdominal:

0 presentes

1 ausentes

Ausência de papilas tergais no quinto segmento abdominal foi evidenciada em L. gaminarai + da série longipalpis, em L. dispar e em L. bicornuta+ da série cavernicola com reversão em $L$. forattinii e L. almerioi. No subgênero Helcocyrtomyia a apomorfia foi observada na série noguchii+, em L. pallidithorax e L. sanguinaria ${ }^{+}$

Caráter 59. Papilas tergais no sexto tergito abdominal:

$0 \quad$ presentes

1 ausentes

As papilas tergais estão ausentes no sexto tergito abdominal de $L$. gaminarai+, L. dispar e L. cavernicolat do subgênero Lutzomyia. Em Helcocyrtomyia as papilas estão ausentes em L. blancasi+, L. pallidithorax e L. sanguinaria+.

Caráter 60. Papilas tergais no sétimo tergito abdominal: 
$0 \quad$ presentes

1 ausentes

As papilas tergais estão ausentes no sétimo tergito abdominal de $L$. ischnacantha, L. gaminarai+, de L. dispar e de L. bicormuta+ (série cavernicola). Em Helcocyrtomyia a ausência das papilas foi registrada no sétimo tergito de L. blancasit (série noguchii), L. pallidithorax, L. caballeroi e L. sanguinaria+.

Caráter 61. Corpo da espermateca:

0 vesiculoso liso

$1 \quad$ tubular segmentado

A abertura genital das fềmeas, ou câmara genital, está entre os ramos da furca genital. Nesta abertura desembocam os ductos das espermatecas e das glândulas acessórias. As espermatecas e as glândulas acessórias (secretam substância adesiva associada à postura dos ovos) são estruturas internas. As espermatecas apresentam corpo e ductos que desembocam na câmara genital como já mencionado anteriormente. Os ductos podem ser individuais, mas em alguns grupos unem-se em determinado ponto formando o ducto comum. O corpo das espermatecas pode ser saculiforme ou tubular. Quando tubular pode sofrer segmentação formando anéis que variam em sua conformação, principalmente os primeiros que podem ter o diâmetro equivalente, maior ou menor que os demais. A presença de um par de espermatecas com corpo vesiculoso, bem como a ausência de ducto comum representa o estado plesiomórfico para Phlebotominae. As espermatecas com corpo tubular segmentado estão presentes nas especies de Lutzomyia. 
Caráter 62. Diâmetro do anel apical das espermatecas :

0 equivalente ao dos que lhe antecedem

1 nitidamente maior que o dos antecedentes

$\mathrm{O}$ anel apical da espermateca pode apresentar diâmetro variável. A apomorfia representada pelo anel apical nitidamente maior que seus antecedentes (Figura 35C, Anexos A-82) foi evidenciada em $L$. ischnacantha; em L. bifoliata; em L. dispar; em $L$. lichyi; em L. amarali da série castroi; em L. falcata+; em L. bicornuta; em L. battistinii e em L. cavernicola da série cavernicola. No subgênero Helcocyrtomyia a modificação foi observada em L. peruensis; em L. tejadai; em L. ceferinoi da série caballeroi e em $L$. hartmanni e L. tortura da série sanguinaria.

Caráter 63. Diâmetro do anel apical da espermateca:

0 equivalente ao dos que lhe antecedem

1 nitidamente menor do que os que imediatamente lhe antecedem

A apomorfia representada pelo anel apical nitidamente menor que seus antecedentes foi evidenciada em L. ayacuchensis (série peruensis) e L. munaypata (série caballeroi) 


\subsection{Análise do cladograma}

A análise dos 63 caracteres observados (tabela x) resultou em 8998 cladogramas igualmente parcimoniosos $\quad(\mathrm{IC}=0,187, \quad \mathrm{IR}=0,653, \quad \mathrm{ICR}=0,122, \quad \mathrm{IH}=0,813$, comprimento=588). Para se obter um refinamento do resultado efetuou-se a pesagem sucessiva dos caracteres que resultou em 31 cladogramas igualmente parcimoniosos. $\mathrm{O}$ cladograma de consenso semi-estrito obtido (figura) apresenta os seguintes índices: $\mathrm{IC}=0,378, \mathrm{IR}=0,84, \mathrm{ICR}=0,318, \mathrm{IH}=0,622$.

No cladograma foi possivel constatar que, com a composição de espécies delineada para este estudo, o táxon denominado gênero Lutzomyia é monofilético e apresenta dois subgrupos também monofiléticos

$\mathrm{Na}$ composição do grupo mais basal contido em Lutzomyia estão as espécies que pertencem aos táxons designados por GALATI (1995) como subgêneros Lutzomyia s. str., Castromyia e Tricholateralis que não foram sustentados como tal na análise. Esta afirmação se baseia no fato de que as espécies pertencentes a estes dois últimos subgêneros, embora tenham se configurado como dois pequenos grupos monofiléticos, estão contidos em um grupo maior. O subgênero Lutzomyia s. str. com a composição proposta por GALATI, 1995 não se manteve e o que se apresentou foram dois grupos monofiléticos: $L$. bicormuta + e $L$. ischnacantha+. Este último grupo contém 3 pequenos grupos monofiléticos além de 4 espécies não agrupadas: L. ischnacantha, L. bifoliata, $L$. dispar e L. lichyi. Assim sendo considera-se a pertinência de se manter todo este conjunto como subgênero Lutzomyia s. str. composto por 4 séries de espécies e as 4 espécies não agrupadas. Caso contrário para se seguir as categorias adotadas por GALATI, 1995 seria necessário criar 4 subgêneros monoespecificos, manter os três 
subgêneros já estabelecidos (Lutzomyia s. str, Castromyia e Tricholateralis) e criar mais um para $L$. bicornutat, o que causaria mais polêmica na já tão controversa classificação dos flebotomíneos. Assim sendo, propõe-se o subgênero Lutzomyia s. str. considerandose o subgênero Castromyia como sinônimo da série castroi com a mesma composição de espécies: $L$. castroi, L. amarali e $L$. caligata; o subgênero Tricholateralise sinônimo da série cruciata com a mesma composição de espécies: L. falcata, L. cruciata, L. gomezi, L. diabolica, L. marinkellei, L. evangelistai, L. carvalhoi, L. spathotrichia, $L$. araracuarensis, L. legerae, L. flabellata e L. sherlocki. Os táxons que pertencem ao grupo designado de Lutzomyia s. str. por GALATI (1995) ficam agrupados como: série cavernicola composta por L. bicormuta, L. battistimii, L. forattinii, L. almerioi, L. cavernicola e L. renei; como série longipalpis composta por: L. souzalopesi, L. gaminarai, L. alencari, L. cruzi, L. ischyracantha, L. longipalpis e L. pseudolongipalpis e as espécies não agrupadas $L$. ischnacantha, $L$. bifoliata, $L$. dispar e $L$. lichyi

$\mathrm{Na}$ composição do grupo que constitui o outro conjunto de espécies de Lutzomyia, o subgênero Helcocyrtomyia evidenciou-se a formação de 4 subgrupos também designados como séries de espécies, além das espécies não agrupadas: $L$. pallidithorax, L. pescei, L. osornoi, L. tejadai, L. galatiae e L. quillabamba. As séries osornoi, peruensis e sanguinaria propostas por GALATI \& CÁCERES, 1994 não se sutentaram com a composição original. A série peruensis ficou constituida pelas espécies $L$. ayacuchensis e $L$. peruensis pois $L$. blancasi, $L$. chavinensis e $L$. noguchii passaram a constituir um outro grupo monofilético designado como série noguchii, série nova e as espécies $L$. pallidithorax, L. pescei, L. tejadai e $L$. galatiae estão como espécies não agrupadas. Das espécies pertencentes à série osornoi de GALATI \& 
CÁCERES 1994 ficaram agrupadas: L. caballeroi, L. castanea, L. larensis, L. ceferinoi, L. herreri, L. munaypata e L. rispaili constituindo a nova série denominada caballeroi; as espécies $L$. osornoi e $L$. quillabamba não se agruparam; $L$. strictivilla e $L$. wattsi apareceram agrupadas com o conjunto das espécies que compõe a série sanguinaria de GALATI \& CÁCERES 1994. esta série ficou portanto composta por: L. strictivilla, L. wattsi, L. gonzaloi, L. hartmanni, L. kirigetiensis, L. sanguinaraia, L. sp de Pichinde, L. cirrita, L. scorzai, L. tortura, L. guderiani, L. adamsi e L. monzonesis.

Os táxons propostos se constiutíram pelas sinapomorfias descritas a seguir, o elenco de caracteres e seus estados está em anexo(Anexos A-19- A22).

As espécies do gênero Lutzomyia foram agrupadas pelos seguintes caracteres: 1 (estado 3), 2 (estado 2), 3 (estado 2), 4 (estado 5), 6 (estado 2), 7 (estado 1), 8 (estado 1), 9 (estado 1), 10 (estado 1); 12, (estado 1), caráter 14 (estado 1), caráter 15 (estado 1), caráter 16 (estado1), caráter 17 (estado 1), caráter 18 (estado1), 20 (estado 1), 21, 22, 25 26, 27, 28 (estado 2), 2932,35 (estado 1), 40, 42 (estado 1), 46 (estado 1), 47 (estado 1), 48 (estado 2), 51 (estado 1), 55, 56, 61.

As espécies do subgênero Lutzomyia s. str. compartilham os seguintes caracteres: 34, 46 (estado 1), 50 e 51 (estado 1). As espécies da série cavernicola compartilham os seguintes caracteres: 4 (estado 3), 43 (estado 2), 44, 57, 58 e 60 .

A série longipalpis é sustentada pelas seguintes sinapomorfias: 4 (estado 1), 6 (estado 2), 19 e 29.

As espécies da série castroi compartilham os caracteres: 9 (estado 2), 25 (estado 0), 26 (estado zero), 55 (estado zero) e 56 (estado zero).

As espécies que compõem a série cruciata foram agrupadas pelos seguintes 
caracteres: 4 (estado 1), 11, 14 (estado 1),24,38,41 (estado 3) e 45 (estado 2) e 62

O subgênero Helcoyrtomyia, grupo-irmão de Lutzomyia s. str. pelas sinapomorfias representadas pelos caracteres 3 (estado 3), 4 (estado 3) em homoplasia com a série cavernicola (Lutzomyia s. str.), 7 (estado 2), 13 (estado 1), 17 (estado 2), 20 (estado 2), 33, 36 (estado 1), 42 (estado 2) e 43 (estado 1)

As espécies que compõem a série noguchii estão agrupadas pelas sinapomorfias: carater 9 (estado 2) em homoplasia com a série castroi (Lutzomyia s. str.), 34 (estado 1), 41 (estado 3) em homoplasia com a série cruciata (Lutzomyia s. str)., 46 (estado 2), 47 (estado 2), 49 (estado 1), 57. 58 e 60 em homoplasia com a série cavernicola (Lutzomyia s. str) e 59

As sinapomorfias das séries peruensis são: carater 16 (estado 2), 41 (estado 3) em homoplasia com as séries cruciata (Lutzomyia s. str) e noguchii, 46 (estado 2) em homoplasia com a série noguchii, 47 (estado zero).

As especies da série caballeroi compartilham os seguintes caracteres: 5 (estado 2), 7 (estado 1), 14 (estado 1) em homoplasia com a série cruciata (Lutzomyia s. str) e 56 (estado zero)

A série sanguinaria constituiu-se a partir das seguintes sinapomorfias: caráter 10 (estado 1), 43 (estado zero) e 49 (estado 1)

\subsection{Discussão geral}

Como ja referido as propostas de classificações para os flebotomineos têm sido baseadas em caracteres morfológicos. Estudos que identifiquem marcadores moleculares que possam também ser utilizados para propostas de classificação para o grupo são ainda 
poucos, porém têm colaborado para filogenia de alguns grupos de espécies

Assim sendo, MAZZONI e col. 2002 avaliando a evolução do gene period em flebotomineos estudaram as espécies L. intermedia, L. umbratilis, L. whitmani, $L$. migonei, L. evandroi L. dispar, L. renei, L. longipalpis e P. duboscqi. Os autores adotaram a classificação de YOUNG \& DUNCAN 1994 e, embora o gênero Lutzomyia não tenha a mesma composição de espécies que o da classificação aqui adotada, as espécies $L$. dispar. $L$. renei e $L$. longipalpis (que pertencem ao gênero em ambas as classificações) apareceram agrupadas.

Em 2004. BEATI e col. em análise filogenética para o gênero Lutzomyia baseada nas sequèncias $12 \mathrm{~S}$ e $28 \mathrm{~S}$ de DNA ribossômico estudaram as espécies $L$. ayacuchensis, L. caballeroi, L. castanea, L. gonzaloi, L. guderiani, L. munaypata, L noguchii, L. peruensis, L. pescei, L. quillabamba, L. scorzai, L. tejadai, L. battistinii, L. longipalpis, L. cortellezzi, L. fisheri, L. flaviscutellata, L. geniculata, L. maranonensis, L. migonei, L. nevesi, L. muneztovari, L. ovallesi, L. robusta, L. serrana, L. sherlocki, L. tuberculata, L. verrucarum, L. yulli, L. yulli yulli, Trichophoromyia sp. O cladograma resultante da análise de $12 \mathrm{SrDNA}$ revelou, como em nosso resultado, que o grupo de espécies analisadas pertencentes ao subgênero Helcocyrtomyia de GALATI (1995) compõem um grupo monofilético, confirmando a monofilia do subgênero determinada por GALATI (1995) e confiramda em nosso estudo. Os autores verificaram também que a partir deste marcador as séries de espécies de Helcocyrtomyia propostas por GALATI \& CÁCERES, 1994 não se sustentam com a composição proposta, como verificado também em nossa análise. Como em nosso resultado, as espécies L. ayacuchensis e $L$. peruensis formam um clado; as séries osornoi e sanguinaria compõem-se de maneira 
diferente. As espécies L. caballeroi e $L$. castanea da série ososmoi de GALATI e CÁCERES (1994) permanencem agrupadas na série caballeroi. As espécies $L$. guderiani e $L$.gonzaloi também aparecem agrupadas, ambas pertencem à série sanguinaria de GALATI 1995 e de nossa análise. As espécies L. longipalpis, $L$. sherlocki e L. battistinii formam um grupo monofilético; em nosso estudo as três espécies pertencem ao subgênero Lutzomyia s. str

Os resultados das abordagens moleculares somados aos obtidos em nossa análise revelam a importância de estudos filogenéticos para os gêneros de flebotomíneos como ferramentas para uma aproximação à real filogenia do grupo 


\subsection{Classificação do gênero Lutzomyia}

A classificação por subordinação proposta para o gênero Lutzomyia é mostrada a seguir:

Phlebotominae Rondani, 1840

Phlebotomini Rondani, 1840

Lutzomyiina Abonnenc \& Leger, 1976 Gênero-tipo: Lutzomyia França, 1924

Lutzomyia França, 1924

Espécie-tipo Lutzomyia longipalpis (Lutz \& Neiva, 1912)

(Lutzomyia) s. str.

Série cavernicola

L. bicornuta (Blancas \& Herrer, 1959)

L. battistinii (Hertig, 1943)

L. forattinii Galati, Rego, Nunes, \& Teruya, 1985

L. almerioi Galati \& Nunes, 1999

L. cavernicola (Costa Lima, 1932)

L. renei (Martins, Falcão \& Silva, 1957)

L. ischnacantha Martins, Souza \& Falcão, 1962

L. bifoliata Osorno-Mesa, Morales, Osorno \& Hoyos, 1970

Série longipalpis Barretto, 1962

L. souzalopesi Marins, Silva \& Falcão, 1970

L. gaminarai (Cordero, Vogelsang \& Cosio, 1928)

L. alencari Martins, Souza \& Falcão, 1962

L. cruzi (Mangabeira, 1938)

L. ischyracantha Martins, Falcão \& Silva, 1962

L. longipalpis (Lutz \& Neiva, 1912)

L. pseudolongipalpis Arrivilaga \& Feliciangeli, 2001 
L. dispar Martins \& Silva, 1963

L. lichyi (Floch \& Abbonenc, 1950)

Série castroi

L. castroi (Barretto \& Coutinho, 1941)

L. amarali (Barretto \& Coutinho, 1940)

L. caligata Martins, Falcão \& Silva, 1965

Série cruciata

L. falcata Young, Morale \& Ferro, 1994

L. cruciata (Coquillett, 1907)

L. gomezi (Nitzulescui, 1931)

L. diabolica (Hall, 1936)

L. marinkellei Young, 1979

L. evangelistai Martins \& Fraiha, 1971

L. carvalhoi (Damasceno, Causey \& Arouck, 1945)

L. spathorrichia Martins, Falcão \& Silva. 1963

L. araracuarensis Morales \& Minter, 1981

L. legerae Le Pont, Gantier, Hue, \& Valle, 1995

L. flabellata Martins \& Silva, 1964

L. sherlocki Martins, Silva \& Falcão, 1971

(Helcocyrtomyia) Barretto, 1962

Especie-tipo Lutzomyia (Helcocyrtomyia) peruensis (Shannon, 1929)

Série noguchii s. $\mathrm{n}$.

L. blancasi Galati \& Cáceres, 1990

L. chavimensis Pérez \& Ogozuku, 1999

L. noguchii Shannon, 1929

L. pallidithorax Galati \& Cáceres, 1994 
Série peruensis Barreto, 1962

L. ayacuchensis Cáceres \& Galati, 1988

L. peruensis (Shannon, 1929)

L. pescei (Hertig, 1943)

L. osornoi (Ristorcelli \& Van Ty, 1941)

L. tejadai Galati \& Cáceres, 1990

L. galatiae Le Pont, Martinez, Torres-Espejo \& Dujardin, 1998

L. quillabamba Ogusuku, Chevarria, Porras \& Pérez, 1999

Série caballeroi

L. caballeroi Blancas, Cáceres \& Galati, 1989

L. castanea Galati \& Cáceres, 1994

L. larensis Arredondo, 1987

L. ceferinoi (Ortiz \& Alavarez, 1963)

L. herreri Galati \& Cáceres 2003

L. munaypata Ogususku, Chevarria, Porras \& Pérez, 1999

L. rispaili Torres-Espejo, Cáceres \& Le Pont, 1995

Série sanguinaria Barreto, 1962

L. strictivilla Young, 1979

L. wattsi Férnadez, Carbajal, Astete \& Wooster, 1998

L. gonzaloi Ogusuku, Canales \& Pérez, 1997

L. hartmanni (Fairchild \& Hertig, 1957)

L. kirigetiensis Galati \& Cáceres, 1992

L. sanguinaria (Fairchild \& Hertig. 1957)

L. sp. de Pichinde Young, 1979.

L. cirrita Young \& Porter, 1974

L. scorzai (Ortiz, 1965) 
L. tortura Young \& Rogers, 1984

L. guderiani Torrez-Espejo, Cáceres \& le Pont, 1995

L. adamsi Fernandez, Galati, Carbajal, Wooster \& Watts, 1998 
CONCLUSÕES 


\section{Conclusões}

Com o resultado obtido a partir da análise dos 63 caracteres morfológicos foi possivel verificar que o gênero Lutzomyia é, de fato, monofiliético.

Nesta análise revelou-se a existência de apenas dois subgrupos monofiléticos dentro de Lutzomyia. Um deles, o mais basal, constituido pelo conjunto de espécies que compõem os subgêneros Lutzomyia s. str., Castromyia, e Tricholateralis e o outro pelo conjunto das espécies que compõem subgênero Helcocyrtomyia.

Para o subgrupo que corresponde ao subgênero Helcocyrtomyia não se verificou a sustentação da monofilia das séries osornoi, peruensis e sanguinaria e assim sendo uma outra classificação para o gênero Lutzomyia baseada no cladograma de consenso semi-estrito foi proposta. 


\section{REFERÊNCIAS BIBLIOGRÁFICAS}




\section{Referências Bibliográficas}

Abonnenc E, Leger N. Sur une classification rationnelle des Diptères Phlebotomidae. Cah ORSTOM sér Ent méd et Parasitol 1976a;14(1):69-78

Abonnenc $E$, Leger $N$. Rectificatif à la note: Sur une classification rationnelle des Diptères Phlebotomidae. Cah ORSTOM sér Ent méd et Parasitol $1976 \mathrm{~b} ; 14(1): 357$

Addis CJ. 1945. Phlebotomus (Dampfomyia) anthophorus n.sp and Phlebotomus diabolicus Hall from Texas (Diptera, Psychodidae). Journal of Parasitology; 31. $119-127,1945$

Aransay AM, Scoulica E, Tselentis Y, Ready PD. Phylogenetic relationship of phlebotomine sandflies inferred from small subunit nuclear ribosomal DNA Insect Molecular Biology; 9(2): 157-168, 2000.

Artemiev MM. A classification of the subfamily Phlebotominae Parassitologia $1991 ; 33: 69-77$

Arrivillaga JC, Feliciangeli D. Lutzomyia pseudolongipalpis. the first new species within the longipalpis (Diptera: Psychodidae: Phlebotominae) complex from La Rinconada, curarigua, Lara State, Venezuela. J Med Entomol 2001; 38 (6) : 783790.

Balard Y, Bermudez H, Dedet JP, Falcão ALM, Feliciangeli MD, Ferro C, Galati, EAB, Gomez-Landires EA, Herrero MV. Hervas D, Lambert M. Lebbe J, Morales A. Ogusuku E, Perez JE, Rangel EF, Sherlock IA, Torrez M, Vignes R. Wolff M, Young DG. Le project CIPA - CIPA GROUP. Computer-aided Identification Phlebotomine sandflies of America 2000 
Barretto MP. Novos subgêneros de Lutzomyia França, 1924 (Diptera, Psychodidae, Subfamilia Phlebotominae). Rev Inst Med Trop São Paulo 1962;4(2):91-100.

Barretto MP. Subfamílias e gêneros neotropicais da familia Psychodidae Big, 1854 (Diptera). Papéis avulsos Dep. Zool. São Paulo 1961;14:221-225.

Beati L, Cáceres AG, Lee JA, Munstermann LE. Systematic relationship among Lutzomyia sand flies (Diptera: Psychodidae) of Peru and Colombia based on the analysis of $12 \mathrm{~S}$ and $28 \mathrm{~S}$ ribosomal DNA sequences. Int Jour for Paras 34: $225-234,2004$

Blancas F, Cáceres AG, Galati EAB. Descrição de Lutzomyia caballeroi, sp n. (Diptera, Psychodidae, Phlebotominae) dos Andes Peruanos. Rev Bras Ent 1989;33:455463.

Booth DR, Ready PD, Smith DF. Evolution of multiple families of non-LTR retrotransposons in phlebotomine sandflies. Genet Res 1996;67(3):227-37.

Cáceres AG, Galati EAB. Descrição de Lutzomyia ayacuchensis sp n. (Diptera: Psychodidae, Phlebtotminae) dos Andes Peruanos. Rev Bras Ent 1988;32:55-61

Cáceres AG, Galati EAB, Pinto J, Paredes R, Reátegui R, Pérez J, Vhevarria L, Yáñez H, Zorrilla V. 2000. Psychodidae (Diptera) del Peru I: Phlebotominae em Huánuco, Pasco y Cusco, su relación con la enfermedad de Carrión y la leishmaniosis tegumentaria. Rev. Peru. biol. 7(1): 27-43

Cáceres AG, Galati EAB, Le Pont F, Velásquez C. 1995. La fauna flebotomica (Diptera: Psychodidae) de tres provincias de la Region Nor Oriental del Marañon, Peru. Rev. Soc. Bras. Med. Trop. 28(3):215-221, 1995. 
Cazorla D. A new species of Lutzomyia (Diptera: Psychodidae) from Venezuelan Andes region. Rev Bras Biol 1988;48:607-610.

Cazorla D, Añez N \& Nieves E. Descripcion de la hembra de Lutzomyia ceferinoi (Ortiz \& Álvarez, 1963) y redescripcion del macho. Mem Inst Oswaldo Cruz $1988 ; 83: 313-322$.

Coquillet DW. Discovery of blood sucking Psychodidae in America. Ent News $1907: 18: 101-102$

Cordero EH, Vogelsang EG, Cossio V. Phlebotomus gaminarai n. sp. Nueva especie de flebotomo del Uruguay. Bol Inst Clin Quirurgica, 1928;4(28/31):649-652

Costa Lima A. Sobre os phlebotomos americanos (Diptera: Psychodidae). Mem Inst Oswaldo Cruz $1932 ; 26(1): 15-70$.

Damasceno RG, Causey OR, Arouck R. Estudo sobre Flebotomus no Vale Amazonico. Parte V. Descrição de $F$. williamsi, $F$ deanei. $F$. carvalhoi, $F$ lopesi, $F$. castanheirai, F. fariasi, F. baityi e $F$. camphelli (Diptera- Psychodidae). Mem Inst Oswaldo Cruz 1945;43:1-30.

Davies CR. Llanis-Cuentas EA, Campo P. Monge J, Villaseca P, Dye C. Cutaneous leishmaniasis in the Peruvian Andes: risk factors identified from a village cohor study. Am J Trop med Hyg 1997;56(1):85-95

Depaquit J, Ferte H, Leger N, Killick-Kendrick R, Rioux JA, Killick-Kendrick M. Hanafi HA. Gobert S. Molecular systematics of the phlebotomine sandflies of the subgenus Paraphlebotomus (Diptera. Psychodidae, Phlebotomus) based on ITS2 rDNA sequences. Hypotheses of dispersion and speciation. Insect Mol Biol $2000 ; 9(3): 293-300$ 
Depaquit J, Perrotey S, Lecointre G, Tillier A, Tillier S, Ferte H, Kaltenbach M, Leger N. Molecular systematics of Phlebotominae: a pilot study. Paraphyly of the genus Phlebotomus. C R Acad Sci III. 1998;321(10):849-55.

Depaquit J, Ferte H, Leger N, Killick-Kendrick R, Rioux JA, Killick-Kendrick M, Hanafi HA, Gobert S. Molecular systematics of the phlebotomine sandflies of the subgenus Paraphlebotomus (Diptera, Psychodidae, Phlebotomus) based on ITS2 rDNA sequences. Hypotheses of dispersion and speciation. Insect Mol Biol $2000 ; 9(3): 293-300$

Dias ES, Barata RA, Fortes-Dias CL, Brazil RP, Miranda JC, Brandao Filho S, Linardi PM. Morphometric and phenetic studies of five geographical populations of Lutzomyia whitmani (Diptera: Psychodidae) in Brazil. J Med Entomol $1999 ; 36(6) ; 846-50$

Di Muccio T, Marinucci M, Frusteri L, Maroli M, Pesson B, Gramiccia M. Phylogenetic analysis of Phlebotomus species belonging to the subgenus Larroussius (Diptera, Psychodidae) by ITS2 rDNA sequences. Insect Biochem Mol Biol $2000 ; 30(5): 387-93$

Dyar HG, Nuñez-Tovar M. Notes on biting flies from Venezuela. Insec Inscit Menst $1927 ; 14: 152-155$

Dyar $\mathrm{HG}^{\circ}$ The present knowledge of the American species of Phlebotomus Rondani (Diptera, Psychodidae). Amer J Hyg 1929:10:112-124 
Esseghir S, Ready PD, Killick-Kendrick R, Ben-Ismail R. Mitochondrial haplotypes and phylogeography of Phlebotomus vectors of Leishmania major. Insect Mol Biol. 1997 Aug;6(3):211-25.

Fairchild GB, Hertig M. Notes on the Phlebotomus of the Panama (Diptera, Psychodidae) III. P. cruciatus, trinidadensis and gomezi. Ann Ent Soc Amer $1948 ; 41: 247-257$.

Fairchild GB \& Hertig M. Notes on the phlebotomus of Panama. IX. Descriptions of seven new species. Ann Ent Soc Amer 1952;45(4):505-528.

Fairchild GB, Hertig M. Notes on the Phlebotomus of Panama (Diptera, Psychodidae). XI. The male of P. cruciatus Coquilet and notes on related species. Ann Ent Soc Amer 1953:46:373-385

Fairchild GB. The relationships and classification of the Phlebotominae (Diptera, Psychodidae). Ann Ent Soc Am 1955;48:182-196

Fairchild GB, Hertig M.Notes on the Phlebotomus of Panama (Diptera, Psychodidae) XIII. The vexator group with descriptions of new species from Panama and California. Ann Ent Soc Amer 1957;50:325-334.

Fairchild GB \& Hertig M. Geographic distribution of the Phlebotomus sandflies of Central America (Diptera, Psychodidae). Ann Ent Soc Amer 1959;52:121-124.

Fairchild GB, Hertig M. Three new species of Phlebotomus from Mexico and Nicaragua (Diptera: Psychodidae). Proc Ent Soc Wash 1961;63:22-28.

Fairchild GB, Hertig M. Notes on the Phlebotomus of Panama (Diptera, Psychodidae) XVI. Description of new and little-known species from Panama and Central America. Ann Ent Soc Amer 1961;54:237-255 
Fairchild GB, Hertig M. Three new species of Phlebotomus from Mexico and Nicaragua (Diptera:Psychodidae). Proc Ent Soc Wash 1961;63 (1):22-28.

Fairchild GB. The relationships and classification of the Phlebotominae (Diptera, Psychodidae). Ann Ent Soc Am 1955;48:182-196.

Feliciangeli MD. The phlebotomine sandflies (Diptera: Psychodidae) of Venezuela. J Med Ent 1980;17:245-264

Forattini OP. Sobre a classificação da subfamília Phlebotominae nas Américas (Diptera Psychodidae). Pap Avul Zool 1971;24:93-111.

França, C. Observations sur le genre Phlebotomus. Brotéria, Ser. Zool. 1919;17:102-60.

França C, Parrot L. Introducion à l' étude systématique des Dipteres du genre Phlebotomus. Bull Soc Path exot 1920;13:695-708.

França C, Parrot L. Essai de classification des Phlebotomes. Arch Inst PasteurAfr $1921 ; 1: 279-284$

França C. Notes parasitologiques. IV. Phlebotomes. J Sci Mat Fis Nat 1924;3(17):2225 .

Galati EAB, Rego JR FA, Nunes VLB. Fauna Flebotomínica do Municipio de Corumbá, Mato Grosso do Sul, Brasil e descrição de Lutzomyia forattinii sp. n. (Diptera. Psychodidae, Phlebotominae). Revta Bras Ent 1985;29(2):261-266.

Galati EAB, Cáceres AG. Descrição de Lutzomyia blancasi sp n. e Lutzomyia tejadai sp n. (Diptera, Psychodidae, Phlebotominae) dos Andes Peruanos. Rev Bras Ent $1990 ; 34: 505-521$ 
Galati EAB, Cáceres AG. Descrição de Lutzomỵia kirigetiensis sp n. (Diptera. Psychodidae, Phlebotominae) do Peru e considerações sobre a composição do subgènero Helcocyrtomyia Barreto. Rev Bra Ent 1992:36:449-456.

Galati EAB. Cáceres AG. Descrição de Lutzomyia pallidithorax sp. n. e de Lutzomyia castanea sp. n. do Peru e análise cladística das séries do subgênero Helcocyrtomyia Barretto (Diptera, Psychodidae). Rev Bras Ent 1994:38(2):471-488.

Galati EAB Phylogenetic Systematics of Phlebotominae (Diptera-Psychodidae) with Emphasis on American Groups. Biol Dir Malariol y San Amb 1995;1:133-142

Galati EAB. Nunes VLS, Rego Jr FA, Oshiro ET. Chang MR. Estudo de flebotomíneos (Diptera:Psychodidae) em foco de Leishmaniose Visceral no Estado de Mato Grosso do Sul, Brasil Rev Saúde Pública 1997.31(4) 378-90

Galati EAB, Nunes VLS. Descrição de Lutzomyia (Lutzomyia) almerioi sp. n. (Diptera, Psychodidae) do Mato Grosso do Sul, Brasil Rev Bras Ent 1999;43:277-285

Galati EAB. Caceres AG. Descrição de três especies novas de Phlebotominae (Diptera. Psychodidae) do Departamento de Pasco. Peru. Rev Bras Ent 1999:43:293-299.

Galati EAB. Caceres AG, Zorrila V. Description of Lutzomvia chotensis. a new species of Peruvian Phlebotominae (Diptera. Psychodidae) Rev Bras Ent $2003 \cdot 47(4): 603-606$

Galati EAB, Caceres AG. Description of Lutzomvia (Helcocyrtomyia) herreri sp. nov (Diptera. Psychodidae, Phlebotominae) from the South Peruvian Andes. Rev Bras Ent 2003:47(4):607-613 
Galati EAB. Morfologia e taxonomia. Classificação de Phlebotominae. In Rangel EF, Lainson R. Flebotomíneos do Brasil 2003;23-175. Rio de Janeiro, Ed. FIOCRUZ.

Hashiguchi Y, Gomez EA. Monthly variation in natural infection of the sandfly Lutzomyia ayacuchensis with Leishmania mexicana in an endemic focus in the Ecuadorian Andes. Ann Trop Med Parasitol 1991;85(4):407-4011

Henning W. Phylogenetic Systematics. University of Illinois Press Urban; 1966.

Henning W. Insektenfossilien aus der unteren Kreide. IV. Psychodidae (Phlebotominae), mit einer Kritishen Ubersicht über das Phylogenetische System der Familie und die bisher beschriebenen Fossilien (Diptera) Sttut Beitr. Nakturkunde 1972; 241:1-67.

Henning W. Insect Phylogeny. A Wiley Interscienses Publication. John Wiley \& Sons. Printed in Great Britain at the Pritman Press, 1981

Hertig M. Notes on Peruvian sandflies with descriptions of Phlebotomus battistimii, $\mathrm{n}$. sp.. and P. pescei, n. sp. Am J Hyg 1943;37:246-355.

Ishikawa EA, Ready PD, de Souza AA, Day JC, Rangel EF, Davies CR. Shaw JJ. A mitochondrial DNA phylogeny indicates close relationships between populations of Lutzomyia whitmani (Diptera: Psychodidae, Phlebotominae) from the rainforest regions of Amazonia and northeast Brazil. Mem Inst Oswaldo Cruz $1999 ; 94(3): 339-45$ 
Lane, RP \& Ward, RD. The morfology and possible function of abdominal patches in males of two forms of the leishmaniasis-vector Lutzomyia longipalpis (Diptera: Phlebotominae). Cah ORSTOM Ent med Parasit 1984;22:245-9

Le Pont F, Gantier JC, Hue S, Valle S. Phlébotomes du Nicaragua. II - Description de Lutzomyia legerae n. sp. (Diptera: Psychodidae).Parasite 1995;2:75-79.

Lewis DJ, Young DG, Fairchild GB, Minter DM. Proposals for a stable classification of the Phlebotomine sandflies (Diptera: Psychodidae). Systematic Entomology $1977 ; 2: 319-332$.

Lins RM, Oliveira SG, Souza NA, de Queiroz RG, Justiniano SC, Ward RD, Kyriacou CP, Peixoto AA. Molecular evolution of the cacophony IVS6 region in sandflies Insect Mol Biol. 2002;11(2):117-22

Lutz A, Neiva A. Contribuição para o conhecimento de espécies do genero Phlebotomus existentes no Brasil. Mem Inst Oswaldo Cruz 1912; 4:82-85.

Mangabeira Filho O. Sôbre duas novas espécies de Phlebotomus (Diptera, Psychodidae) Mem Inst Oswaldo Cruz 1938;33(3):349

Mangabeira Filho O. $3^{\mathrm{a}}$ Contribuição ao estudo dos Flebotomus. Evandromyia n. subg (Diptera:Psychodidae). Mem Inst Oswaldo Cruz 1941:36:215-233

Mangabeira Filho, O. $11^{\circ}$. Contribuição ao estudo dos flebotomus (Diptera, Psychodidae). Phlebotomus oswaldoi Mangabeira, 1942. Mem. Inst. Oswaldo Cruz 1942;37:287-95

Martins AV, Willians P, Falcão AL. American sandflies (Diptera: Psychodidae, Phlebotominae. Rio de Janeiro. Academia Brasileira de Ciências;1978 
Martins AV, Falcão AL, Silva JE. Estudos sobre os flebótomos do estado de Minas Gerais - I. Phlebotomus renei n. sp. (Diptera, Psychodidae). Rev Bras Malariol Doenças Trop 1957;9:321-325

Martins AV, Souza MA, Falcão AL. Estudos sobre os flebótomos do Estado de Minas Gerais. IV: Descrição de duas novas espécies do subgênero Lutzomyia França, 1924 (Diptera, Psychodidae). Rev Bras Biol 1962;22:39-46

Martins AV, Falcào AL, Silva JE. Estudos sobre os flebótomos do Estado de Minas Gerais. V. Descrição do macho e da fêmea de "Lutzomyia (Lutzomyia) ischyracantha" sp. n. Rev Bras Biol 1962;22(3):227-232

Martins AV, Falcào AL, Silva JE. Notas sobre os flebótomos do Território de Roraima. com a descrição de três novas espécies (Diptera, Psychodiade) Mem Inst Oswaldo Cruz $1963 ; 23: 333-348$

Martins AV, Falcão AL, Silva JE. Notas sobre os flebótomos do Território de Roraima, com a descrição de três novas espécies (Diptera, Psychodiade). Mem Inst Oswaldo Cruz 1963:23:333-348.

Martins AV, Silva JE. Notas sobre os flebótomos do Estado do Acre. com a descrição de duas espécies novas (Diptera, Psychodidae) Rev Bras Biol 1964:24:127-138

Martins AV, Falcão AL, Silva JE. Notas sôbre os flebótomos do Território de Rondonia, com a descrição de seis espécies novas (Diptera, Psychodidae). Rev Bras Biol $1965 ; 25: 1-20$

Martins AV, Fraiha H. Lutzomyia evangelistai sp. n. nova especie de flebotomo do Estado do Pará (Diptera, Psychodidae, Phlebotominae) Rev Bras Biol $1971: 31: 361-365$ 
Martins AV, Silva JE, Falcão AL. Lutzomyia sherlocki sp. n., do Estado de Mato Grosso, Brasil (Diptera, Psychodidae, Phlebotominae). Rev Bras Biol 1971;31:415418

Martins AV, Morales-Farias EN. Sobre a distribuição geográfica dos flebotomíneos americanos (Diptera, Psychodidae, Phlebotominae). Rev Bras Biol 1972;32(3):361371

Martins AV, Falcão AL, Silva JE. Estudos sobre os flebótomos do Estado de Minas Gerais -IXI- descrição das fêmeas de Lutzomyia cipoensis, Falcão \& Silva, 1964 e de Lutzomyra ischnacantha Martins, Souza e Falcão, 1962 (Diptera, Psychodidae, Phlebotominae). Rev Bras Biol 1975;35(2):297-300

Martins AV, Falcão AL, Silva JE, Miranda R. Estudos sobre os flebotomíneos do estado de Minas Gerais. 15. Descrição do macho e da fêmea de Lutzomyia ramirezi n. sp. e da fêmea de Lutzomyia alencari Martins, Souza \& Falcão, 1962. Mem Inst Oswaldo Cruz 1982;77:417-424.

Martins AV, Godoy Jr TL, Silva JE. Nota sobre os flebótomos dos Estados do Paraná e Santa Catarina, com a redescrição da "Lutzomyia gaminarai" (Cordero, Vogelsang \& Cossio, 1928) (Diptera, Psychodidae). Rev Bras Biol 1961;21(3):309-316

Martins AV \& Silva JE. Nova espécie de flebótomo do Estado de Mato Grosso, "Lutzomyia (Lutzomyia) dispar" sp n. (Diptera, Psychodidae). Rev Bras Biol $1963: 23(3): 243-246$

Mazzoni CJ, Gomes CA, Souza NA, Queiroz RG. Justiniano SCB, Ward RD, Kyriacou CP. Peixoto AA. Molecular evolution of the period gene in sandflies. $\mathbf{J}$ Mol Evol. 2002:55(5):553-62. 
McAlpine J.F. Morfology and terminology adults. In: McAlpine JF, Peterson BV, Shewell GE, Teskey HJ, Vockeroth JR, Wood DM. Manual of Neartic Diptera. Otawa, Research Branch Agriculture Canada, Monograph 27, 1981, v. 1: 9-63.

Morales A, Minter MD. Estudio sôbre flebotomineos en Araracuara, Caquet, Colombia, S. A incluyendo la descripcion de Lutzomyia arararacuarensis (Diptera, Psychodidae). Biomedica 1981;3:94-116.

Nitzulescu V. Sur un Phlebotome nouveau du Venezuela. P. gomezi n. sp. Ann Parasit Hum Comp 1931;9:247-255.

Ortiz I, Álvarez A. Sôbre los Phlebotomus Americanos de cinco espinas con la descripcion de una nueva especie ( $P$. ceferinoi nov. sp.) de Venezuela (Diptera, Psychodidae). Rev Venez Sanid Asist Soc 1963;28:311-314.

Ortiz I. Contribucion al estudio de los flebótomos Americanos del grupo sanguinarius con la descripcion de una nueva espécies: Phlebotomus scorzai n. sp. (Diptera, Psychodidae). Acta Biol Venez 1965;5:25-39

Ortiz I. Phlebotomus erwindonaldoi sp. n., del grupo Peruensis (Diptera: Psychodidae) de Trujillo, Venezuela. Bol Dir Malariol San Amb 1978,18:205-210.

Peixoto AA, Gomes CA, de Amoretty PR. Lins RM, Meireles-Filho AC, de Souza NA, Kyriacou CP. New molecular markers for phlebotomine sand flies. Int $\mathbf{J}$ Parasitol. 2001;31(5-6):635-9.

Pimenta PFP, Secundino FCN, Blanco EEN. Interação Vetor-hospedeiro. In Rangel EF, Lainson R. Flebotomíneos do Brasil 2003;275-289. Rio de Janeiro. Ed. FIOCRUZ 
Rispail P, Leger N. Numerical taxonomy of Old World Phlebotominae (Diptera Psychodidae). 2. Restatement of classification upon subgeneric morphological characters. Mem Inst Oswaldo Cruz. 1998;93(6):787-93

Rispail P, Leger N. Numerical taxonomy of Old World Phlebotominae (Diptera: Psychodidae). 1. Considerations of morphological characters in the genus Phlebotomus Rondani \& Berte 1840. Mem Inst Oswaldo Cruz. 1998;93(6): 773 85

Ristorcelli A, Van Ty D. Phlébotomes d'une région de Colombie ou la verruga du Perou est devenue endemique depuis ou trois ans (Seconde note). Ann Parasit Hum Comp 1941;18:251-269.

Rondani, C. Sopra una espécie di insetto dittero. Memoria prima per service alla Ditterologia italiana. Parma1840: 1.

Shannon RC. Entomological investigations in connection with Carrión's disease. Amer J Trop Med Hyg 1929;19:131-148.

Shaw J, Rosa AT, Souza A, Cruz AN. Transmissão de outros agentes. Os flebotomíneos brasileiros como hospedeiros e vetores de determinadas espécies. In Rangel EF, Lainson R. Flebotomíneos do Brasil 2003;337-351. Rio de Janeiro, Ed. FIOCRUZ

Sokal RR \& Rohlf FJ. Biometry. The principles and practices of statistics in biology research. 20. ed. New York. Freemann and Company 1981;179-270.

Swofford DL. PAUP*4.0 - Win32/DOS Beta Version 10. Sunderland. 2000. Sianuer Association. 
Takaoka H, Gomez EA, Alexander JB, Hashiguchi Y. Natural infections with Leishmania promastigotes in Lutzomyia ayacuchensis (Diptera: Psychodidae) in an Andean focus of Ecuador. Am J Trop Med Hyg 1991;44(2):205-17.

Theodor O. Classification of the Old World species of the subfamily Phlebotominae (Diptera, Psychodidae). Bull Ent Res 1948;39:85-115.

Theodor O. On the classification of American Phlebotominae. J Med Ent 1965;2:17197.

Torgerson DG, Lampo M, Velázquez Y, Wood P. Genetic relationships among some species groups within the genus Lutzomyia (Diptera: Psychodidae). Amer Jour of Trop Med and Hyg, 69(\%):484-493, 2003

Torres-Espejo M. Cáceres AG, Le Pont F. Description de deux nouvelles espèces de phlébotomes du sous-genre Helcocyrtomyia du Piémont Andin Bolivien (Diptera, Psychodidae). Parasite 1995;2:157-162

Vargas L. Los Lutzomyinae como transmisores de enfermedades. Forattiniella n. subgen. y Aguayoimyia n. subgen. ambos del genero Lutzomyia (Diptera: Phlebotomidae). Medicina Rev Mex 1978;58:85-93.

Villaseca P, Padilla C, Ventura G, Samalvides F, Yañez H, Chevarria L, Ellis B, Rotz L, Leake J, Beati L. J Med Entomol 1990;27(4):701-

Young DG, Duncan MA. Guide to the identification and geographic distribution of Lutzomyia sandflies in Mexico, the West Indies, Central and South America (Diptera-Psychodidae). Mem Amer Ent Inst 1994;54:1-881. 
Young DG, Fairchild GB. Studies of Phlebotominae sandflies. Annual Report,

Gainsville: Department of Entomology and Nematology, University of Florida, 1974.

Young DG, Porter $\mathrm{CH}$. Lutzomyia cirrita n. sp. from Colombia with a new synonym in the genus (Diptera: Psychodidae, Phlebotminae). Florida Ent 1974;57:321-325.

Young DG, Rogers TE. The phlebotomine sand fly fauna (Diptera, Psychodidae) of Equador. J Med Ent 1984;21:597-611

World Health Organization. Programme for the surveillance and control of leishmaniasis 2002. In http://www.who.int/emc/diseases/leish/index.html

Williams P, Carvalho ALM. Description of the female of Lutzomyia (Lutzomyia) dispar, with a redescription of the male (Diptera: Psychodidae: Phlebotominae). J Med Ent $1979 ; 16: 325-330$

Wood DM and Borkent A. Phylogeny and classification of the Nematocera. In Mc Alpine JF, Wood DM (eds). Manual of Neartic Diptera 3. Otawa, Research Branch Agriculture Canada 1989 
ANEXOS 


\section{Material examinado e procedência}

Espécies de Phlebotominae

Lutzomyia almerioi - Lâmina com 3 machos (apenas 1 dos machos foi examinado) procedentes da caverna do sítio Três Morrinhos, interna, 28/11/1998, Bodoquena, Mato Grosso do Sul, Brasil./Lâmina com 6 machos (apenas 1 dos machos foi examinado) procedentes da caverna do sítio Três Morrinhos, interna, 28-29/09/1998, Bodoquena, Mato Grosso do Sul, Brasil./Lâmina com 7 machos (apenas 1 dos machos foi examinado) procedentes da caverna do sítio Três Morrinhos, interna, 28/11/1998, Bodoquena, Mato Grosso do Sul, Brasil./Lâmina com 12 machos (apenas 2 machos foram examinados) procedentes da caverna do sitio Três Morrinhos, interna, 28/11/1998, Bodoquena, Mato Grosso do Sul, Brasil.

Lutzomyia battistinii - Lâmina 1/25a: 1 fêmea coletada em peridomicilio, 18-19h, 18/07/2000, procedente de Magro Pampa, Distrito San Miguel. Provincia La Mar, Departamento Ayacucho, Peru./Lâmina 2/25a: 1 fêmea coletada em peridomicílio, 18-19h, 18/07/2000, procedente de Magro Pampa, Distrito San Miguel, Provincia La Mar, Departamento Ayacucho, Peru./Lâmina 3/25a: 1 fêmea coletada em peridomicílio, 18-19h, 18/07/2000, procedente de Magro Pampa, Distrito San Miguel, Provincia La Mar, Departamento Ayacucho, Peru./Lâmina 4/25a: 1 fêmea coletada em peridomicílio, 18-19h, 18/07/2000, procedente de Magro Pampa, Distrito San Miguel, Provincia La Mar, Departamento Ayacucho, Peru./Lâmina 5/25a: 1 fêmea coletada em peridomicílio, 18-19h, 18/07/2000, procedente de Magro Pampa, Distrito San Miguel, Provincia La Mar, Departamento Ayacucho, Peru./Lâmina 1/25a: 1 macho coletado em peridomicilio, 18-19h, 18/07/2000, procedente de Magro Pampa, Distrito San Miguel, Provincia La Mar, Departamento Ayacucho, Peru./Lâmina 2/25a: 1 macho coletado em peridomicílio, 18-19h, 18/07/2000, procedente de Magro Pampa, Distrito San Miguel, Provincia La Mar, Departamento Ayacucho, Peru./Lâmina 3/25a: 1 macho coletado em peridomicílio, 18-19h, 18/07/2000, procedente de Magro Pampa, Distrito San Miguel, Provincia La Mar, Departamento Ayacucho, Peru./Lâmina 4/25a: 1 macho coletado em peridomicílio, 18-19h, 18/07/2000, procedente de Magro Pampa, Distrito San Miguel, Provincia La Mar, Departamento Ayacucho, Peru./Lâmina 5/25a: 1 macho coletado em peridomicílio, 18-19h, 
18/07/2000, procedente de Magro Pampa, Distrito San Miguel, Provincia La Mar, Departamento Ayacucho, Peru.

Lutzomyia bicornuta - Lâmina 1/no. 3/64: 1 macho coletado por A. Cáceres, procedente de Huancavelica, Peru/Lâmina 2/no.3: 1 fêmea e 1 macho coletados por A. Cáceres, procedentes de Huancavelica, Peru./Lâmina 3/no.3/07: 1 fêmea e 1 macho coletados por A Cáceres, procedentes de Huancavelica, Peru./Làmina 4-4F/no.3/63: 1 fêmea e 1 macho coletados por A. Cáceres, procedentes de Huancavelica, Peru./Lâmina 5/no.3/58: 1 macho coletado por A. Caceres, procedente de Huancavelica, Peru./Lâmina 1F/no.3/67: 1 fèmea coletada por A. Cáceres, procedente de Huancavelica, Peru.Lâmina 2F/no.3/65: 1 fêmea coletada por A. Cáceres, procedente de Huancavelica, Peru./Lâmina 3F/no.3/69: 1 fêmea coletada por A. Cáceres, procedente de Huancavelica, Peru./ - Lâmina 5F/no.3: 1 fêmea coletada por A. Cáceres, procedente de Huancavelica, Peru

Lutzomyia bifoliata - 1 macho coletado e determinado por D. G Young, 19/09/1970, Antioquia, Colômbia - lâmina da coleção do Instituto René Rachou, Minas Gerais.

Lutzomyia cavernicola - Làmina no. 3031/NE no. 541: 1 macho coletado por A V. Martins, 30/01/1958, Gruta da Caveira, município Santa Luzia, Minas Gerais, Brasil - lâmina da coleção do Instituto René Rachou, Minas Gerais./Lâmina no. 3033/NE 541 - 1 macho coletado por A. V. Martins, 30/01/1958, Gruta da Caveira municipio Santa Luzia, Minas Gerais, Brasil - lâmina da coleção do Instituto René Rachou, Minas Gerais./Lâmina no. 11512/NE no. 164/59 - 1 macho e 1 fêmea capturados em 21/12/1959, casal em cópula, Maquiné, municipio de Cordisburgo, Minas Gerais, Brasil - lâmina da coleção do Instituto René Rachou, Minas Gerais./Lâmina no. 2517/NE no 496 - 1 fêmea capturada por J. E Silva em 26/11/1957, Vacaria, municipio de Jaboticatubas, Minas Gerais, Brasil - lâmina da coleção do Instituto René Rachou, Minas Gerais./Lâmina no. 2291/NE no.491 - 1 fêmealâmina da coleção do Instituto René Rachou, Minas Gerais./Lâmina no. 2317/NE no. 492 - 1 fêmea- lâmina da coleção do Instituto René Rachou, Minas Gerais.

Lutzomyia cruzi - Lâmina 1/168/no. 0168/gaveta 03: 1 macho coletado por Eliza e Jeucira, 20 - 22hs, 03/05/1991, galinheiro, Popular Nova, Corumbá, Mato Grosso do Sul, Brasil./Lâmina 2/184/no. 0184/gaveta 03: 1 macho coletado por B. T. Chaparro, em peridomicilio, Estação V, Corumbá, 22/05/1985, Mato Grosso do Sul, Brasil./ Lâmina 3: 1 macho coletado por Jeucira, Galati e Teruyo em isca humana, $18-20 \mathrm{hs}, 02 / 05 / 1991$, Cristo 
Redentor, Corumbá, Mato Grosso do Sul, Brasil./ Lâmina 4/173/no. 0173/gaveta 03: 1 macho coletado por B. T. Chaparro, em Centro, Estação VII, peridomicilio, 03/05/1985, Corumbá Mato Grosso do Sul, Brasil./ Lâmina 5: 1 macho coletado por M. Gonçalves, 05/11/1986, centro, Corumbá, Mato Grosso do Sul, Brasil./Lâmina 6/170/no. 0170/gaveta 03: I macho coletado por D. Magalhães, 01/09/1987, Cristo Redentor, Corumbá, Mato Grosso do Sul, Brasil./Lâmina 1F/200/no. 0200/gaveta 03: 1 fêmea coletada por M. Gonçalves, 13/07/1986, Bairro Guarani, Corumbá, Mato Grosso do Sul, Brasil// Lâmina 3F/148/no. 0148/gaveta 03: l fêmea coletada por M. Gonçalves, 06/03/1990. MS da SUCAN, distrito de Rio Verde, Rua Totico Medeiros 277, cod. 122/B18, Corumbá Mato Grosso do Sul, Brasil./Lâmina 4F: I fêmea coletada por D. Magalhães, 12/02/1990, MS da SUCAM, Mal Deodoro, casa 5, distrito de Rio Verde, cap. 140, cod. 122/E5, Corumbá, Mato Grosso do Sul, Brasil./Lâmina SF: 1 fêmea coletada por M. Gonçalves, 06/03/1990, MS da SUCAM, distrito de Rio Verde, Rua Totico Medeiros. 273, cod. 122/B-18. Corumbá. Mato Grosso do Sul, Brasil.

Lutzomyia dispar - Lâmina 1/no. 719/41: 1 macho coletado em parede de pedra, 11/09/1976, fazenda Jacarezinho, Camapuã, Mato Grosso, Brasil./ Lâmina 2/no. 500/42: 1 macho coletado em fenda de pedra, 02/12/1976, fazenda Jacarezinho, Camapuã, Mato Grosso, Brasil /Lâmina 1F: 1 fêmea coletada por Nunes. CDC, sob pedras, 18 - 20hs. 07/1987 Aquidauana, Mato Grosso do Sul, Brasil/Làmina 2F: 1 fêmea coletada por Galati e Nunes, sob pedras, CDC, 18 - 20 hs, 26/02/1987, mata Camisão, Aquidauana, Mato Grosso do Sul, Brasil./Lâmina 3F: 1 fêmea coletada por Nunes, CDC, 07/1987, sob pedras, Mata Camisão, Aquidauana. Mato Grosso do Sul, Brasil./Làmina 4F: 1 fêmea, coletado por Nunes. CDC. sob pedras, 18 - 20hs, 25/02/1987, Aquidauana, Mato Grosso do Sul, Brasil./Lâmina 5F: 1 fêmea coletado por Nunes, CDC, sob pedras, 18 - 20hs, 07/1987, Aquidauana, Mato Grosso do Sul, Brasil.

Lutzomyia forattinii - Lâmina 1F/300/no. 0300/gaveta 05: 1 fềmea coletado por Jeucira, Galati, Teruyo e Roberto, Gruta da Santa, 17 horas, 02/05/1991, Corumbá, Mato Grosso do Sul, Brasil./ Làmina 3F/314/no. 0314/gaveta 05: 1 fêmea coletada em Gruta. 15/09/1987. Corumbá, Mato Grosso do Sul, Brasil./Lâmina 4F/309/no. 0309/gaveta 05: 1 fềmea coletada por Rodrigues, CDC, 21/11/1986, Gruta, Lagoa do Tamengo, Corumbá, Mato Grosso do Sul, Brasil./Làmina 1/301/no. 0301/ gaveta 05: 1 macho coletado por Jeucira. Galati e 
Teruyo, por isca humana, 18 - 20 hs, 02/05/1991, Cristo Redentor, Corumbá, Mato Grosso do Sul, Brasil./Lâmina 2/308/ no. 0308/ gaveta 05: 1 macho coletado por Nunes na Gruta Estação, 1985, Corumbá, Mato Grosso do Sul, Brasil./Lâmina 3/311/ no. 0311/gaveta 05: 1 macho coletado em Gruta, 14/03/1985, Corumbá, Mato Grosso do Sul, Brasil./Lâmina 4/306/no. 0306/ gaveta 05/sintipo: 1 macho coletado por B. T. Chaparro, 1988, Gruta da Santa, Lagoa do Tamengo, Corumbá, Mato Grosso do Sul, Brasil./Lâmina 5/309/ no. 0309/gaveta 05: 1 macho, coletado por Nunes em Gruta, 23/06/1986, Corumbá, Mato Grosso do Sul, Brasil./Lâmina 310/ no. 0310/ gaveta 05: 1 macho coletado em Gruta, 05/1984, Corumbá, Mato Grosso do Sul, Brasil./Lâmina 333/ no. 0333/ gaveta 05: 1 macho, coletado por Nunes, 28/02/1985, Gruta, Corumbá, Mato Grosso do Sul, Brasil.

Lutzomyia gaminarai - Lâmina 41203/no. 1638/67/4: 1 fêmea- lâmina da coleção do Instituto René Rachou, Minas Gerais./Lâmina 41204/ no. 1638/67/9: 1 fêmea- lâmina da coleção do Instituto René Rachou, Minas Gerais/6 machos de Ponta, PR, lâminas 11212 , 41112 a 41117 da coleção do Instituto René Rachou, Minas Gerais.

Lutzomyia lichyi - Lâmina 1: 1 macho procedente de, Milagros de Montanen, Solano, Cajedes, 07/12/1982, Universidad de Carabobo, Venezuela./Lâmina 2/E no. 24: 1 macho procedente de Onota Distrito de Zamora, Venezuela - lâmina da coleção da Faculdade de Saúde Pública - USP, São Paulo./1 macho, Eno.24, setembro-72, E5011, lâmina 3134, gaveta 63, divisão 4, Onota, Distrito de Zamora, VenezuelA./fềmea, E2960, lâmina 1428, divisão 3, gaveta 29, coleção da FSP-USP/Phlebotomus vexillarius, 1164, parátipo fêmea, No. E4008, Lâmina 2260, divisão 1, gaveta 46, Riohas Cascadas La Vitoria Cevo Manamá, coleção da FSP-USP./ Phlebotomus vexillarius, parátipo macho, E4007, lâmina 2259, divisão 1, gaveta 46, Almirante Bocas, coleção da FSP-USP./5 machos da coleção do Instituto René Rachou, Minas Gerais: lâmina 45437, Magdalena, Venezuela/âmina 47727, Huanuco, Peru/lâmina 45935, Arenas, Venezuela/lâminas 44709, 44711, 44712 do Estado de Aragua; Venezuela.

Lutzomyia longipalpis - Lâmina 1: 1 macho coletado em Massapê, 1957, Ceará, Brasil./Lâmina 2: 1 macho, 01/1982, Teresina, Piauí, Brasil./Lâmina 2: 1 macho capturado em domicílio, 01/1982Teresina, Piauí, Brasil./Lâmina 3: 1 macho coletado por Barata, 02/1988, Ceará, Brasil./Lâmina sem numeração: 1 macho e 1 fềmea coletados em área urbana 17/05/1997, Araçatuba, São Paulo, Brasil./Lâmina sem numeração: 1 macho 
capturado em domicilio, 01/1982 Teresina, Piauí, Brasil/Lâmina 5/624/no. 0624/gaveta 12: 1 macho coletado por V. Atizigem, Nobu e Hermis Dall, Serra das Andorinhas, Brejo dos Padres, 19-20 hs, 1921, município de São Geraldo, Pará, Brasil/Lâmina 6/599/no. 0599/gaveta 12: 1 macho coletado por Galati e Cunha, 12/11/1985, Cássia dos Coqueiros, Bairro Carqueja, São Paulo, Brasil./Lâmina 1F/590/no. 0590/gaveta 12: 1 fêmea, capturada em domicilio, 01/1982, Teresina, Piauí, Brasil./Lâmina 2F/592/no. 0592/gaveta 12: 1 fêmea, capturada em domicilio, 01/1982, Teresina, Piauí, Brasil./Lâmina 3F/625/no. 0625/gaveta 12: 1 macho e 1 fêmea Criação IEC, 28 ${ }^{\text {a }}$ geração, 09/12/1992, Santarém, Pará, Brasil./Lâmina 4F/582/no. 0582/gaveta 12: 1 fêmea, coletado por Rabello, 1972, Bairro Arado, Salto de Pirapora, São Paulo, Brasil./Lâmina sem numeração: 1 macho, coletado por Deane, 1954, Sobral, Ceará, Brasil./Lâmina sem numeraçâo: 1 macho, coletado por Deane, 1954, Sobral, Ceará, Brasil/Lâmina sem numeração: 1 macho, coletado por Deane, 1954, Sobral, Ceará, Brasil./Lâmina sem numeração: 1 macho capturado em chiqueiro, 12/10/1998, Bodoquena, Mato Grosso do Sul, Brasil./Lâmina sem numeração: 1 macho capturado em chiqueiro, 30/08/1998, Bodoquena, Mato Grosso do Sul, Brasil./Lâmina sem numeração: 1 fêmea coletada em mata, copa da árvore, fazenda Pitangueiras, 16/11/1998, Mato Grosso do Sul, Brasil./Lâmina sem numeração: 1 macho coletado em área urbana, 23/04/1997, Araçatuba, São Paulo, Brasil/Lâmina sem numeração: 1 macho e 1 fêmea coletados em área urbana, 17/05/1996, Araçatuba, São Paulo, Brasil./Lâmina sem numeração: 1 macho e 1 fêmea coletados em área urbana, 17/05/1996, Araçatuba, São Paulo, Brasil./Lâmina sem numeração: 1 macho capturado em domicilio, 01/1982, Terezina, Piaui, Brasil./Lâmina sem numeração: 1 macho, capturado por CDC, 11/06/1995, Terezina, Piaui, Brasil.

Lutzomyia souzalopesi - Lâmina NE no. 2383/82: 1 macho coletado por J. E. Silva, 24/01/1982, Reserva Florestal do Rio Doce, Linhares, Espirito Santo, Brasil- lâmina da coleção do Instituto René Rachou, Minas Gerais./Lâmina NE no. 2383/82: 1 macho coletado por J. E. Silva, 24/01/1982, Reserva Florestal do Rio Doce, Linhares, Espírito Santo, Brasil lâmina da coleção do Instituto René Rachou, Minas Gerais./Lâmina NE no. 2377/82: 1 macho coletado por J. E. Silva, 20/01/1982, Reserva Florestal de Sooretama, Linhares, Espírito Santo, Brasil- lâmina da coleção do Instituto René Rachou, Minas Gerais./Lâmina no. 35792 C/NE no. $1350 / 65 /$ parátipo: 1 macho coletado por J. E. Silva, 18/05/1965, São 
José, municipio de Aracruz, Espirito Santo, Brasil- lâmina da coleção do Instituto René Rachou, Minas Gerais./Lâmina no. 35792D/NE no. 1350/65/parátipo: 1 fềmea coletada por J. E. Silva, 18/05/1965, São José, município de Aracruz, estado do Espírito Santo, Brasillâmina da coleção do Instituto René Rachou, Minas Gerais./ Lâmina NE no. 2384/82: 1 fêmea coletada por J. E. Silva, 18/05/1965, Reserva Florestal do Rio Doce, município de Linhares, Espírito Santo, Brasil- lâmina da coleção do Instituto René Rachou, Minas Gerais./Lâmina no. 35792E/NE no. 1350/65/parátipo: 1 fềmea coletada por J. E. Silva, 18/05/65, São José, município de Aracruz, Espirito Santo, Brasil- lâmina da coleção do Instituto René Rachou. Minas Gerais.

Lutzomyia ayacuchensis - Lâmina 2/938/no. 0938/gaveta 17: 1 macho coletado por Cáceres, em CDC. 18 - 6hs, 17/01/1995 em Pocca Baja, distrito de Pocca, Provincia de Chota, Cajamarca, Peru./Lâmina 3/934/no. 0934/gaveta 17: 1 macho coletado por Cáceres, 3:40 4:00, 15/04/1994, Milagro, distrito San Jose de Lurdes, Provincia de San Ignacio, Renon, Peru./Lâmina 4/937/no. 0937/gaveta 17: 1 macho coletado por Cáceres, CDC, 16 - 6 hs, 0910/10/1994, intradomicilio, Chontabamba, Poccha, Chota, Renon, Peru./Lâmina 5/914/no. 0914/gaveta 17: 1 macho coletado por Cáceres, 20/03/1992, CDC, domicílio, noite toda, La Loma (1200 m), San Jose de Lurdes, San Ignacio, Cajamarca, Peru./Lâmina 1/1F/895/no. 0895/gaveta 17: 1 macho e 1 fêmea coletados por Cáceres, 10/1991, Luc Mayog, Lucarras, Ayacucho, Peru./Làmina 3F/896/no. 0896/gaveta 17: 1 macho e 1 fềmea coletados por Cáceres, 06/12/1992, Equador./Lâmina 4F/899/no. 0899/gaveta 17: 1 fêmea coletada por Cáceres, 19-20, 2019/03/1992, em El Salto, San Jose de Lurdes, San Ignacio, Cajamarca, Peru./Lâmina 5F/915/no. 0915/gaveta 17: 1 fêmea, coletado por Cáceres, 20/03/1992, CDC, domicilio, noite toda, La Lona, San Jose de Lurdes, San Ignacio, Cajamarca, Peru./Lâmina 6F/914/no. 0914/gaveta 17: 1 fêmea coletada por Cáceres, em CDC, 20/03/1992, domicilio, noite toda, La Lona, San Jose de Lurdes, San Ignacio, Cajamarca, Peru./Lâmina 7F/871/no. 0871/gaveta 17: 1 fêmea coletada por Cáceres, 14/11/1987, coleta diurna, raiz de árvore, gruta de rosca e cuervo, El Milagro, San Jose de Lurdes, San Ignacio, Cajamarca, Peru Lutzomyia blancasi - Lâmina 9: 1 fêmea coletada pòr Cáceres, 28/06/1987, Punta de Huas, Campum, Pachon Bolognesi, departamento de Ancash, Peru./Lâmina 13: 1 fêmea coletada por Cáceres, data 28/061987, em dormitório, com fumaça e aspirador manual, Provincia de Bolognesi, departamento de Ancash, Peru./ Lâmina 14: 1 fêmea coletada por Cáceres, data 
23/06/1987, em dormitório, com fumaça e aspirador manual Provincia de Bolognesi, departamento de Ancash, Peru./Lâmina 15: 1 fềmea coletada por Cáceres, 23/06/1987, em dormitório, com aspirador manual e fumaça, Provincia de Bolognesi, departamento de Ancash, Peru./Lâmina 16: 1 fêmea coletada por Cáceres, 23/06/1987, em dormitório, com aspirador manual e fumaça, Provincia de Bolognesi, departamento de Ancash, Peru./Lâmina 18: 1 fêmea coletada por Cáceres, 23/06/1987, em dormitório, com aspirador manual e fumaça, Provincia de Bolognesi, departamento de Ancash, Peru./Lâmina 23: 1 fềmea coletada por Cáceres, 23/06/1987, em dormitório, com aspirador manual e fumaça, Provincia de Bolognesi, departamento de Ancash, Peru./Lâmina 24: 1 fêmea coletada por Cáceres, 23/06/1987, em dormitório, com aspirador manual e fumaça, Provincia de Bolognesi, departamento de Ancash, Peru./Lâmina 25: 1 fêmea coletada por Cáceres, 23/06/1987, em dormitório, com aspirador manual e fumaça. Provincia de Bolognesi, departamento de Ancash, Peru./Lâmina 26: 1 fêmea coletada por Cáceres, 23/06/1987, em dormitório, com aspirador manual e fumaça, Provincia de Bolognesi, departamento de Ancash, Peru./

Lutzomyia gonzaloi - 1 macho de Belen Anampiari, Junin,Peru, 23-II-99. lâmina do Laboratório de Flebotomíneos da FSP-USP.

Lutzomyia hartmanni - Lâmina 3990/2272/E40201/divisão 3/gaveta 43/parátipo: 1 fêmea coletada por Fairchild \& Hertgi, 24/04/1952, Cerro Campana - lâmina da coleção da Faculdade de Saúde Pública - USP, São Paulo/Lâmina 4004/2271/no. E4019/divisão 3/gaveta 46/parátipo: 1 fêmea coletada por Fairchild \& Hertgi, 24/04/1952, Cerro Campana - làmina da coleção da Faculdade de Saúde Pública - USP, São Paulo.

Lutzomyia kirigetiensis - 1 macho, E9079, làmına 6390, divisão 3, gaveta 128, Cusco, La Concvencion, Peru, 308 m, 15-IV-1985./1 macho, E9078, lâmina 6389, divisão 3, gaveta 128, Cusco, La Concvencion, Peru, 308 m, 15-IV-1985/1 macho, E9080, Lâmina 6391, divisão 3, gaveta 128, Cusco, La Convencion, Peru, 308 m, 15-IV-1985.

Lutzomyia munaypata - 1 macho de Junin, Peru. Distrito de Villa Rica, 2-3/XII/00./1 fêmea de Junin, Peru, Provincia de Oxapampa, Distrito Vila Rica, Rio La Sal, 18-VI-00 / 1 fêmea Junquinayo, Distrito Que Louna, Provncia La Convencion, Depto Puno.

Lutzomyia monzonensis - 1 macho de Yaucaube, Huanuco, Peru, lâmina do Laboratório de Flebotomineos da FSP-USP 
Lutzomyia noguchii - 1 fêmea, No. 1152, lâmina 1152, gaveta 023, Peligro, Peru, 14-VII91/1 macho, No. 1165, lâmina 1165, Gaveta 023, Lima, Peru,18-IV-92./1 macho e 1 fềmea, No. 1168, lâmina 1168, divisão e gaveta 023, Lima, Peru, 18-IV-92./1 macho e 1 fêmea No. 1163, lâmina 1163, gaveta 023, Lima, peru, 18-IV-92/1 fêmea No. 1164, lâmina 1164 , gaveta 023/1 fêmea No. 1170, lâmina 1170, gaveta 023, Peru./

Lutzomyia pallidithorax - Lâmina 6993/no. E10664/divisão 3/gaveta 139/parátipo: 1 macho coletado por Cáceres \& Diaz, 18:30-19hs, 20/03/1992, El Salto, S. José de Lurdes, San Ignacio, Cajamarca, Peru - lâmina da coleção da Faculdade de Saúde Pública - USP, São Paulo./Lâmina 6992/no. E10663/divisão 3/gaveta 139/parátipo: 1 macho coletado por Cáceres \& Diaz, 18:30-19hs, 20/03/1992, El Salto, S. José de Lurdes, San Ignacio, Cajamarca, Peru - lâmina da coleção da Faculdade de Saúde Pública - USP, São Paulo./Lâmina 6991/ no. E10662/divisão 3/gaveta 139/parátipo: 1 fêmea coletada por Cáceres \& Diaz, 19:00-20:30, 19/03/1992, El Salto, S. José de Lurdes, San Ignacio, Cajamarca, Peru - lâmina da coleção da Faculdade de Saúde Pública - USP, São Paulo./Lâmina 6990/no. E10661/divisão 3/gaveta 139/parátipo: 1 fêmea por Cáceres \& Diaz, 18:30-19:00, 20/03/1992, El Salto, S. José de Lurdes, San Ignacio, Cajamarca, Peru lâmina da coleção da Faculdade de Saúde Pública - USP, São Paulo.

Lutzomyia quillabamba - 1 macho coletado em Munaypata, Distr. Santana, Provincia La Convencion, Depto de Cusco, lâmina do Laboratório de Flebotomíneos da FSP-USP.

Lutzomyia peruensis - Lâmina 1: 1 macho coletado por Cáceres, 29/01/1986, Departamento de Ayacucho, Peru./Lâmina 2: 1 macho coletado por Cáceres, 28/03/1988, coleta diurna, 10hs, em Macapara, Huaillacayan, Bolognesi, Ancash, Peru./Lâmina 2: 1 macho coletado em 21/02/1988, Distrito de Patog Huaillacayan, Provincia de Bolognesi, Departamento de Ancash, Peru./Lâmina 3: 1 macho e 1 fêmea coletado por Cáceres, 24/06/1992, CDC, dormitório, 17-6hs, em Sancha, Huaráz Region, Chaven Peru./Lâmina 4: 1 macho coletado por Cáceres \& J. Escudeiro, data 17/06/1993, Shannon, peridomicilio, 19-23hs, Horacran, Distrito de Anta, Provincia de Carhuoz, Region de Chovin./Lâmina 5: 1 macho coletado por Cáceres \& J. Escudeiro, 15-16/06/1993, CDC, em dormitório, 18-6hs, Chautayog, Distrito de Independencia, Provincia de Huarez, Region de Chovin./Lâmina 6: 1 macho coletado por Cáceres, 27/03/1988, Chuipe Bajo, Distrito de Huaillacayan, Provincia de Bolognesi, Ancash, Peru./Lâmina 1F: 1 fêmea coletada por Cáceres, CDC, 16-6hs, 04/08/1994, 
intradomiciliar, Matipampa, Poccha Chota, Rennon, Peru./Lâmina 2F: 1 fêmea coletada por Cáceres, CDC, dormitório, 17-6hs 19/06/1992, Cashapampa, Punim R. Cavin, Peru.Lâmina 5F: 1 fềmea coletada por Cáceres \& J. Escudeiros, CDC, dormitório, 18-6hs, 14-15/06/1993, Chicney, Distrito de Independencia, provincia de Huarez, Region de Chavin, Peru./Lâmina 6F: 1 fềmea coletada por Cáceres, data 21/02/1988, Patog Huaillacayan, Provincia de Bolognesi, Ancash, Peru.

Lutzomyia rispaili - 1 macho, Yanatite, Peru, II-98, lâmina do Laboratório de Flebotomíneos da FSP-USP.

Lutzomyia sanguinaria - Lâmina 1: 1 fêmea coletada em 20/05/86, Ceiba Alta, Cangrejal, Acosta, San Jose, Costa Rica./Lâmina 2: 1 fềmea coletada em 27/08/85, CDC, Margarita, Sixaola, Talamanca, Limón, Costa Rica./Lâmina 3: 1 fêmea coletada em 10/10/85, CDC, Cote, Guatuso, Alajuela, Costa Rica/Lâmina 4: 1 fêmea coletada em 20/05/86, CDC, Ceiba Alta, Cangrejal, Acosta, San Jose, Costa Rica./Lâmina 1707/no. E3264/divisão 1/gaveta 35 : 1 macho coletado por H. A. Christensens, 1969, Gorgas, Panamá - lâmina da coleção da Faculdade de Saúde Pública - USP, São Paulo./Lâmina 1706/no. E3263/divisão 1/gaveta 35 : 1 macho coletado por H. A. Christensens, 1969, Gorgas, Panamá - lâmina da coleção da Faculdade de Saúde Pública - USP, São Paulo./Lâmina 1712/no. E3269/divisão 2/gaveta 35: 1 fêmea coletada por H. A. Christensens, 1969, Gorgas, Panamá - lâmina da coleção da Faculdade de Saúde Pública - USP, São Paulo./Lâmina 1713/ no. E3270//divisão 2/gaveta 35: 1 fềmea coletada por H. A. Christensens, 1969, Gorgas, Panamá - lâmina da coleção da Faculdade de Saúde Pública - USP, São Paulo.

Lutzomyia scorzai - 3 lâminas do Laboratório de Flebotomineos da FSP-USP: 2 fêmeas e 1 macho de Anantaba. Distr. Alto Inambai, Prov Sandra, Departamento de Puno, Peru/1 macho de Huancash, Mayo, Junin, Peru, 16-I-98.

Lutzomyia tejadai - Lâmina 1F: 1 fêmea, coletada por A. Cáceres, 12/1996, Departamento de Cuzco, Provincia de Quillamba, Peru./Lâmina 2F: 1 fềmea coletada por A. Cáceres, 12/1996, Departamento de Cuzco, Provincia de Quilabamba, Peru. Lâmina 3F: 1 fêmea coletada por A. Cáceres, 12/1996, Departamento de Cuzco, Provincia de Quilabamba, Peru./Lâmina 4F: 1 fêmea coletada por A. Cáceres, 12/1996, Departamento de Cuzco, Provincia de Quilabamba, Peru./Lâmina 12: 1 macho e 1 fêmea coletados por A. Cáceres, 06/11/88, Higueras, Huamico, Peru.Làmina 30: 2 machos coletados por A. Cáceres, 
06/11/88, Higueras, Huamico, Peru.Lâmina 31: 1 macho e 1 fêmea coletados por A Cáceres, 06/11/88, Higueras, Huamico, Peru./Lâmina 32: 2 machos coletados por A. Cáceres, 06/11/88, Higueras, Huamico, Peru./Lâmina 33: 2 machos coletados por A. Cáceres, 06/1 1/88, Higueras, Huamico, Peru

Lutzomyia amarali - Lâmina 1: 1 fêmea coletada em 24/02/81, Mata de Sete Lagoas, Brasil. Lâmina 2: 1 fêmea coletada na zona rural, armadilha de Shannon, 18:30-21:30 hs, 21/03/1994, Salto de Pirapora, Bairro Piraporão Velho, São Paulo, Brasil./Lâmina 3: 1 fêmea coletada por equipe da SUCEN, armadilha de Shannon, 18 - $20 \mathrm{hs,}$ 06/07/1972, Fazenda das Pedras, Indaiatuba São Paulo, Brasil./Lâmina 4: 1 fềmea coletada por equipe da SUCEN, 20-22:30 hs, 08/03/93, Bairro Colonização, Juquiá, São Paulo, Brasil./fêmea da Fazenda Pedras, Indaiatuba, SP, 6-VII-1992, det. Galati, 1993.

Lutzomyia cruciata - San Antonio, Toledo Belize/1 macho, coleção FSP-USP, VI-1980, col. Elias, det. Galati, 1991, Estrada BR 17 km 23, Manaus, AM.

Lutzomyia diabolica - 1 fêmea, Phlebotomus diabolicus, coleção da FSP - USP, 4912, Mazelos, México, Cañon de lobos entre Cuemavaca eYautebec, $4100 \mathrm{~m}$ altitude, 20-IX-55/1 macho, Phlebotomus diabolicus, E2387, lâmina 985, divisão 4, gaveta 20, México.

Lutzomyia evangelistai - 1 fềmea, Caxiuanã, col CDC luz, 16-VIII-1993, det. Galati, 1994./1 fêmea, Serra das Andorinhas, col. 6-VII-94.

Lutzomyia gomezi - Lâmina 239/no. 0239/gaveta 041: 1 fềmea coletado por Alvarenga, 11/1970, Estirão do Equador, Brasil./Lâmina 240/no. 0240/gaveta 04: 1 fêmea, Universidad de Carabobo, Carabobo, Venezuela./Lâmina 241/no. 0241/gaveta 04: 1 fêmea, Universidad de Carabobo, Carabobo, Venezuela/Lâmina 242/no. 0242/gaveta 04: 1 macho coletado por Elio Fernandez, 13/07/81, debaixo de folhas, San Estebes, Carabobo, Universidade de Carabobo, Venezuela.

Lutzomyia sherlocki - 2 lâminas com fềmea, 09-III-92, Ipixuana, Pará/1 fềmea de Loreto PERU, 27-28/X/95, lâminas do Laboratório de Flebotomineos-FSP.

Lutzomyia spathotrichia - 1 fềmea, No.1075, lâmina 1075, gaveta 022, INPA, Manaus, AM, det. RA Freitas, 1995.

Sciopemyia sordelli - machos e fêmeas de lâminas do Laboratório de Flebotomineos FSPUSP/São Paulo, Brasil 
Brumptomyia cardosoi - 2 machos provenientes de São Paulo, Cidade Jardim, X/1972 e VI/1973. Equipe Ent. SUSAN.

Brumptomyia nitzulescui - 1 macho, coletado por Rabello em São João da Boa Vista (Fazenda Santa Helena), III, 1983.

Warileya phlebotomanica - 8 fềmeas de Ayacucho, Parinocochas (Saquilhuaca, túnel de acequi, 2740m a.n.m.) coletados em XII-1985 por Cáceres/1 parátipo fêmea de Matucana (Acequia cueva)/1 macho de Chacachuaro, cueva, 2625m a.n.m.) coletado por Herrer e Cáceres/1 fềmea de Surco (cueva 2050 a.n.m.)coletada por Cáceres em VIII-1984/1 macho de Valle del Rimac coletado por Herrer, 1948.

Warileya ronundippenis - 2 machos e 3 fêmeas coletados em Cangrejal de Acosta (Ceiba Este) San Jose, X-1985.

Espécies de Bruchomyiinae

Bruchomyia almeidai - 10 machos e 10 fêmeas de Cássia dos Coqueiros, Bairro Carqueja, Paredão, SP, Brasil. XI-1985, coletado por Galati, Cunha e Madi

Nemopalpus sp - 4 machos e 4 fêmeas procedentes da Costa Rica, coletado por Zeledon.

Nemopalpus pillipes - 4 machos de Martinlândia (Santo Anastácio), SP, Brasil, coletado por Forattini VIII-1953./2 machos de Miracatu, SP, Brasil, coletado por Gomes em XI-1984/2 machos e 1 fêmea de Fazenda São Joaquim, São Roque, SP, coletado por Marassá, Moraes e Veiga. 


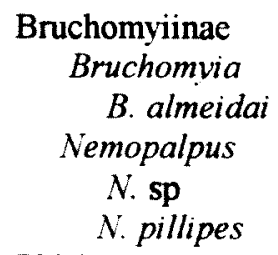

Phlebotominae Rondani, 1840

Hertigiini Abonnenc \& Leger, 1976

Hertigiina

Warileva Hertig, 1948

W. fourgassiensis Le Pont \& Desjeux, 1948

W. leponti Galati \& Cáceres. 1999

W. lumbrerasi Ogosuku, Perez, Davies \& Villaseca, 1996

W. nigrosaccula Fairchild \& Hertig. 1951

W. phlebotomanica Hertig. $1948\left(^{*}\right)$

W. rotundipennis Fairchild \& Hertig, 1951

Hertigia Fairchild, 1949

Phlebotomini Rondani. 1840

H. hertigi Fairchild, $1949\left(^{*}\right)$

Phlebotomina

Edentomyia piauiensis Galati, Andrade-Filho, Silva \& Falcão. 2003

Brumptomyiina Artemiev, 1991

Brumptomyia França \& Parrot, 1921

B. cardosoi (Barreto \& Coutinho. 1941)

B. nitizulescui (Costa Lima, 1932)

Oligodontomvia Galati, 1995

Espécie-tipo $O$. oligodonta (Young. Pérez \& Romero, 1985)

O. torensis (Le Pont, Torrez-Espejo \& Dujardin.1997)

SergentomyiinaArtemiev, 1991

Deanemyia Galati, 1995

Espécie-tipo D. samueli (Deane, 1955

Micropygomyia Barreto, 1962

(Coquilletimvia) Galati, 1995

série vexator

Lutzomyiina

Espécie-tipo M. vexator (Coquillet, 1907)

Sciopemyia Barreto, 1962

Espécie-tipo $S$. sordellii (Shannon \& Del Ponte, 1927)

S. microps (Mangabeira, 1942)

Lutzomvia França. 1924

(Helcocyrtomyia) Barreto, 1962

série sanguinaria Barreto, 1962

L. adamsi Fernadéz, Galati, Carbajal, Wooster \& Watts, 1998

L. cirrita Young \& Porter, 1974

L. guderiani Torrez-Espejo, Cáceres \& Le Pont, 1995

L. hartmanni (Fairchild \& Hertig, 1957)

L. kirigetiensis Galati \& Cáceres, 1992

L. monzonensis Ogusuku, Canales \& Pérez, 1997

L. sanguinaria (Fairchild \& Hertig. 1957)

L. scorzai (Ortiz, 1965) 
L. sp de Pichinde Young. 1979

L. tortura Young \& Rogers, 1984

série osornoi Galati \& Cáceres, 1994

L. caballeroi Blancas, Cáceres \& Galati. 1994

L. castanea Galati \& Cáceres. 1994

L. ceferinoi (Ortiz \& Alvarez. 1963)

L. herreri Galati \& Cáceres. 2003

L. larensis Arredondo, 1987

L. munaypata Ogusuku. Chevarria. Porras \& Pérez. 1999

L. osornoi (Ristorcelli \& Van Ty, 1941)

L. quillabamba Ogusuku. Chevarria. Porras \& Perez. 1999

L. rispaili Torres-Espejo, Cáceres \& Le Pont. 1995

L. strictivilla Young. 1979

L. wattsi Fernandéz, Carbajal Astete \& Wooster. 1998

série peruensis Barreto, 1962

L. avacuchensis Cáceres \& Galati. 1988

L. blancasi Galati \& Cáceres, 1990

L. chavinensis Pérez \& Ogusuku, 1999

L. galatiae Le Pont. Martinez. Torres-Espejo \& Dujardin. 1998

L. noguchii (Shanno, 1929)

L. pallidithorax Galati \& Cácers. 1994

L. peruensis (Shanno, 1929)

L. pescei (Hertig. 1943)

L. tejadai Galati \& Cáceres. 1990

(Castromvia) Mangabeira. 1942

L. amarali (Barreto \& Coutinho. 1940)

L. caligata Martins, Falcão \& Silva. 1965

L. castroi (Barreto \& Coutinho, 1941)

(Tricholateralis) Galati, 1995

L. araracuarensis Morales \& Minter. 1981

L. cararalhoi (Damasceno. Causey \& Arouck. 1945)

L. cruciata (Coquillet. 1907)

L. diabolica (Hall. 1936)

L. evangelistai Martins \& Fraiha. 1971

L. falcata Young, Morales \& Ferro, 1994

L. flabellata Martins \& Silva. 1964

L. gomezi (Nitzulescu, 1931)

L. legerae Le Pont, Gantier. Hue \& Valle. 1995

L. marinkellei Young. 1979

L. sherlocki Martins, Silva \& Falcão, 1971

L. spathotrichia Martins. Silva \& Falcão. 1963

(Lutzomvia s. str.)

L. alencari Martins, Souza \& Falcão. 1962

L. almerioi Galati \& Nunes, 1999

L. battistinii (Hertig. 1943)

L. bicornuta (Blancas \& Herrer. 1960)

L. bifoliata Osorno-Mesa. Morales. Osorno \& Hovos. 1970

L. cavernicola (Costa Lima. 1932)

L. cruzi (Mangabeira. 1938)

L. dispar Martins \& Silva. 1963

L. forattinii Galati, Rego, Nunes \& Teruya. 1985

L. gaminarai (Cordero, Vogelsang. \& Cossio, 1928

L. ischnacantha Martins, Souza \& Falcão. 1962 
L. ischyracantha Martin. Falcão \& Silva, 1962

L. lichyi (Floch \& Abonnec, 1950)

L. longipalpis (Lutz \& Neiva, 1912)

L. pseudolongipalpis Arrivilaga \& Feliciangeli, 2001

L. renei (Martins. Falcão \& Silva, 1957)

L. souzalopesi Martins, Silva \& Falcão, 1970 


\section{GLOSSÁRIO*1 $^{* 1}$}

ACCTRAN - Critério de decisão sobre a evolução de caracteres, no procedimento de otimização, em que, havendo incongruência na matriz, para cladogramas com um mesmo número de passos evolutivos, a opção com um único surgimento anterior da condição apomórfica e uma ou mais reversões para a condição plesiomórfica é preferida em relação a dois ou mais surgimentos independentes, homoplásticos da mesma apomorfia.

APOMORFIA - Estado derivado de um caráter em uma série de transformação. O conceito demanda tanto a dimensão temporal - uma condição mais recente que outra homóloga pré-existente-, quanto a dimensão material - uma condição que resultou da ocorrência de uma ou mais mutações que alteraram a forma plesiomórfica existente

APOMÓRFICA - Estado ou condição de uma estrutura que apresenta uma apomorfia

AUTAPOMORFIA - Estado ou condição de um caráter restrito a um táxon terminal, no universo abrangido por um discurso (cladograma).

AUTAPOMÓRFICO - Estado ou condição de uma estrutura que apresenta uma autapomorfia.

CARÁTER - Conceito abstrato que corresponde à mudança ocorrida em uma série de transformação com a incidência de uma ou mais mutações que alteram a forma plesiomórfica de uma estrutura para a forma apomórfica.

CLADO - Táxon monofilético 1 Baseado em e extraído de: Amorim DS. Elementos Básicos de Sistemática Filogenética. Holos
Editora \& Sociedade Brasileira de Entomologia; 1997. 
CLADOGRAMA - Dendograma em que a relação entre ramos terminais corresponde a apenas uma suposição de maior relação filogenética, indicada por apomorfias compartilhadas entre os ramos.

DENDOGRAMA - Qualquer diagrama ramificado (um sistema parcialmente ordenado) em que elementos terminais são reunidos entre si, em vários niveis, por algum critério Em sistemática, os critérios mais comuns de reunião são semelhança geral, média numérica de semelhança, e parentesco filogenético.

FILOGENIA - Em um sentido ontológico, a historia das relações filogenéticas de um grupo. Em um sentido cognitivo, o nome genérico para qualquer dendograma que expresse relações filogenéticas, seja um cladograma, uma árvore filogenética ou um cenário evolutivo.

GRUPO-EXTERNO - Toda e qualquer espécie ou grupo de espécies que filogeneticamente não pertença ao grupo supostamente monofilético abordado em um momento de uma análise.

GRUPO MONOFILÉTICO - Na definição aqui adotada corresponde ao grupo taxonômico composto por uma espécie ancestral e toda as suas espécies descendentes.

HOMOPLASIA - Relação de semelhança entre estruturas em indivíduos ou espécies distintos presentes em cada um deles devido à ocorrência independente, em níveis de generalidade distintos, de modificações que resultaram na forma final semelhante.

ÍNDICE DE CONSISTÊNCIA - Cálculo numérico que expressa a relação entre o número total de caracteres apomórficos em uma série de transformação ou em um conjunto de séries de transformação em um cladograma e o número efetivo de passos ocorridos. Esse índice sempre corresponde a um valor numérico positivo entre 0 e 1,0. 
Quando não há homoplasias esse valor é 1,0; o aumento da freqüência de homoplasias aproxima esse valor de zero.

ÍNDICE DE RETENÇÃO - Cálculo numérico que expressa a relação entre duas diferenças: a diferença entre o número efetivo de passos e o número máximo de passos possiveis e a diferença entre o número de caracteres e o número de passos possíveis. esse indice sempre corresponde a um valor numérico positivo entre 0 e 1,0 , que se aproxima de 0 á medida em que houver um maior número de autapomorfias e de homoplasias e se aproxima de 1,0 à medida em que houver um maior número de caracteres sinapomórficos não autapomórficos no cladograma e que não estiverem sujeitos a homoplasia.

ÍNDICE DE CONSISTÊNCIA REESCALONADO - produto do índice de consistência pelo indice de retenção que resulta num número positivo de 0 a 1,0 afetado mais pela ocorrência de homoplasias que pela ocorrência de autapomorfias.

MATRIZ DE CARACTERES - Base de dados que sintetiza as observações feitas sobre a condição de um conjunto limitado de caracteres para um determinado conjunto de táxons terminais. As matrizes podem ser polarizadas ou não polarizadas. Há uma função entre as matrizes polarizadas e os cladogramas correspondentes, no sentido de que a análise do cladograma deve permitir inequivocamente a recuperação da informação na matriz de caracteres.

MONOFILETISMO/MONOFILIA - Condição de um grupo que é monofilético. O termo monofilia tem sido mais amplamente utilizado.

OTIMIZAÇÃO: Procedimento de interpretação da evolução de uma série de transformação particular à luz do conjunto de caracteres disponiveis em um determinado momento da análise, de maneira que são definidos os níveis de generalidade em que 
cada condição apomórfica surgiu. Há diferentes critérios para proceder à otimização quando há incongruência entre os caracteres envolvidos.

PARCIMÔNIA - Em análise filogenética diz respeito ao critério metodológico de decisão, em que em uma análise cuja matriz de dados apresenta incongruência entre os caracteres, sobre qual, entre os cladogramas possíveis, é a reconstrução mais provável da filogenia do grupo.

PLANO-BÁSICO - Ontologicamente, como era a espécie ancestral de um táxon referido no discurso. Cognitivamente, a reconstrução dessa espécie ancestral.

PLESIOMORFIA - Estado mais antigo, pré-existente, de um caráter em uma série de transformação. O conceito demanda tanto uma dimensão temporal - uma condição que já existia antes do surgimento de outra homóloga, derivada a partir dela -, quanto uma dimensão material - uma condição que foi alterada por uma ou mais mutações que geraram a forma apomórfica correspondente.

PLESIOMÓRFICO - Estado de uma estrutura que apresenta uma plesiomorfia.

POLARIZAÇÃO (de séries de transformação) - Determinação da direção suposta para a evolução de uma série de transformação, dadas duas ou mais condições homólogas e diferentes entre si de uma estrutura, determinando qual é a plesiomófica e qual (ou quais) é a apomórfica.

REVERSÃO - Caso particular de apomorfia em que, em um passo particular de uma série de transformação, a condição apomórfica final é semelhante a uma condição plesiomórfica anterior em relação a esse passo. 
SINAPOMORFIA - Compartilhamento da condição apomórfica de um caráter por todo um conjunto de populações ou de espécies, propostas em um discurso como supostamente exclusivo delas. 


\section{Lista de caracteres}

Caráter 1. Proporção entre os comprimentos do clipeo e cabeça - macho

$$
\begin{array}{ll}
0 & 0,157-0,177 \\
1 & 0,22-0,305 \\
2 & 0,312-0,359 \\
3 & 0,363-0,396
\end{array}
$$

Caráter 2. Proporção entre os comprimentos do clipeo e cabeça - fêmea

$$
\begin{array}{ll}
0 & 0,168-0,275 \\
1 & 0,297-0,349 \\
2 & 0,35-0,4
\end{array}
$$

Caráter 3. Razão entre os comprimentos dos olhos e cabeça - macho:

$$
\begin{array}{ll}
0 & 0,694-0,713 \\
1 & 0,46-0,624 \\
2 & 0,413-0,45 \\
3 & 0,31-0,395
\end{array}
$$

Caráter 4 Razão entre os comprimentos dos olhos e cabeça - fềmea:

$$
\begin{array}{ll}
0 & 0,623-0,643 \\
1 & 0,547-0,591 \\
2 & 0,46-0,54 \\
3 & 0,299-0,45
\end{array}
$$

Caráter 5. Razão entre os comprimentos do labroepifaringe e cabeça- macho

$$
\begin{array}{ll}
0 & 0,142-0,248 \\
1 & 0,415-0,609 \\
2 & 0,623-0,711 \\
3 & 0,723-0,776 \\
4 & 0,819-0,845
\end{array}
$$

Caráter 6. Razão entre os comprimentos do labroepifaringe e cabeça - fềmea

$$
\begin{array}{ll}
0 & 0,156-0,262 \\
1 & 0,452-0,534 \\
2 & 0,589-0,815 \\
3 & 0,825-0,929 \\
4 & 0,959-0,997
\end{array}
$$

Caráter 7. Proporção entre os comprimentos do segundo palpômero e palpo - macho:

$$
\begin{array}{ll}
0 & 0,103-0,144 \\
1 & 0,149-0,186 \\
2 & 0,191-0,239
\end{array}
$$

Caráter 8. Proporção entre os comprimentos do segundo palpômero e palpo - fềmea

$$
\begin{array}{ll}
0 & 0,105-0,130 \\
1 & 0,147-0,244 \\
2 & 0,246-0,252
\end{array}
$$

Caráter 9. Proporção entre os comprimentos do terceiro palpômero e palpo - macho

$$
\begin{array}{ll}
0 & 0,148-0,173 \\
1 & 0,175-0,203 \\
2 & 0,205-0,243 \\
3 & 0,245-0,289
\end{array}
$$

Caráter 10. Proporção entre os comprimentos do terceiro palpômero e palpo - fêmea:

$$
\begin{array}{ll}
0 & 0,15-0,162 \\
1 & 0,176-0,243 \\
2 & 0,25-0,305
\end{array}
$$

Caráter 11. Proporção entre os comprimentos do quarto palpômero e palpo - macho:

$$
\begin{array}{ll}
0 & 0,155-0,203 \\
1 & 0,103-0,153
\end{array}
$$


Caráter 12. Proporção entre os comprimentos do quarto palpômero e palpo - fêmea:

$$
\begin{array}{ll}
0 & 0,171-0,207 \\
1 & 0,134-0,165 \\
2 & 0,103-0,129
\end{array}
$$

Caráter 13. Proporção entre os comprimentos do quinto palpômero e palpo - macho:

$$
\begin{array}{ll}
0 & 0,448-0,504 \\
1 & 0,356-0,444 \\
2 & 0,199-0,346
\end{array}
$$

Caráter 14. Proporção entre os comprimentos do quinto palpômero e palpo - fêmea:

$$
\begin{array}{ll}
0 & 0,451-0,501 \\
1 & 0,347-0,446 \\
2 & 0,251-0,338
\end{array}
$$

Caráter 15. Razão entre os comprimentos do segundo e quarto palpômeros - macho:

$$
\begin{array}{ll}
0 & 0,51-0,922 \\
1 & 0,962-1,237 \\
2 & 1,258-1,691 \\
3 & 1,847-2,093
\end{array}
$$

Caráter 16. Razào entre os comprimentos do segundo e quarto palpômeros - fềmea

$$
\begin{array}{ll}
0 & 0,607-0,695 \\
1 & 0,842-1,337 \\
2 & 1,355-1,791 \\
3 & 1,918-2,435
\end{array}
$$

Caráter 17. Razào entre os comprimentos do quinto e terceiro palpômeros - macho:
$\begin{array}{ll}0 & 2,853-3,288\end{array}$
l 2,226-2,584
$2 \quad 1,361-2,198$
$3 \quad 0,702-1,25$

Caráter 18. Razão entre os comprimentos do quinto e terceiro palpômeros - fêmea:

$$
\begin{array}{ll}
0 & 3,051-3,098 \\
1 & 2,0-2,634 \\
2 & 1,142-1,974 \\
3 & 0,875-1,09
\end{array}
$$

Caráter 19. Espinhos de Newstead no segundo palpômero (PII): macho e fềmea (Figura X4, Anexo)

$$
\begin{array}{ll}
0 & \text { ausentes } \\
1 & \text { presentes }
\end{array}
$$

Caráter 20. Posição dos espinhos de Newstead no terceiro palpômero (PIII) - macho e fêmea:

$$
\begin{array}{ll}
0 & \text { concentrados no terço basal } \\
1 & \text { concentrados na região mediana } \\
2 & \text { dispersos pelo segmento }
\end{array}
$$

Caráter 21. Ascóides no primeiro flagelômero (AIII) - macho e fêmea:

$$
\begin{aligned}
& 0 \text { ascóide externo implantado em } \\
& \text { posição mais basal que o interno } \\
& 1 \text { ascóide externo implantado em } \\
& \text { posição mais apical que o interno }
\end{aligned}
$$

Caráter 22. Papila peri-ascoidal em AIII - macho e fềmea:

$$
\begin{array}{ll}
0 & \text { presente } \\
1 & \text { ausente }
\end{array}
$$

Caráter 23. Papila pré-apical em AIII - macho e fềmea

$$
\begin{aligned}
& 0 \text { ausente } \\
& 1 \text { presente }
\end{aligned}
$$

Caráter 24. Papila em AV - macho e fềmea:

$$
\begin{array}{ll}
0 & \text { presente } \\
1 & \text { ausente }
\end{array}
$$

Caráter 25. Papila em AXII macho e fêmea:

$$
\begin{array}{ll}
0 & \text { presente } \\
1 & \text { ausente }
\end{array}
$$


Caráter 26. Papila em AXIII - macho e fềmea:

$$
\begin{array}{ll}
0 & \text { presente } \\
1 & \text { ausente }
\end{array}
$$

Caráter 27. Área esclerosada do cibário - fêmea:

$$
\begin{array}{ll}
0 & \text { ausente } \\
1 & \text { presente }
\end{array}
$$

Caráter 28. Armadura cibarial: dentes anteriores e posteriores - fềmea:

$$
\begin{aligned}
& 0 \text { ausentes } \\
& 1 \text { presentes os anteriores e } \\
& \text { posteriores, horizontais em relação ao } \\
& \text { lúmen da cavidade bucal } \\
& 2 \text { presentes os anteriores verticais e } \\
& \text { posteriores horizontais em relação ao lúmen }
\end{aligned}
$$

Caráter 29. Número de dentes posteriores (horizontais) no cibario - fêmea:

$$
\begin{array}{ll}
0 & \text { quatro } \\
1 & \text { mais do que quatro }
\end{array}
$$

Caráter 30. Dentes anteriores próximos e laterais à área esclerosada do cibário, mais evidentes que os demais - fêmea:

$$
\begin{array}{ll}
0 & \text { ausentes } \\
1 & \text { presentes }
\end{array}
$$

Caráter 31. Sensila ventro-cervical

$$
\begin{array}{ll}
0 & \text { presente } \\
1 & \text { ausente }
\end{array}
$$

Caráter 32. Cerdas proepimerais - macho e fêmea:

$$
\begin{array}{ll}
0 & \text { ausentes } \\
1 & \text { presentes }
\end{array}
$$

Caráter 33. Razão entre o comprimento do primeiro protarsômero e a soma dos comprimentos do segundo ao quinto - macho:

$$
\begin{aligned}
& 0 \text { menor que } 1,0 \\
& 1 \text { maior que } 1
\end{aligned}
$$

Caráter 34. Cerdas na região anterior do catepisterno - macho e fêmea:

$$
\begin{array}{ll}
0 & \text { presentes } \\
1 & \text { ausentes }
\end{array}
$$

Caráter 35. Cerda(s) pós-alar(es) - macho e fêmea:

$$
\begin{array}{ll}
0 & \text { presente } \\
1 & \text { ausente }
\end{array}
$$

Caráter 36. Extremidade inicial da nervura $R_{1}$ em relação à da $\mathrm{M}_{4}$ - macho e fềmea (Figura):

$$
\begin{array}{ll}
0 & \text { ultrapassa a de } M_{4} \\
1 & \text { coincide com a de } M_{4} \\
2 & \text { não atinge a de } M_{4}
\end{array}
$$

Caráter 37. Nervura $R_{2}$ (alfa) - machos e fềmeas

0 longa, a junção das nervuras $\mathbf{R}_{\mathbf{2}} \mathbf{R}_{\mathbf{3}}$ localiza-se aquém da extremidade apical de $\mathbf{M}_{4}$.

1 mediana, a junção de $R_{2} R_{3}$ coincide com a extremidade apical de $\mathbf{M}_{4}$ 2 reduzida, a junção $R_{2} R_{3}$ ultrapassa a extremidade apical de $\mathrm{M}_{4}$

Caráter 38. Cerdas nas pleuras abdominais:

$$
\begin{array}{ll}
0 & \text { ausentes } \\
1 & \text { presentes }
\end{array}
$$

Caráter 39. Cerda(s) na região basal da face ventral do gonocoxito:

$$
\begin{array}{ll}
0 & \text { presente(s) } \\
1 & \text { ausente }
\end{array}
$$

Caráter 40. Cerda(s) na região basal da face ventral do gonocoxito:

$$
\begin{array}{ll}
0 & \text { dispersas } \\
1 & \text { compactadas }
\end{array}
$$


Caräter 41. Número de cerdas na região basal da face ventral do gonocoxito:

$\begin{array}{ll}0 & 1-5 \\ 1 & 6 \\ 2 & 8 \\ 3 & 9-14 \\ 4 & \text { mais que } 14\end{array}$

Caráter 42 . Cerda(s) na região basal da face ventral do gonocoxito:

$$
\begin{array}{ll}
0 & \text { implantada(s) na superficie } \\
1 & \text { implantada(s) em capitulo } \\
2 & \text { implantada(s) em tubérculo }
\end{array}
$$

Caráter 43. Cerda(s) na região basal da face ventral do gonocoxito

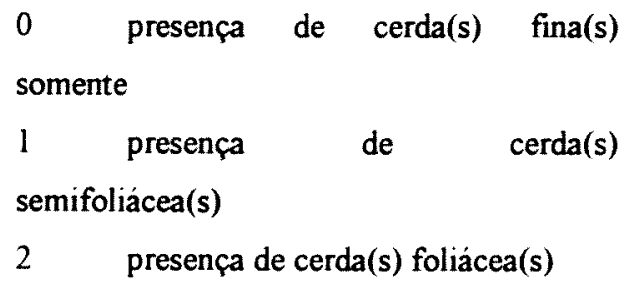

Caráter 44. Cerda(s) bem desenvolvida (s) no terço mediano da face ventral do gonocoxito:

$$
\begin{array}{ll}
0 & \text { ausente } \\
1 & \text { presente }
\end{array}
$$

Caráter 45. Espinhos apicais do gonóstilo

0 presença de dois bem
desenvolvidos
1 presença de um desenvolvido e um
atrofiado
$2 \quad$ presença de um espinho
desenvolvido

Caráter 46. Nivel de implantação do espinho intemo do gonóstilo:

$\begin{array}{ll}0 & \text { além do meio } \\ 1 & \text { no meio } \\ 2 & \text { aquém do meio }\end{array}$

Caráter 47. Nivel de implantação do espinho extemo superior do gonóstilo:

$$
\begin{array}{ll}
0 & \text { próximo ao quinto apical } \\
1 & \text { próximo ao quarto apical } \\
2 & \text { próximo ao terço apical } \\
3 & \text { no meio }
\end{array}
$$

Caráter 48. Nivel de implantação do espinho externo inferior do gonóstilo

$$
\begin{array}{ll}
0 & \text { além do meio } \\
1 & \text { no meio } \\
2 & \text { aquém do meio }
\end{array}
$$

Caráter 49. Margem dorsal do parâmero:

$$
\begin{array}{ll}
0 & \text { retilinea } \\
1 & \text { inflete-se em direção ao gonocoxito }
\end{array}
$$

Caráter 50. Espinho(s) na região basal da margem dorsal do parâmero destacado dos apicais:

$$
\begin{array}{ll}
0 & \text { ausente } \\
1 & \text { presente(s) }
\end{array}
$$

Caráter 51. Número de espinhos na região basal da margem dorsal do parâmero:

$$
\begin{array}{ll}
0 & \text { um } \\
1 & \text { dois } \\
2 & \text { très } \\
3 & \geq 4
\end{array}
$$

Caráter 52. Espinho(s) na região basal da margem dorsal do parâmero:

$\begin{array}{ll}0 & \text { finos } \\ 1 & \text { semifoliáceos } \\ 2 & \text { foliáceos }\end{array}$

Caráter 53. Espinho(s) na região basal da margem dorsal do parâmero:

0 inseridos diretamente na superficie do parâmero
1 inseridos em tubérculo discreto
2 inseridos em tubérculo colunar 
Caráter 54. Regiào pré-apical da margem ventral do parâmero:

0 não forma angulação com aspecto de cotovelo (ângulo saliente)

1 forma angulação com aspecto de cotovelo

Caráter 55. Papilas tergais no segundo tergito abdominal:

$$
\begin{array}{ll}
0 & \text { presentes } \\
1 & \text { ausentes }
\end{array}
$$

Caráter 56. Papilas tergais no terceiro tergito abdominal:

$$
\begin{array}{ll}
0 & \text { presentes } \\
1 & \text { ausentes }
\end{array}
$$

Caráter 57. Papilas tergais no quarto tergito abdominal:

$$
\begin{array}{ll}
0 & \text { presentes } \\
1 & \text { ausentes }
\end{array}
$$

Caráter 58. Papilas tergais no quinto tergito abdominal:

$$
\begin{array}{ll}
0 & \text { presentes } \\
1 & \text { ausentes }
\end{array}
$$

Caráter 59. Papilas tergais no sexto tergito abdominal:

$$
\begin{array}{ll}
0 & \text { presentes } \\
1 & \text { ausentes }
\end{array}
$$

Caráter 60. Papilas tergais no sétimo tergito abdominal:

$$
\begin{array}{ll}
0 & \text { presentes } \\
1 & \text { ausentes }
\end{array}
$$

\section{Caráter 61. Corpo da espermateca:}

$$
\begin{array}{ll}
0 & \text { vesiculoso liso } \\
1 & \text { tubular segmentado }
\end{array}
$$

Caráter 62. Diâmetro do anel apical das espermatecas:

0 equivalente ao dos que lhe antecedem

1 nitidamente maior que o dos antecedentes

Caráter 63. Diâmetro do anel apical da espermateca:
0 equivalente ao dos que lhe antecedem
1 nitidamente menor do que os que imediatamente lhe antecedem 


\section{TABELAS}


Tabela 1 - Média, limites inferior e superior do intervalo de confiança da proporção entre os comprimentos do clípeo e cabeça de machos $(\mathrm{Ccl} / \mathrm{CC})$ das espécies analisadas '.

\begin{tabular}{|c|c|c|c|}
\hline Espécies & Média & Limite inferior & Limite superior \\
\hline N. sp & 0,157 & 0,122 & 0,192 \\
\hline$N$. pilipes & 0,177 & 0,142 & 0,212 \\
\hline H. hertigi & 0,22 & 0,171 & 0,269 \\
\hline L. lichyi & 0,243 & 0,194 & 0,292 \\
\hline L. tortura & 0,27 & 0,221 & 0,319 \\
\hline L. kirigetiensis & 0,274 & 0,248 & 0,300 \\
\hline L. hartmanni & 0,279 & 0,230 & 0,328 \\
\hline L. gonzaloi & 0,287 & 0,247 & 0,327 \\
\hline L. monzonensis & 0,291 & 0,256 & 0,326 \\
\hline B. nitzulescui & 0,292 & 0,243 & 0,341 \\
\hline L. scorzai & 0,292 & 0,243 & 0,341 \\
\hline L. marinkellei & 0,294 & 0,245 & 0,343 \\
\hline D. samuellii & 0,303 & 0,263 & 0,343 \\
\hline L. souzalopesi & 0,305 & 0,279 & 0,331 \\
\hline L. guderiani & 0,312 & 0,263 & 0,361 \\
\hline L. bifoliata & 0,314 & 0,265 & 0,363 \\
\hline S. microps & 0,32 & 0,289 & 0,351 \\
\hline W. phlebotomanica & 0,32 & 0,271 & 0,369 \\
\hline L. gomezi & 0,321 & 0,272 & 0,370 \\
\hline B. almeidai & 0,322 & 0,287 & 0,357 \\
\hline L. castanea & 0,322 & 0,299 & 0,345 \\
\hline L. alencari & 0,325 & 0,285 & 0,365 \\
\hline L. longipalpis & 0,327 & 0,301 & 0,353 \\
\hline L. ceferinoi & 0,328 & 0,279 & 0,377 \\
\hline L. falcata & 0,329 & 0,280 & 0,378 \\
\hline L. pseudolongipalpis & 0,33 & 0,302 & 0,358 \\
\hline L. cruzi & 0,333 & 0,305 & 0,361 \\
\hline L. larensis & 0,333 & 0,284 & 0,382 \\
\hline L. araracuarensis & 0,336 & 0,296 & 0,376 \\
\hline L. quillabamba & 0,339 & 0,304 & 0,374 \\
\hline L. ischyracantha & 0,345 & 0,305 & 0,385 \\
\hline L. tejadai & 0,348 & 0,326 & 0,370 \\
\hline L. wattsi & 0,35 & 0,301 & 0,399 \\
\hline L. caballeroi & 0,352 & 0,317 & 0,387 \\
\hline S. sordelli & 0,358 & 0,327 & 0,389 \\
\hline L. bicormuta & 0,358 & 0,327 & 0,389 \\
\hline L. galatiae & 0,359 & 0,319 & 0,399 \\
\hline L. cavernicola & 0,363 & 0,323 & 0,403 \\
\hline L. dispar & 0,365 & 0,334 & 0,396 \\
\hline L. munaypata & 0,371 & 0,343 & 0,399 \\
\hline L. ayacuchensis & 0,372 & 0,350 & 0,394 \\
\hline L. almerioi & 0,373 & 0,354 & 0,392 \\
\hline L. ischnacantha & 0,373 & 0,345 & 0,401 \\
\hline L. peruensis & 0,373 & 0,345 & 0,401 \\
\hline L. pallidithorax & 0,375 & 0,344 & 0,406 \\
\hline L. renei & 0,38 & 0,352 & 0,408 \\
\hline L. forattinii & 0,382 & 0,354 & 0,410 \\
\hline L. battistinii & 0,389 & 0,358 & 0,420 \\
\hline E. piauiensis & 0,39 & 0,350 & 0,430 \\
\hline M. vexator & 0,39 & 0,359 & 0,421 \\
\hline L. chavinensis & 0,392 & 0,357 & 0,427 \\
\hline L. blancasi & 0,396 & 0,370 & 0,422 \\
\hline
\end{tabular}

\footnotetext{
'Nas tabelas constam somente os valores referentes às espécies cujo tamanho da amostra (n) é maior que 1.
} 
Tabela 2 - Média, limites inferior e superior do intervalo de confiança da proporção entre os comprimentos do clípeo e cabeça de fêmeas $(\mathrm{Ccl} / \mathrm{CC})$ das espécies analisadas.

\begin{tabular}{|c|c|c|c|}
\hline Espécies & Média & Limite inferior & Limite superior \\
\hline N. $\mathbf{s p}$ & 0,168 & 0,111 & 0,225 \\
\hline L. evangelistai & 0,275 & 0,249 & 0,301 \\
\hline L. amarali & 0,302 & 0,255 & 0,349 \\
\hline B. almeidai & 0,308 & 0.272 & 0,344 \\
\hline L. gomezi & 0,317 & 0,260 & 0,374 \\
\hline L. gaminarai & 0,318 & 0,277 & 0,359 \\
\hline L. ceferinoi & 0,318 & 0,261 & 0,375 \\
\hline L. castanea & 0,32 & 0.263 & 0,377 \\
\hline B. cardosoi & 0,322 & 0,275 & 0,369 \\
\hline D. samueli & 0,326 & 0,285 & 0,367 \\
\hline S. sordellii & 0,326 & 0,285 & 0,367 \\
\hline L. hartmanni & 0,328 & 0,302 & 0,354 \\
\hline L. kirigetiensis & 0,332 & 0,296 & 0,368 \\
\hline L. souzalopesi & 0,333 & 0,292 & 0,374 \\
\hline L. gonzaloi & 0,34 & 0,299 & 0,381 \\
\hline L. sanguinaria & 0,34 & 0,293 & 0,387 \\
\hline S. microps & 0,343 & 0,307 & 0,379 \\
\hline E. piauiensis & 0,345 & 0,288 & 0,402 \\
\hline L. sherlocki & 0,345 & 0,304 & 0,386 \\
\hline L. renei & 0,346 & 0,317 & 0,375 \\
\hline L. pseudolongipalpis & 0,352 & 0,305 & 0,399 \\
\hline L. alencari & 0,355 & 0,298 & 0,412 \\
\hline L. ischnacantha & 0,357 & 0,310 & 0,404 \\
\hline L. longipalpis & 0,358 & 0,329 & 0,387 \\
\hline L. pallidithorax & 0,358 & 0,301 & 0,415 \\
\hline L. dispar & 0,361 & 0,334 & 0,388 \\
\hline L. cruzi & 0,362 & 0,329 & 0,395 \\
\hline L. quillabamba & 0,362 & 0,305 & 0,419 \\
\hline W. lumbrerasi & 0,363 & 0,316 & 0,410 \\
\hline L. tejadai & 0,364 & 0,307 & 0,421 \\
\hline L. galatiae & 0,365 & 0,324 & 0,406 \\
\hline L. bicornuta & 0,366 & 0,309 & 0,423 \\
\hline L. munavpata & 0,366 & 0,319 & 0,413 \\
\hline L. ayacuchensis & 0,366 & 0,339 & 0,393 \\
\hline L. battistinit & 0,367 & 0,331 & 0,403 \\
\hline M. vexator & 0,37 & 0.323 & 0,417 \\
\hline L. almerioi & 0,37 & 0,346 & 0,394 \\
\hline L. ischyracantha & 0,373 & 0,337 & 0,409 \\
\hline L. forattinii & 0,377 & 0,346 & 0,408 \\
\hline L. cavernicola & 0,378 & 0,342 & 0,414 \\
\hline W. phlebotomanica & 0,382 & 0,341 & 0,423 \\
\hline L. caballeroi & 0,386 & 0,329 & 0,443 \\
\hline L. blancasi & 0,391 & 0,355 & 0,427 \\
\hline L. peruensis & 0,399 & 0,342 & 0,456 \\
\hline
\end{tabular}


Tabela 3 - Média, limites inferior e superior do intervalo de confiança da razào entre os comprimentos dos olhos e cabeça de machos $(\mathrm{CO} / \mathrm{CC})$ das espécies analisadas.

\begin{tabular}{|c|c|c|c|}
\hline Espécies & Média & Limite inferior & Limite superior \\
\hline L. blancasi & 0.31 & 0.257 & 0.363 \\
\hline L. peruensis & 0,38 & 0,343 & 0,417 \\
\hline L. avacuchensis & 0,392 & 0.357 & 0.427 \\
\hline W. phlebotomanica & 0.395 & 0,330 & 0,460 \\
\hline L. pallidithorax & 0.395 & 0.354 & 0.436 \\
\hline E. piauiensis & 0,413 & 0,360 & 0.466 \\
\hline M. vexator & 0,426 & 0.385 & 0.467 \\
\hline S. sordelli & 0.438 & 0,397 & 0.479 \\
\hline L. cavernicola & 0.441 & 0.388 & 0.494 \\
\hline L. bicornuta & 0.446 & 0.405 & 0.487 \\
\hline L. battistinii & 0.449 & 0,408 & 0.490 \\
\hline L. caballeroi & 0.454 & 0,389 & 0.519 \\
\hline S. microps & 0,46 & 0.419 & 0,501 \\
\hline L. tejadai & 0.462 & 0,409 & 0,515 \\
\hline L. dispar & 0.469 & 0.428 & 0.510 \\
\hline L. galatiae & 0.481 & 0.428 & 0.534 \\
\hline L. almerioi & 0,482 & 0,453 & 0.511 \\
\hline L. larensis & 0,484 & 0,419 & 0.549 \\
\hline L. forattinii & 0.487 & 0.446 & 0.528 \\
\hline H. hertigi & 0.49 & 0.425 & 0.555 \\
\hline L. ischnacantha & 0,49 & 0.453 & 0.527 \\
\hline L. renei & 0.512 & 0.475 & 0.549 \\
\hline L. castanea & 0,512 & 0.481 & 0.543 \\
\hline L. falcata & 0.516 & 0.451 & 0.581 \\
\hline L. wattsi & 0.519 & 0,454 & 0.584 \\
\hline L. longipalpis & 0,525 & 0,490 & 0.560 \\
\hline L. gomezi & 0,533 & 0.468 & 0.598 \\
\hline L. pseudolongipalpis & 0.539 & 0,474 & 0.604 \\
\hline L. lichyi & 0.54 & 0,475 & 0.605 \\
\hline L. cruzi & 0,541 & 0,504 & 0.578 \\
\hline L. ischyracantha & 0.546 & 0,493 & 0.599 \\
\hline L. adamsi & 0.554 & 0.489 & 0.619 \\
\hline B. almeidai & 0.555 & 0,509 & 0.601 \\
\hline D. samuelli & 0.564 & 0.511 & 0.617 \\
\hline L. evangelistai & 0.576 & 0.511 & 0.641 \\
\hline L. kirigetiensis & 0,584 & 0,538 & 0.630 \\
\hline B. nitzulescui & 0,586 & 0,521 & 0,651 \\
\hline L. bifoliata & 0,594 & 0,529 & 0,659 \\
\hline L. souzalopesi & 0,602 & 0.549 & 0.655 \\
\hline L. hartmanni & 0,608 & 0.543 & 0.673 \\
\hline L. guderiani & 0,624 & 0,559 & 0,689 \\
\hline$N$ pilipes & 0,697 & 0,651 & 0,743 \\
\hline$N . \mathrm{sp}$ & 0.71 & 0.664 & 0,756 \\
\hline
\end{tabular}


Tabela 4 - Média, limites inferior e superior do intervalo de confiança da razão entre os comprimentos dos olhos e cabeça de fêmeas $(\mathrm{CO} / \mathrm{CC})$ das espécies analisadas.

\begin{tabular}{|c|c|c|c|}
\hline Espócies & Média & Limite inferior & Limite superior \\
\hline L. blancasi & 0,299 & 0,230 & 0,368 \\
\hline L. peruensis & 0,332 & 0,275 & 0,389 \\
\hline L. avacuchensis & 0,364 & 0,327 & 0,401 \\
\hline W. phlebotomanica & 0,37 & 0,326 & 0,414 \\
\hline L. pallidithorax & 0,387 & 0,343 & 0,431 \\
\hline L. cavernicola & 0,389 & 0,332 & 0,446 \\
\hline L. pescei & 0,392 & 0,348 & 0,436 \\
\hline L. ceferinoi & 0,395 & 0,346 & 0.444 \\
\hline L. battistinii & 0,423 & 0,388 & 0,458 \\
\hline L. galatiae & 0,426 & 0,369 & 0,483 \\
\hline M. vexator & 0,428 & 0,379 & 0,477 \\
\hline W. lumbrerasi & 0,434 & 0,377 & 0,491 \\
\hline L. bicornuta & 0,436 & 0,367 & 0,505 \\
\hline S. microps & 0,44 & 0,383 & 0,497 \\
\hline E. piauiensis & 0,446 & 0,377 & 0,515 \\
\hline L. tejadai & 0,449 & 0,392 & 0,506 \\
\hline L. amarali & 0,449 & 0,392 & 0.506 \\
\hline S. sordellii & 0,46 & 0,416 & 0.504 \\
\hline L. forattinii & 0,464 & 0,427 & 0,501 \\
\hline L. renei & 0,467 & 0,432 & 0,502 \\
\hline L. almerioi & 0,47 & 0,440 & 0,500 \\
\hline L. castanea & 0,475 & 0,435 & 0,515 \\
\hline L. ischyracantha & 0,479 & 0,410 & 0,548 \\
\hline L. dispar & 0,479 & 0,446 & 0,512 \\
\hline L. alencari & 0,494 & 0,425 & 0,563 \\
\hline L. ischnacantha & 0,503 & 0,454 & 0.552 \\
\hline L. cruzi & 0,513 & 0,473 & 0,553 \\
\hline B. almeidai & 0,524 & 0,480 & 0,568 \\
\hline L. kirigetiensis & 0,529 & 0,460 & 0,598 \\
\hline D. samueli & 0,533 & 0,476 & 0.590 \\
\hline L. evangelistai & 0,533 & 0,464 & 0,602 \\
\hline L. sherlocki & 0,541 & 0,472 & 0,610 \\
\hline L. longipalpis & 0.547 & 0,503 & 0,591 \\
\hline L. hartmanni & 0,548 & 0,479 & 0,617 \\
\hline L. sanguinaria & 0,548 & 0,499 & 0,597 \\
\hline L. pseudolongipalpis & 0,558 & 0,489 & 0,627 \\
\hline L. gaminarai & 0,563 & 0,494 & 0,632 \\
\hline L. souzalopesi & 0,567 & 0,518 & 0,616 \\
\hline L. gomezi & 0,573 & 0,516 & 0,630 \\
\hline B. cardosoi & 0,586 & 0,517 & 0,655 \\
\hline$N$. sp. & 0.628 & 0.584 & 0.672 \\
\hline
\end{tabular}


Tabela 5 - Média, limites inferior e superior do intervalo de confiança da razào entre os comprimentos do labroepifaringe e cabeça de machos (LE/CC) das especies analisadas.

\begin{tabular}{|c|c|c|c|}
\hline Espécies & Media & Limite inferior & Limute supenor \\
\hline B. almeidai & 0.142 & 0.083 & 0.201 \\
\hline$N . \mathrm{sp}$ & 0.24 & 0,181 & 0.299 \\
\hline Vilipes & 0.248 & 0,189 & 0.307 \\
\hline II. herugi & 0,415 & 0,332 & 0.498 \\
\hline S. sordellii & 0.454 & 0,402 & 0.506 \\
\hline M. vexalor & 0.46 & 0.408 & 0.512 \\
\hline S. microps & 0.472 & 0.420 & 0.524 \\
\hline L. cruzi & 0.514 & 0.466 & 0.562 \\
\hline L. araracuarensis & 0.527 & 0.459 & 0.595 \\
\hline L. marinkellei & 0.556 & 0.473 & 0.639 \\
\hline L. longipalpis & 0.559 & 0.515 & 0.603 \\
\hline L. evangelistai & 0.56 & 0,477 & $0.6+3$ \\
\hline L. falcata & 0.568 & 0,485 & 0.651 \\
\hline L. ischnacantha & 0.569 & 0.521 & 0.617 \\
\hline B. nitzulescui & 0.583 & 0.500 & 0.666 \\
\hline L. chispar & 0.584 & 0.536 & 0.632 \\
\hline L. pseudolongipalpis & 0.589 & 0,530 & 0.648 \\
\hline L souzalopesi & 0.6 & 0.552 & 0.648 \\
\hline L. forattinii & 0.605 & 0.564 & 0.646 \\
\hline L. bicornuta & 0.609 & 0.557 & 0.661 \\
\hline L. alencart & 0.623 & 0.540 & 0.706 \\
\hline L. blancasi & 0.627 & 0.575 & 0.679 \\
\hline L. hattistinii & 0.634 & 0.582 & 0.686 \\
\hline L. rispaili & 0.642 & 0.574 & 0.710 \\
\hline L. gomezi & 0.643 & 0,560 & 0.726 \\
\hline L. ischyracantha & 0.644 & 0,576 & 0.712 \\
\hline L. hartmanni & 0.655 & 0,572 & 0.738 \\
\hline L. kirigetiensis & 0.655 & 0.611 & 0.699 \\
\hline L. almerioi & 0.663 & 0.632 & 0.694 \\
\hline L. pallidithorax & 0.665 & 0.597 & 0.733 \\
\hline L. caballeroi & 0.678 & 0.610 & $0.7+6$ \\
\hline L. chaninensis & 0.68 & 0.621 & 0.739 \\
\hline L. renei & 0.688 & 0.640 & 0.736 \\
\hline L. bifoliata & 0.699 & 0.616 & 0.782 \\
\hline L. cavernicola & 0.705 & 0.637 & 0.773 \\
\hline L. munavpata & 0.708 & 0.649 & 0.767 \\
\hline W. phlebotomanica & 0,71 & 0.627 & 0.793 \\
\hline L. castanea & 0.711 & 0.667 & 0.755 \\
\hline L. gonzaloi & 0.723 & 0.664 & 0.782 \\
\hline L. tejadai & 0,723 & 0.684 & 0.762 \\
\hline L. monzonensis & 0,736 & 0.668 & 0.804 \\
\hline L. galatiae & 0,737 & 0.689 & 0.785 \\
\hline L. quillabamba & 0.745 & 0.686 & 0.804 \\
\hline L. avacuchensis & 0.749 & 0.712 & 0.786 \\
\hline L. guderiani & 0.751 & 0.692 & 0.810 \\
\hline L. osornoi & 0.754 & 0.671 & 0.837 \\
\hline L. peruensis & 0,776 & 0.728 & 0,824 \\
\hline L. adamsi & 0.819 & 0.736 & 0,902 \\
\hline L. wattsi & 0,844 & 0,761 & 0,927 \\
\hline L. scorzai & 0.845 & 0.762 & 0.928 \\
\hline
\end{tabular}


Tabela 6 - Média, limites inferior e superior do intervalo de confiança da razão entre os comprimentos do labroepifaringe e cabeça de fêmeas (LE/CC) das espécies analisadas.

\begin{tabular}{|c|c|c|c|}
\hline Espécies & Média & Limite inferior & Limite superior \\
\hline B. almeidai & 0,156 & 0,070 & 0,242 \\
\hline$N . \mathbf{s p}$ & 0,377 & 0,291 & 0,463 \\
\hline S. sordellii & 0,452 & 0,366 & 0,538 \\
\hline L. pescei & 0,49 & 0,379 & 0,601 \\
\hline M. vexator & 0,498 & 0,402 & 0,594 \\
\hline S. microps & 0,523 & 0,412 & 0,634 \\
\hline E. piauiensis & 0,534 & 0,398 & 0,670 \\
\hline L. souzalopesi & 0,589 & 0,503 & 0,675 \\
\hline L. evangelistai & 0,604 & 0,468 & 0,740 \\
\hline L. sherlocki & 0,648 & 0,512 & 0,784 \\
\hline L. falcata & 0,656 & 0,520 & 0,792 \\
\hline L. pseudolongipalpis & 0,673 & 0,600 & 0,746 \\
\hline B. cardosoi & 0,678 & 0,542 & 0,814 \\
\hline L. longipalpis & 0,68 & 0,594 & 0,766 \\
\hline L. strictivilla & 0,694 & 0,558 & 0,830 \\
\hline L. cruzi & 0,712 & 0,633 & 0,791 \\
\hline L. ischyracantha & 0,714 & 0,603 & 0,825 \\
\hline L. amarali & 0,73 & 0,619 & 0,841 \\
\hline L. battistinii & 0,73 & 0,644 & 0,816 \\
\hline L. ischnacantha & 0,751 & 0,655 & 0,847 \\
\hline L. bicornuta & 0,753 & 0,617 & 0,889 \\
\hline L. alencari & 0,757 & 0,621 & 0,893 \\
\hline L. legerae & 0,76 & 0,649 & 0,871 \\
\hline L. pallidithorax & 0,765 & 0,686 & 0,844 \\
\hline L. tortura & 0,766 & 0,630 & 0,902 \\
\hline L. blancasi & 0,778 & 0,692 & 0,864 \\
\hline W. phlebotomanica & 0,78 & 0,694 & 0,866 \\
\hline L. forattinii & 0,794 & 0,726 & 0,862 \\
\hline L. gaminarai & 0,806 & 0,670 & 0,942 \\
\hline L. gomezi & 0,815 & 0,719 & 0,911 \\
\hline L. dispar & 0,825 & 0,761 & 0,889 \\
\hline L. sanguinaria & 0,825 & 0.729 & 0,921 \\
\hline L. kirigetiensis & 0.83 & 0,719 & 0.941 \\
\hline L. renei & 0,835 & 0,767 & 0,903 \\
\hline L. almerioi & 0,858 & 0,802 & 0,914 \\
\hline L. caballeroi & 0,86 & 0,749 & 0,971 \\
\hline L. peruensis & 0,86 & 0.774 & 0,946 \\
\hline W. lumbrerasi & 0,863 & 0,752 & 0,974 \\
\hline L. avacuchensis & 0,866 & 0,805 & 0,927 \\
\hline L. tejadai & 0,877 & 0,798 & 0,956 \\
\hline L. quillabamba & 0,884 & 0,748 & 1,020 \\
\hline L. gonzaloi & 0,905 & 0,769 & 1,041 \\
\hline L. galatiae & 0,906 & 0,827 & 0.985 \\
\hline L. castanea & 0,91 & 0,846 & 0,974 \\
\hline L. hartmanni & 0,916 & 0,805 & 1,027 \\
\hline L. cavernicola & 0,918 & 0,822 & 1,014 \\
\hline L. munavpata & 0,925 & 0,789 & 1,061 \\
\hline L. osornoi & 0,929 & 0,793 & 1,065 \\
\hline L. scorzai & 0,959 & 0,848 & 1,070 \\
\hline L. guderiani & 0,993 & 0,897 & 1,089 \\
\hline L. monzonensis & 0,997 & 0,861 & 1,133 \\
\hline
\end{tabular}


Tabela 7 - Média, limites inferior e superior do intervalo de confiança da proporção entre os comprimentos do segundo palpômero e do palpo de machos (PII/palpo) das espécies analisadas.

\begin{tabular}{|c|c|c|c|}
\hline Espécies & Média & Limite inferior & Limite superior \\
\hline B. almeidai & 0,103 & 0,028 & 0,178 \\
\hline$N . \mathbf{s p}$ & 0,12 & 0,045 & 0,195 \\
\hline E. piauiensis & 0,128 & 0,041 & 0,215 \\
\hline S. sordellii & 0,139 & 0,072 & 0,206 \\
\hline M. vexator & 0,14 & 0,073 & 0,207 \\
\hline S. microps & 0,14 & 0,073 & 0,207 \\
\hline L. ischyracantha & 0,141 & 0,054 & 0,228 \\
\hline L. cruzi & 0,143 & 0,082 & 0,204 \\
\hline L. alencari & 0,144 & 0,038 & 0,250 \\
\hline L. renei & 0,149 & 0,088 & 0,210 \\
\hline L. bicormata & 0,151 & 0,084 & 0,218 \\
\hline L. longipalpis & 0,157 & 0,110 & 0,204 \\
\hline L. pseudolongipalpis & 0,157 & 0,082 & 0,232 \\
\hline L. forattinii & 0,163 & 0,110 & 0,216 \\
\hline L. lichyi & 0,164 & 0,058 & 0,270 \\
\hline L. bifoliata & 0,165 & 0,059 & 0,271 \\
\hline L. ischnacantha & 0,166 & 0,099 & 0,233 \\
\hline L. caballeroi & 0,169 & 0,082 & 0,256 \\
\hline L. almerioi & 0,17 & 0,123 & 0,217 \\
\hline L. cavernicola & 0,17 & 0,103 & 0,237 \\
\hline L. battistinii & 0,171 & 0,114 & 0,228 \\
\hline L. noguchii & 0,171 & 0,096 & 0,246 \\
\hline L. dispar & 0,173 & 0,112 & 0,234 \\
\hline L. scorzai & 0,176 & 0,070 & 0,282 \\
\hline L. souzalopesi & 0,178 & 0,121 & 0,235 \\
\hline O. toroensis & 0,181 & 0,094 & 0,268 \\
\hline L. gaminarai & 0,184 & 0,078 & 0,290 \\
\hline L. blancasi & 0,185 & 0,128 & 0,242 \\
\hline L. castanea & 0,186 & 0,129 & 0,243 \\
\hline L. cruciata & 0,191 & 0,130 & 0,252 \\
\hline L. gomezi & 0,191 & 0,085 & 0,297 \\
\hline L. ayacuchensis & 0,192 & 0,145 & 0,239 \\
\hline L. pallidithorax & 0,193 & 0,126 & 0,260 \\
\hline L. galatiae & 0,197 & 0,136 & 0,258 \\
\hline L. munaypata & 0,197 & 0,122 & 0,272 \\
\hline L. gonzaloi & 0,198 & 0,123 & 0,273 \\
\hline L. wattsi & 0,199 & 0,093 & 0,305 \\
\hline L. peruensis & 0,2 & 0,158 & 0,242 \\
\hline L. chavinensis & 0,202 & 0,127 & 0,277 \\
\hline L. falcata & 0,203 & 0,097 & 0,309 \\
\hline L. rispaili & 0,203 & 0,116 & 0,290 \\
\hline L. tejadai & 0,203 & 0,156 & 0,250 \\
\hline L. guderiami & 0,208 & 0,133 & 0,283 \\
\hline L. castroi & 0,211 & 0,105 & 0,317 \\
\hline L.kirigetiensis & 0,211 & 0,154 & 0,268 \\
\hline L. monzonensis & 0,214 & 0,139 & 0,289 \\
\hline W. phlebotomanica & 0,215 & 0,109 & 0,321 \\
\hline L. legerae & 0,221 & 0,134 & 0,308 \\
\hline L. quillabamba & 0,224 & 0,149 & 0,299 \\
\hline H. hertigi & 0,239 & 0,133 & 0,345 \\
\hline$N$. pilipes & 0,305 & 0,230 & 0,380 \\
\hline
\end{tabular}


Tabela 8 - Média, limites inferior e superior do intervalo de confiança da proporção entre os comprimentos do segundo palpômero e do palpo de fềmeas ( $\mathrm{PII} / \mathrm{palpo}$ ) das espécies analisadas.

\begin{tabular}{|c|c|c|c|}
\hline Espécies & Média & Limite inferior & Limite superior \\
\hline B. almeidai & 0.105 & 0.089 & 0.121 \\
\hline N. sp & 0,122 & 0,107 & 0.137 \\
\hline E. piauiensis & 0,13 & 0.111 & 0.149 \\
\hline 11. vexator & 0.147 & 0.131 & 0.163 \\
\hline S. microps & 0.147 & 0.128 & 0.166 \\
\hline 1. alencari & 0,148 & 0.125 & 0.171 \\
\hline S. sordellii & 0.151 & 0.136 & 0.166 \\
\hline 1. ischyracantha & 0.156 & 0,137 & 0.175 \\
\hline L. sherlocki & 0.156 & 0.133 & 0.179 \\
\hline L. longipalpis & 0.16 & 0.150 & 0.170 \\
\hline L. batristinii & 0.163 & 0.151 & 0.175 \\
\hline L. cruzi & 0.163 & 0.150 & 0.176 \\
\hline L. falcata & 0.164 & 0.141 & 0.187 \\
\hline L. gomezi & 0,165 & 0.146 & 0.184 \\
\hline L. lichyi & 0.166 & 0.147 & 0.185 \\
\hline L. cruciata & 0.169 & 0.156 & 0.182 \\
\hline D. samueli & 0.171 & 0.152 & 0.190 \\
\hline L. souzalopesi & 0.171 & 0.155 & 0.187 \\
\hline L. bicomuta & 0.172 & 0.160 & 0.184 \\
\hline L. ischnacantha & 0.173 & 0.157 & 0.189 \\
\hline O. toroensis & 0.177 & 0.158 & 0.196 \\
\hline 1. dispar & 0.178 & 0.168 & 0.188 \\
\hline L. cavernicola & 0.179 & 0.164 & 0.194 \\
\hline L. pallidithorax & 0.179 & 0.164 & 0.194 \\
\hline L. renei & 0.183 & 0.171 & 0.195 \\
\hline L. gaminarai & 0.185 & 0,162 & 0.208 \\
\hline L. noguchii & 0.185 & 0.166 & 0.204 \\
\hline L. forattinii & 0.186 & 0.175 & 0.197 \\
\hline L. pseudolongipalpis & 0.186 & 0.174 & 0.198 \\
\hline L. peruensis & 0.186 & 0,176 & 0.196 \\
\hline L. amarali & 0,187 & 0,172 & 0.202 \\
\hline L. strictivilla & 0.192 & 0.169 & 0.215 \\
\hline L. almerioi & 0.193 & 0.183 & 0.203 \\
\hline 1. blancasi & 0.194 & 0.178 & 0.210 \\
\hline L. tejadai & 0.198 & 0.183 & 0.213 \\
\hline L. legerae & 0.199 & 0.180 & 0. 218 \\
\hline L. caballeroi & 0.202 & 0.191 & 0.213 \\
\hline L. avacuchensis & 0.204 & 0.194 & 0.214 \\
\hline II. phlebotomanica & 0.225 & 0.209 & 0.241 \\
\hline L. ceferinoi & 0.228 & 0.212 & 0.244 \\
\hline L. pescei & 0.23 & 0.211 & 0.249 \\
\hline L. tortura & 0.231 & 0.208 & 0.254 \\
\hline L. sanguinaria & 0.232 & 0.219 & 0.245 \\
\hline L. cirrita & 0.234 & 0.215 & 0.253 \\
\hline L. galatiae & 0.236 & 0,223 & 0.249 \\
\hline L. gonzaloi & 0.238 & 0,223 & 0.253 \\
\hline L. sp de Pichinde & 0.238 & 0,215 & 0.261 \\
\hline W. lumbrerasi & 0,239 & 0,220 & 0.258 \\
\hline L. scorzai & 0.242 & 0.223 & 0.261 \\
\hline L. hartmanni & 0,244 & 0,225 & 0.263 \\
\hline L. castanea & 0.246 & 0.235 & 0.257 \\
\hline L. munavpata & 0.2475 & 0,231 & 0.264 \\
\hline L. adamsi & 0.248 & 0.225 & 0.271 \\
\hline L. monzonensis & 0.25 & 0,235 & 0.265 \\
\hline L. quillabamba & 0.251 & 0.235 & 0.267 \\
\hline L. guderiani & 0.252 & 0.236 & 0.268 \\
\hline
\end{tabular}


Tabela 9 - Média, limites inferior e superior do intervalo de confiança da proporção entre os comprimentos do terceiro palpômero e do palpo de machos (PIII/palpo das espécies analisadas.

\begin{tabular}{lccc}
\hline Espécies & Média & Limite inferior & Limite superior \\
\hline N. pilipes & 0,148 & 0,130 & 0,166 \\
B. almeidai & 0,173 & 0,155 & 0,191 \\
W. phlebotomanica & 0,175 & 0,149 & 0,201 \\
L. renei & 0,177 & 0,162 & 0,192 \\
L. ischyracantha & 0,182 & 0,161 & 0,203 \\
N.emopalpus sp & 0,183 & 0,165 & 0,201 \\
L. alencari & 0,184 & 0,158 & 0,210 \\
L. cruzi & 0,187 & 0,172 & 0,202 \\
L. ischnacantha & 0,187 & 0,171 & 0,203 \\
E. piauiensis & 0,189 & 0,168 & 0,210 \\
L. cavernicola & 0,191 & 0,175 & 0,207 \\
L. longipalpis & 0,191 & 0,179 & 0,203 \\
L. cruciata & 0,191 & 0,176 & 0,206 \\
L. gomezi & 0,191 & 0,165 & 0,217 \\
L. almerioi & 0,193 & 0,181 & 0,205 \\
L. pseudolongipalpis & 0,193 & 0,175 & 0,211 \\
L. pallidithorax & 0,193 & 0,177 & 0,209 \\
L.bicornuta & 0,196 & 0,180 & 0,212 \\
L. dispar & 0,196 & 0,181 & 0,211 \\
L. lichyi & 0,196 & 0,170 & 0,222 \\
L. cyacuchensis & 0,196 & 0,184 & 0,208 \\
L. forattinii & 0,197 & 0,184 & 0,210 \\
L. peruensis & 0,2 & 0,190 & 0,210 \\
M. vexator & 0,202 & 0,186 & 0,218 \\
L. tejadai & 0,203 & 0,191 & 0,215 \\
L. falcata & 0,203 & 0,177 & 0,229 \\
L. noguchii & 0,205 & 0,187 & 0,223 \\
L. galatiae & 0,206 & 0,191 & 0,221 \\
L. chavinensis & 0,21 & 0,192 & 0,228 \\
L. castroi & 0,211 & 0,185 & 0,237 \\
L. battistinii & 0,214 & 0,200 & 0,228 \\
L. souzalopesi & 0,214 & 0,200 & 0,228 \\
L. caballeroi & 0,216 & 0,195 & 0,237 \\
L. munaypata & 0,216 & 0,198 & 0,234 \\
L. blancasi & 0,217 & 0,203 & 0,231 \\
O. toroensis & 0,219 & 0,198 & 0,240 \\
L. gaminarai & 0,219 & 0,193 & 0,245 \\
L. castanea & 0,229 & 0,215 & 0,243 \\
L. bifoliata & 0,232 & 0,206 & 0,258 \\
L. legerae & 0,232 & 0,211 & 0,253 \\
L. quillabamba & 0,233 & 0,215 & 0,251 \\
L. wattsi & 0,237 & 0,211 & 0,263 \\
L. rispaili & 0,238 & 0,217 & 0,259 \\
S. microps & 0,24 & 0,224 & 0,256 \\
L. gonzaloi & 0,243 & 0,225 & 0,261 \\
L. scorzai & 0,245 & 0,219 & 0,271 \\
L. monzonensis & 0,249 & 0,231 & 0,267 \\
L. kirigetiensis & 0,26 & 0,246 & 0,274 \\
L. guderiani & 0,266 & 0,248 & 0,284 \\
H. hertigi & 0,284 & 0,258 & 0,310 \\
S. sordelli & 0,289 & 0,273 & 0,305 \\
\hline & & & \\
& & & \\
& & &
\end{tabular}


Tabela 10 - Média, limites inferior e superior do intervalo de confiança da proporção entre os comprimentos do terceiro palpômero e do palpo de fêmeas (PII/palpo) das espécies analisadas.

\begin{tabular}{|c|c|c|c|}
\hline Espécies & Média & Limite inferior & Limite superior \\
\hline W. lumbrerasi & 0,15 & 0,131 & 0,169 \\
\hline W phlebotomanica & 0,155 & 0,139 & 0,171 \\
\hline B. almeidai & 0,162 & 0,146 & 0,178 \\
\hline$N . \mathrm{sp}$ & 0,162 & 0,147 & 0,177 \\
\hline L. alencari & 0,176 & 0,153 & 0,199 \\
\hline L. lichyi & 0,18 & 0,161 & 0,199 \\
\hline D. samueli & 0,183 & 0,164 & 0,202 \\
\hline L. cruzi & 0,185 & 0,172 & 0,198 \\
\hline L. ischnacantha & 0,185 & 0,169 & 0,201 \\
\hline L. cavernicola & 0,186 & 0,171 & 0,201 \\
\hline L. pallidithorax & 0,186 & 0,171 & 0,201 \\
\hline E. piauiensis & 0,189 & 0,170 & 0,208 \\
\hline L. ischyracantha & 0,189 & 0,170 & 0,208 \\
\hline L. renei & 0,189 & 0,177 & 0,201 \\
\hline L. almerioi & 0,194 & 0,185 & 0,203 \\
\hline L. dispar & 0,195 & 0,185 & 0,205 \\
\hline L. ayacuchensis & 0,195 & 0,185 & 0,205 \\
\hline L. longipalpis & 0,196 & 0,186 & 0,206 \\
\hline L. noguchii & 0,196 & 0,177 & 0,215 \\
\hline L. cruciata & 0,196 & 0,183 & 0,209 \\
\hline L. battistinii & 0,198 & 0,186 & 0,210 \\
\hline L. bicornuta & 0,199 & 0,187 & 0,211 \\
\hline M. vexator & 0,2 & 0,184 & 0,216 \\
\hline L. tejadai & 0,2 & 0,185 & 0,215 \\
\hline L. peruensis & 0,201 & 0,191 & 0,211 \\
\hline L. forattinii & 0,202 & 0,191 & 0,213 \\
\hline L. gomezi & 0,204 & 0,185 & 0,223 \\
\hline L. blancasi & 0,205 & 0,189 & 0,221 \\
\hline O. toroensis & 0,208 & 0,189 & 0,227 \\
\hline L. falcata & 0,208 & 0,185 & 0,231 \\
\hline L. gaminarai & 0,209 & 0,186 & 0,232 \\
\hline L. souzalopesi & 0,212 & 0,196 & 0,228 \\
\hline L. pseudolongipalpis & 0,215 & 0,203 & 0,227 \\
\hline L. sherlocki & 0,217 & 0,194 & 0,240 \\
\hline L. caballeroi & 0,218 & 0,207 & 0,229 \\
\hline L. legerae & 0,22 & 0,201 & 0,239 \\
\hline L. munaypata & 0,225 & 0,209 & 0,241 \\
\hline L. galatiae & 0,225 & 0,212 & 0,238 \\
\hline L. amarali & 0,231 & 0,216 & 0,246 \\
\hline L. quillabamba & 0,235 & 0,219 & 0,251 \\
\hline L. pescei & 0,239 & 0,220 & 0,258 \\
\hline L. castanea & 0,243 & 0,232 & 0,254 \\
\hline L. ceferinoi & 0,243 & 0,227 & 0,259 \\
\hline L. strictivilla & 0,25 & 0,227 & 0,273 \\
\hline S. microps & 0,254 & 0,235 & 0,273 \\
\hline L. gonzaloi & 0,255 & 0,240 & 0,270 \\
\hline L. scorzai & 0,263 & 0,244 & 0,282 \\
\hline L. adamsi & 0,264 & 0,241 & 0,287 \\
\hline L. monzonensis & 0,268 & 0,253 & 0,283 \\
\hline L. sanguinaria & 0,278 & 0,265 & 0,291 \\
\hline$L$. sp de Pichinde & 0,278 & 0,255 & 0,301 \\
\hline L. hartmanni & 0,282 & 0,263 & 0,301 \\
\hline L. guderiani & 0,284 & 0,268 & 0,300 \\
\hline S. sordellii & 0,295 & 0,280 & 0,310 \\
\hline L. tortura & 0,296 & 0,273 & 0,319 \\
\hline L. cirrita & 0,305 & 0,286 & 0,324 \\
\hline
\end{tabular}


Tabela 11 - Média, limites inferior e superior do intervalo de confiança da proporção entre os comprimentos do quarto palpômero e do palpo de machos (PIV/palpo) das espécies analisadas.

\begin{tabular}{|c|c|c|c|}
\hline Espécies & Média & Limite inferior & Limite superior \\
\hline L. guderiani & 0,103 & 0,090 & 0,116 \\
\hline L. monzonensis & 0,116 & 0,103 & 0,129 \\
\hline L. scorzai & 0,116 & 0,098 & 0,134 \\
\hline L. rispaili & 0,121 & 0,106 & 0,136 \\
\hline L. wattsi & 0,122 & 0,104 & 0,140 \\
\hline L. gonzaloi & 0,126 & 0,113 & 0,139 \\
\hline L. caballeroi & 0,129 & 0,114 & 0,144 \\
\hline L. castanea & 0,129 & 0,119 & 0,139 \\
\hline L. kirigetiensis & 0,132 & 0,122 & 0,142 \\
\hline L. bifoliata & 0,133 & 0,115 & 0,151 \\
\hline L. bicormuta & 0,138 & 0,126 & 0,150 \\
\hline L. lichyi & 0,14 & 0,122 & 0,158 \\
\hline L. renei & 0,142 & 0,131 & 0,153 \\
\hline L. munaypata & 0,142 & 0,129 & 0,155 \\
\hline L. gomezi & 0,143 & 0,125 & 0,161 \\
\hline L. cavernicola & 0,144 & 0,132 & 0,156 \\
\hline L. almerioi & 0,146 & 0,138 & 0,154 \\
\hline L. tejadai & 0,146 & 0,138 & 0,154 \\
\hline L. galatiae & 0,147 & 0,136 & 0,158 \\
\hline L. cruciata & 0,149 & 0,138 & 0,160 \\
\hline L. quillabamba & 0,15 & 0,137 & 0,163 \\
\hline L. souzalopesi & 0,151 & 0,141 & 0,161 \\
\hline L. ayacuchensis & 0,151 & 0,143 & 0,159 \\
\hline L. peruensis & 0,151 & 0,144 & 0,158 \\
\hline L. ischnacantha & 0,153 & 0,141 & 0,165 \\
\hline L. noguchii & 0,155 & 0,142 & 0,168 \\
\hline L. blancasi & 0,156 & 0,146 & 0,166 \\
\hline L. pallidithorax & 0,156 & 0,144 & 0,168 \\
\hline L. falcata & 0,156 & 0,138 & 0,174 \\
\hline L. battistinii & 0,157 & 0,147 & 0,167 \\
\hline L. dispar & 0,157 & 0,146 & 0,168 \\
\hline L. castroi & 0,157 & 0,139 & 0,175 \\
\hline L. forattinii & 0,158 & 0,149 & 0,167 \\
\hline L. chavinensis & 0,158 & 0,145 & 0,171 \\
\hline L. alencari & 0,16 & 0,142 & 0,178 \\
\hline L. legerae & 0,161 & 0,146 & 0,176 \\
\hline L. cruzi & 0,166 & 0,155 & 0,177 \\
\hline L. ischyracantha & 0,167 & 0,152 & 0,182 \\
\hline S. microps & 0,168 & 0,156 & 0,180 \\
\hline L. longipalpis & 0,168 & 0,160 & 0,176 \\
\hline L. pseudolongipalpis & 0,173 & 0,160 & 0,186 \\
\hline S. sordelli & 0,177 & 0,165 & 0,189 \\
\hline M. vexator & 0,18 & 0,168 & 0,192 \\
\hline B. almeidai & 0,181 & 0,168 & 0,194 \\
\hline H. hertigi & 0,184 & 0,166 & 0,202 \\
\hline L. gaminarai & 0,19 & 0,172 & 0,208 \\
\hline O. toroensis & 0,192 & 0,177 & 0,207 \\
\hline$N . \mathrm{sp}$ & 0,193 & 0,180 & 0,206 \\
\hline W. phlebotomanica & 0,195 & 0,177 & 0,213 \\
\hline$N$. pilipes & 0,198 & 0,185 & 0,211 \\
\hline E. piauiensis & 0,203 & 0,188 & 0,218 \\
\hline
\end{tabular}


Tabela 12 - Média. limites inferior e superior do intervalo de confiança da proporção entre os comprimentos do quarto palpômero e do palpo de fêmeas (PIV/palpo) das espécies analisadas.

\begin{tabular}{|c|c|c|c|}
\hline Espécies & Média & Limite inferior & Limite superior \\
\hline L. guderiani & 0,103 & 0,091 & 0.115 \\
\hline I. harmmanni & 0,11 & 0,096 & 0.124 \\
\hline L. adamsi & 0.113 & 0.096 & 0,130 \\
\hline 1. castanea & 0,115 & 0,107 & 0.123 \\
\hline L. monzonensis & 0.115 & 0.104 & 0.126 \\
\hline l. gonzaloi & 0.117 & 0.106 & 0,128 \\
\hline L. scorzai & 0.119 & 0.105 & 0.133 \\
\hline 1. sanguinaria & 0.121 & 0.111 & 0.131 \\
\hline L. sp de Pichinde & 0.121 & 0.104 & 0.138 \\
\hline L. strictivilla & 0,122 & 0.105 & 0.139 \\
\hline L. cirrita & 0.122 & 0.108 & 0.136 \\
\hline L. caballeroi & 0,125 & 0.117 & 0.133 \\
\hline L. ceferinoi & 0.128 & 0.116 & 0.140 \\
\hline L. muncypata & 0.129 & 0.117 & 0.141 \\
\hline L. tortura & 0.129 & 0.112 & 0.146 \\
\hline L. carernicola & 0.134 & 0.123 & 0,145 \\
\hline L. bicornuta & 0.135 & 0.126 & 0.144 \\
\hline L. tejadai & 0.135 & 0.124 & 0.146 \\
\hline L. almerioi & 0.141 & 0.134 & 0.148 \\
\hline 1. aracuchensis & 0.142 & 0.134 & 0.150 \\
\hline L. batristinii & 0.143 & 0.134 & 0.152 \\
\hline L. renei & 0,143 & 0.134 & 0,152 \\
\hline L. quillabamba & 0.143 & 0.131 & 0.155 \\
\hline L. peruensis & 0.143 & 0.135 & 0.151 \\
\hline L. blancasi & 0,144 & 0.132 & 0.156 \\
\hline L. ischnacantha & 0.147 & 0,135 & 0.159 \\
\hline L. pallidithorax & 0.147 & 0.136 & 0.158 \\
\hline L. dispar & 0.149 & 0,141 & 0.157 \\
\hline L. forattinii & 0,149 & 0.141 & 0.157 \\
\hline L. lichii & 0.15 & 0.136 & 0.164 \\
\hline L. amarali & 0.15 & 0.139 & 0.161 \\
\hline L. souzalopesi & 0.151 & 0.139 & 0.163 \\
\hline L. percei & 0.151 & 0.141 & 0.161 \\
\hline L. cruciata & 0.151 & 0.141 & 0.161 \\
\hline L. gomezi & 0.151 & 0.137 & 0.165 \\
\hline L. sherlocki & 0,152 & 0.135 & 0.169 \\
\hline L. legerae & 0.155 & 0.141 & 0.169 \\
\hline L. falcata & 0,156 & 0.139 & 0.173 \\
\hline L. galatiae & 0.157 & 0.147 & 0.167 \\
\hline L. noguchii & 0,157 & 0.147 & 0.167 \\
\hline L. alencari & 0.159 & 0,142 & 0,176 \\
\hline L. longipalpis & 0.159 & 0,152 & 0.166 \\
\hline S. sordelliii & 0.163 & 0.152 & 0.174 \\
\hline L. ischvracantha & 0,163 & 0,149 & 0.177 \\
\hline L. cruzi & 0,165 & 0,155 & 0.175 \\
\hline S. microps & 0,171 & 0.157 & 0.185 \\
\hline L. gaminarai & 0,174 & 0.157 & 0,191 \\
\hline 1. vexator & 0,175 & 0,163 & 0,187 \\
\hline B. almeidai & 0,177 & 0,165 & 0.189 \\
\hline V. sp & 0,178 & 0,167 & 0.189 \\
\hline O. toroensis & 0.179 & 0.165 & 0,193 \\
\hline L. pseudolongipalpis & 0,18 & 0,171 & 0.189 \\
\hline W. phlebotomanica & 0.183 & 0,171 & 0,195 \\
\hline D. samueli & 0,187 & 0,173 & 0,201 \\
\hline W. lumbrerasi & 0.189 & 0.175 & 0.203 \\
\hline E. piauiensis & 0,207 & 0,193 & 0.221 \\
\hline
\end{tabular}


Tabela 13 - Média, limites inferior e superior do intervalo de confiança da proporção entre os comprimentos do quinto palpômero e do palpo de machos (PV/palpo) das espécies analisadas.

\begin{tabular}{|c|c|c|c|}
\hline Espécies & Média & Limite inferior & Limite superior \\
\hline H. hertigi & 0,199 & 0,146 & 0,252 \\
\hline S. sordelli & 0,319 & 0,276 & 0,362 \\
\hline L. kirigetiensis & 0,324 & 0,291 & 0,357 \\
\hline L. quillabamba & 0,341 & 0,304 & 0,378 \\
\hline L. gaminarai & 0,346 & 0,309 & 0,383 \\
\hline O. toroensis & 0,356 & 0,330 & 0,382 \\
\hline L. monzonensis & 0,361 & 0,318 & 0,404 \\
\hline L. guderiani & 0,362 & 0,334 & 0,390 \\
\hline W. phlebotomanica & 0,365 & 0,328 & 0,402 \\
\hline L. legerae & 0,373 & 0,320 & 0,426 \\
\hline L. gonzaloi & 0,376 & 0,343 & 0,409 \\
\hline L. wattsi & 0,383 & 0,330 & 0,436 \\
\hline S. microps & 0,384 & 0,341 & 0,427 \\
\hline L. chavinensis & 0,384 & 0,351 & 0,417 \\
\hline L. munaypata & 0,386 & 0,358 & 0,414 \\
\hline L. rispaili & 0,388 & 0,360 & 0,416 \\
\hline L. scorzai & 0,396 & 0,359 & 0,433 \\
\hline L. battistinii & 0,399 & 0,362 & 0,436 \\
\hline L. galatiae & 0,401 & 0,377 & 0,425 \\
\hline L. castanea & 0,402 & 0,365 & 0,439 \\
\hline L. castroi & 0,404 & 0,361 & 0,447 \\
\hline L. bifoliata & 0,405 & 0,372 & 0,438 \\
\hline L. souzalopesi & 0,406 & 0,376 & 0,436 \\
\hline L. ayacuchensis & 0,41 & 0,357 & 0,463 \\
\hline L. dispar & 0,413 & 0,383 & 0,443 \\
\hline L. noguchii & 0,424 & 0,394 & 0,454 \\
\hline L. blancasi & 0,425 & 0,401 & 0,449 \\
\hline L. peruensis & 0,425 & 0,392 & 0,458 \\
\hline L. falcata & 0,427 & 0,374 & 0,480 \\
\hline L. pseudolongipalpis & 0,428 & 0,404 & 0,452 \\
\hline$M$. vexator & 0,429 & 0,396 & 0,462 \\
\hline L. tejadai & 0,429 & 0,405 & 0,453 \\
\hline L. cruciata & 0,431 & 0,401 & 0,461 \\
\hline E. pioniensis & 0,432 & 0,389 & 0,475 \\
\hline L. forattinii & 0,433 & 0,403 & 0,463 \\
\hline L. caballeroi & 0,434 & 0,406 & 0,462 \\
\hline L. longipalpis & 0,437 & 0,384 & 0,490 \\
\hline L. almerioi & 0,444 & 0,391 & 0,497 \\
\hline L. pallidithorax & 0,444 & 0,407 & 0,481 \\
\hline L. cavernicola & 0,448 & 0,395 & 0,501 \\
\hline L. ischnacantha & 0,451 & 0,398 & 0,504 \\
\hline L. gomezi & 0,454 & 0,401 & 0,507 \\
\hline L. cruzi & 0,455 & 0,422 & 0,488 \\
\hline L. lichyi & 0,455 & 0,412 & 0,498 \\
\hline L.bicornuta & 0,459 & 0,431 & 0,487 \\
\hline$N . \mathbf{s p}$ & 0,461 & 0,424 & 0,498 \\
\hline L. ischyracantha & 0,466 & 0,433 & 0,499 \\
\hline L. alencari & 0,471 & 0,438 & 0,504 \\
\hline L. renei & 0,486 & 0,449 & 0,523 \\
\hline B. almeidai & 0,491 & 0,454 & 0,528 \\
\hline$N$. pilipes & 0,504 & 0,467 & 0,541 \\
\hline
\end{tabular}


Tabela 14 - Média. limites inferior e superior do intervalo de confiança da proporção entre os comprimentos do quinto palpômero e do palpo de fêmeas ( $\mathrm{PV} / \mathrm{palpo}$ ) das espécies analisadas.

\begin{tabular}{|c|c|c|c|}
\hline Espécies & Média & Limite inferior & Limite superior \\
\hline L. cirrita & 0.259 & 0,212 & 0,306 \\
\hline L. hartmanni & 0.276 & 0,229 & 0,323 \\
\hline L. tortura & 0.28 & 0.222 & 0.338 \\
\hline L. sanguinaria & 0.3 & 0,267 & 0.333 \\
\hline L. sp de Pichinde & 0.301 & 0.243 & 0,359 \\
\hline L. adamsi & 0,305 & 0,247 & 0.363 \\
\hline L. monzonensis & 0,306 & 0,270 & 0.342 \\
\hline L. guderiani & 0.309 & 0.268 & 0,350 \\
\hline L. quillabamba & 0.311 & 0,270 & 0.352 \\
\hline S. sorclellii & 0.313 & 0.277 & 0.349 \\
\hline L. scorzai & 0.321 & 0.274 & 0.368 \\
\hline L. galatiae & 0.322 & 0.289 & 0.355 \\
\hline L. gonzaloi & 0.325 & 0,289 & 0.361 \\
\hline L. munavpata & 0.338 & 0.297 & 0.379 \\
\hline L. castanea & 0.347 & 0.320 & 0.374 \\
\hline L. ceferinoi & 0.347 & 0.306 & 0.388 \\
\hline L. renei & 0.352 & 0,321 & 0.383 \\
\hline L. pescei & 0.36 & 0.327 & 0.393 \\
\hline S. microps & 0.365 & 0,318 & 0.412 \\
\hline L. strictivilla & 0.365 & 0,307 & 0.423 \\
\hline O. toroensis & 0.368 & 0.321 & 0.415 \\
\hline IV. lumbrerasi & 0,373 & 0,326 & 0,420 \\
\hline L. ischnacantha & 0.373 & 0.332 & 0.414 \\
\hline L. amarali & 0,378 & 0,342 & 0.414 \\
\hline W. phlebotomanica & 0,385 & 0,344 & 0.426 \\
\hline L. legerae & 0,386 & 0,339 & 0.433 \\
\hline L. caballeroi & 0.399 & 0.372 & 0.426 \\
\hline L. gaminarai & 0,407 & 0,349 & 0,465 \\
\hline L. avacuchensis & 0.407 & 0.381 & 0.433 \\
\hline L. blancasi & 0,41 & 0.369 & 0.451 \\
\hline L. noguchii & 0.41 & 0,377 & 0,443 \\
\hline L. forattinii & 0,418 & 0,391 & 0.445 \\
\hline L. falcata & 0.42 & 0.362 & 0.478 \\
\hline L. battistinit & 0,4226 & 0,394 & 0.451 \\
\hline L. peruensis & 0,423 & 0.397 & 0,449 \\
\hline E. piauiensis & 0.425 & 0,378 & 0.472 \\
\hline D. samueli & 0.425 & 0.378 & 0,472 \\
\hline M. vexator & 0,425 & 0.384 & 0.466 \\
\hline L. almerioi & 0,425 & 0,401 & 0.449 \\
\hline L. tejadai & 0.425 & 0,389 & 0.461 \\
\hline L. cavernicola & 0,427 & 0,391 & 0.463 \\
\hline L. dispar & 0.427 & 0,401 & 0.453 \\
\hline L. cruciata & 0.427 & 0,394 & 0,460 \\
\hline L. gomezi & 0,427 & 0,380 & 0,474 \\
\hline L. sherlocki & 0,427 & 0,369 & 0,485 \\
\hline L. pseudolongipalpis & 0,432 & 0,401 & 0.463 \\
\hline L. souzalopesi & 0,432 & 0,391 & 0,473 \\
\hline L. bicornuta & 0,435 & 0,406 & 0,464 \\
\hline L. lichvi & 0,443 & 0,396 & 0.490 \\
\hline L. pallidithorax & 0,444 & 0,408 & 0,480 \\
\hline L. ischyracantha & 0.446 & 0,399 & 0,493 \\
\hline L. cruzi & 0,451 & 0,418 & 0,484 \\
\hline L. longipalpis & 0,461 & 0,436 & 0,486 \\
\hline L. alencari & 0,407 & 0.465 & 0.523 \\
\hline N. sp & 0,492 & 0,456 & 0,528 \\
\hline B. almeidai & 0,501 & 0,460 & 0.542 \\
\hline
\end{tabular}


Tabela 15 - Média, limites inferior e superior do intervalo de confiança da razào entre os comprimentos do segundo e quarto palpômeros de machos (PII/PIV) das espécies analisadas.

\begin{tabular}{|c|c|c|c|}
\hline Espécies & Média & Limite inferior & Limite superior \\
\hline N. pilipes & 0.51 & 0.298 & 0.722 \\
\hline B. almeidai & 0.569 & 0.385 & 0.753 \\
\hline V. $\mathbf{s p}$ & 0.623 & 0.439 & 0.807 \\
\hline E. piauiensis & 0.635 & 0.375 & 0.895 \\
\hline D. samuelli & 0.722 & 0.510 & 0.934 \\
\hline M. vexator & 0.779 & 0.615 & 0.943 \\
\hline S. sordelli & 0.787 & 0,623 & 0.951 \\
\hline B. nitzulescui & 0.801 & 0.541 & 1.061 \\
\hline S. microps & 0.822 & 0,658 & 0,986 \\
\hline L. ischyracantha & 0.842 & 0.630 & 1.054 \\
\hline L. alencari & 0.896 & 0,684 & 1.108 \\
\hline L. cruzi & 0.896 & 0.757 & 1.035 \\
\hline L. pseudolongipalpis & 0.91 & 0,726 & 1.094 \\
\hline L. longipalpis & 0.922 & 0.758 & 1.086 \\
\hline L. gaminarai & 0.962 & 0,702 & 1.222 \\
\hline L. falcata & 1.012 & 0,752 & 1.272 \\
\hline L. pallidithorax & 1.031 & 0,867 & 1.195 \\
\hline L. forattinii & 1.036 & 0.906 & 1.166 \\
\hline L. dispar & 1.052 & 0.902 & 1.202 \\
\hline L. battistinii & 1.066 & 0.902 & 1.230 \\
\hline L. renei & 1.075 & 0.945 & 1.205 \\
\hline L. ischnacantha & 1,078 & 0.914 & 1.242 \\
\hline W phlebotomanica & 1.102 & 0,842 & 1,362 \\
\hline L. bicornuta & 1.102 & 0.938 & 1.266 \\
\hline L. blancasi & 1.145 & 1.006 & 1.284 \\
\hline L. gomezi & 1.149 & 0.889 & $1 .+09$ \\
\hline L. almerioi & 1.171 & 1,055 & 1.287 \\
\hline L. peruensis & 1.181 & 1,051 & 1.311 \\
\hline L. cavernicola & 1,184 & 1,020 & 1.348 \\
\hline L. souzalopesi & 1.185 & 1.046 & 1.324 \\
\hline L. lichyi & 1.199 & 0.939 & $1 .+59$ \\
\hline L. bifoliata & 1.237 & 0.977 & 1.497 \\
\hline L. legerae & 1.258 & 1,046 & $1 .+70$ \\
\hline L. avacuchensis & 1.27 & 1.154 & 1.386 \\
\hline L. chavinensis & 1,282 & 1,098 & 1.466 \\
\hline H. hertigi & 1.297 & 1,037 & 1.557 \\
\hline L. tejadai & 1,298 & 1,182 & 1,414 \\
\hline L. caballeroi & 1.306 & 1,094 & 1.518 \\
\hline L. galatiae & 1,344 & 1.194 & 1.494 \\
\hline L. munavpata & 1.383 & 1.199 & 1.567 \\
\hline L. castanea & 1.45 & 1.311 & 1.589 \\
\hline L. quillabamba & 1,501 & 1.317 & 1,685 \\
\hline L. scorzai & 1,511 & 1.251 & 1,771 \\
\hline L. gonzaloi & 1.565 & 1.381 & 1.749 \\
\hline L. kirigetiensis & 1,599 & 1,460 & 1,738 \\
\hline L. wattsi & 1.639 & 1.379 & 1,899 \\
\hline L. rispaili & 1,691 & 1,479 & 1,903 \\
\hline L. monzonensis & 1,847 & 1.663 & 2,031 \\
\hline L. guderiani & 2,093 & 1,909 & 2,277 \\
\hline
\end{tabular}


Tabela 16 - Média, limites inferior e superior do intervalo de confiança da razão entre os comprimentos do segundo e quarto palpômeros de fêmeas (PII/PIV) das espécies analisadas.

\begin{tabular}{|c|c|c|c|}
\hline Espécies & Média & Limite inferior & Limite superior \\
\hline B. almeidai & 0,607 & 0,406 & 0,808 \\
\hline E. piauiensis & 0,63 & 0,346 & 0,914 \\
\hline$N . \mathbf{s p}$ & 0,695 & 0,494 & 0,896 \\
\hline M. vexator & 0,842 & 0,641 & 1,043 \\
\hline S. microps & 0,863 & 0,631 & 1,095 \\
\hline D. samueli & 0,917 & 0,685 & 1,149 \\
\hline S. sordellii & 0,93 & 0,751 & 1,109 \\
\hline L. alencari & 0,935 & 0,651 & 1,219 \\
\hline L. cruzi & 0,988 & 0,824 & 1,152 \\
\hline L. longipalpis & 0,992 & 0,828 & 1,156 \\
\hline L. ischyracantha & 1,019 & 0,735 & 1,303 \\
\hline L. sherlocki & 1,026 & 0,742 & 1,310 \\
\hline L. pseudolongipalpis & 1,032 & 0,880 & 1,184 \\
\hline L. amarali & 1,039 & 0,755 & 1,323 \\
\hline L. cruciata & 1,052 & 0,768 & 1,336 \\
\hline L. evangelistai & 1,056 & 0,772 & 1,340 \\
\hline L. gaminarai & 1,069 & 0,785 & 1,353 \\
\hline L. gomezi & 1,096 & 0,864 & 1,328 \\
\hline L. lichyi & 1,105 & 0,821 & 1,389 \\
\hline B. cardosoi & 1,124 & 0,840 & 1,408 \\
\hline L. battistinii & 1,135 & 0,956 & 1,314 \\
\hline L. souzalopesi & 1,144 & 0,965 & 1,323 \\
\hline L. dispar & 1,163 & 0,999 & 1,327 \\
\hline L. ischnacantha & 1,176 & 0,975 & 1,377 \\
\hline L. tejadai & 1.209 & 1.030 & 1.388 \\
\hline L. marinkellei & 1,227 & 0,943 & 1,511 \\
\hline L. galatiae & 1,23 & 1,066 & 1,394 \\
\hline W. phlebotomanica & 1,237 & 1.005 & 1.469 \\
\hline L. forattinii & 1,25 & 1,116 & 1,384 \\
\hline L. renei & 1,266 & 1,124 & 1,408 \\
\hline W. lumbrerasi & 1,27 & 1,038 & 1,502 \\
\hline L. pallidithorax & 1,29 & 1.111 & 1,469 \\
\hline L. legerae & 1,32 & 1,088 & 1,552 \\
\hline L. cavernicola & 1,329 & 1,165 & 1,493 \\
\hline L. blancasi & 1,337 & 1,173 & 1,501 \\
\hline L. almerioi & 1.355 & 1,234 & 1,476 \\
\hline L. ayacuchensis & 1,368 & 1,189 & 1,547 \\
\hline L. bicornuta & 1,38 & 1,179 & 1,581 \\
\hline L. bifoliata & 1,416 & 1,132 & 1,700 \\
\hline L. peruensis & 1,434 & 1,202 & 1,666 \\
\hline L. pescei & 1,549 & 1,370 & 1,728 \\
\hline L. sp de Pichinde & 1,561 & 1,434 & 1,688 \\
\hline L. strictivilla & 1.584 & 1,300 & 1,868 \\
\hline L. caballeroi & 1,617 & 1,385 & 1,849 \\
\hline L. quillabamba & 1,764 & 1,564 & 1,964 \\
\hline L. ceferinoi & 1,787 & 1,586 & 1,988 \\
\hline L. tortura & 1,791 & 1,507 & 2,075 \\
\hline L. munaypata & 1,918 & 1,717 & 2,119 \\
\hline L. sanguinaria & 1,926 & 1,762 & 2,090 \\
\hline L. castanea & 2,008 & 1,844 & 2,172 \\
\hline L. gonzaloi & 2,035 & 1,856 & 2,214 \\
\hline L. scorzai & 2,077 & 1,845 & 2,309 \\
\hline L. hartmanni & 2,145 & 1,944 & 2,346 \\
\hline L. adamsi & 2,178 & 1,894 & 2,462 \\
\hline L. monzonensis & 2,182 & 2,003 & 2,361 \\
\hline L. guderiani & 2,435 & 2,234 & 2,636 \\
\hline
\end{tabular}


Tabela 17 - Média, limites inferior e superior do intervalo de confiança da razto entre os comprimentos do quinto terceiro palpômeros de machos (PV/PIII) das espécies analisadas.

\begin{tabular}{|c|c|c|c|}
\hline Espécies & Média & Limite inferior & Limite superior \\
\hline H. hertigi & 0,702 & 0,241 & 1,163 \\
\hline S. sordellii & 1,109 & 0,817 & 1,401 \\
\hline L. ceferinoi & 1,115 & 0,654 & 1,576 \\
\hline L. gaminarai & 1,166 & 0,705 & 1,627 \\
\hline L. kirigetiensis & 1,25 & 1,004 & 1,496 \\
\hline L. guderiani & 1,361 & 1,035 & 1,687 \\
\hline L. monzonensis & 1,449 & 1,123 & 1,775 \\
\hline L. quillabamba & 1,467 & 1,141 & 1,793 \\
\hline L. gonzaloi & 1,549 & 1,223 & 1,875 \\
\hline S. microps & 1,605 & 1,313 & 1,897 \\
\hline L. scorzai & 1,618 & 1,157 & 2,079 \\
\hline L. wattsi & 1,624 & 1,163 & 2,085 \\
\hline L. rispaili & 1,631 & 1,255 & 2,007 \\
\hline L. legerae & 1,723 & 1,347 & 2,099 \\
\hline L. bifoliata & 1,747 & 1,286 & 2,208 \\
\hline L. castanea & 1,76 & 1,514 & 2,006 \\
\hline L. munaypata & 1,786 & 1,460 & 2,112 \\
\hline L. chavinensis & 1,834 & 1,508 & 2,160 \\
\hline L. soucalopesi & 1,895 & 1,649 & 2,141 \\
\hline L. galatiae & 1,949 & 1,683 & 2,215 \\
\hline L. caballeroi & 1,974 & 1,598 & 2,350 \\
\hline L. blancasi & 1,979 & 1,733 & 2,225 \\
\hline W. phlebotomanica & 2,091 & 1,630 & 2,552 \\
\hline L. ayacuchensis & 2,111 & 1,905 & 2,317 \\
\hline L. dispar & 2,117 & 1,851 & 2,383 \\
\hline L. tejadai & 2,117 & 1,911 & 2,323 \\
\hline$M$. vexator & 2,13 & 1,838 & 2,422 \\
\hline L. longipalpis & 2,133 & 1,887 & 2,379 \\
\hline L. falcata & 2,139 & 1,678 & 2,600 \\
\hline L. battistinii & 2,172 & 1,880 & 2,464 \\
\hline L. peruensis & 2,198 & 1,967 & 2,429 \\
\hline L. forattinii & 2,201 & 1,970 & 2,432 \\
\hline L. pseudolongipalpis & 2,226 & 1,900 & 2,552 \\
\hline L. cruzi & 2,302 & 2,056 & 2,548 \\
\hline L. pallidithorax & 2,304 & 2,012 & 2,596 \\
\hline L. almerioi & 2,305 & 2,099 & 2,511 \\
\hline L. lichyi & 2,324 & 1,863 & 2,785 \\
\hline E. piouiensis & 2,345 & 1,884 & 2,806 \\
\hline L. bicormuta & 2,349 & 2,057 & 2,641 \\
\hline L. cavernicola & 2,357 & 2,065 & 2,649 \\
\hline L. araracuarensis & 2,376 & 2,000 & 2,752 \\
\hline L. gomezi & 2,378 & 1,917 & 2,839 \\
\hline L. ischnacantha & 2,425 & 2,133 & 2,717 \\
\hline L. alencari & 2,53 & 2,154 & 2,906 \\
\hline$N . \mathrm{sp}$ & 2,531 & 2,205 & 2,857 \\
\hline L. ischyracantha & 2,563 & 2,187 & 2,939 \\
\hline L. renei & 2,565 & 2,334 & 2,796 \\
\hline D. samuelli & 2,584 & 2,208 & 2,960 \\
\hline B. almeidai & 2,853 & 2,527 & 3,179 \\
\hline B. nitzulescui & 2,941 & 2,480 & 3,402 \\
\hline N. pilipes & 3,288 & 2,912 & 3,664 \\
\hline
\end{tabular}


Tabela 18 - Média, limites inferior e superior do intervalo de confiança da razào entre os comprimentos do quinto e terceiro palpômeros de fêmeas (PV/PIII) das espécies analisadas.

\begin{tabular}{|c|c|c|c|}
\hline Espécies & Média & Limite inferior & Limite superior \\
\hline L. scorzai & 0,944 & 0,574 & 1.314 \\
\hline L. guderiani & 1.036 & 0,749 & 1,323 \\
\hline S. microps & 1.062 & 0,608 & 1.516 \\
\hline L. tortura & 1,08 & 0,710 & 1.450 \\
\hline L. monzonensis & 1.082 & 0.761 & 1.403 \\
\hline L. gonzaloi & 1,09 & 0,720 & 1,460 \\
\hline L. hartmanni & 1,142 & 0,855 & 1.429 \\
\hline L. strictivilla & 1,16 & 0,839 & 1,481 \\
\hline L. sanguinaria & 1.224 & 0,854 & 1,594 \\
\hline L. adamsi & 1.277 & 0,823 & 1.731 \\
\hline L. pescei & 1,312 & 1,119 & 1.505 \\
\hline L. munavpata & 1,324 & 1,037 & 1.611 \\
\hline L. castanea & 1,427 & 1,106 & 1.748 \\
\hline L. caballeroi & 1,432 & 0,978 & 1.886 \\
\hline M. vexator & 1.438 & 1,176 & 1.700 \\
\hline L. galatiae & 1,452 & 1,190 & 1.714 \\
\hline L. quillabamba & 1.461 & 1.199 & 1.723 \\
\hline L. ceferinoi & 1,502 & 1,288 & 1,716 \\
\hline S. sordellii & 1.517 & 1,063 & 1.971 \\
\hline L. longipalpis & 1.667 & 1,346 & 1.988 \\
\hline L. amarali & 1.775 & 1.405 & 2.145 \\
\hline L. legerae & 1.776 & 1,322 & 2.230 \\
\hline L. forattinii & 1.781 & 1.519 & 2.043 \\
\hline L. bicornuta & 1,783 & 1,521 & 2.045 \\
\hline L. souzalopesi & 1,836 & 1,574 & 2.098 \\
\hline L. rejadai & 1.894 & 1,573 & 2.215 \\
\hline L. renei & 1,948 & 1.578 & 2.318 \\
\hline L. sherlocki & 1,974 & 1.712 & 2.236 \\
\hline L. blancas & 2.004 & 1,683 & 2.325 \\
\hline L. dispar & 2.033 & 1,712 & 2.354 \\
\hline L. battistinii & 2.05 & 1.729 & 2.371 \\
\hline L. falcata & 2,054 & 1,600 & 2.508 \\
\hline L. avacuchensis & 2,1 & 1,646 & 2.554 \\
\hline L. gomezi & 2,112 & 1,825 & 2.399 \\
\hline D. samueli & 2,132 & 1.811 & 2.453 \\
\hline L. almeriol & 2,142 & 1,821 & 2,463 \\
\hline L. lichyi & 2.148 & 1.861 & 2.435 \\
\hline L. cruciata & 2,148 & 1,886 & $2 .+10$ \\
\hline L. cruzi & 2.17 & 1,800 & 2.540 \\
\hline E. piauiensis & 2,214 & 1,760 & 2.668 \\
\hline L. peruensis & 2.25 & 1,796 & 2.704 \\
\hline L. alencari & 2,283 & 2,080 & 2.486 \\
\hline L. pseudolongipalpis & 2.292 & 1.971 & 2.613 \\
\hline L. ischyracantha & 2.318 & 2,056 & 2.580 \\
\hline B. cardosoi & 2.327 & 2.040 & 2.614 \\
\hline L. cavernicola & 2,332 & 2,045 & 2.619 \\
\hline L. ischnacantha & 2,347 & 1,977 & 2,717 \\
\hline L. pallidithorax & 2,39 & 2,103 & 2.677 \\
\hline L. bifoliata & 2,422 & 2,135 & 2,709 \\
\hline L. gaminarai & 2.424 & 1,970 & 2.878 \\
\hline W. lumbrerasi & 2.481 & 2,027 & 2.935 \\
\hline$N . \mathbf{s p}$ & 3,051 & 2,597 & 3,505 \\
\hline B. almeidai & 3.098 & 2,728 & 3.468 \\
\hline
\end{tabular}




\begin{tabular}{|c|c|}
\hline ?áxons & $\begin{array}{l}\text { Caracteres } \\
00000000011111111112222222222333333333444444444455555555556666 \\
123456789012345678901234567890123456789012345678901234567890123\end{array}$ \\
\hline v. sp & $000012000 \times \times \times 0 \times \times \times \times \times \times \times \times \times \times 0001110 \times x$ \\
\hline$\therefore$ pilipes & $0 ? 0 ? 0 ? 0 ? 0 ? 0 ? 0 ? 0 ? 0 ? 00 \times \times \times \times \times ? 00 \times \times 0000002001 \times \times \times 0 \times \times \times \times \times \times \times \times \times \times 0111110 \times x$ \\
\hline i. hertigi & $1 ? 1 ? 1 ? 2 ? 2 ? 0 ? 2 ? 2 ? 3 ? 000010 ? 00 ? ? \times 000000201 \times \times \times \times 00 \times 0 \times 00 \times \times \times 01111110 \times x$ \\
\hline 3. almeidal & $21110000100000000000 \times \times \times \times \times ? 00 \times \times 0000000000 \times \times \times 0 \times \times \times \times \times \times \times \times \times \times 111111 ? ? ?$ \\
\hline D. nigrosaccula & $122212210001101100000010 ? ? 00 \times \times 0000 ? 0001 \times \times \times \times 0 ? 02 \times 00 \times \times \times 0111111 ? ? ?$ \\
\hline v. fourgassiensis & $121222222111222222000010 ? ? 00 \times \times 0000 ? 0001 \times \times \times \times 0200200 \times \times \times 0111111 ? ? ?$ \\
\hline v. rotundipennis & $1112222231012233320100100000 \times \times 0000000001 \times \times \times 0200000 \times \times \times 0111111100$ \\
\hline v. leponti & $1 ? 1 ? 1 ? 2 ? 1 ? 1 ? 2 ? 3 ? 2 ? 01001111 ? ? ? ? 0000000000 \times \times \times 0201200 \times \times \times 0111111 ? ? ?$ \\
\hline v. lumbrerasi & $2213 ? 3 ? 2101011 ? 1 ? 10000101000 \times 00000000001 \times \times \times 0201200 \times \times \times 0111111 ? ? ?$ \\
\hline v. phlebotomanica & $2233222210001111210000101000 \times \times 0000000001 \times \times \times 0201000 \times \times \times 0111111 ? ? ?$ \\
\hline$\therefore$ piauiensis & $3133110011001100110011001100 \times \times 010001001 \times \times \times \times 0010000 \times \times \times 0100000100$ \\
\hline 2. oligodonta & $11122211110011112100010111010 \times 0100002000 \times 000023000 \times \times \times 0110000100$ \\
\hline 2. toroensis & $21122211110011001101000111010 \times 0100000000 \times 000023100 \times \times \times 0110000100$ \\
\hline 3. cardosol & $21111211110111012101010000110 \times 0100120000 \times 000023100 \times \times \times 0000000100$ \\
\hline 3. nitzulescui & $11101211110011011100000000110 \times 0100120000 \times 000003100 \times \times \times 0000000100$ \\
\hline . samueli & $11121201110001011101110111110 \times 010010201 \times \times \times \times 0022100 \times \times \times 0100000100$ \\
\hline ;. microps & $21231101220011012302110100120011001100012000200000 \times \times \times 1111001100$ \\
\hline$\therefore$ sordeliti & $212311013201220132021100001200110011001 \times \times \times \times 0200000 \times \times \times 0011100100$ \\
\hline 1. vexator & $32231101110011012200110111120001011010010000021000 \times \times \times 111110011 \times$ \\
\hline$\therefore$ ischnacantha & $3212121111010211110111001112000101100001000001 \times x \times 1100011100111 \times$ \\
\hline$\therefore$ bifoliata & $22122311210111122202110010120001011000010220101111102011100011 \times$ \\
\hline$\therefore$ souzalopesi & $22111211211111112211110011121001011000010210101211101111100011 x$ \\
\hline i. gaminarai & 211122111100121131111100111210010111000100001022111021110111100 \\
\hline$\therefore$ alencarl & 221222011101000111111100111210010111100100001212111121110111100 \\
\hline$\therefore$ cruzi & 221212011101000111111100111210010110000100101111111111100111100 \\
\hline$\therefore$ ischyracantha & 221222011101010111111100111210010112100102101222111121110111100 \\
\hline$\therefore$ longipalpis & 221112111101100122111100111210010111100100001212111001100111100 \\
\hline$\therefore$ pseudolongipalpis & 221112111101110111111100111210010112000100001122011001110111100 \\
\hline$\therefore$ dispar & $321213111101111121121100111200010110100102102112112121111111111 \times$ \\
\hline .. Iichyi & $21122111111101111112110011120001011200010220111211222111000011 x$ \\
\hline . castroi & 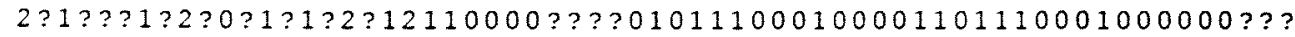 \\
\hline$\because$ amarali & $1113221122011221221211000011000101120001100 \times 110111100100000011 \times$ \\
\hline . c caligata & $1 ? 1 ? 2 ? 1 ? 2 ? 0 ? 2 ? 1 ? 2 ? 12110000 ? ? ? ? ? 10111000110001201110001000000 ? ?$ ?? \\
\hline . falcata & $21111211111121112112110111120001011101013100220111000010000011 \times$ \\
\hline .. cruciata & $21112211111111112112110110120001011201013110210110 \times \times \times 011000011 \times$ \\
\hline . gomezi & $21112211111101111112110111120001011201013100210110 \times \times \times 011000011 \times$ \\
\hline . diabolica & $21112211111121112212110111120001011101011100210200 \times \times \times 011000011 \times$ \\
\hline . marinkellei & 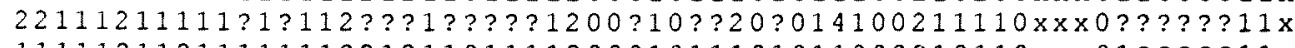 \\
\hline . evangelistai & $11111211211111112212110111120001011101011000210110 \times \times \times 01$ ??????11x \\
\hline - carvalhoi & $21111112211101112212110111120001011201011210212200 \times \times \times 011000011 \times$ \\
\hline . spathotrichia & $21111211111101111112110111120001011201012210220210 \times \times \times 011000011 \times$ \\
\hline - araracuarensis & 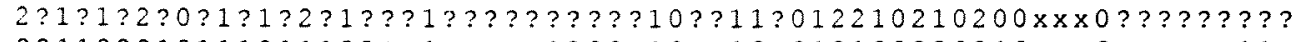 \\
\hline - legerae & $221122212111211122 ? ? 1 ? ? ? ? ? 1200 ? 10 ? ? 10 ? 012100220210 \times \times \times 0 ?$ ?????11x \\
\hline - flabellata & 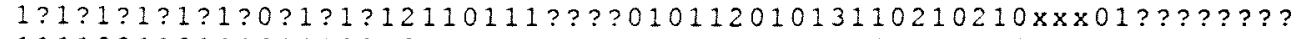 \\
\hline - sherlocki & $11112211211101112212110111120001011201013100210210 \times \times \times 011000011 \times$ \\
\hline - bicornuta & $22232211110101121201110011120001011010010221011201102011110111 \times$ \\
\hline - battistinii & $32232211210111112101110011120001011020010221022201311011110111 x$ \\
\hline - foratinii & 11101111122021100111201010110000102210222111120110001100 \\
\hline - almerioi & 321223111111111211021100111201010110000102210222111120110001100 \\
\hline - cavernicola & $32232311111101111102110011120101011000010210022211110011111111 x$ \\
\hline . renei & 311323111111011112021100111201010110000100110222011101111111100 \\
\hline blancasi & $32332211210111112102110011120001111110013210022210 \times \times \times 0111111100$ \\
\hline - chavinensis & $32332221210110212202110011120001111100014210022210 \times \times \times 0111111100$ \\
\hline - noguchil & $32332211210111122202110011120001111100014210012210 \times \times \times 0111111100$ \\
\hline - paliidithorax & $32332221110101111102110011120001101000014110022000 \times \times \times 0111111100$ \\
\hline ayacuchensis & $32333321111111222102110011120001101100013110020210 \times \times \times 11100001 \times 1$ \\
\hline peruensis & $32333321111111122102110011120001101100013110000200 \times \times \times 011000011 \times$ \\
\hline
\end{tabular}

:genda: 0 = plesiomorfia: $1,2,3,4$, = apomorfia; ? = caráter não observado; $\mathrm{x}=$ não comparável 
Tabela 19. Matriz de dados para a analise cladistica das espécies de Lutzomyia - Continuação

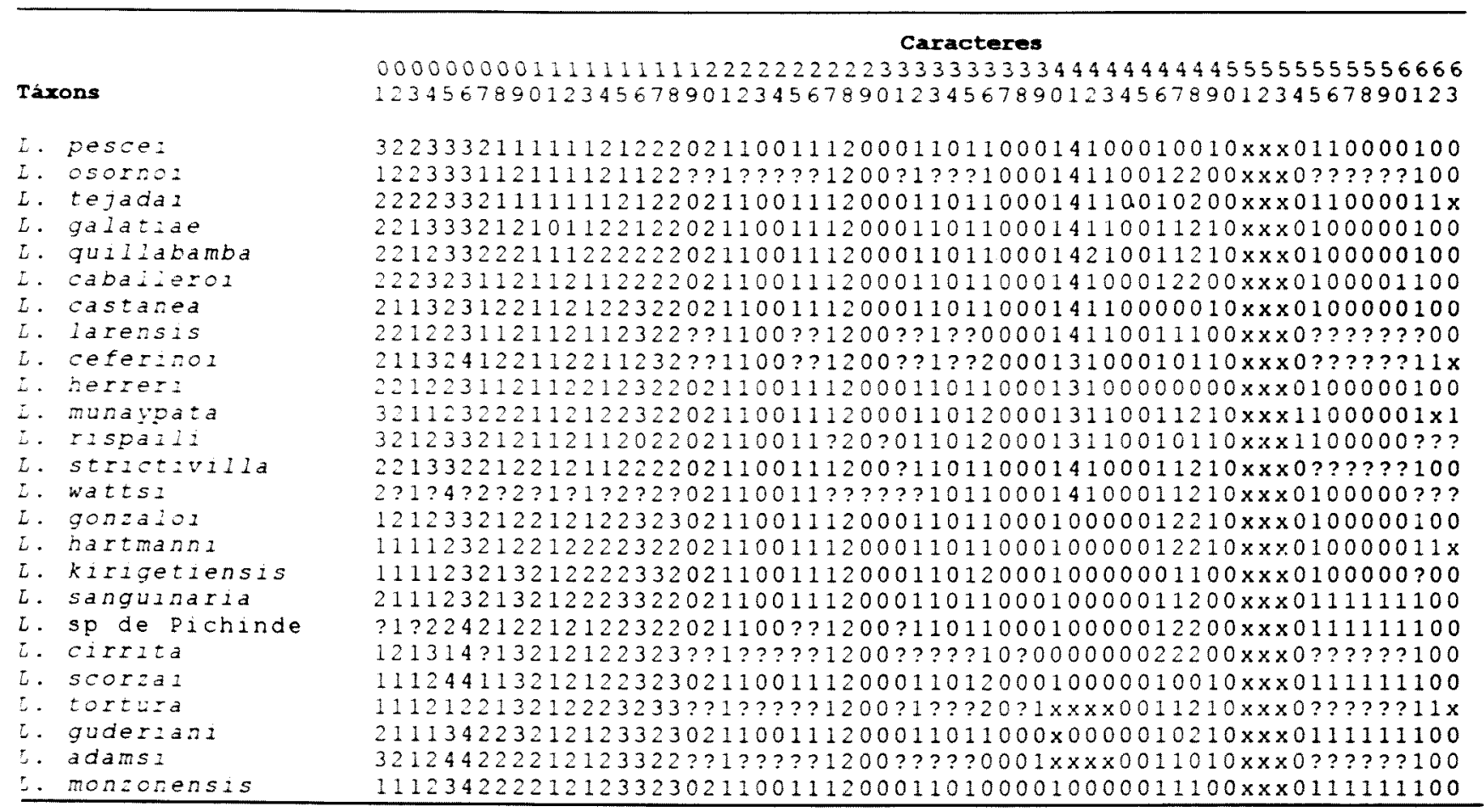

egenda: 0 = plesiomorfia: 1.2 .3 .4 = apomorfia: ? = carater não observado: $\mathrm{x}=$ não comparável 
FIGURAS 


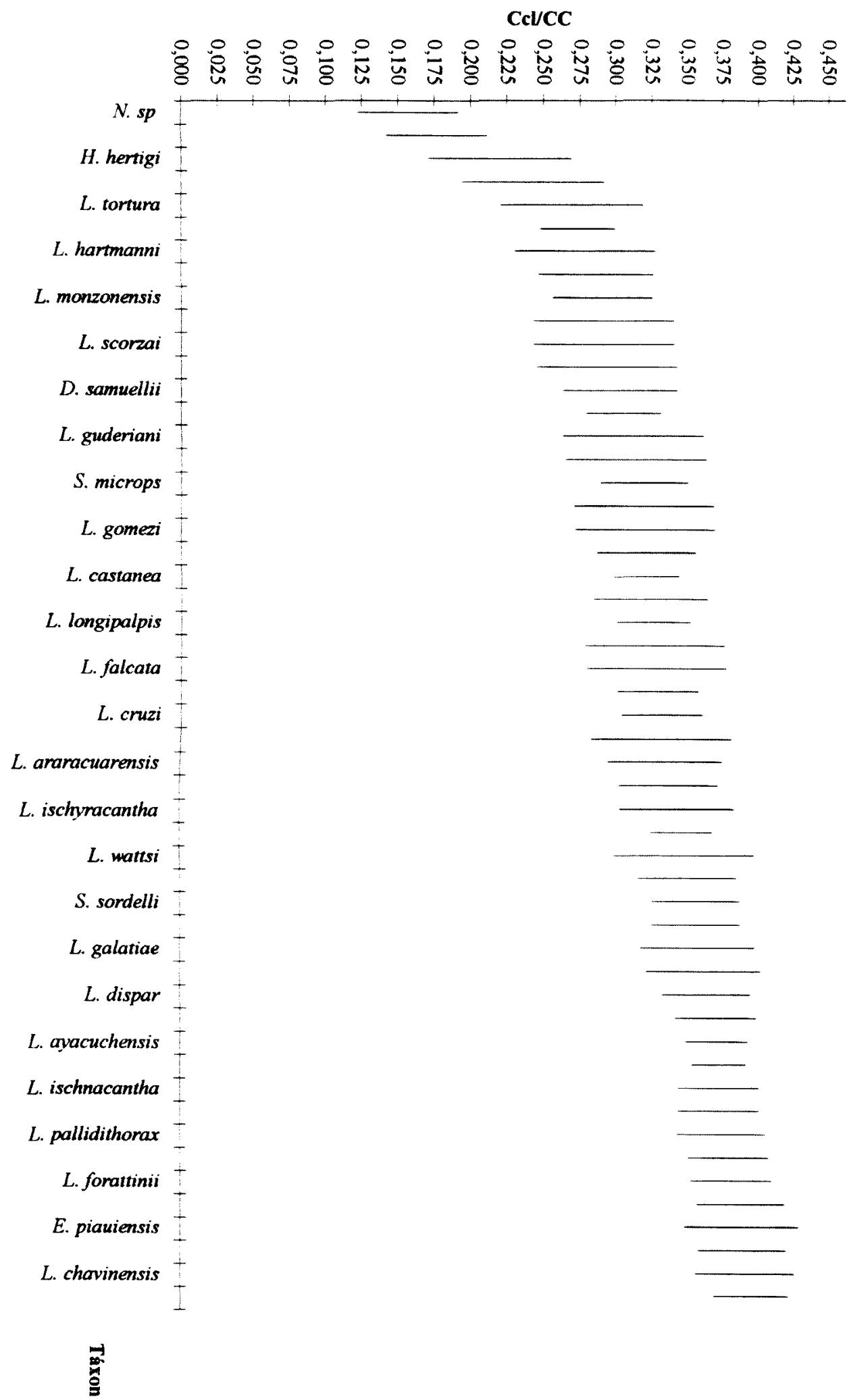




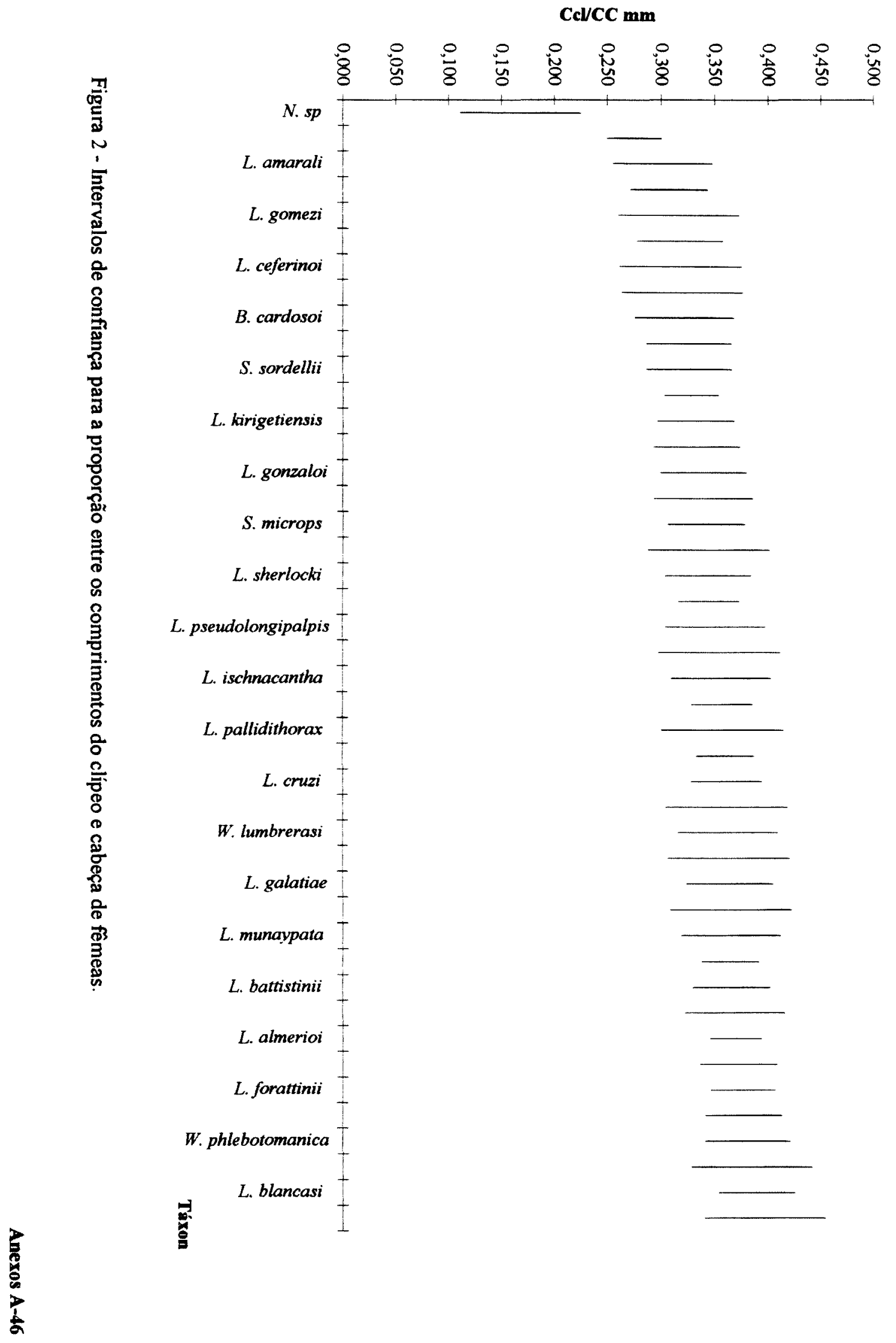




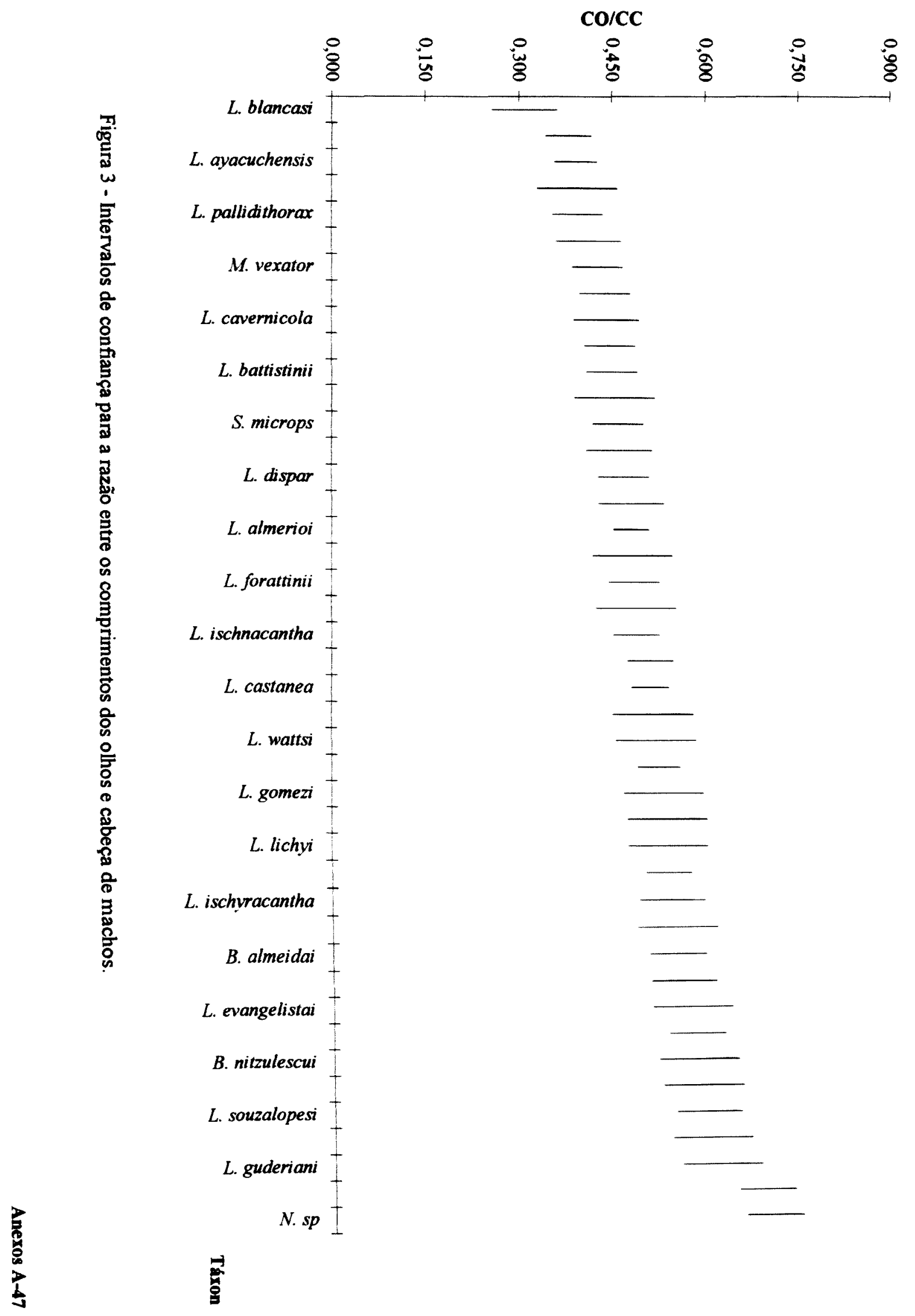




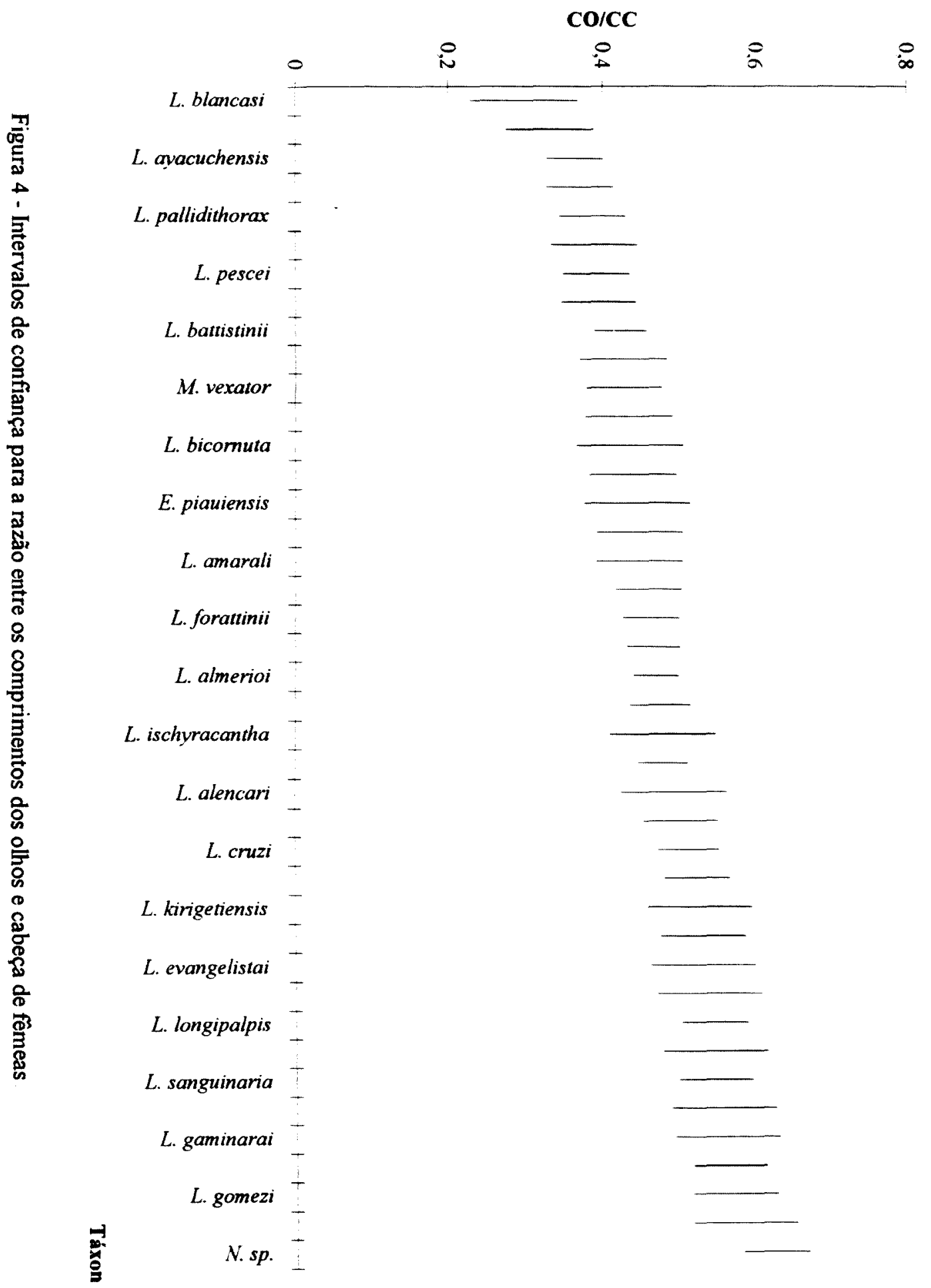




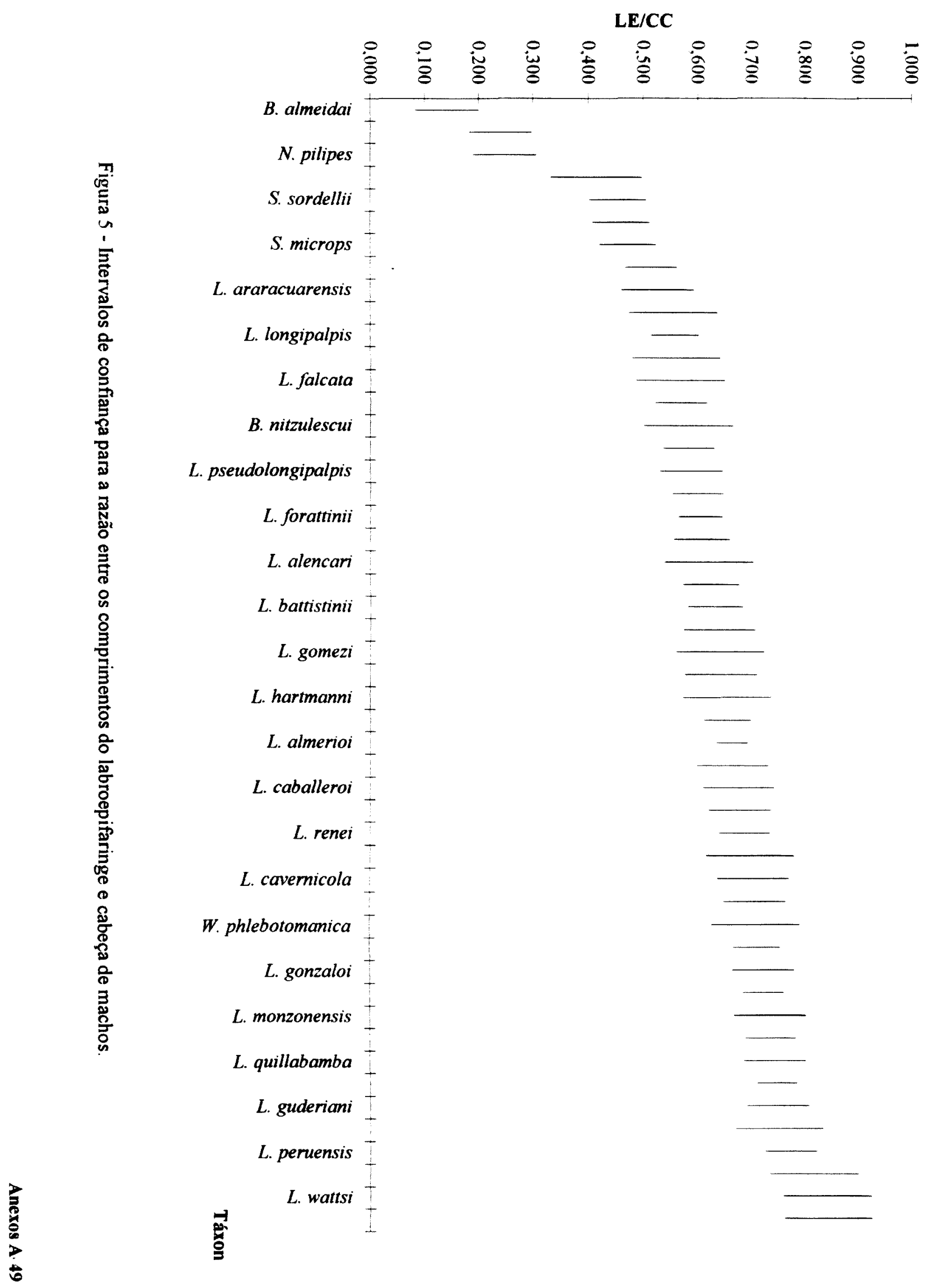




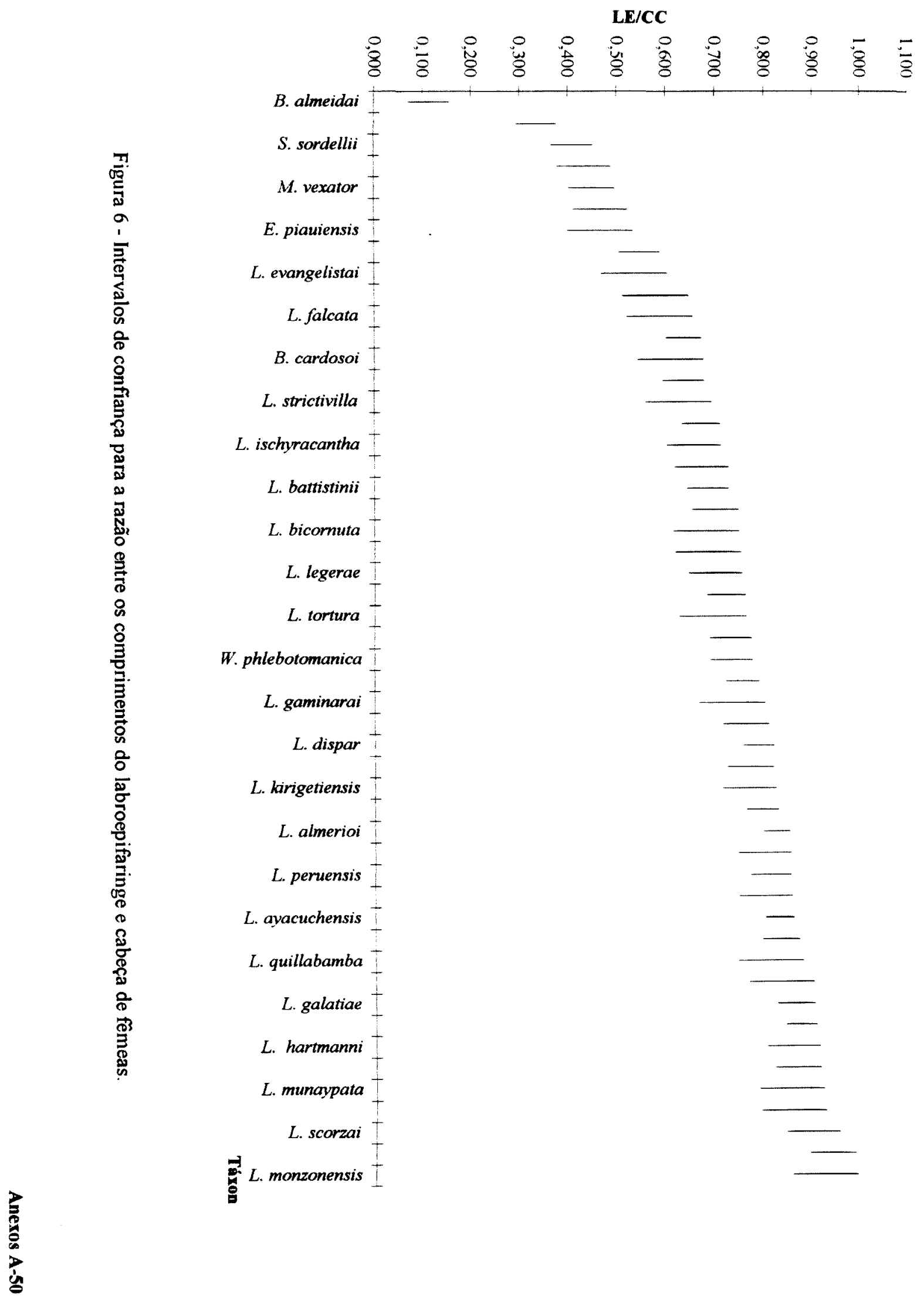




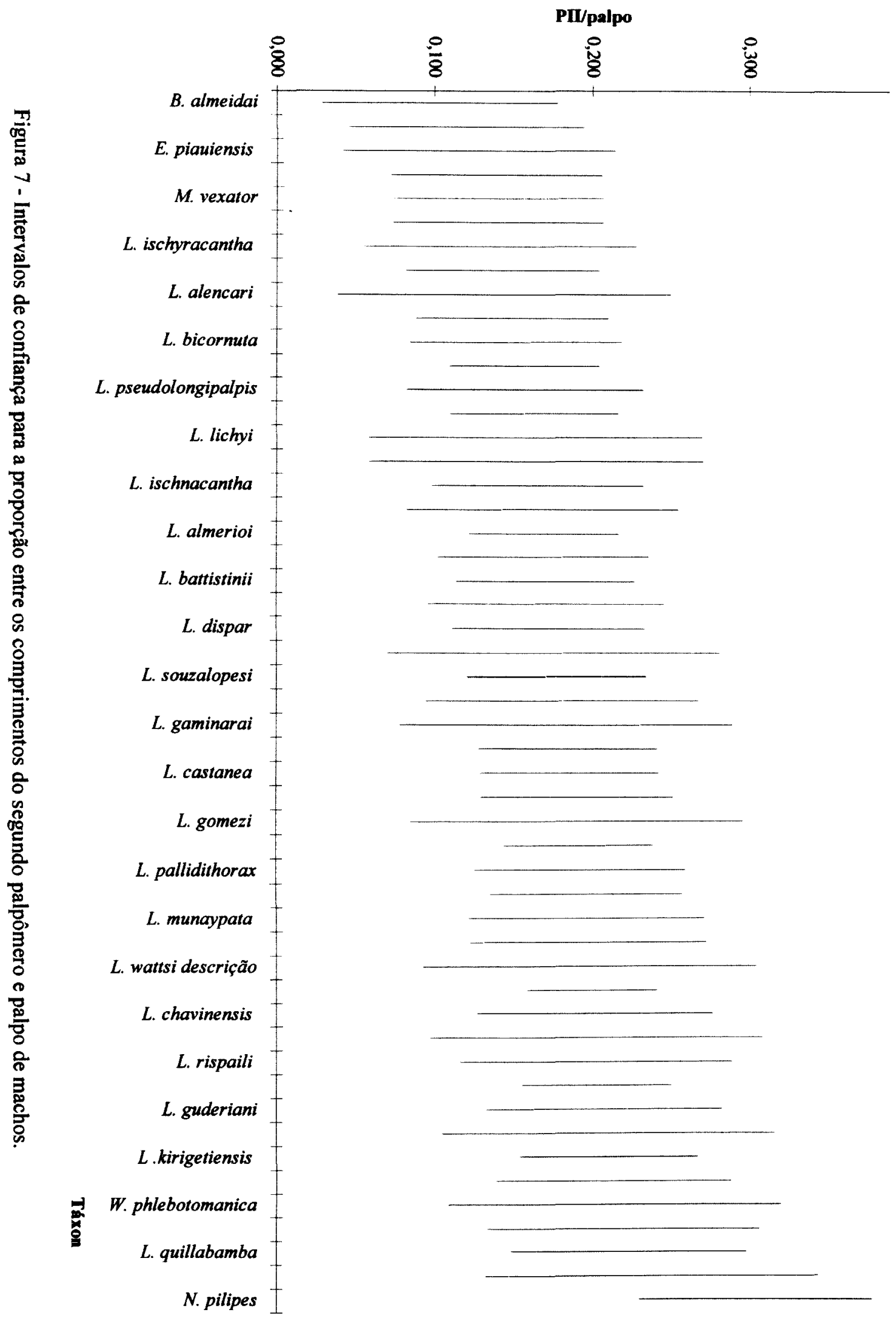




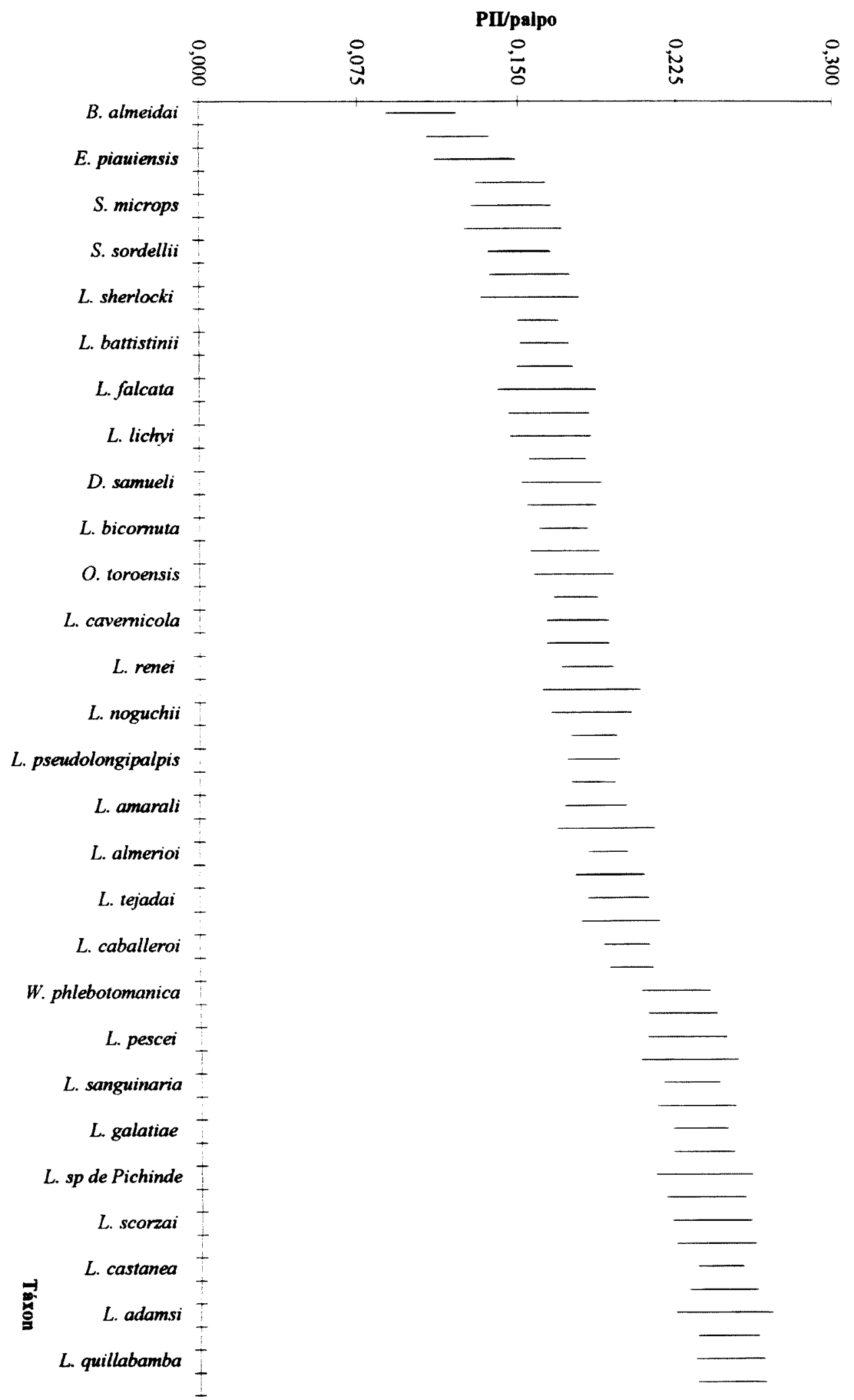




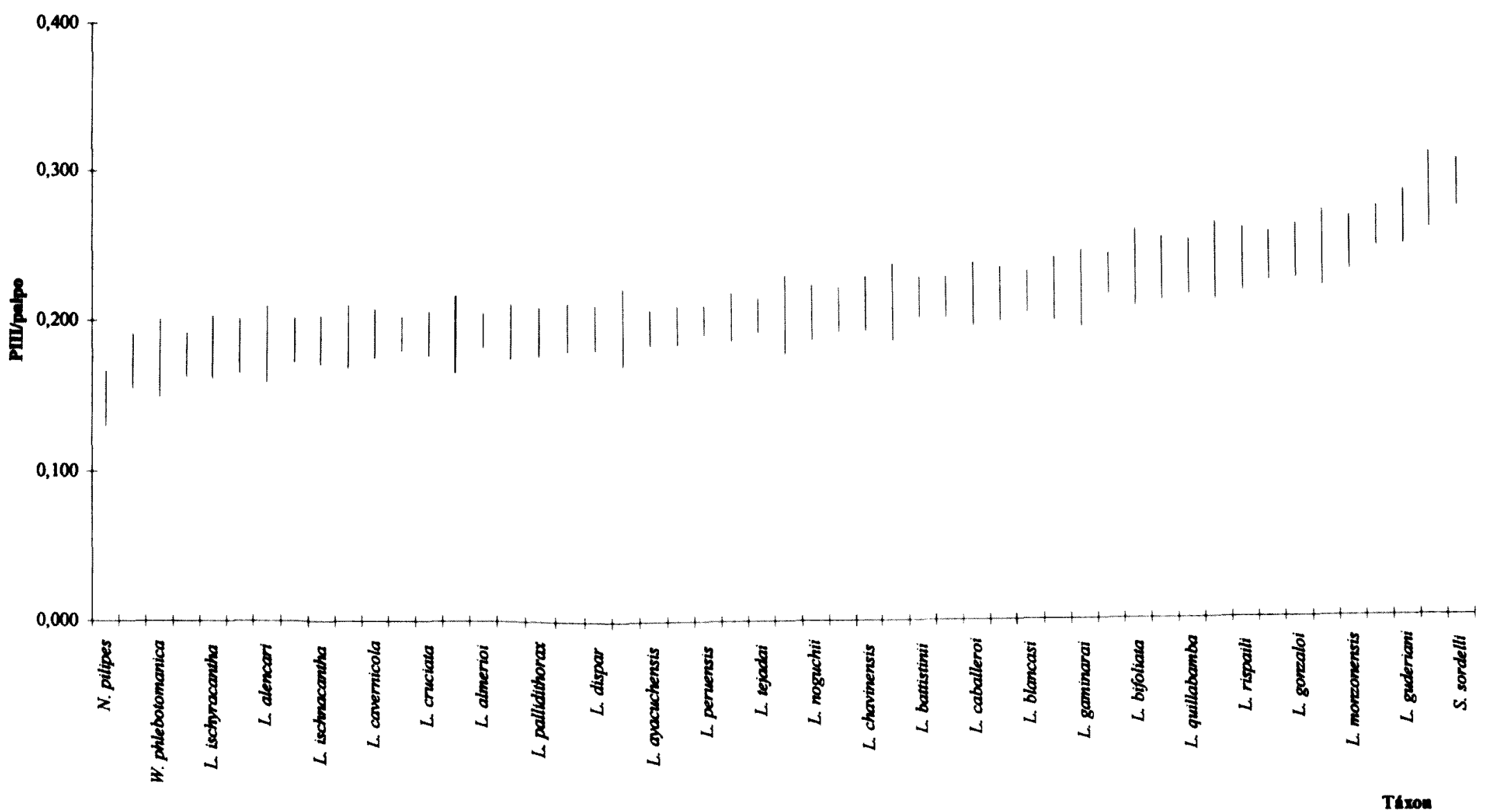

Figura 9 - Intervalos de confiança para a proporção entre os comprimentos do terceiro palpômero e palpo de machos. 


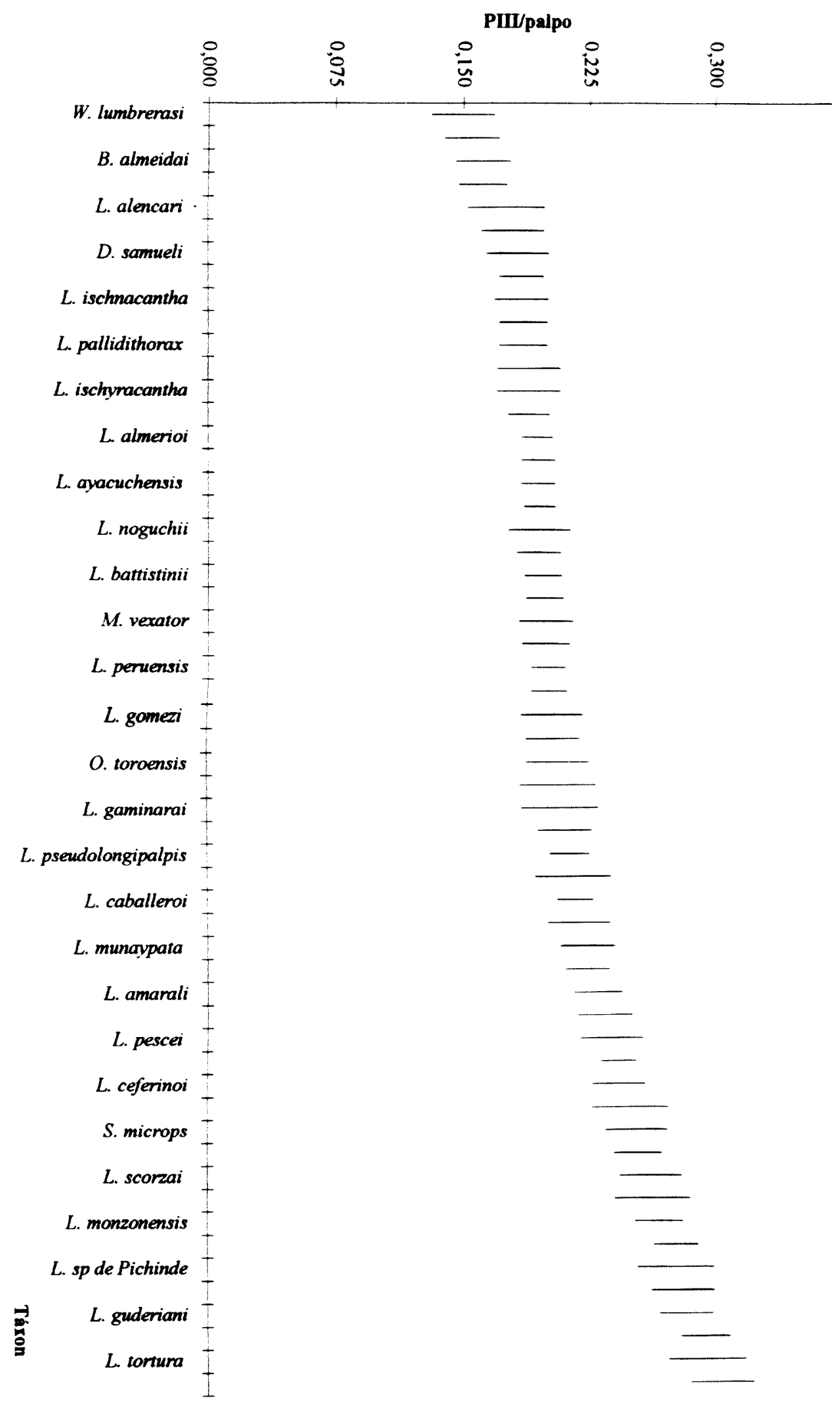




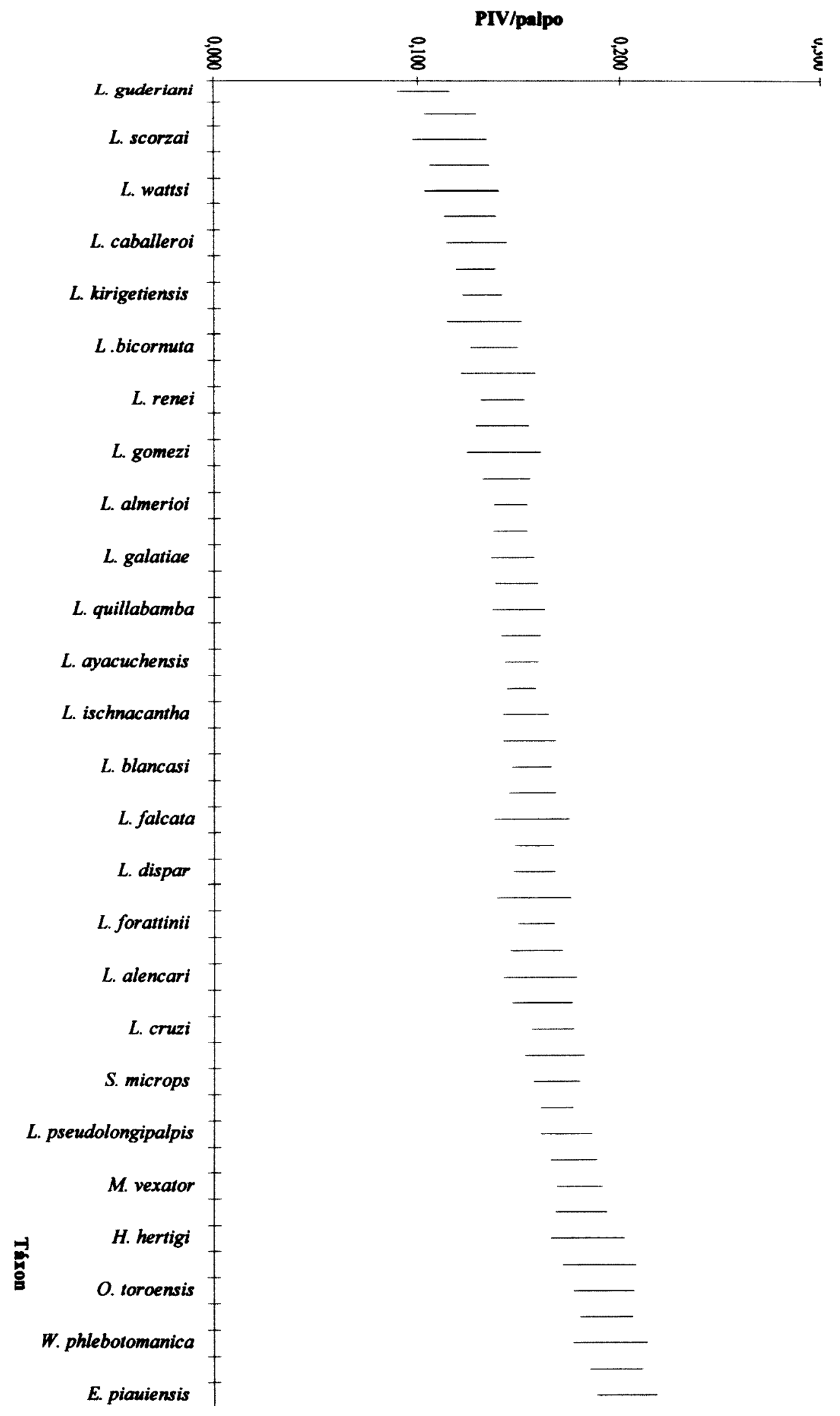




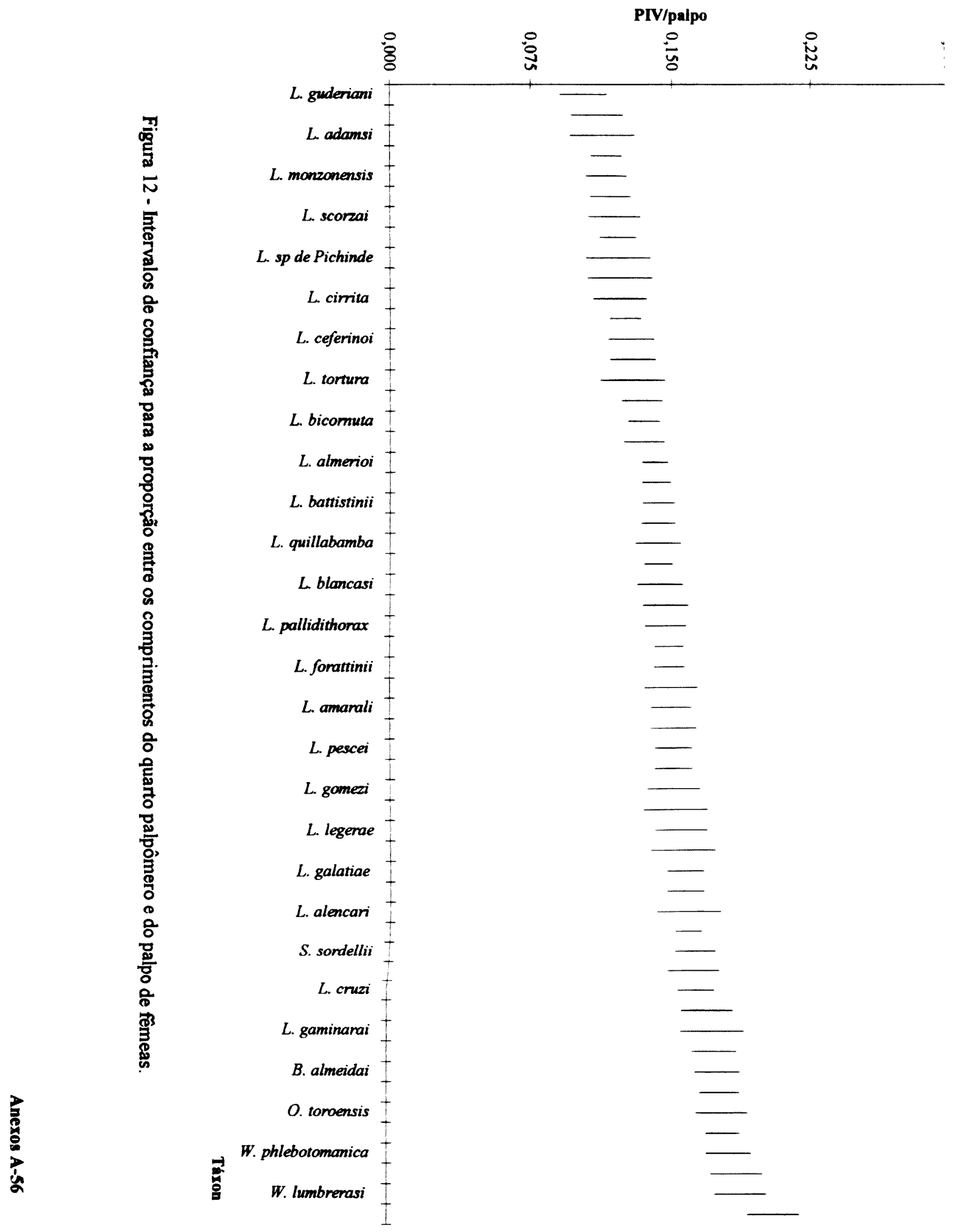




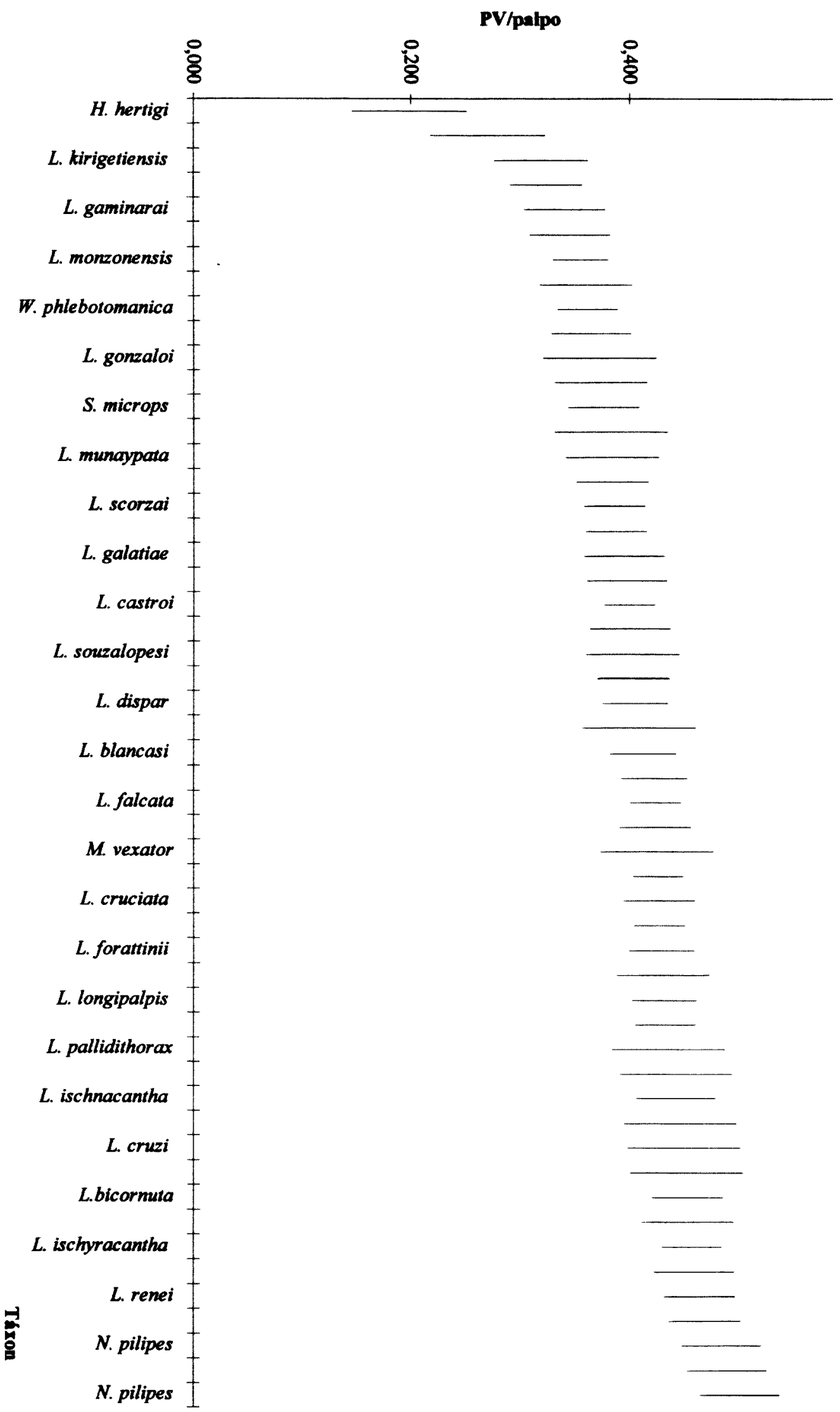



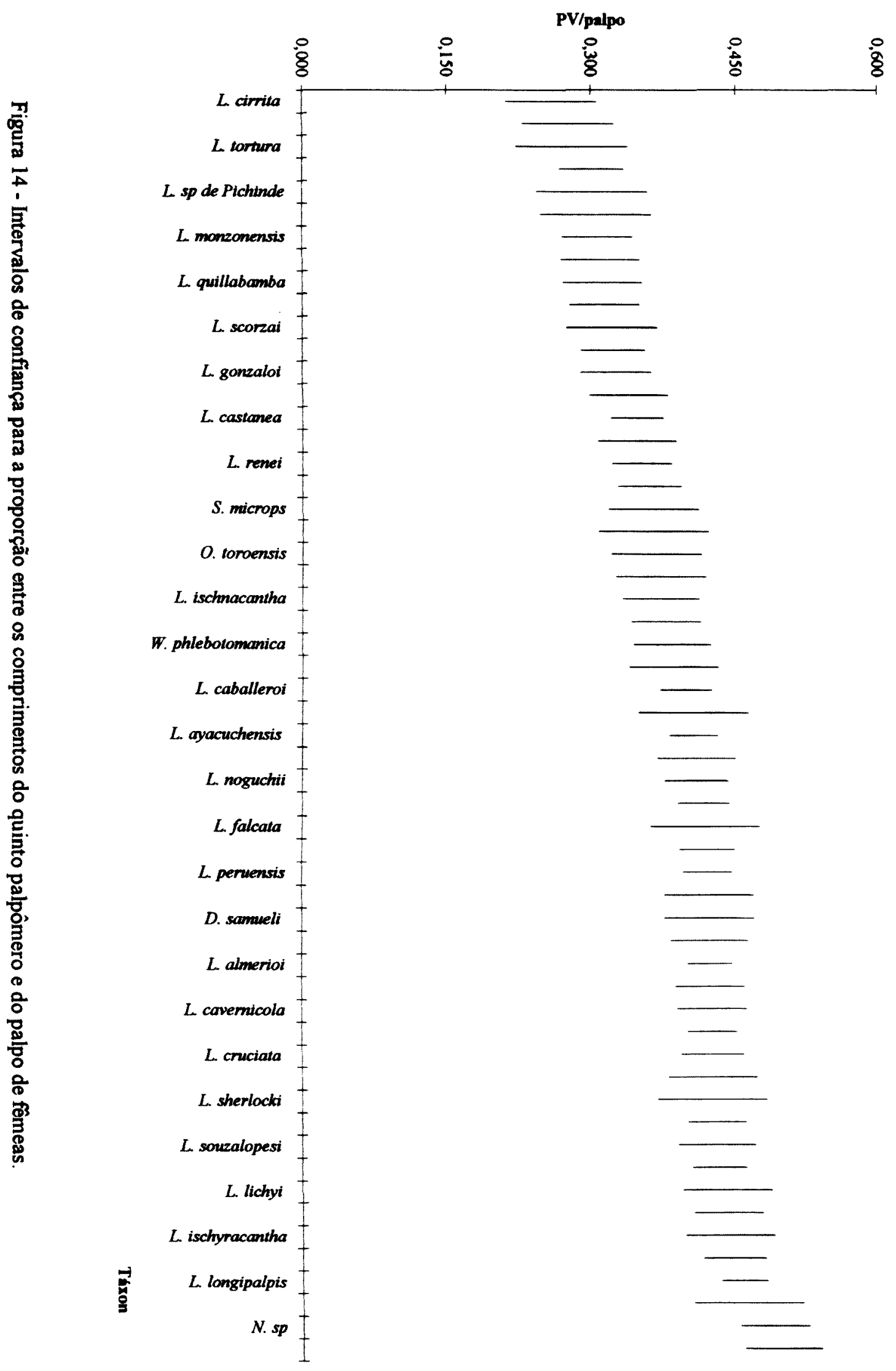


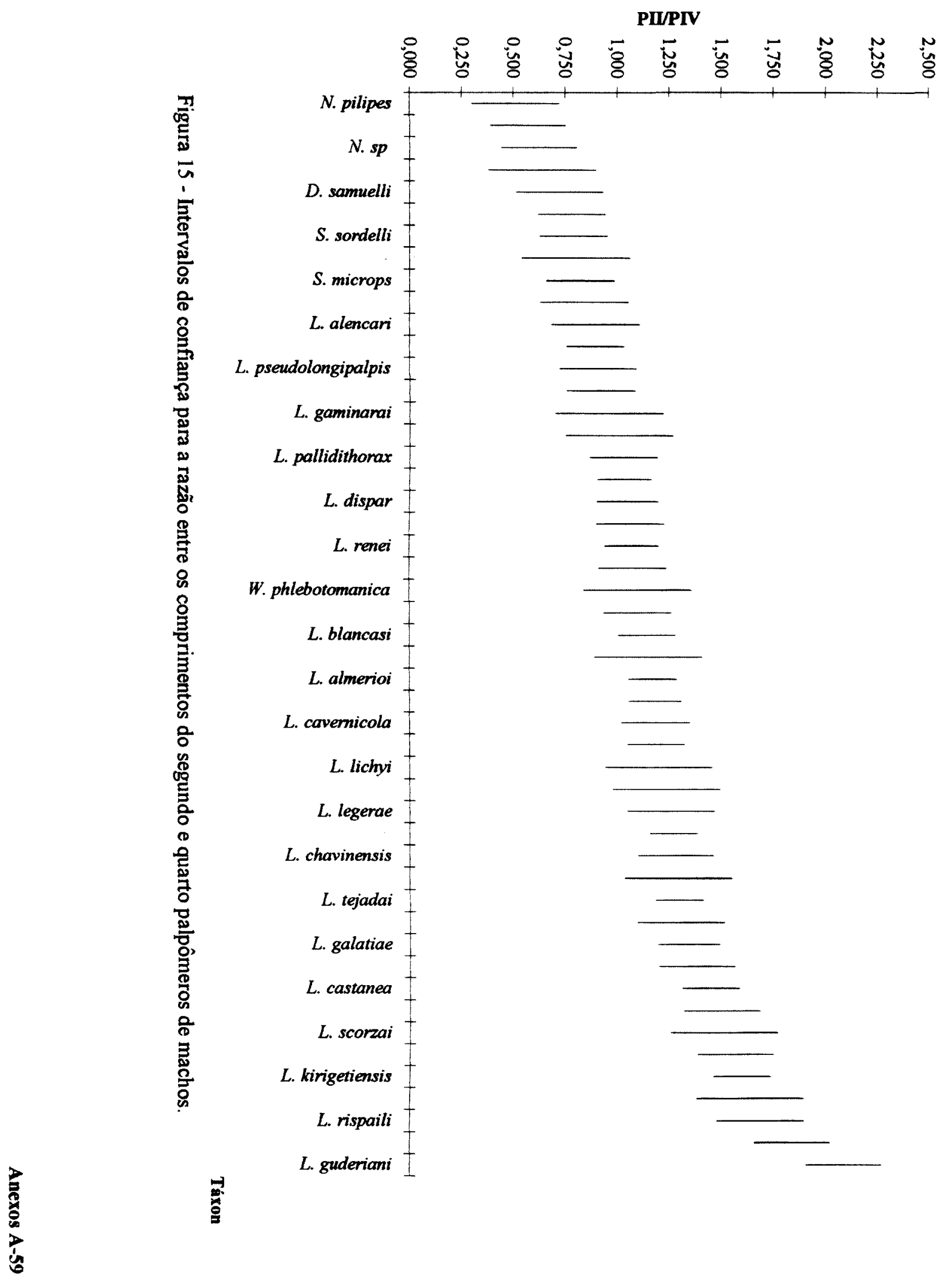




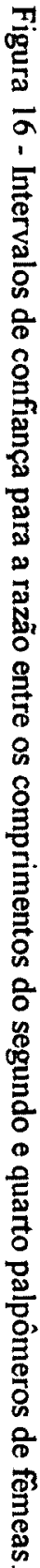

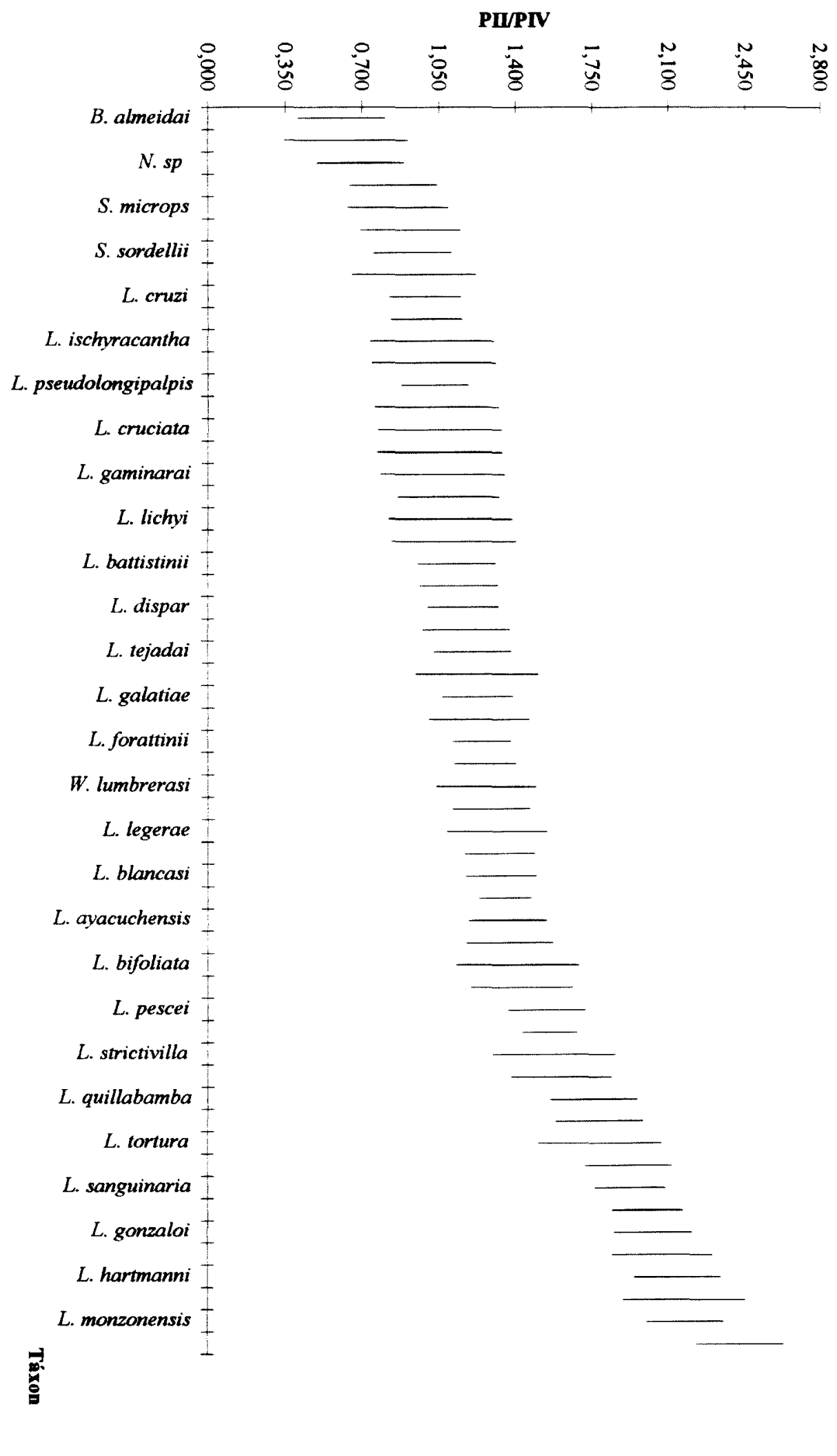




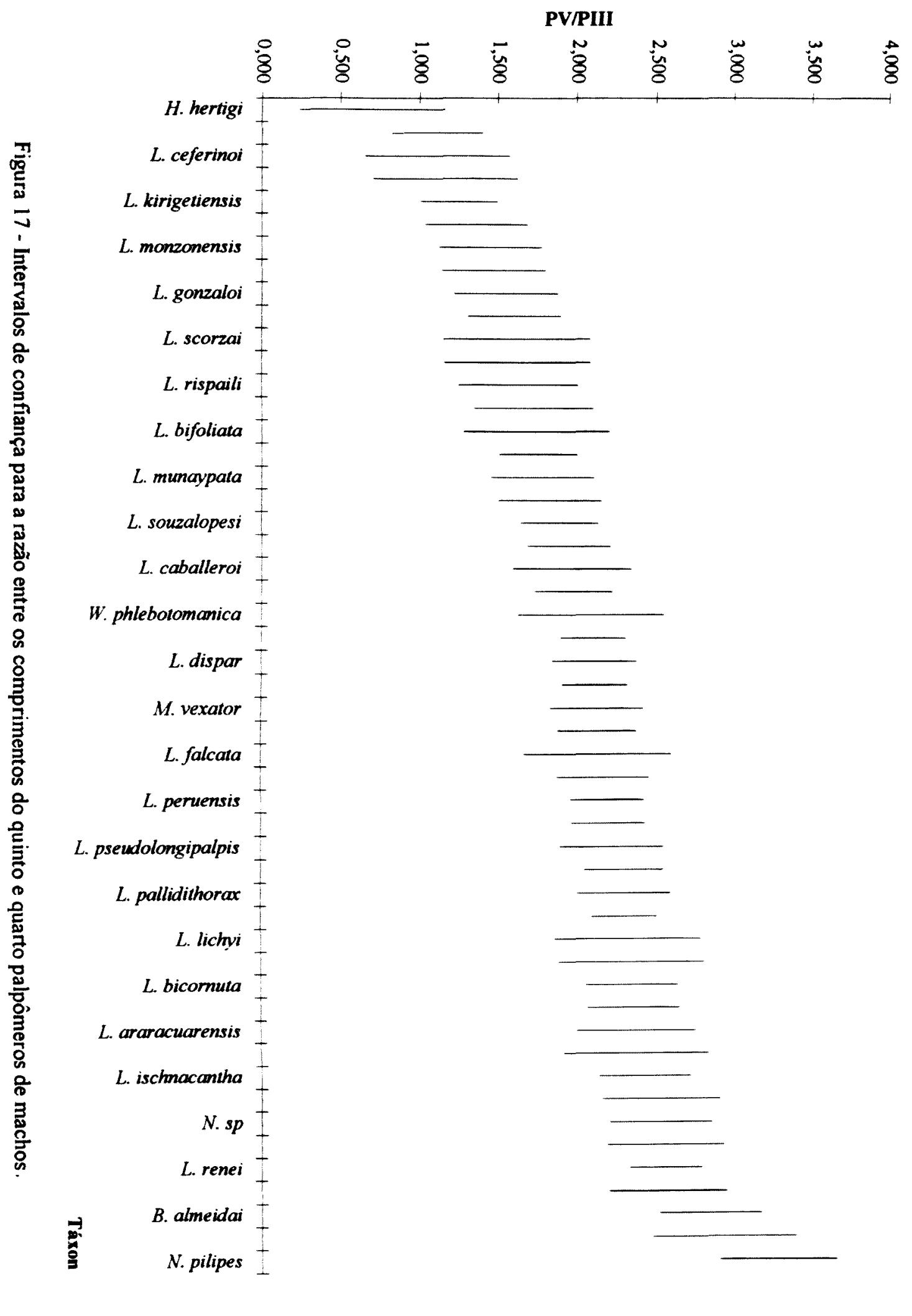




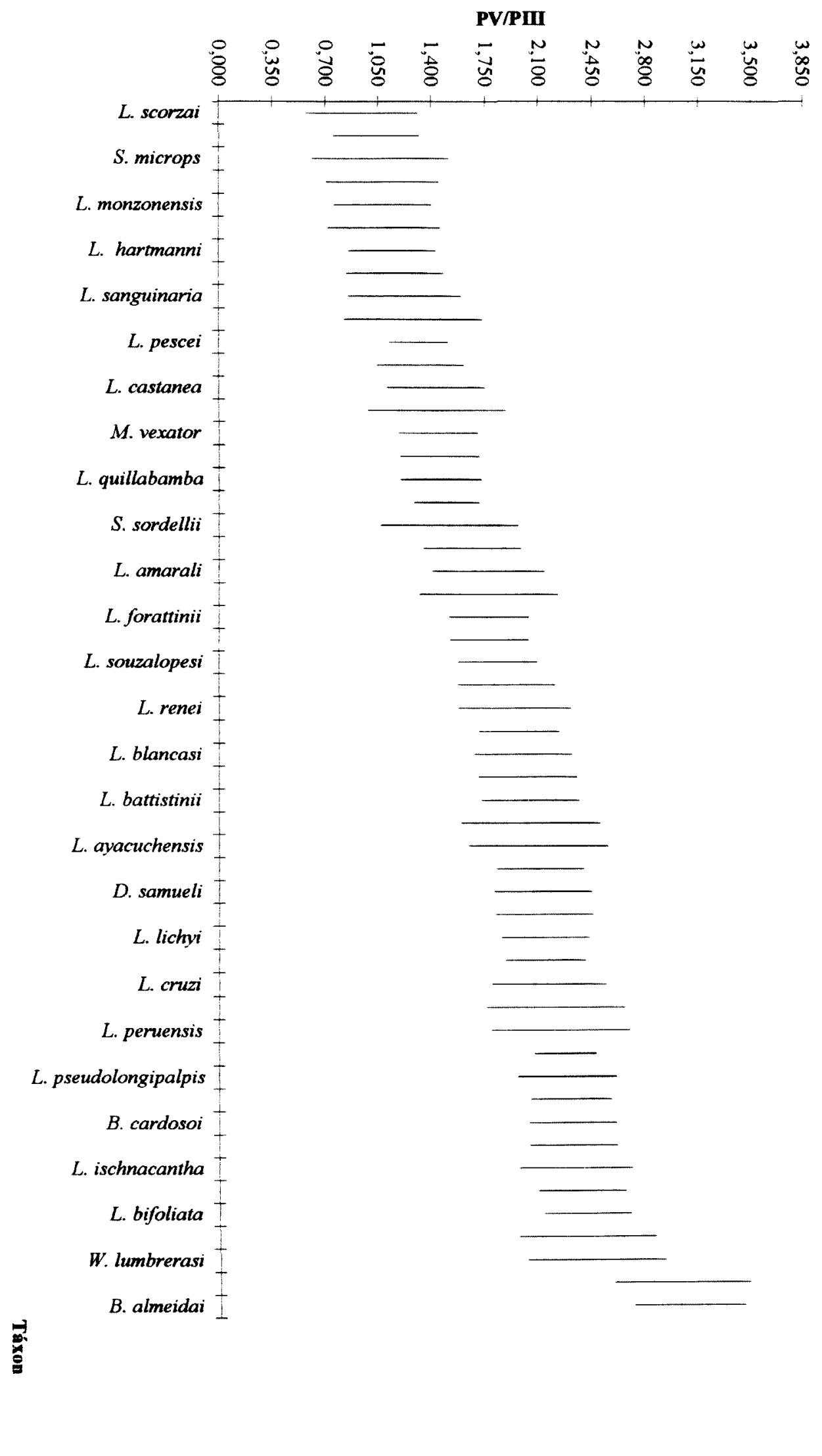




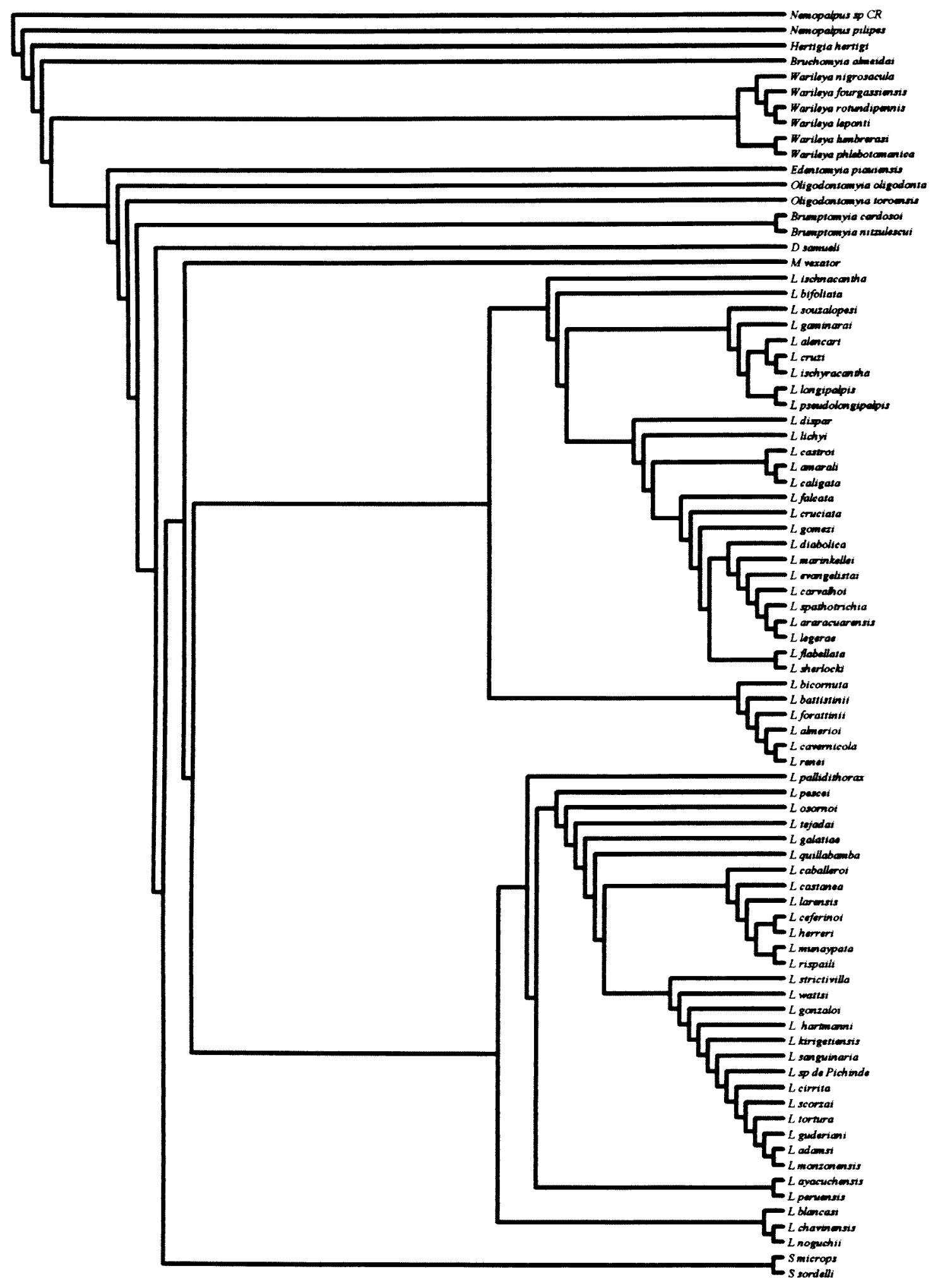

Figura 19A - Cladograma de consenso semi-estrito ( $I C=0,378, I R=0,84)$ do gênero Lutzomyia As figuras subseqüentes mostram detalhes deste cladograma: 19B = gênero Lutzomyia; 19C = subgênero Lutzomyia s. str. e 19D subgênero Helcocyrtomyia. 


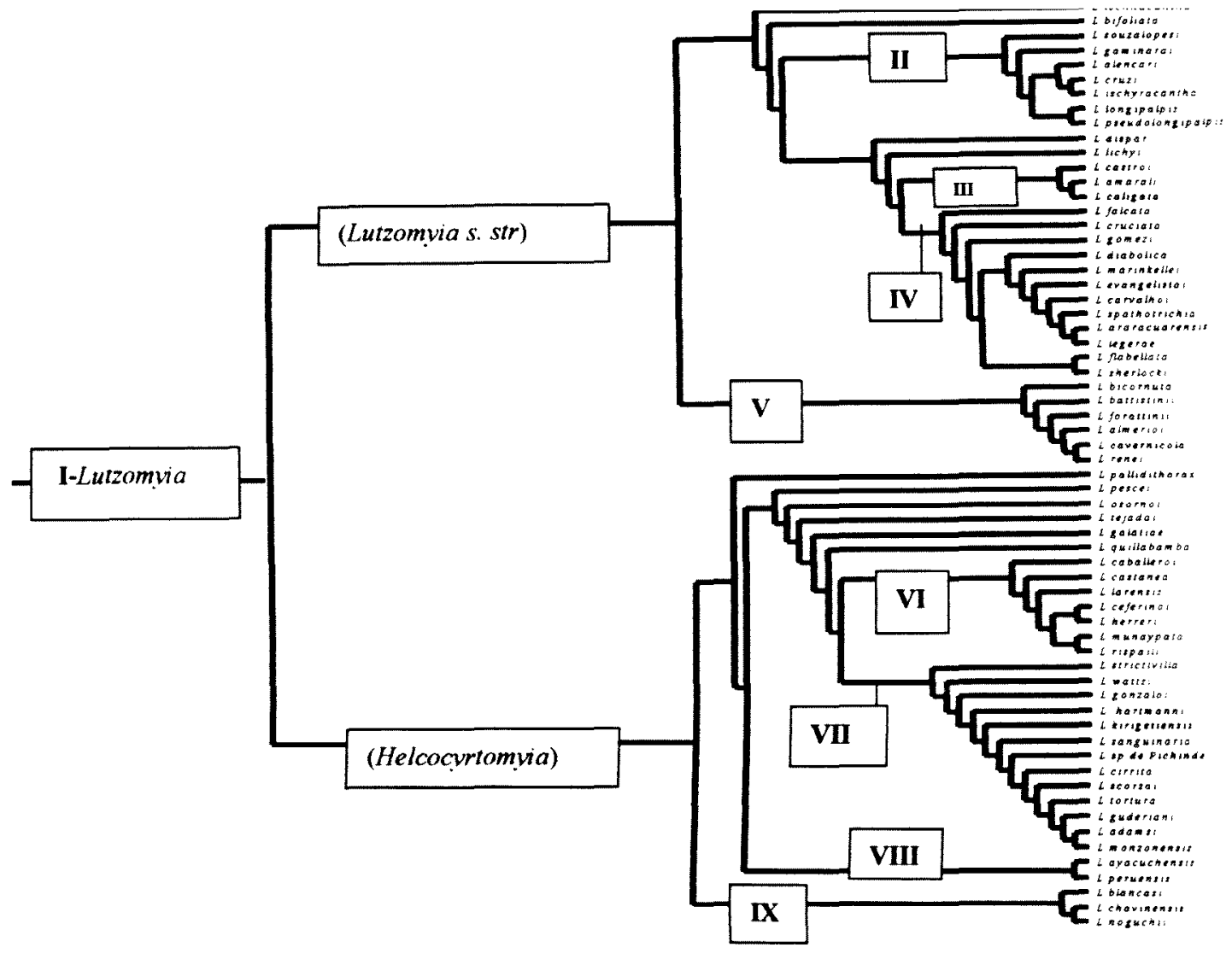

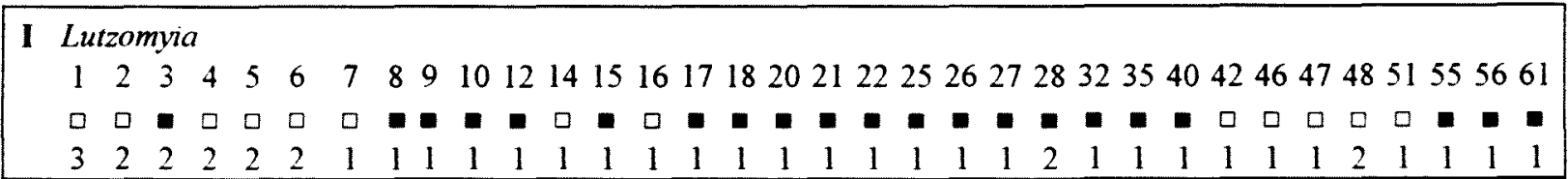

Figura 19B - Cladograma representativo do gênero Lutzomvia e seus grupos monofiléticos propostos como subgêneros Lutzomvia $s$. str. e Helcocyrtomyia e suas respectivas séries de espécies. $\mathbf{O}$ quadro 1 mostra as sinapomorfias do gênero em questão; II = série longipalpis; III = série castroi $; \mathbf{I V}=$ série cruciata; $\mathbf{V}=$ série cavernicola; $\mathbf{V I}=$ série caballeroi; $\mathbf{V I I}=$ série sanguinaria; $\mathbf{V I I I}=$ série peruensis; $\mathbf{I X}=$ série noguchii. $\mathbf{\square}=$ sinapomorfia; $\square=$ homoplasia ou reversão; número acima do quadrado indica o caráter; número abaixo do 
Figura 19C - Cladograma de Lutzomvia s. str. $\mathbf{\square}=$ sinapomorfia; $\square=$ homoplasia ou reversão: número acima do quadrado indica o caráter: número abaixo do quadrado indica estado do caráter, quando acompanhado de $\mathrm{R}$ significa reversão para o caráter. 


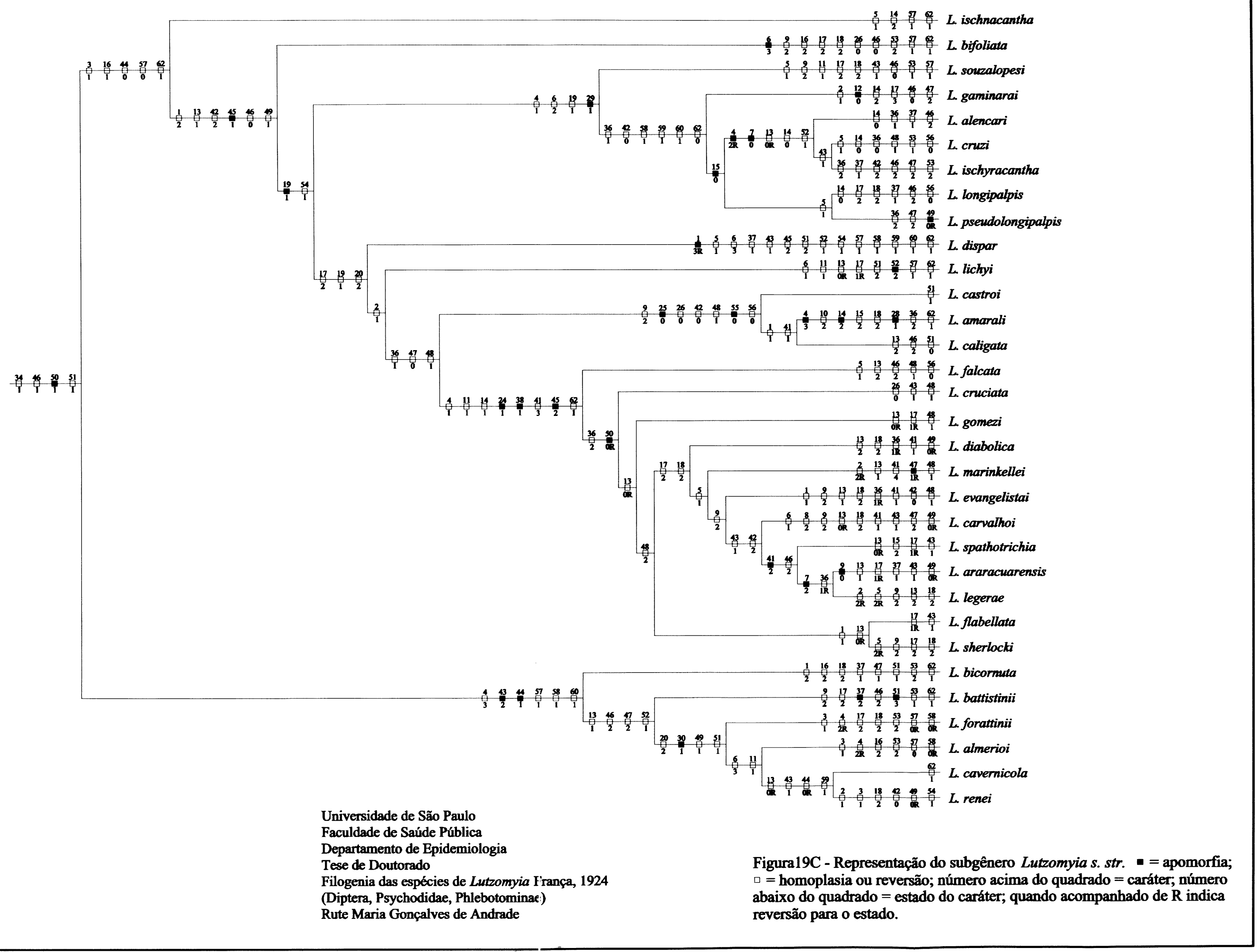


Figura 19D - Cladograma do subgênero Helcocyrtomyia $\mathbf{a}=$ sinapomorfia; $\square=$ homoplasia ou reversão: número acima do quadrado indica o caráter; número abaixo do quadrado indica estado do caráter, quando acompanhado de $\mathbf{R}$ significa reversão para o caráter. 


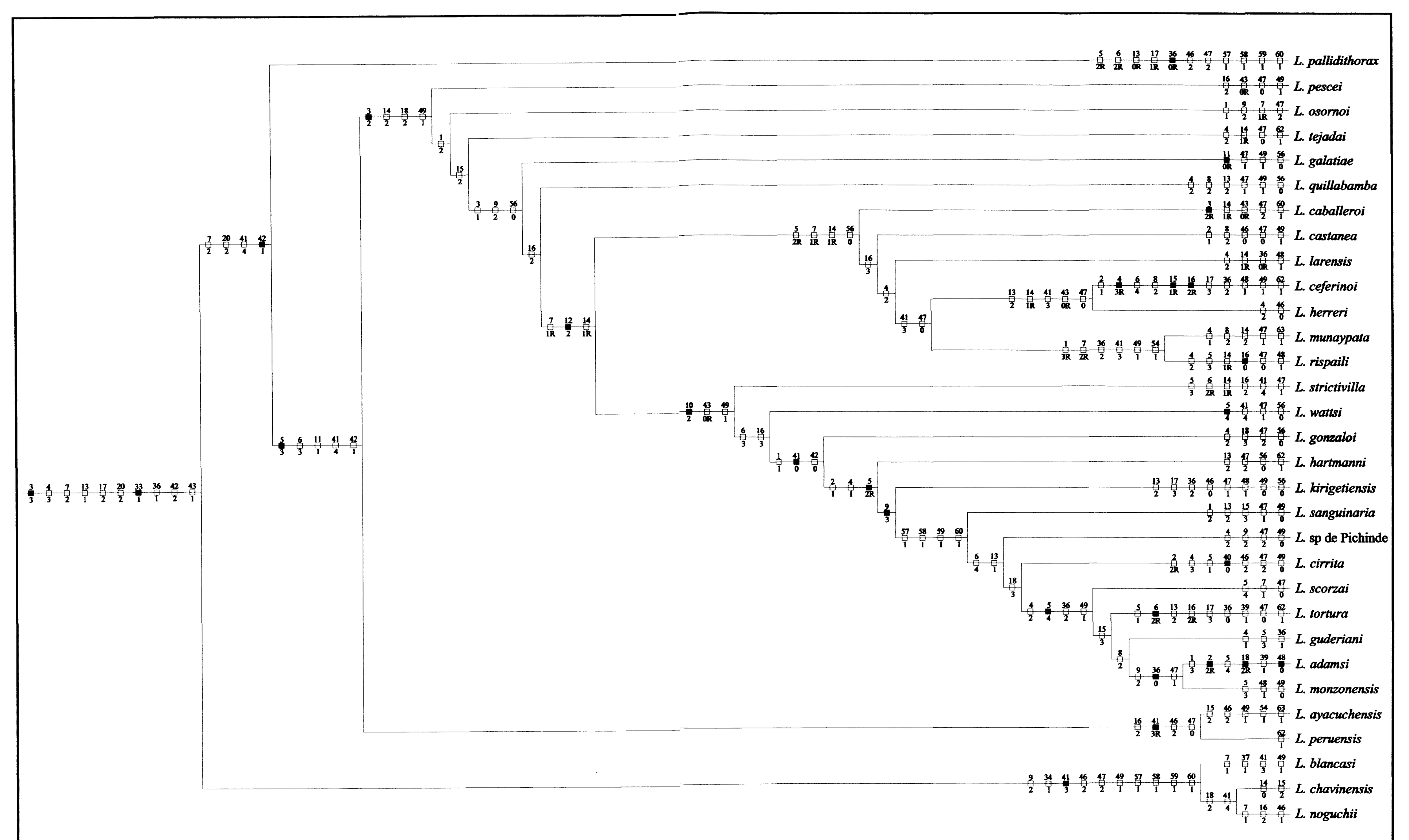




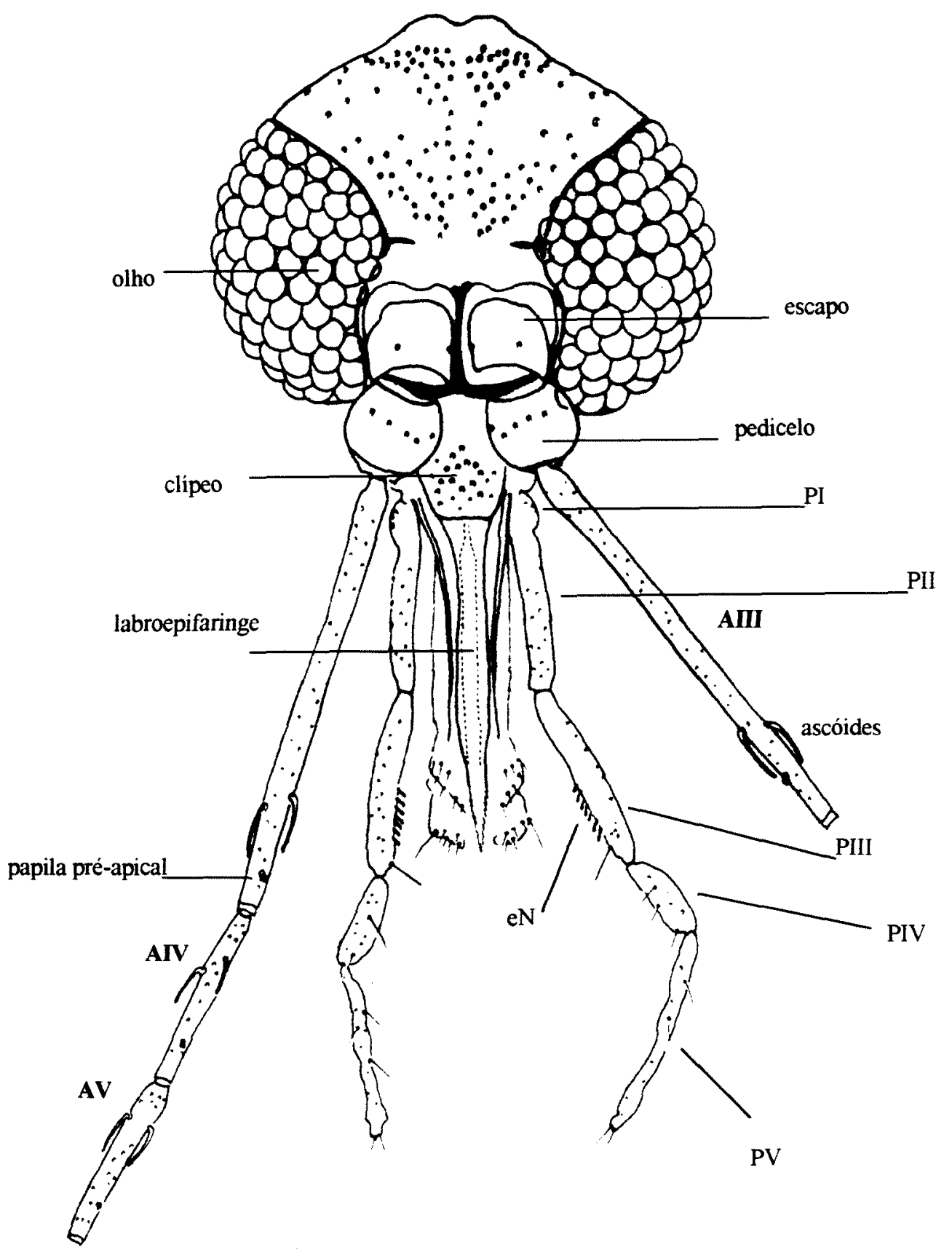

Figura 20 - Desenho esquemático de cabeça de Phlebotominae. Segmentos da antena: escapo, pedicelo, AIIl (terceiro segmento), AIV (quarto) e AV (quinto); eN: espinhos de Newstead; PI-PV: primeiro ao quinto palpômeros. 

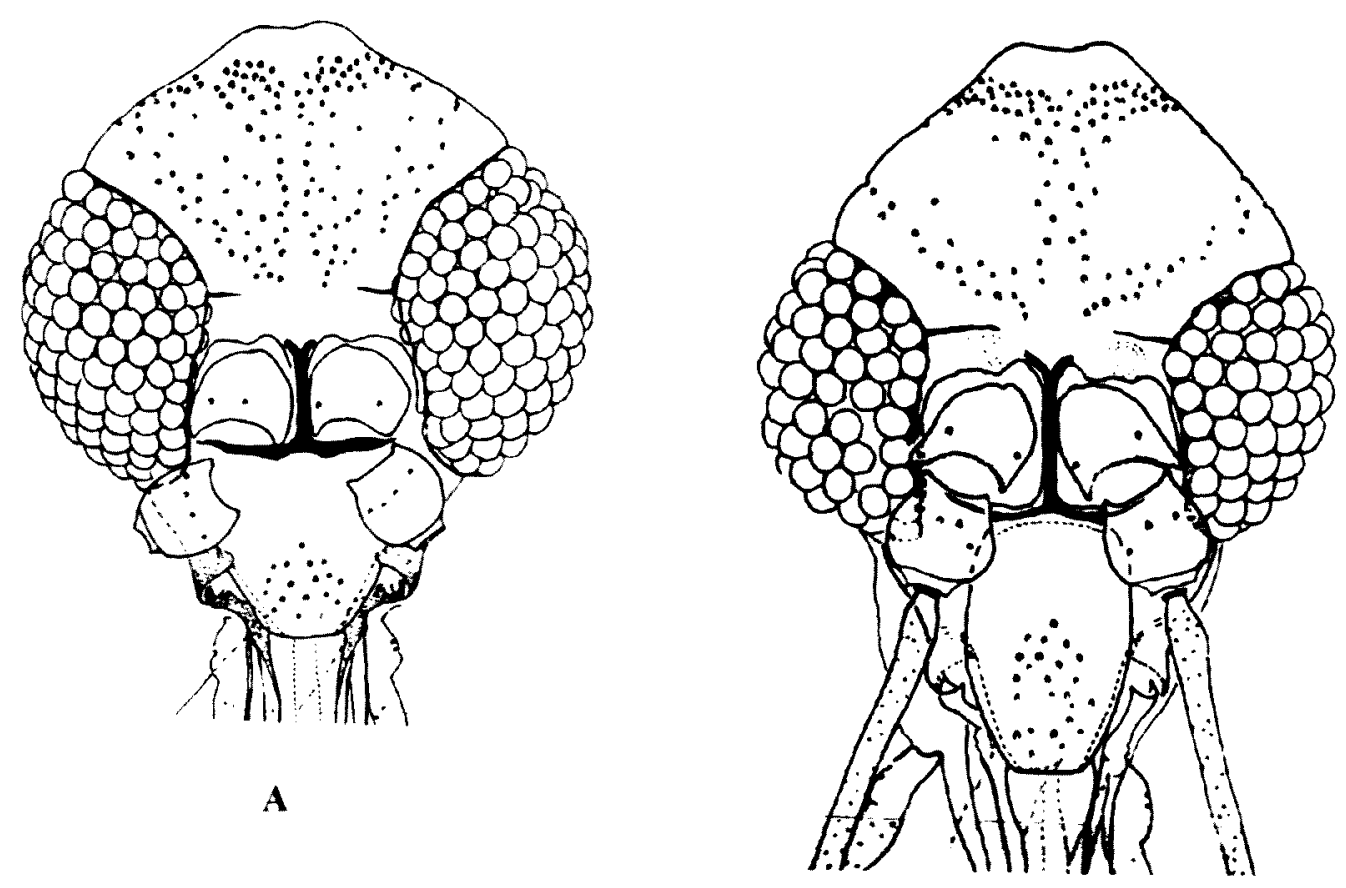

B

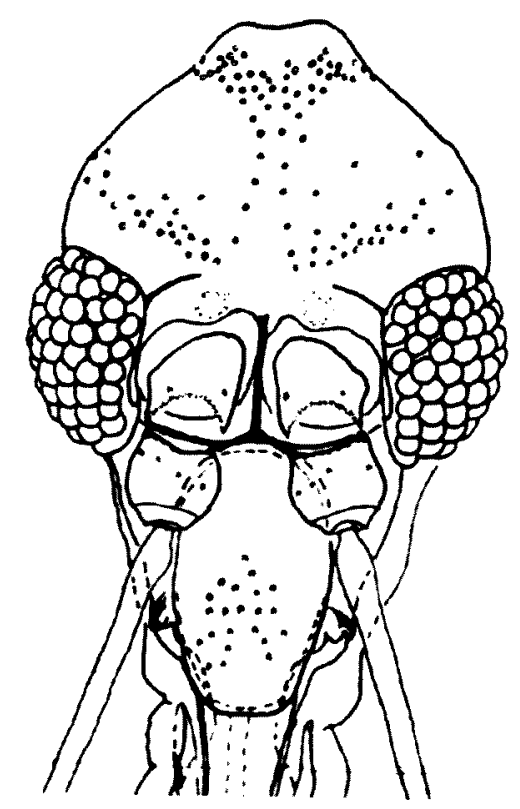

A

Figura 21 - Cabeças de especies de Lutzomvia (Helcocyrtomvia) com diferentes comprimentos do clipeo e olhos em relação à cabeça. $\mathbf{A}=L$. (H.) kirigetiensis (Adaptado de Galati \& Cáceres. 1992) com clipeo curto e olhos grandes: $\mathbf{B}=L$. (H.) pallidithorax (Adaptado de Galati \& Cáceres. 1992) com clipeo e olhos medianos: $\mathbf{C}=L$. $(H$. blancasi (Adaptado de Galati \& Cáceres. 1990) com clipeo longo e olhos pequenos. 


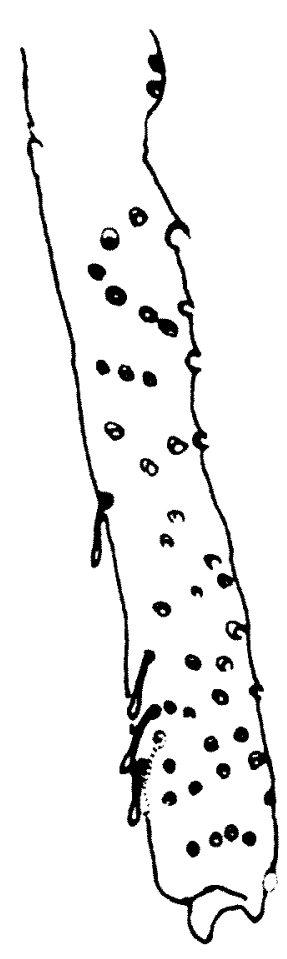

A

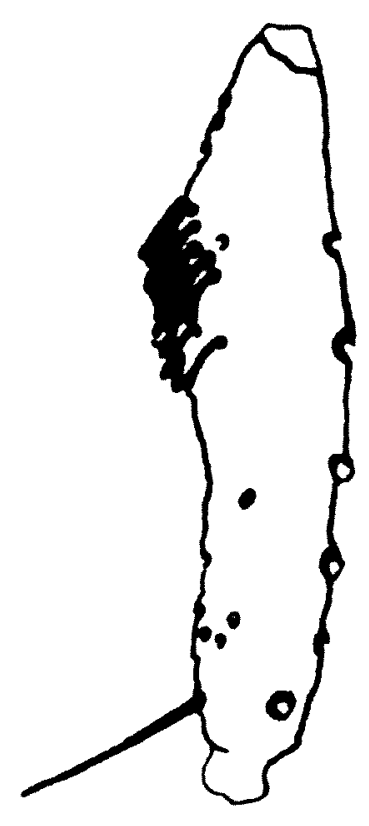

B
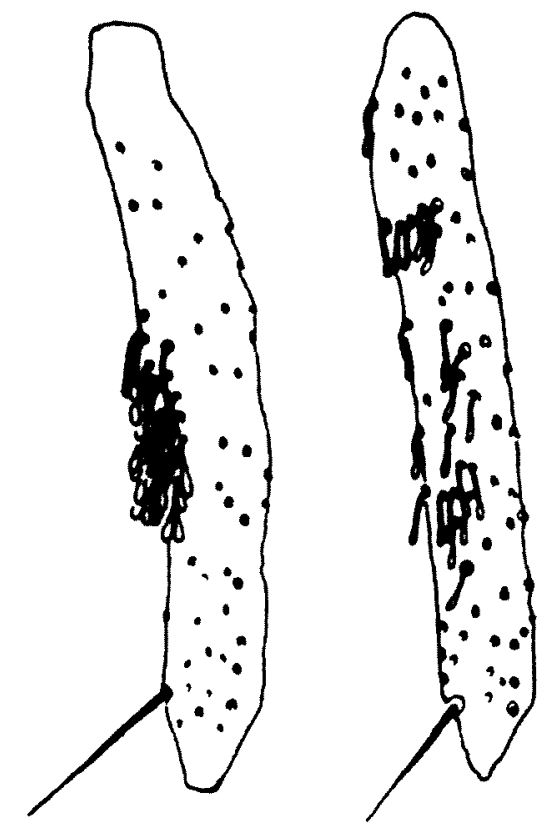

C
D

Figura 22 - Palpômeros de Phlebotominae. $\mathbf{A}=$ primeiro e segundo palpòmeros de fêmea de $L$. cruciata com espinhos de Newstead $(\mathrm{eN})$ presentes no segundo segmento do palpo; $\mathbf{B}=$ fêmea de Edentomvia piauiensis com os $\mathrm{eN}$ no terço basal; $\mathbf{C}=\mathrm{eN}$ na regiào mediana: $\mathbf{D}=$ fềmea de $I$. pallidithorax com $\mathrm{eN}$ dispersos pelo segmento palpal. 


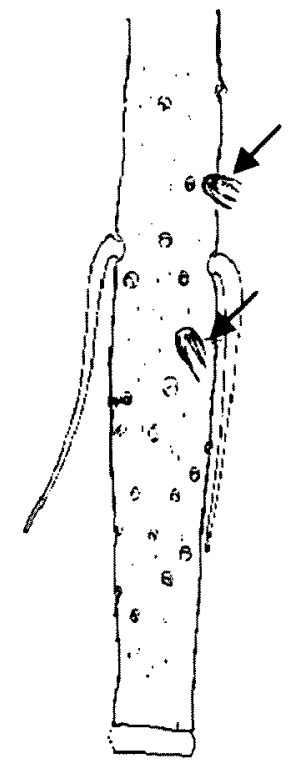

A

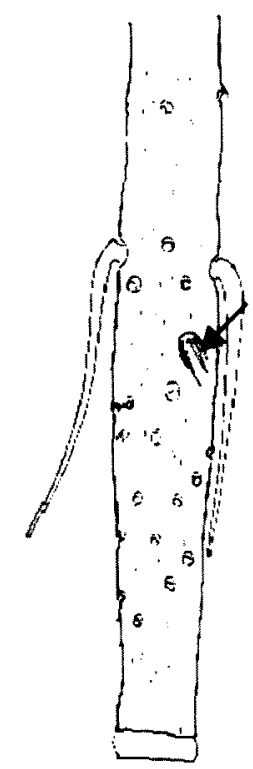

B

Figura 23 - Terceiro segmento da antena de Phlebotominae. $A=$ presença das papilas pré-ascoidal e pré-apical; $\mathrm{B}=$ presença de papila pré-apical. 


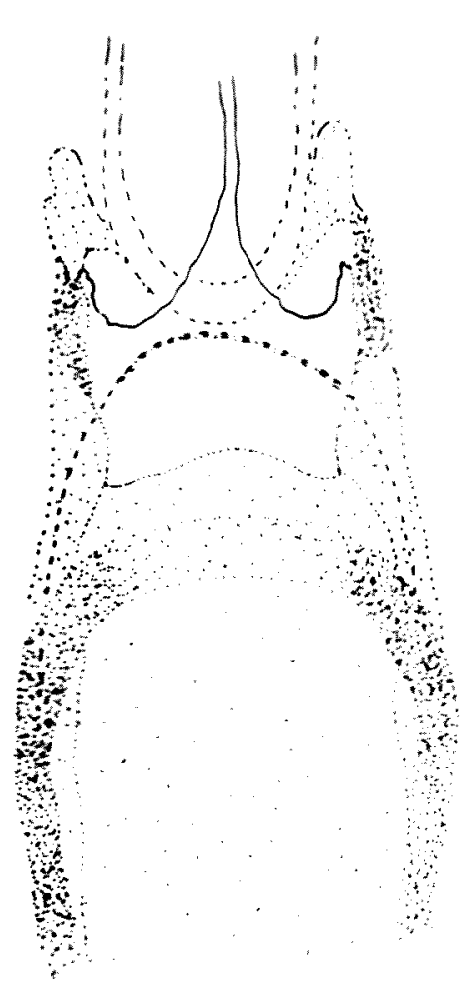

A

D

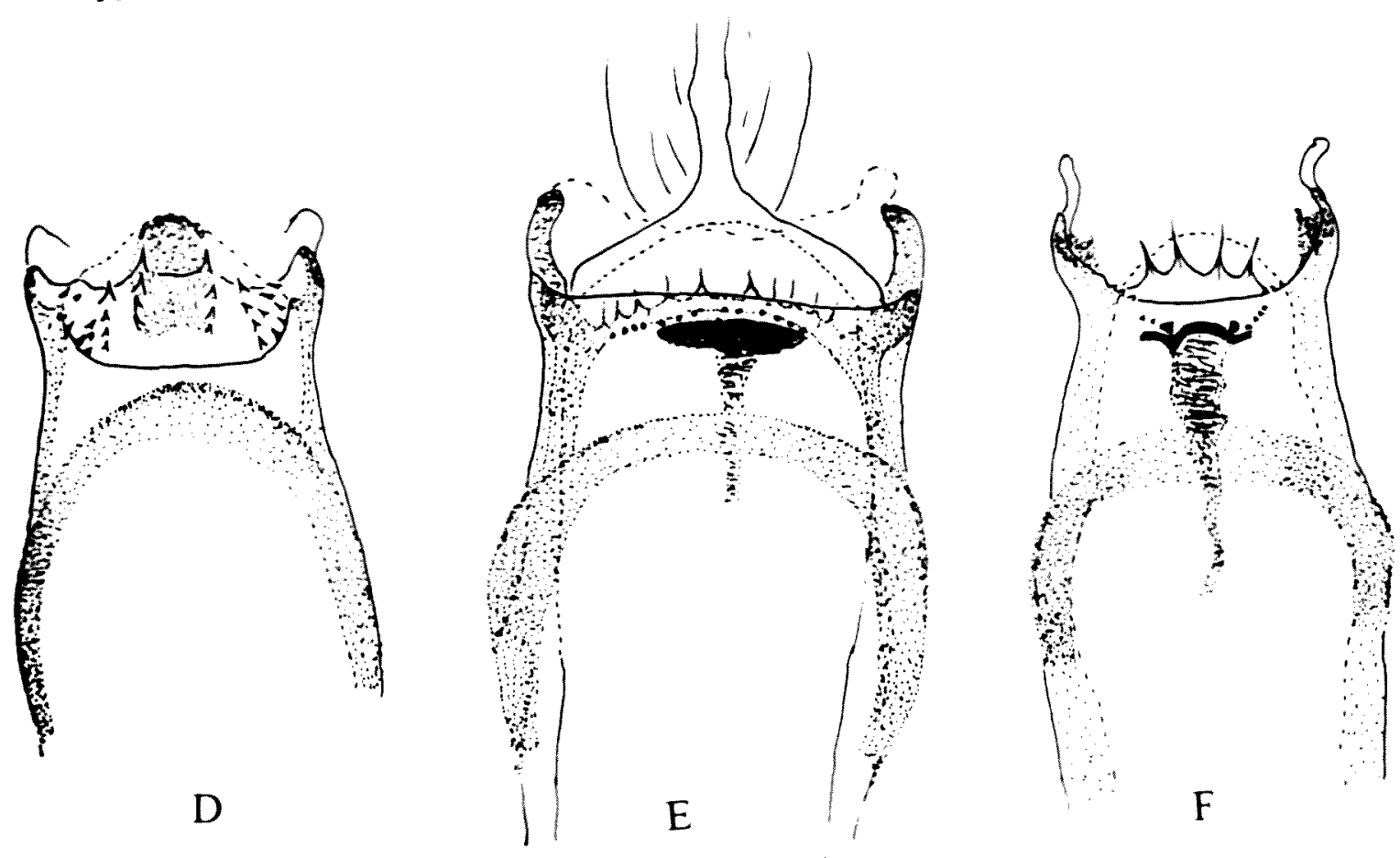

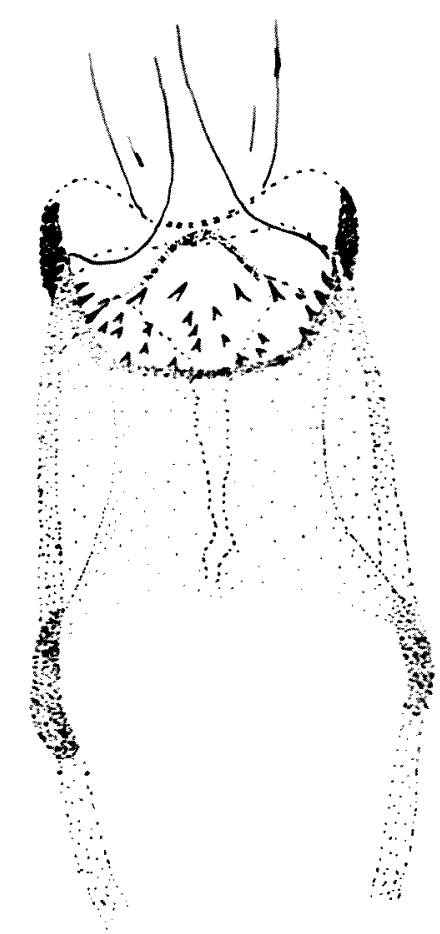

B

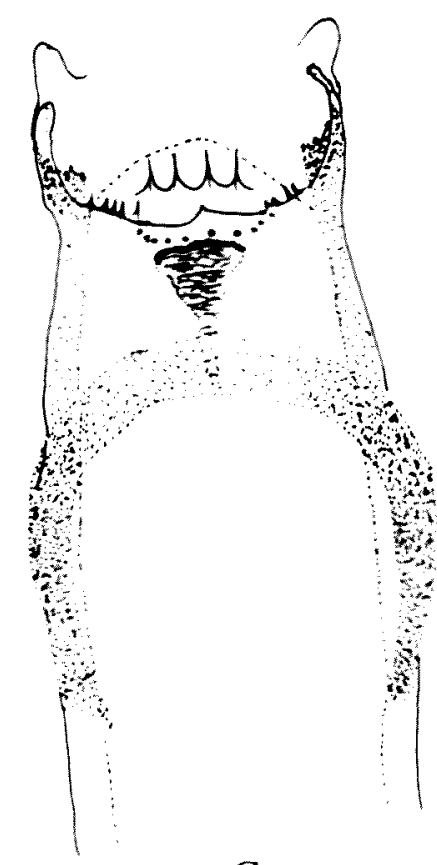

C

Figura 24 - Cibários de Phlebotominae. $\mathrm{A}=\boldsymbol{W}$. phlebotomanica; $\mathbf{B}=$ Lutzomyia (Castromyia) $\mathrm{sp}$; $\mathrm{C}=L$. battistinii $; \mathrm{D}=$ Brumptomvia $\mathrm{sp} ; \mathrm{E}=$ L. longipalpis $; \mathrm{F}=L$. forattinit 


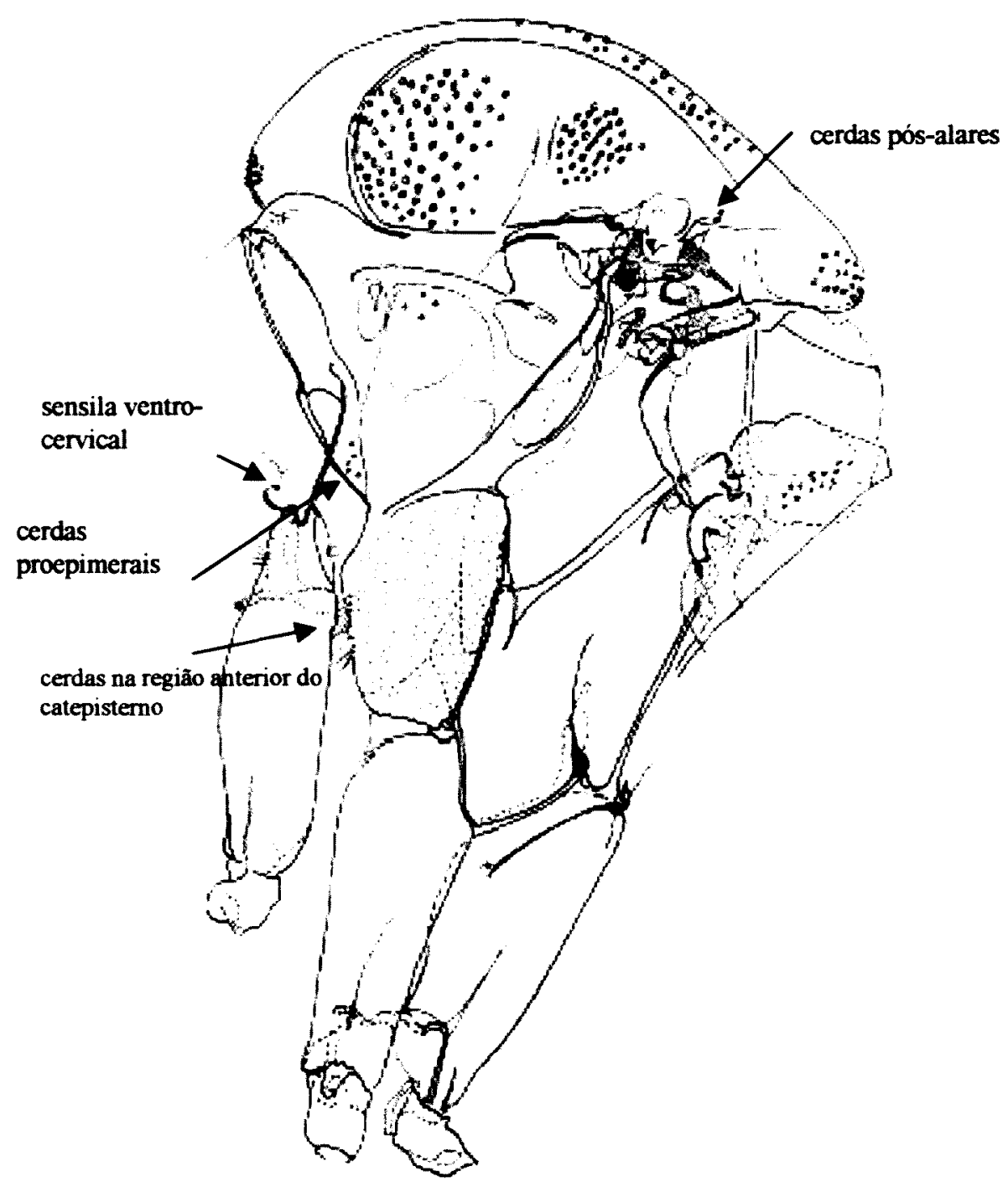

Figura 25 - Tórax de Edentomyia piauiensis com as cerdas pós-alares, catepisternais, proepimerais e sensila ventro-cervical presentes (Adaptação de Galati e col. 2003). 


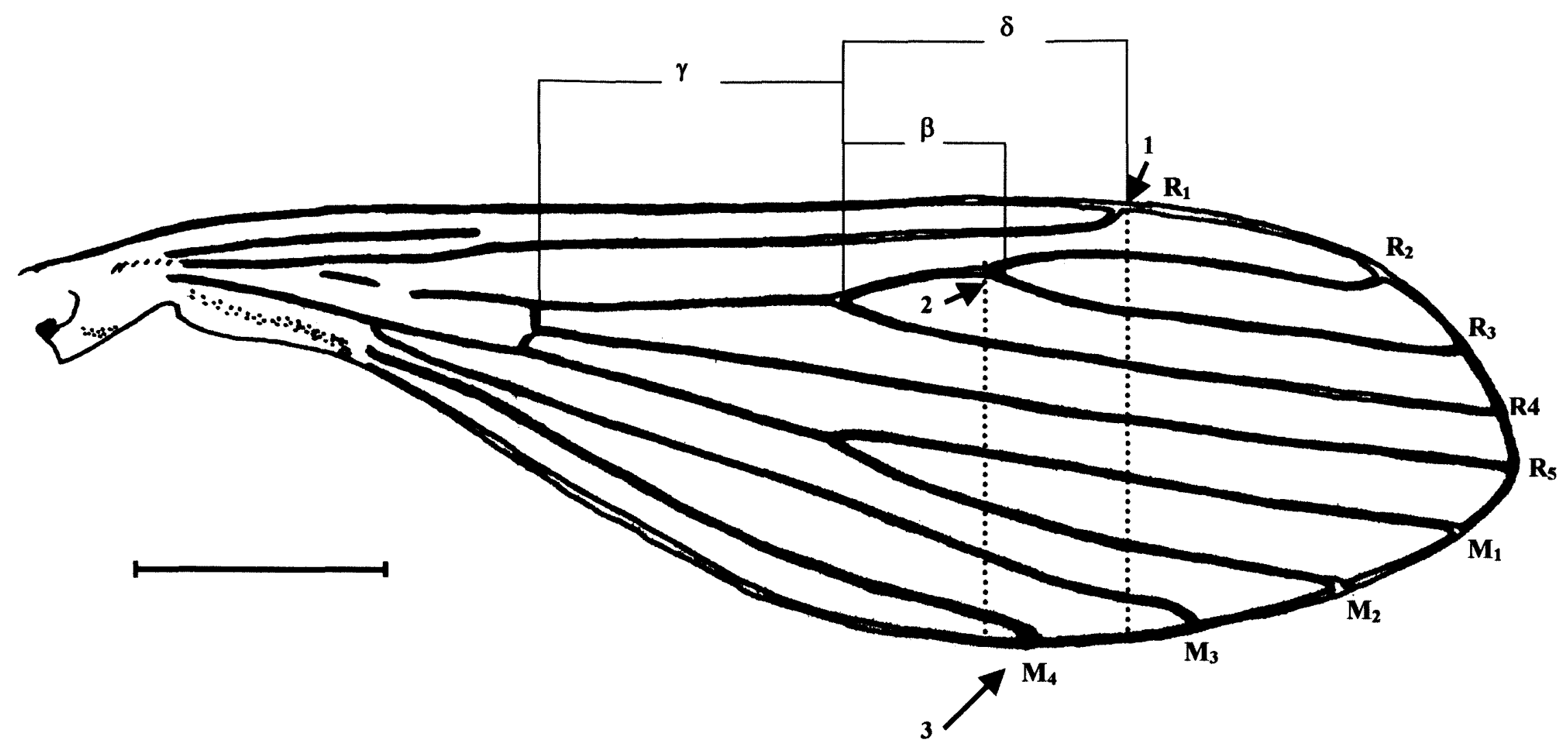

Figura 26 - Asa de Phlebotominae. Os tracejados correspondem aos segmentos observados nos caracteres 36 e 37: início da veia $R_{1}$ (seta 1) e junção das nervuras $R_{2}$ e $R_{3}$ (seta 2) em relação ao início da nervura $\mathrm{M}_{4}$ (seta 3). Nesta asa o início de $R_{1}$ está mais distal que o início de $\mathbf{M}_{4}$, o que corresponde ao estado apomórfico para este caráter e, a junção de $R_{2}$ e $R_{3}$ está mais proximal que o início de $\mathbf{M}_{4}$, o que corresponde ao estado plesiomórfico do caráter. Escala $=100 \mu \mathrm{m}$ 


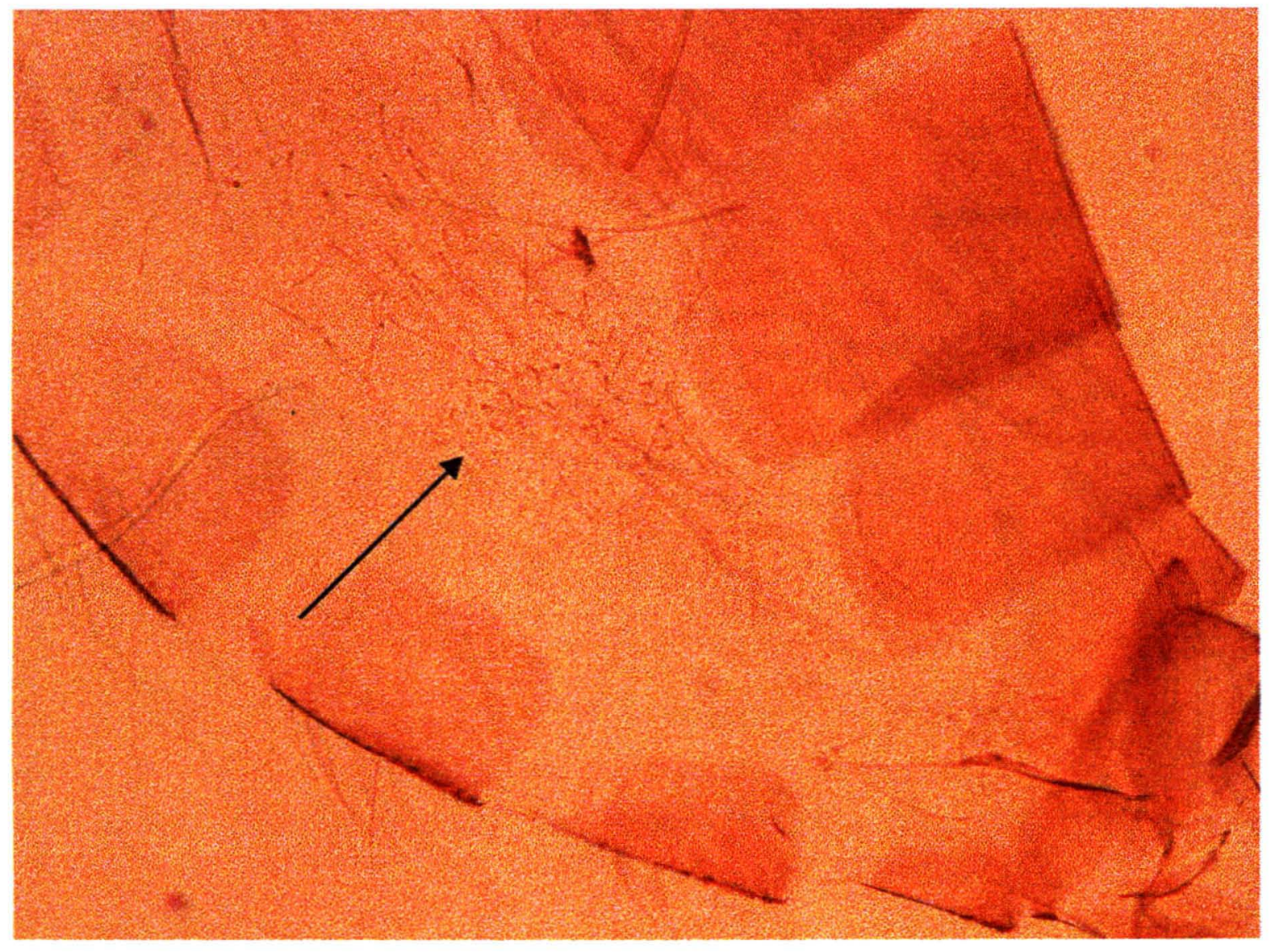

Figura 27 - Cerdas nas pleuras abdominais de fềmea de Lutzomyia falcata. 


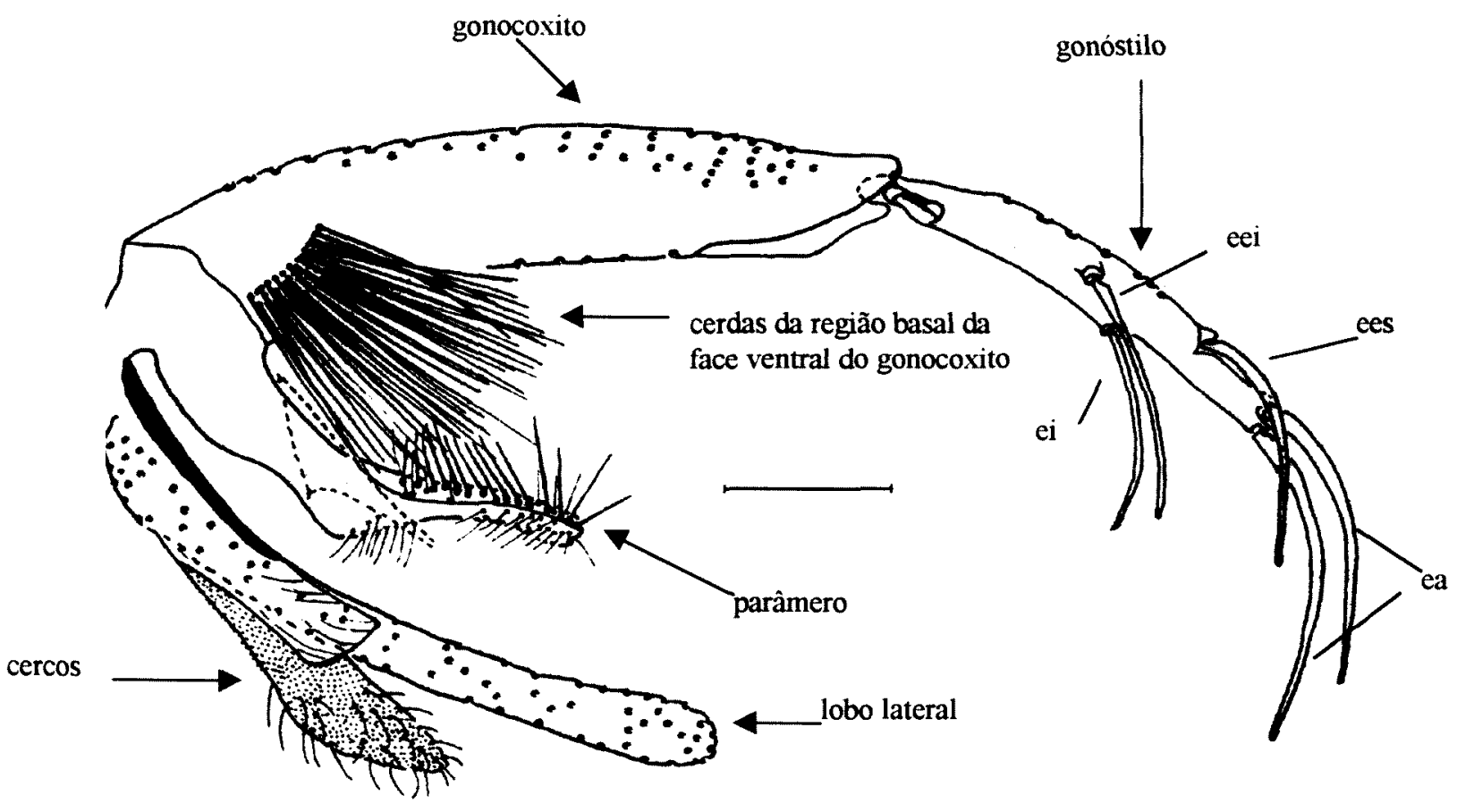

Figura 28 - Genitália de Lutzomyia kirigetiensis: ea = espinhos apicais do gonóstilo; ei = espinho interno; eei = espinho interno inferior; ees = espinho externo superior 


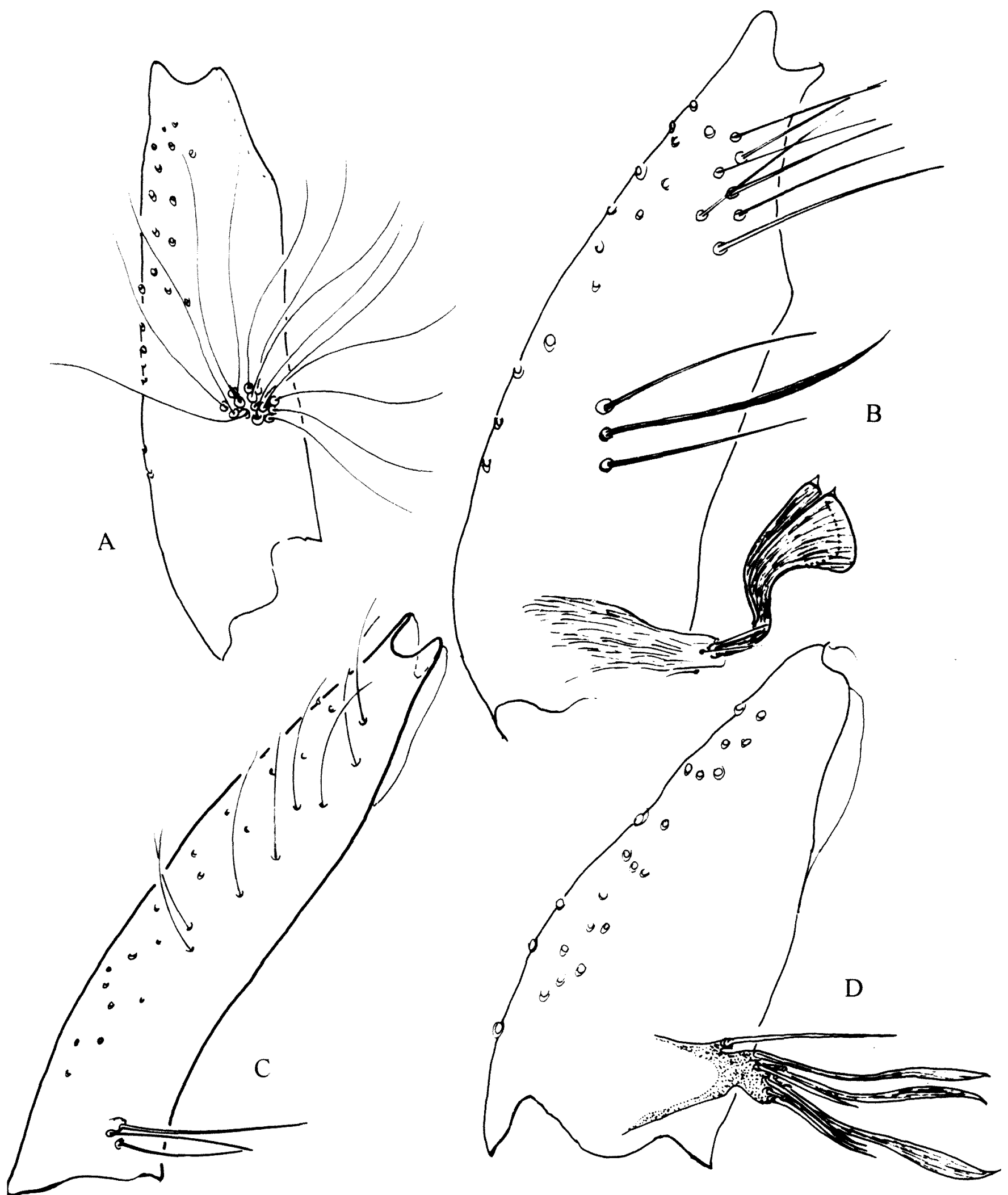

Figura 29 - Aspecto e forma de implantação das cerdas da região basal da face ventral do gonocoxito de espécies de Lutzomyia: $\mathrm{A}=$ L. cruciata com cerdas finas implantadas em capitulo; $\mathrm{B}=L$. almerioi com cerdas foliáceas implantadas em tubérculo e com presença de cerdas bem desenvolvida (s) no terço mediano da face ventral; $\mathrm{C}=L$. hartmanni com cerdas finas impalntadas isoladamente; $\mathrm{D}=L$. carvalhoi com cerdas smifoliáceas implantadas em tubérculo.

Aneros A-76 

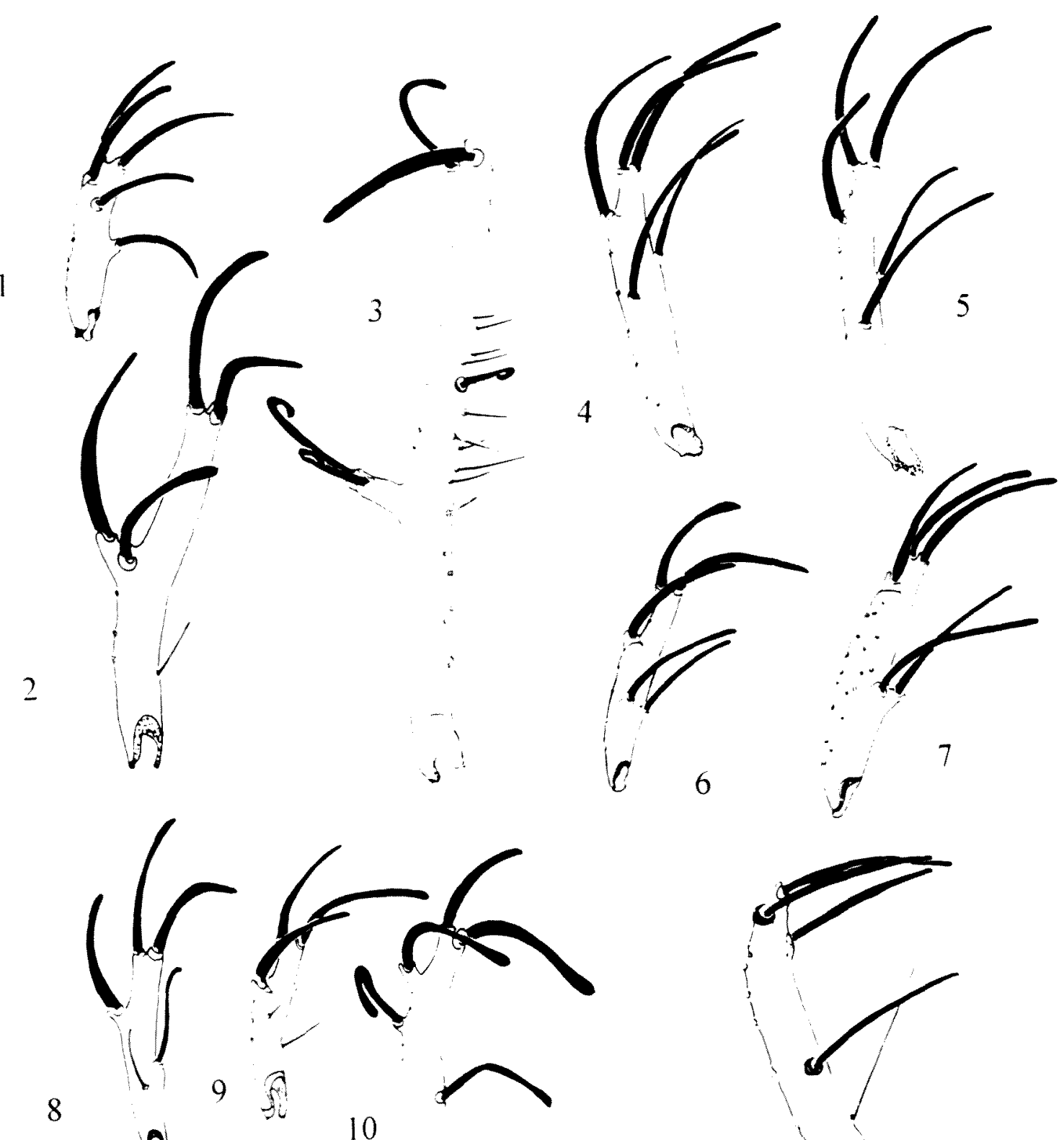

$\Omega$

10
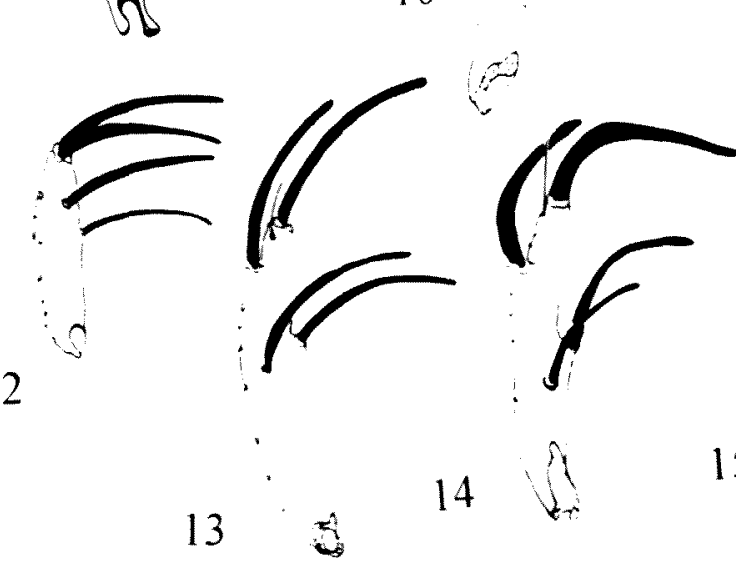

15
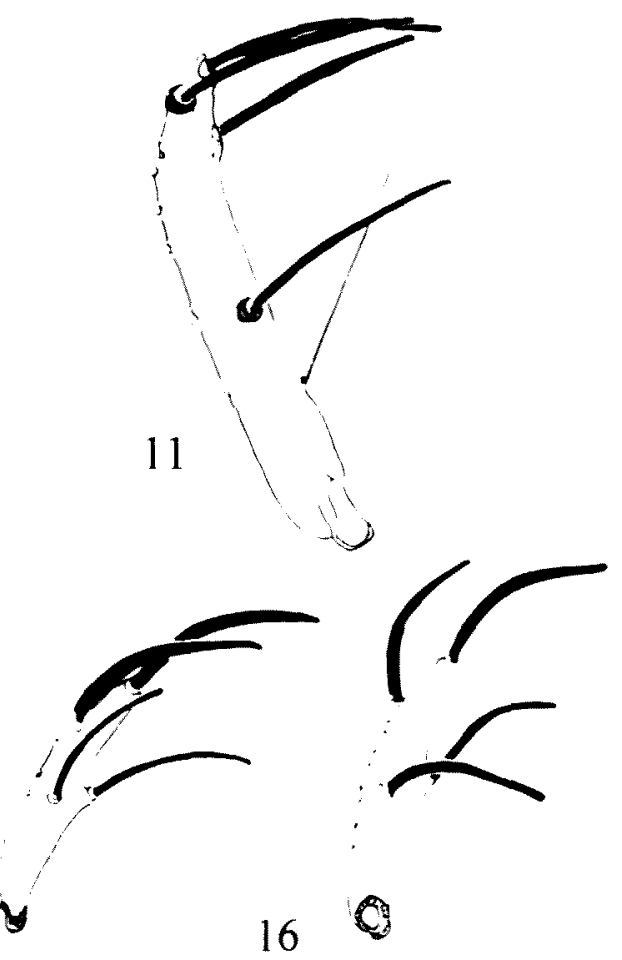

Figura 30 - Gonóstilo de espécies de Phlebotominae: $1=$ E. piauiensis; $2=O$. toroensis; $3=B$. nitzulescui; $4=L$. pescei, $5=L$. tejadai, $6=L$. noguchii; $7=L$. peruensis; $8=L$. bicornuta; $9=D$. samueli; $10=M$. vexator $; 11=W$. euniceae $; 12=L$. ischnacantha; $13=L$. castroi $; 14=L$. longipalpis, $15=$ L. dispar $; 16=$ L. cruciata . 


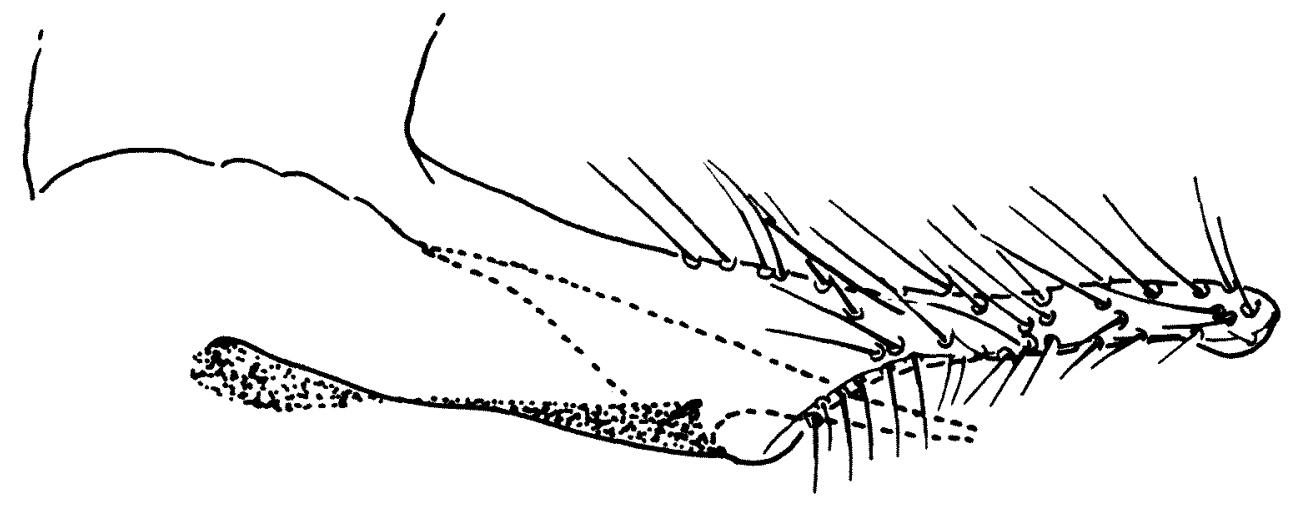

A

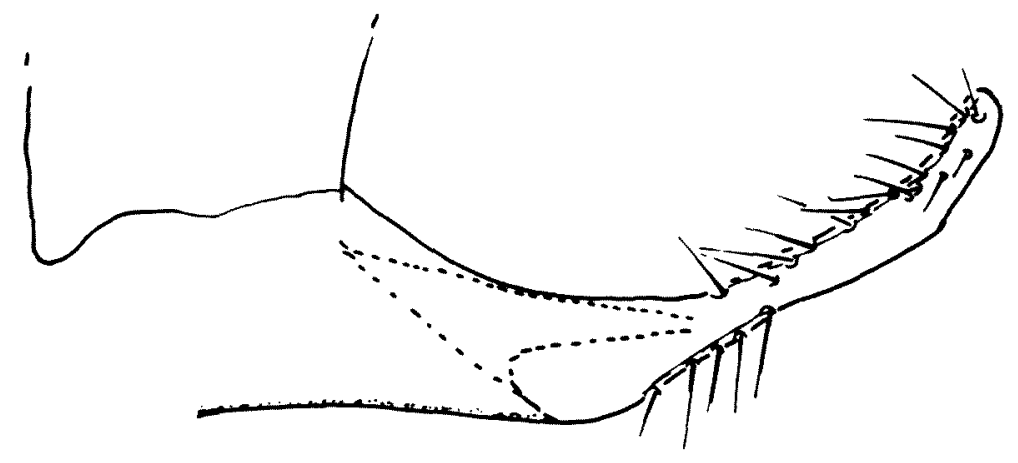

B

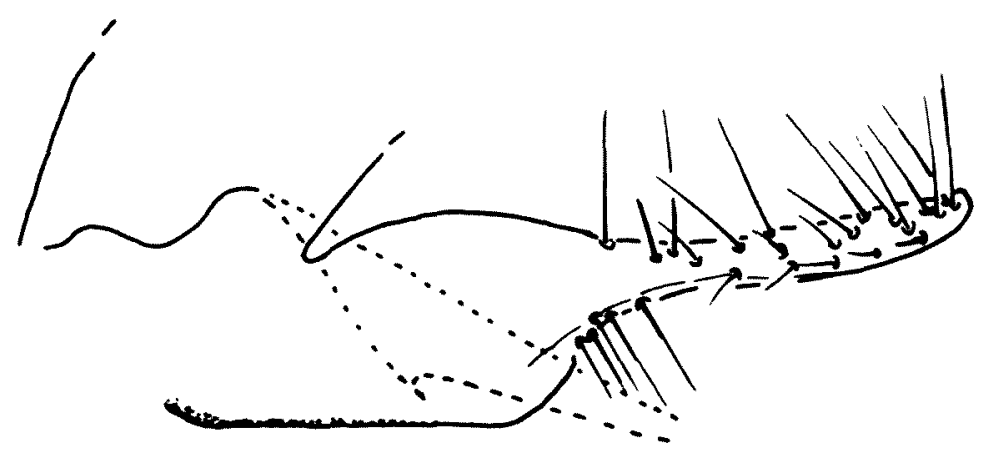

C

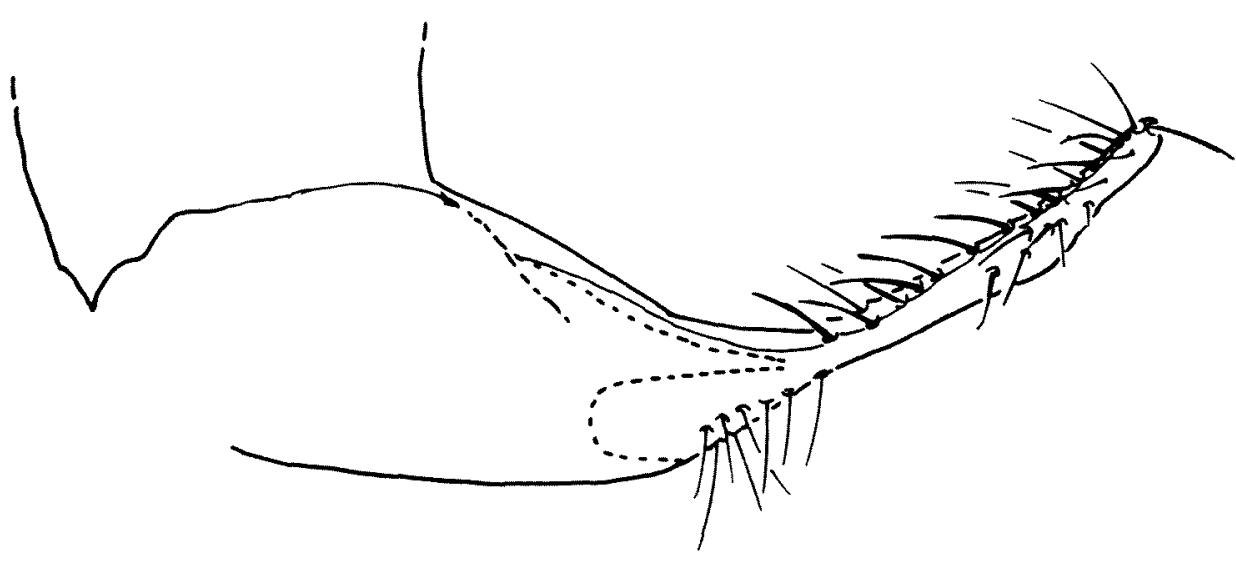

D

Figura 31 - Parâmeros de espécies de Lutzomyia. $\mathrm{A}=$ L. guderiani; $\mathrm{B}=L$. rispaili $; \mathrm{C}=L$. cruciata; $\mathrm{D}=$ L. munaypata 


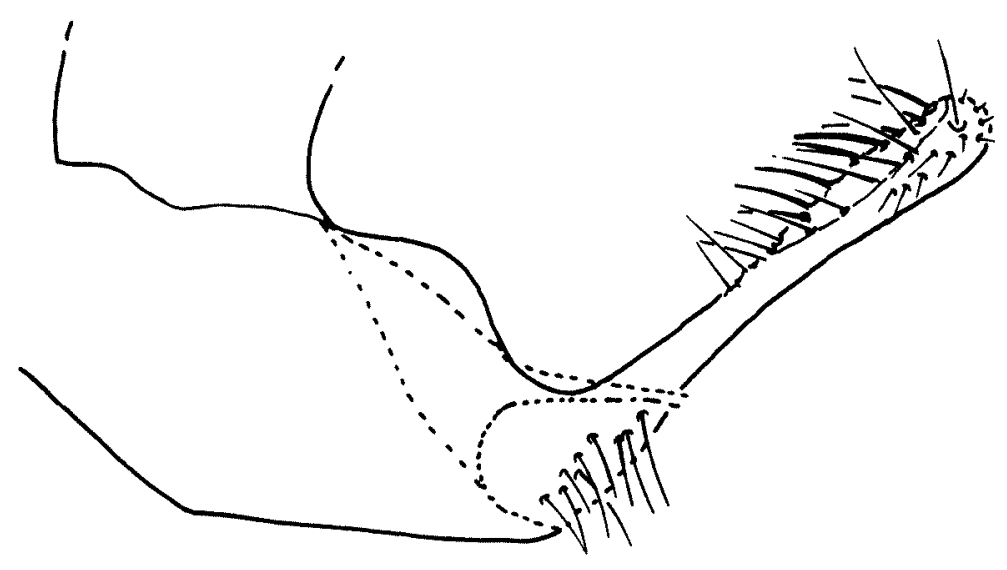

E

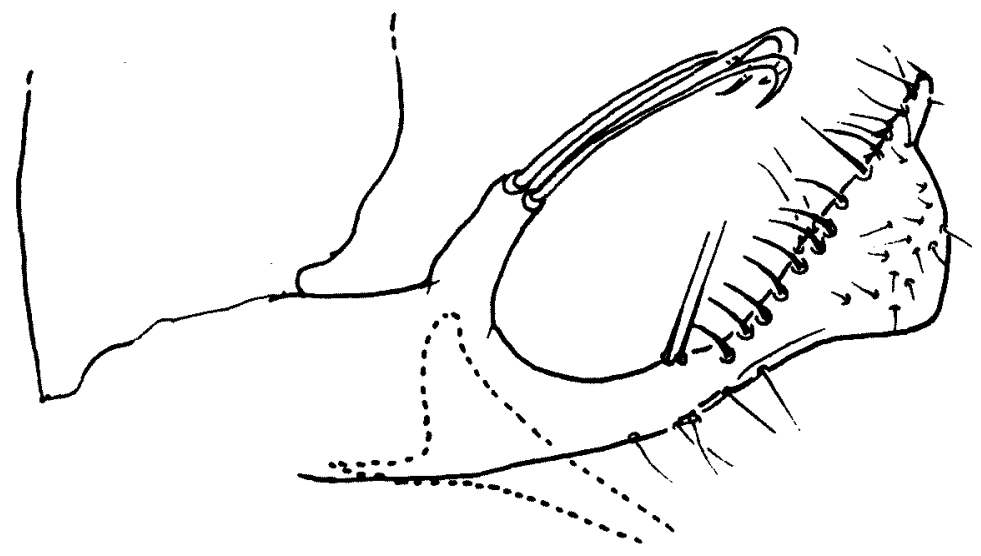

F

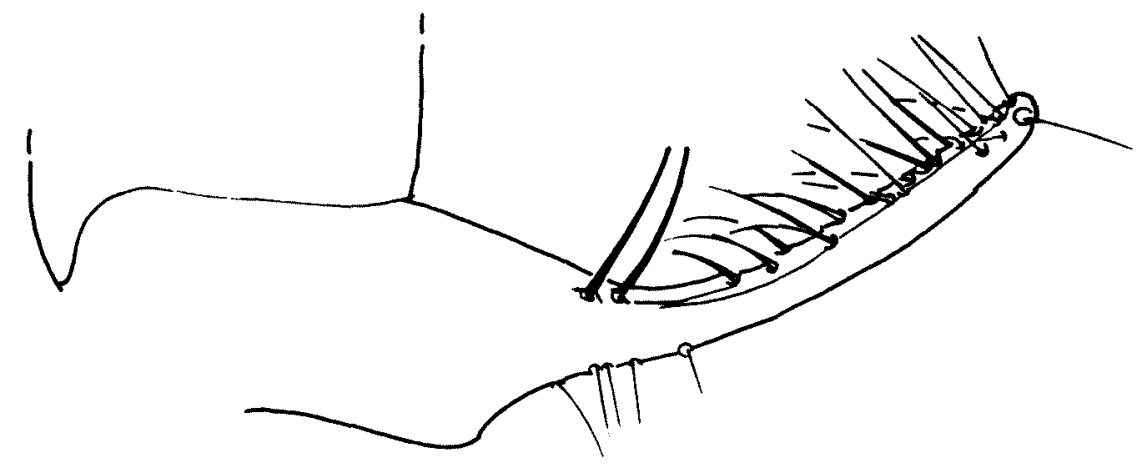

G

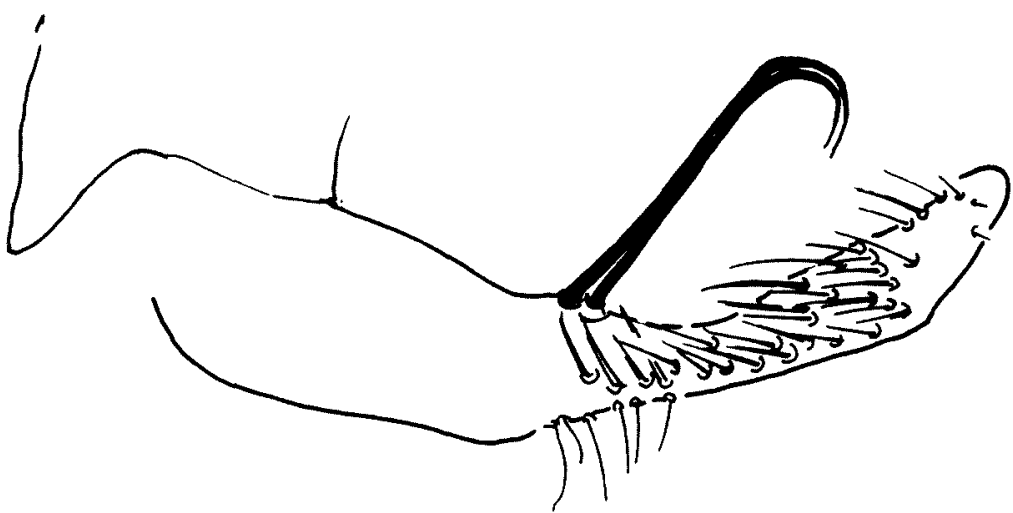

$\mathrm{H}$

Figura 32 - Parâmeros de espécies de Lutzomyia $. \mathrm{E}=$ L. quillabamba; $\mathrm{F}=$ L. dispar; $\mathrm{G}=$ L.ischnacantha $\mathrm{H}=L$.renei 

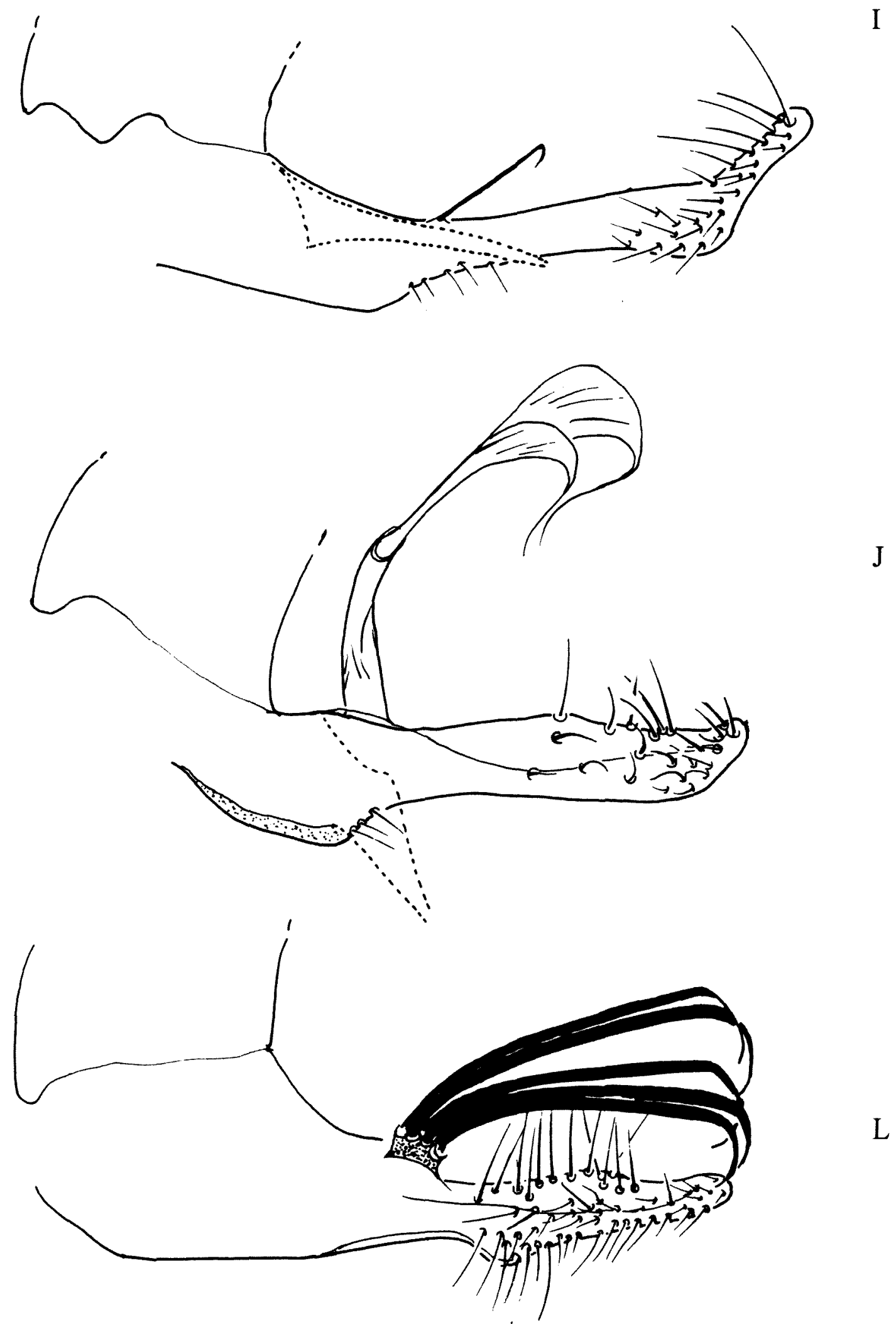

L

Figura 33 - Parâmeros de espécies de Lutzomyia. $\mathrm{I}=L$. castroi $; \mathrm{J}=$ L. lichyi $; \mathrm{L}=L$. battistinii

Anexos A-80 


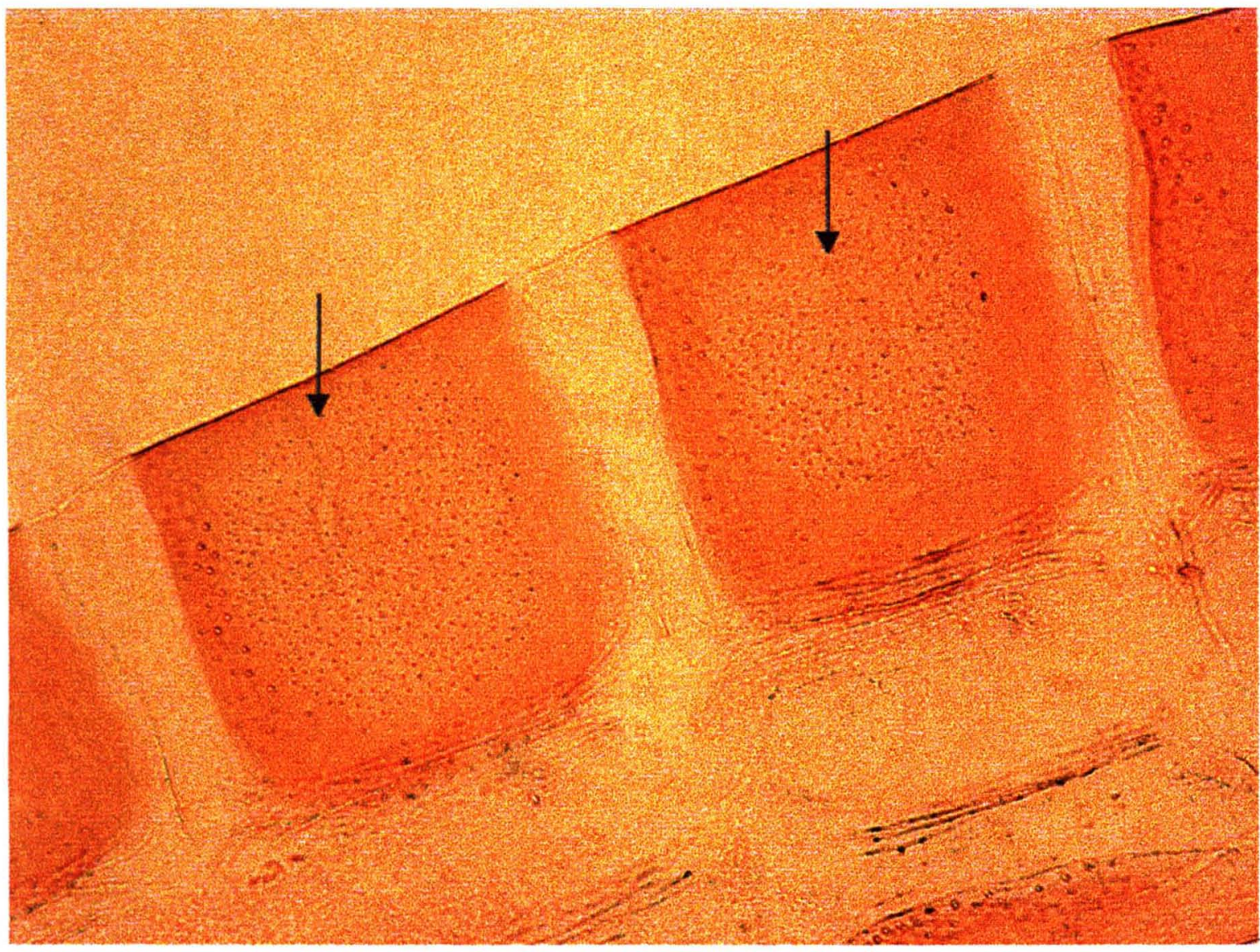

Figura 34 - Papila tergais no terceiro e quarto tergito abdominais de macho de L. longipalpis 

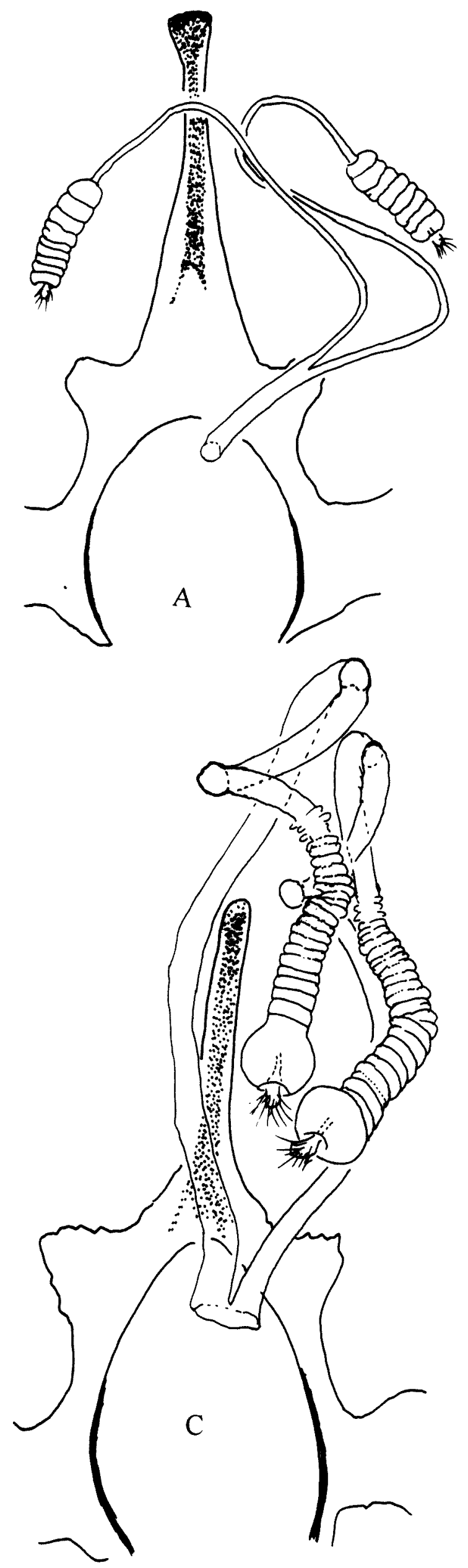
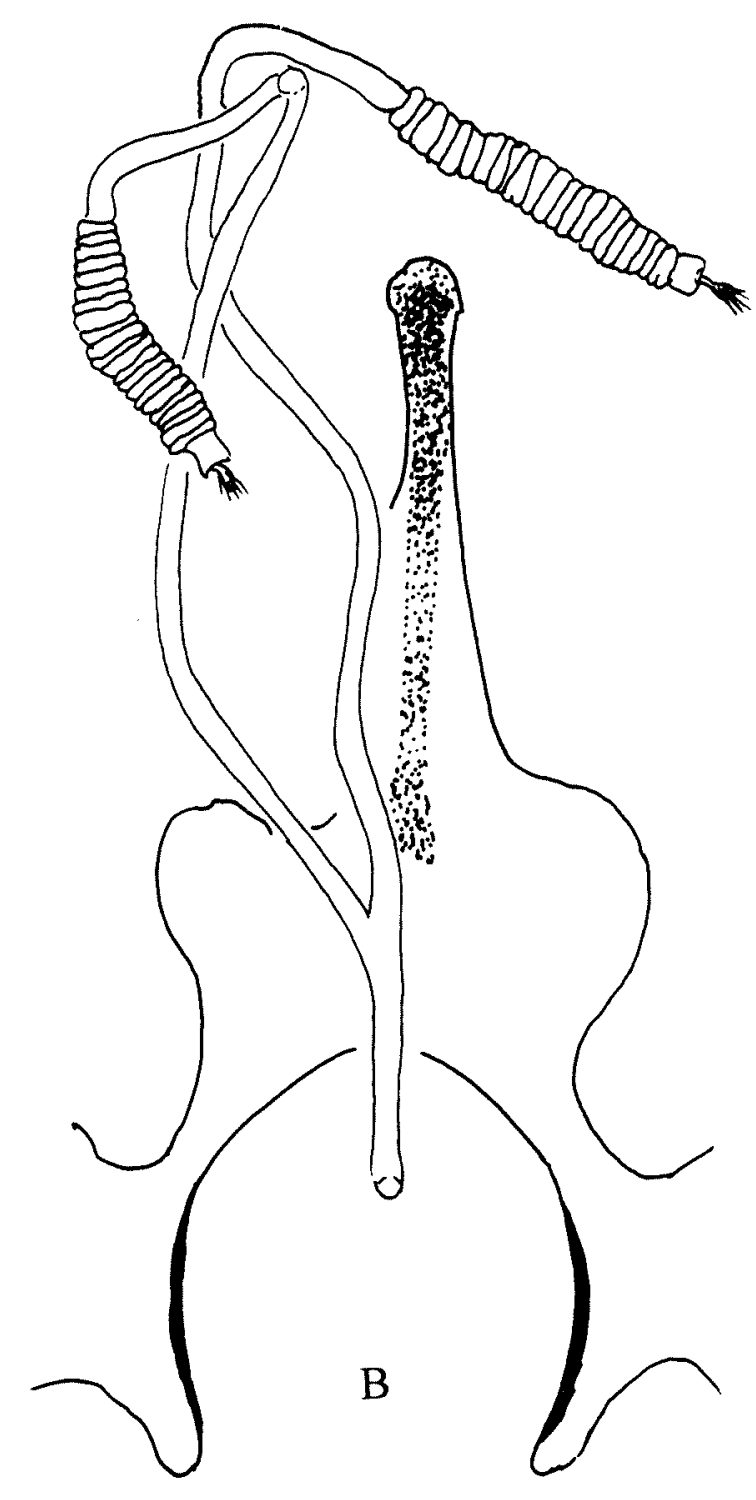

Figura 35 - Espermatecas de Lutzomvia. $\mathrm{A}=$ L. longipalpis; $\mathrm{B}=$ L. munypata $; \mathrm{C}=L$. sherlocki 